\title{
Characterization of $\mathrm{NfsB}$,
}

the minor oxygen-insensitive nitroreductase of Escherichia coli.

\author{
by \\ Jacqueline A. Whiteway

\begin{abstract}
A thesis submitted to
the Faculty of Graduate Studies and Research

in partial fulfillment of

the requirements for the degree of
\end{abstract}

Doctor of Philosophy
College of Natural Sciences
Carleton University
Ottawa, Ontario

(C2002 Jacqueline A. Whiteway 
National Library

of Canada

Acquisitions and

Bibliographic Services

395 Wellington Street

Ottawa ON K1A ON4

Canada
Bibliothèque nationale

du Canada

Acquisitions et services bibliographiques

395, rue Wellington

Ottawa ON K1A ON4

Canada
Your tie Votre retirence

Our the Notre rifirience
The author has granted a nonNational Library of Canada to reproduce, loan, distribute or sell copies of this thesis in microform, exclusive licence allowing the paper or electronic formats.

L'auteur a accordé une licence non exclusive permettant à la Bibliothèque nationale du Canada de reproduire, prêter, distribuer ou vendre des copies de cette thèse sous la forme de microfiche/film, de reproduction sur papier ou sur format électronique.

The author retains ownership of the copyright in this thesis. Neither the thesis nor substantial extracts from it may be printed or otherwise reproduced without the author's permission.
L'auteur conserve la propriété du droit d'auteur qui protège cette thèse. $\mathrm{Ni}$ la thèse ni des extraits substantiels de celle-ci ne doivent être imprimés ou autrement reproduits sans son autorisation. 
The undersigned hereby recommend to

the Faculty of Graduate Studies and Research

acceptance of the thesis

Characterization of $\mathrm{NfsB}$,

the minor oxygen-insensitive nitroreductase of Escherichia coli

Submitted by

Jacqueline A. Whiteway, B.Sc.

in partial fulfilment of the requirements

for the degree of Doctor of Philosophy

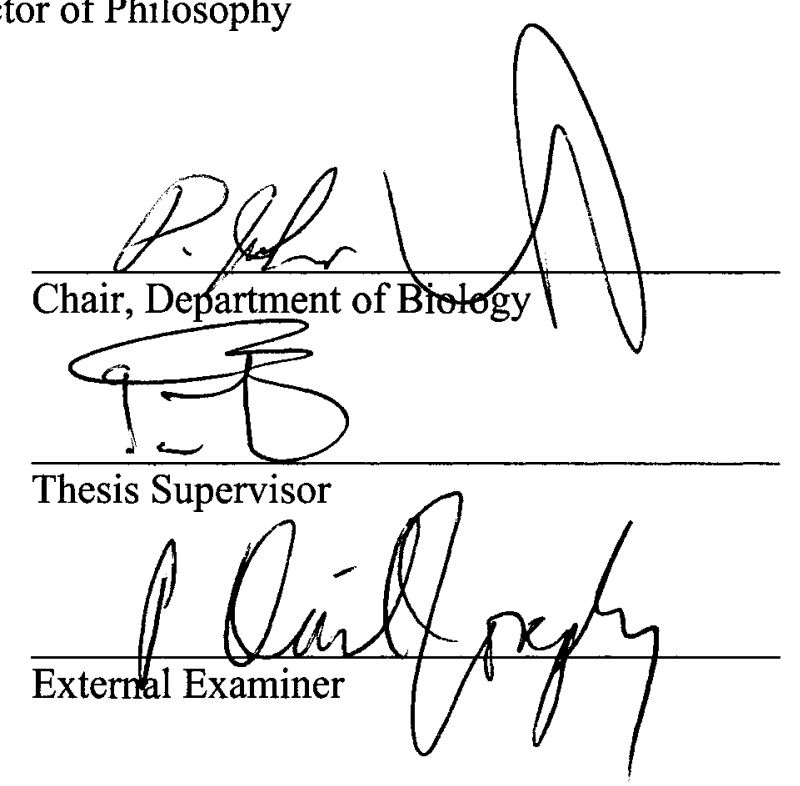

Carleton University

August 29, 2002

ii 


\begin{abstract}
Nitro-substituted compounds constitute a wide range of chemicals whose potent biological activity has significant human health implications. The biological activity of nitro-substituted compounds is derived from reductive metabolism of the nitro moiety, a process catalyzed by a variety of nitroreductase (NR) activities that can be classified on the basis of oxygen sensitivity. The recent accumulation of genomic data has helped lead to the recognition of the oxygen-insensitive NRs as a widespread family of enzymes that now includes an estimated 140 sequences in organisms throughout the three main domains of life. The physiological role of these enzymes remains a mystery. Escherichia coli has two oxygen-insensitive NRs, NfsA and NfsB. NfsB, the focus of this study, is a FMN-containing flavoprotein with a monomeric molecular mass of approximately 24 $\mathrm{kDa}$, and can use either NADH or NADPH as a source of reducing equivalents.
\end{abstract}

The research reported in this thesis contributes to the characterization of $E$. coli NfsB in four main ways. First, in a combined effort to elucidate the development of 5nitrofuran resistance in $E$. coli, it was determined that the increased resistance associated with second step nitrofuran-resistant mutants is a consequence of mutational inactivation of the $n f_{s} B$ gene. An unusually high proportion of mutational events in $n f_{s} B$ involved IS elements. Second, it was demonstrated that NfsB is expressed in response to induction of the SoxRS and MarRAB regulons, key components of the $E$. coli global response to environmental stress. Third, an affinity purification scheme for $\mathrm{NfsB}$ was developed and the protein was kinetically characterized. Detailed kinetic analysis using nitrofurazone as a substrate shows that $\mathrm{NfsB}$ has a catalytic specificity $\left(\mathrm{k}_{\mathrm{cat}} / \mathrm{Km}\right.$ value) of approximately $200 \mu \mathrm{M}^{-1} \mathrm{sec}^{-1}$ for NADH and NADPH. Mechanistically, the kinetic data are consistent 
with the notion that $\mathrm{NfsB}$ uses a ping-pong bi-bi mechanism. Seventeen individual mutations were introduced by PCR site-directed mutagenesis. One mutation, G158S, resulted in a decreased specificity for NADPH, while another, F70S, increased the turnover number $\left(\mathrm{k}_{\mathrm{cat}}\right)$ for nitrofurazone without affecting cofactor specificity. Fourth, the active site of $\mathrm{NfsB}$ was characterized by computer analysis, leading to suggestions for protein pockets that could be targets for drug development. 


\section{ACKNOWLEDGMENTS}

Dr. Iain B. Lambert, for invaluable advice, support (intellectual, emotional, financial), good parties and lots of editing. Dr. Suzanne Paterson, for being an endless source of ideas and technical advice, as well as a good friend and mentor in many ways, especially in the rewards of taking shortcuts. Dr. R. Campbell Wyndham, Dr. Guy Drouin, Dr. James Cheetham, Dr. Myron Smith, Dr. Ken Storey and Dr. Steve Brooks for their generous contributions of advice, guidance and the use of equipment. (Steve receives my gratitude despite his counsel that led me to reject a whole summer's worth of work. Besides, he also made it possible to do five times the work in half the time.)

My colleagues and friends, for their help and kinship during my graduate years. I can't list everyone here, so I will only mention Cristina Micali, Miguel Providenti, Katalin Bertenyi, Reza Nokhbeh, Sheryl Hubbard, Peter Koziarz, Craig Carroll, Dustin Hittel, and Fiona Chambers, all of whom directly influenced the outcome of this work. Allison Hunter, for a friendship without which I probably would've imploded. Stephen Giguere, for preventing some major catastrophes.

Many undergraduate students, for being some of my best teachers: John Veall, Brian Julian and Todd Hodgson to name a few. Their questions and ideas were constant reminders that I will never know it all.

Brian and the rest of my family, for the astonishing amount of support and free babysitting services that were essential for the completion of this thesis. Carmen: without you I would probably still be in the lab tying up some experiments.

Thank-you 


\section{TABLE OF CONTENTS}

Title Page......................................................................................................................................................

Acceptance form .......................................................................................................................................ii

Abstract ................................................................................................................................................iii

Acknowledgments................................................................................................................................. v

Table of Contents ............................................................................................................................................. vi

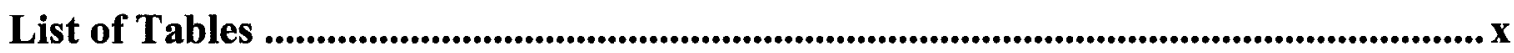

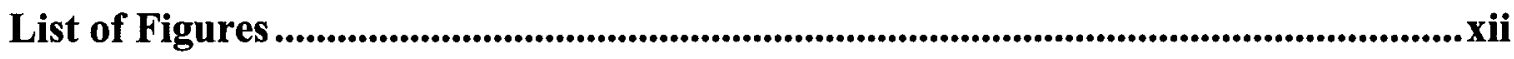

List of Abbreviations....................................................................................................................................... Xv

Chapter One: General Introduction................................................................................................. 1

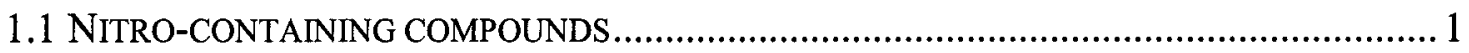

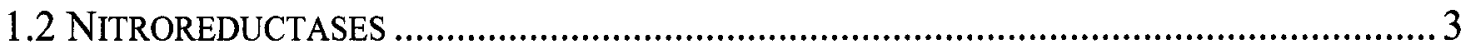

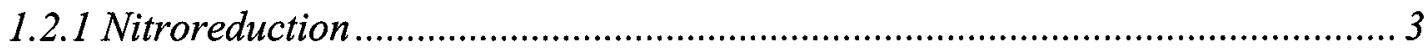

1.2.2 Characterization of nitroreductases ……………......................................... 6

1.2.2.1 Characteristics of the NR family proteins ................................................ 13

1.2.2.2 The NR family can be divided into two subgroups................................... 14

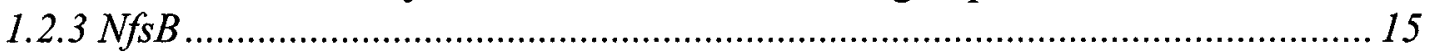

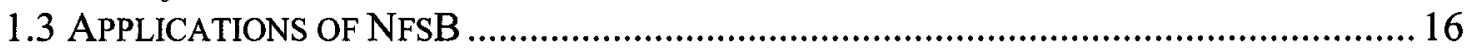

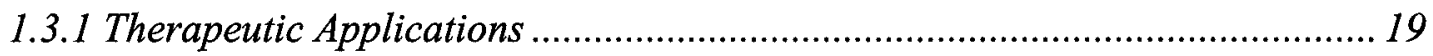

1.3.1.1 Antimicrobial mechanisms and drug development..................................... 19

1.3.1.2 Cancer Therapy .................................................................................... 19

1.3.2 Detection and analysis of nitro-containing compounds....................................2 21

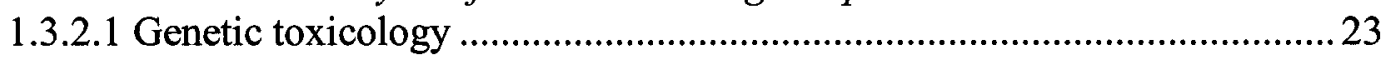

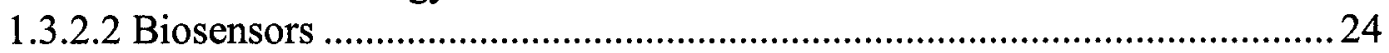

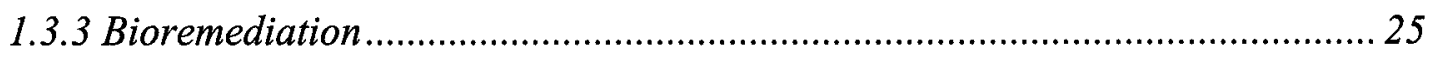

1.4 RATIONALE AND RESEARCH OBJECTIVES..............................................................2

1.4.1 How does resistance to 5-nitrofurans develop in E. coli? ...............................2 26

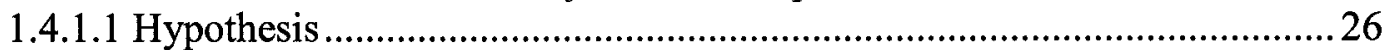

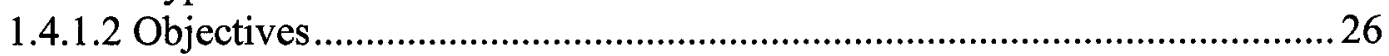

1.4.2 Is NfsB part of the Sox/Mar response to environmental stress? ...................... 27

1.4.2.1 Hypothesis .................................................................................................2

1.4.2.2 Objectives ................................................................................................ 27

1.4.3 What are the determinants of cofactor specificity in $N f S B$ ? ............................ 28

1.4.3.1 Hypothesis ........................................................................................... 28

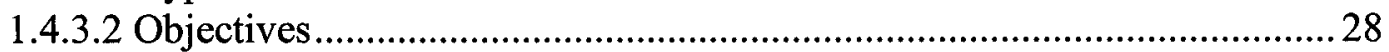

1.4.4 What can computer analysis reveal about the active site of $N f S B$ ?.................... 29

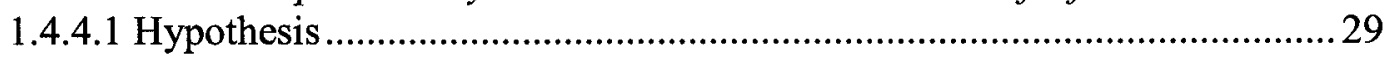




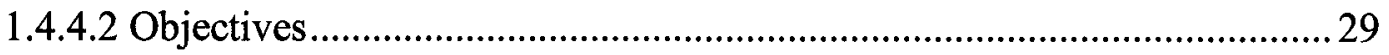

Chapter Two: The development of 5-nitrofuran resistance in $E$. coli..........................30

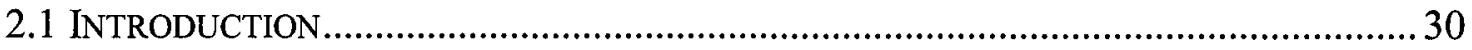

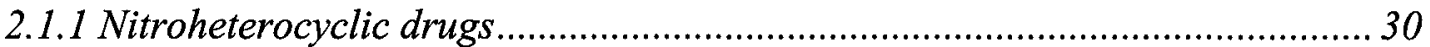

2.1.2 Metabolic activation of nitroheterocyclic drugs ............................................. 31

2.1.3 Microbial resistance to 5-nitrofurans .............................................................. 32

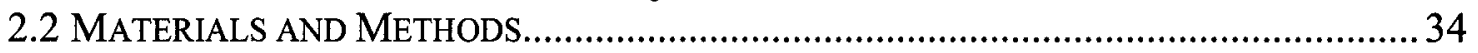

2.2.1 Bacterial strains, plasmids and growth conditions.......................................... 34

2.2.2 Isolation of furazolidone resistant strains.......................................................... 35

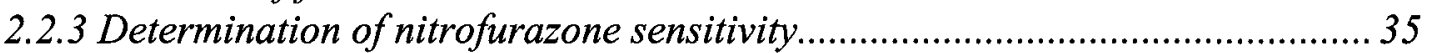

2.2.4 Preparation of clarified cellular extracts........................................................ 35

2.2.5 Determination of protein concentration........................................................ 36

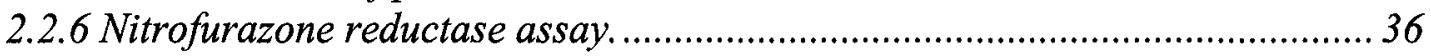

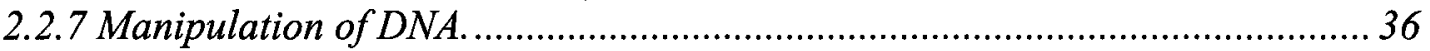

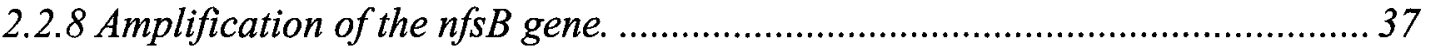

2.2.9 Cloning of $n f_{s} B$.

2.2.10 Sequencing of clones and PCR products................................................... 41

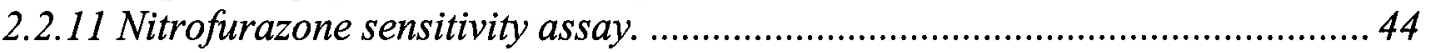

2.2.12 Nitrofurazone and furazolidone susceptibility assay......................................4 44

2.2.13 Chloramphenicol acetyltransferase assay. ...................................................45

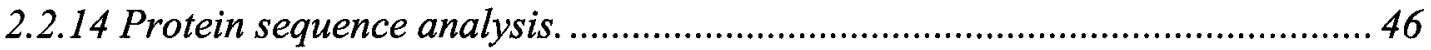

2.2.15 Induction of nitroreductase by paraquat and salicylate. ................................ 46

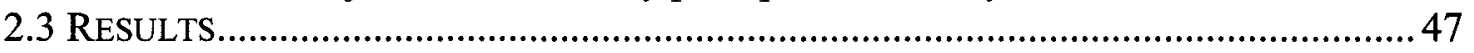

2.3.1 Isolation of mutant strains. .......................................................................... 47

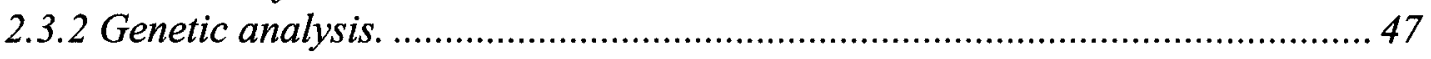

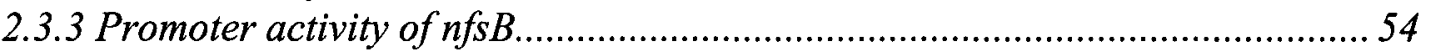

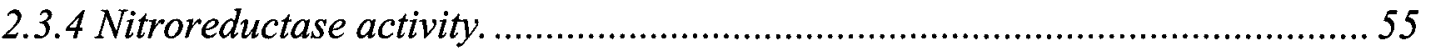

2.3.5 Restoration of nitrofuran sensitivity by cloned $N f S A$ and $N f S B \ldots \ldots \ldots \ldots \ldots \ldots \ldots . . .56$

2.3.6 EDTA treatment and nitrofuran susceptibility ............................................... 58

2.3.7 Induction of nitroreductase by paraquat and salicylate ..................................5 58

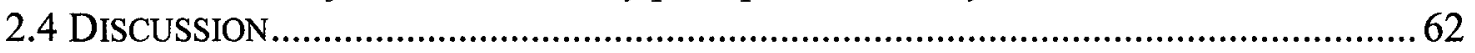

2.4.1 Isolation of nitrofuran resistant strains ........................................................... 62

2.4.2 Characterization of the nitrofuran resistant mutants........................................63 63

2.4.3 IS element hotspots are likely present in the $n f s B$ gene ...................................66

2.4.4 Implications of the mutations for the NfsB enzyme...........................................69 69

2.4.5 NfsB: a putative member of the SoxRS/MarRAB stress response ..................... 74

Chapter Three: The kinetic characterization of $E$. coli NfsB .......................................80

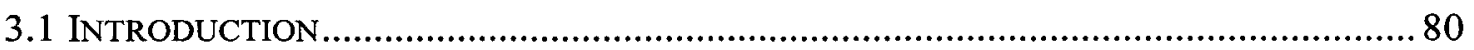

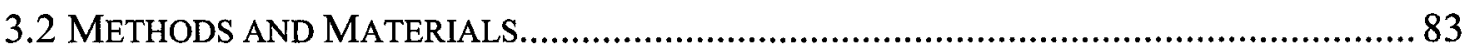

3.2.1 Bacterial strains, plasmids and growth conditions ........................................ 83

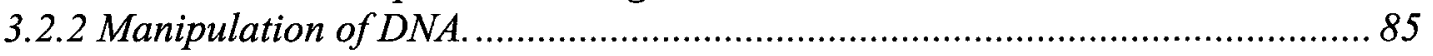

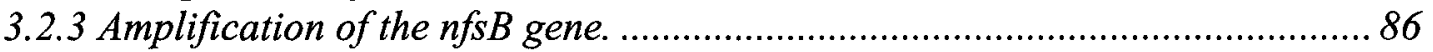

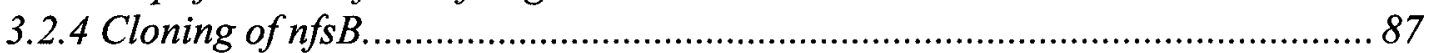




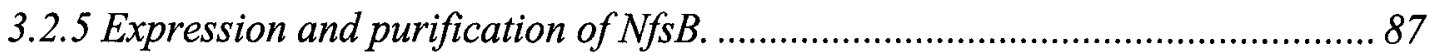

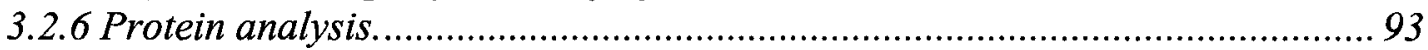

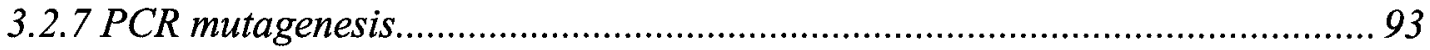

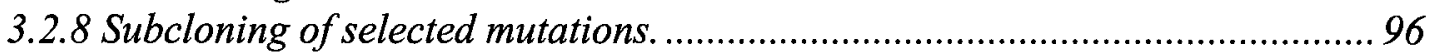

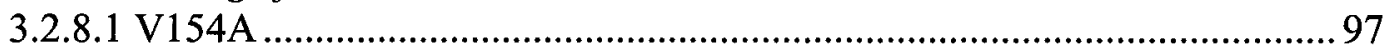

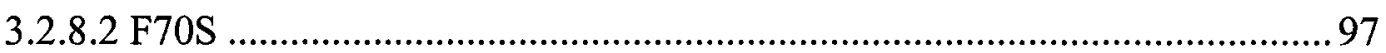

3.2.9 Confirmation of protein expression and nitroreductase activity ..................... 98

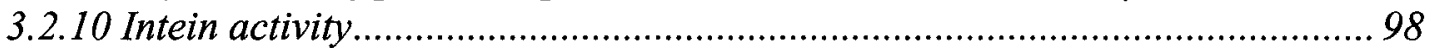

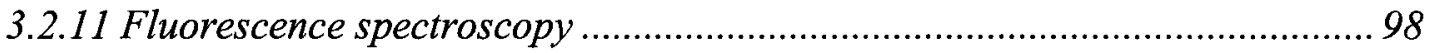

3.2.12 Ultraviolet spectroscopy ......................................................................... 99

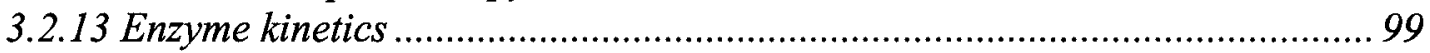

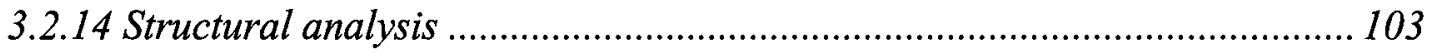

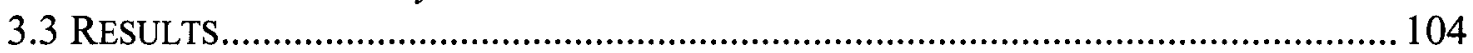

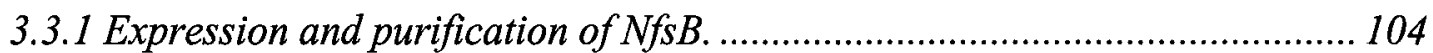

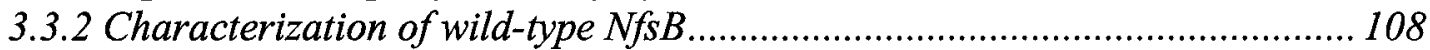

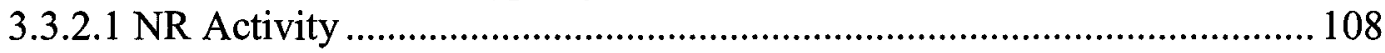

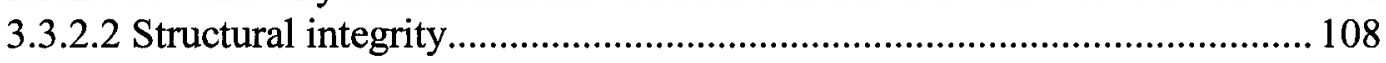

3.3.2.3 Enzyme kinetics ............................................................................... 111

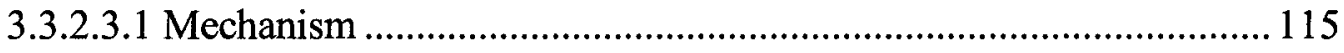

3.3.2.3.2 Kinetic parameters...................................................................... 115

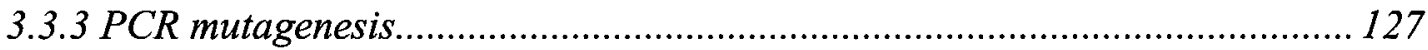

3.3.4 Purification and characterization of mutants .............................................. 133

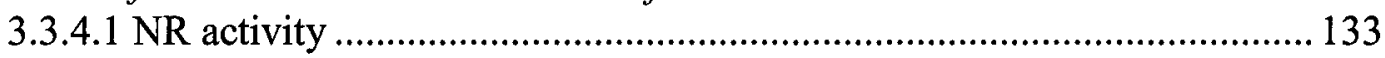

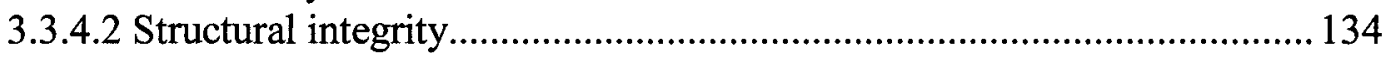

3.3.4.3 Enzyme kinetics ............................................................................... 137

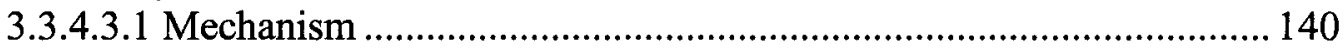

3.3.4.3.1.2 Substrate inhibition ................................................................ 140

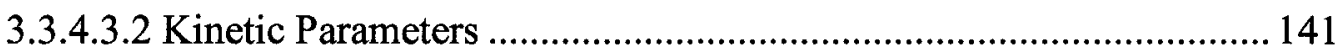

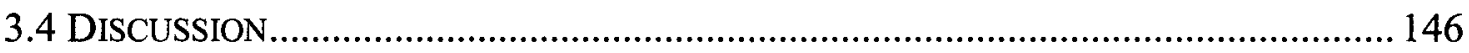

3.4.1 Wild-type characterization ......................................................................... 146

3.4.1.1 Expression and purification of NfsB ..................................................... 146

3.4.1.2 Structural integrity......................................................................... 146

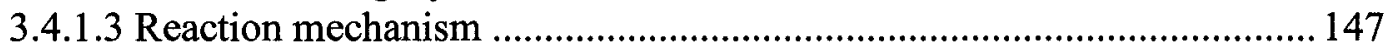

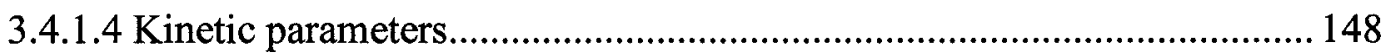

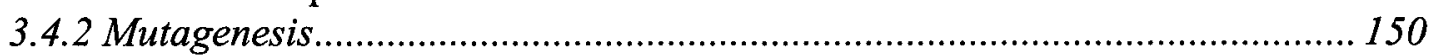

3.4.3 Residues in target region may influence NADPH utilization.......................... 152

3.4.4 F70S has no effect on cofactor specificity..................................................... 159

Chapter Four: Computer analysis of the NfsB active site................................................161

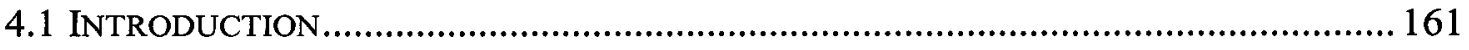

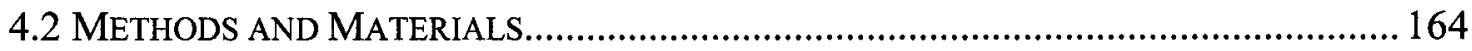

4.2.1 Identification and visualization of $N f S B$ homologues................................... 164

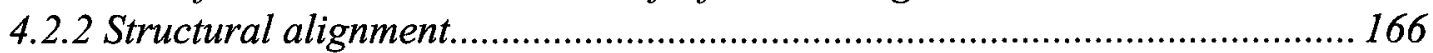

4.2.3 Determination of interatomic contacts................................................... 166

4.2.4 Identification of protein pockets and cavities .......................................... 167 


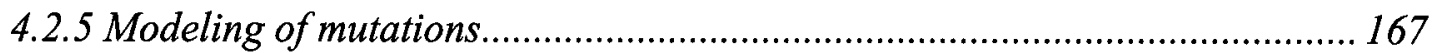

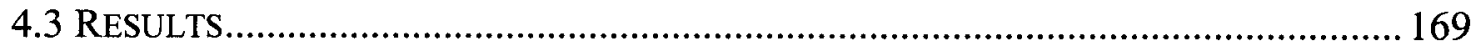

4.3.1 Structural alignment of $N f_{s} B$ and its homologues................................... 169

4.3.2 Nfs B: Protein pockets and structural contacts.......................................... 169

4.3.2.1 The major pocket: FMN, NAD(P)H and substrate binding ..................... 174

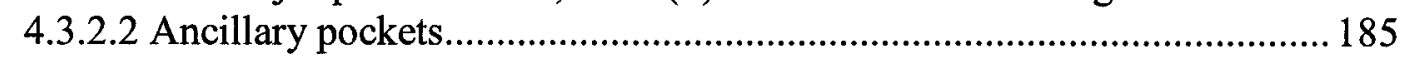

4.3.2.2.1 Conserved residues.................................................................... 186

4.3.2.2.2 Conformational changes in NIO-bound NfsB............................... 187

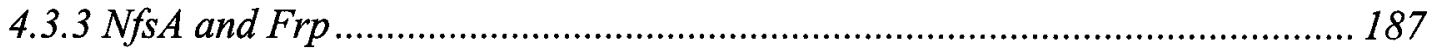

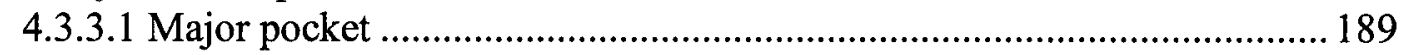

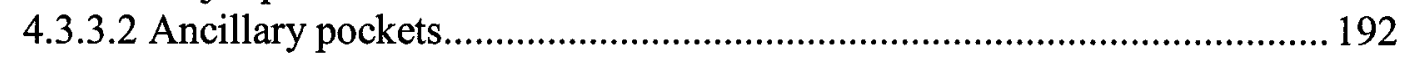

4.3.4 Analysis of the mutant structures .......................................................... 192

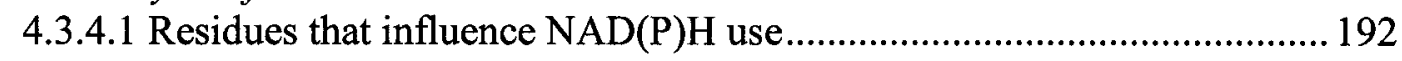

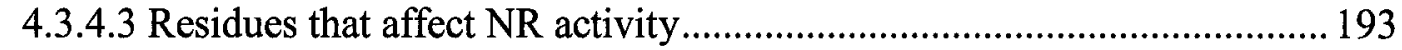

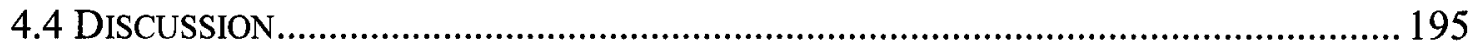

4.4.1 Characterization of the oxidized $N f S B$ active site .................................... 195

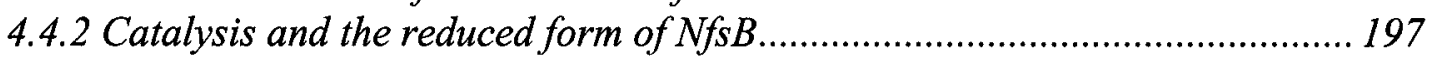

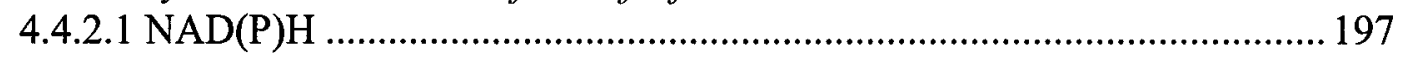

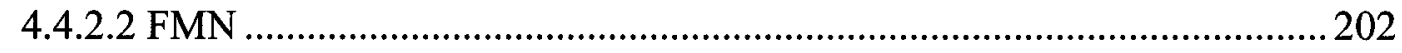

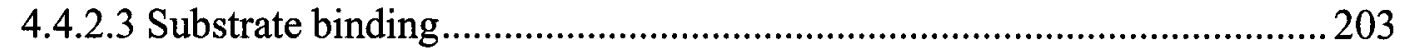

4.4.3 NfSA and NfsB: A revealing comparison...................................................206

4.4.4 The implications for structure-based drug development..........................210

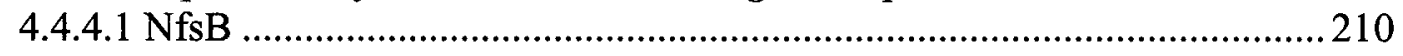

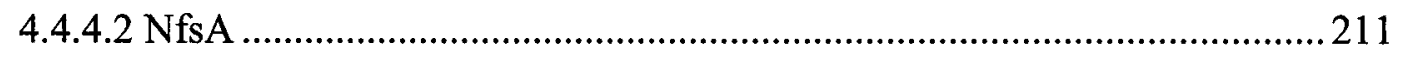

Chapter Five: Conclusions and suggestions for further research ...........................213

5.1 DEVELOPMENT OF 5-NITROFURAN RESISTANCE IN E. COLI .................................213

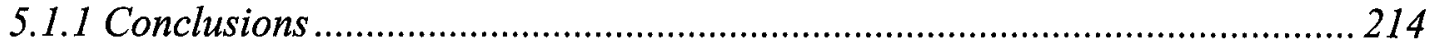

5.1.2 Suggestions for further research ........................................................ 214

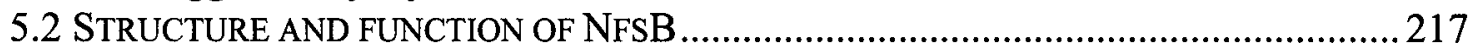

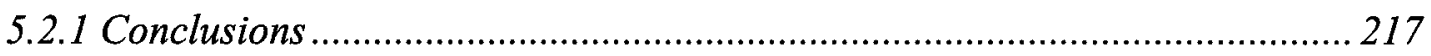

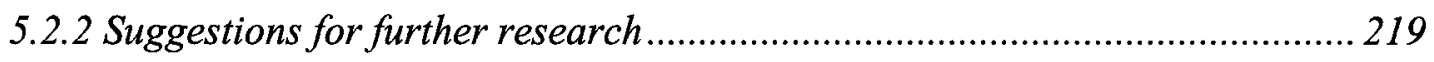

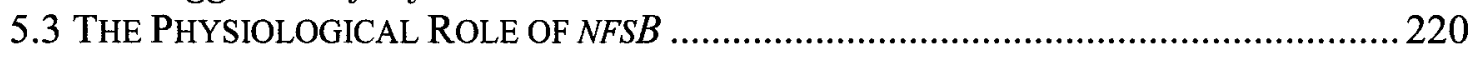

5.3 .1 Suggestions for further research .................................................... 221

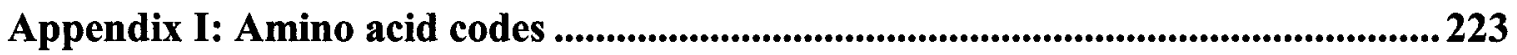

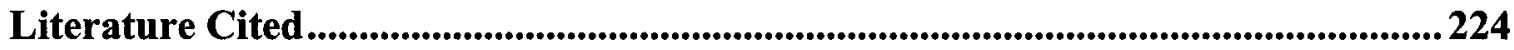




\section{LIST OF TABLES}

Table

1.1 Taxonomic distribution of oxygen-insensitive nitroreductases.

9

1.2 Studies of gene directed enzyme prodrug therapy (GDEPT) that use the 22 $\mathrm{NfsB} / \mathrm{CB} 1954$ enzyme/prodrug pair.

2.1 Primers used in this study. $\quad 40$

2.2 Mutations in the $n f_{s} A$ and $n f_{s} B$ genes of first- and second-step nitrofuran 49 resistant mutants, and the nitrofurazone reductase activities of the mutant cellular extracts.

2.3 Mutation distribution in $n f s A$ and $n f s B$ of nitrofuran resistant mutants.

2.4 Diameters ( $\mathrm{mm}$ ) of cleared zones in a lawn of each strain with and without vector encoded NfsA (pTAA) and NfsB (pTAB) after the application of $5 \mathrm{mg} / \mathrm{ml}$ nitrofurazone.

2.5 Sequence identities of putative NfsB homologues and paralogues. Percent identity of the NR fingerprint region (Ser-37 to Val-50) is included.

3.1 The primers used in this study.

3.2 Nitrofurazone reductase specific activities ( $\mathrm{nmol} / \mathrm{min} / \mathrm{mg}$ ) of cell extracts containing vector encoded wild-type and mutated NfsB.

3.3 Fluorescence emission peaks (excitation at $290 \mathrm{~nm}$ and $464 \mathrm{~nm}$ ) and UV 113 absorbance values $(280 \mathrm{~nm})$ for wild-type and mutant $\mathrm{NfsB}$ proteins.

3.4 $\mathrm{Km}(\mu \mathrm{M})$ and $\mathrm{k}_{\mathrm{cat}}\left(\mathrm{sec}^{-1}\right)$ values of wild-type and mutant NfsB proteins with NADH and NADPH as cofactors and nitrofurazone (NF) as the electron acceptor.

3.5 Mutations introduced by targeted PCR mutagenesis. The target region includes residues Gly-153 to Leu-159 in NfsB.

3.6 Predicted frequency of mutation number in the target region of $\mathrm{NfsB}$, and the actual frequency in 98 mutants. 
3.7 Ki values $(\mu \mathrm{M})$ for nitrofurazone with each cofactor in wild-type NfsB and two of the mutant enzymes.

3.8 Evolutionarily accepted amino acid substitutions based upon the mutation data matrix compiled by Dayhoff et al., (1983).

4.1 The proteins analyzed in this study. All structures were resolved by XRay crystallography.

4.2 NfsB mutations, and one NfsA mutation, that were modeled in this study.

4.3 Volume of the major pocket (I) and other pockets referred to in this study. The number of solvent accessible mouth openings to each pocket is indicated in parentheses.

4.4 Amino acid composition of pockets I-IV in NfsB and NfsB complexed with NIO. 


\section{LIST OF FIGURES}

Figure

1.1 Proposed mechanism for the metabolic activation of nitro-substituted compounds.

1.2 Alignment of characterized NfsB-like nitroreductases.

2.1 Nucleotide sequence of the PCR-generated fragment containing the $n f s B$ gene from $E$. coli $\mathrm{AB} 1157$.

2.2 The linear $\mathrm{pCR}{ }^{\circledR} 2.1$ vector (Invitrogen) is shown in the centre and the clones pTAA and pTAB containing PCR amplified $n f s A$ and $n f s B$, respectively, are indicated below.

2.3 Plasmid clones of $n f s B$. (a) pJAB: PCR-amplified $n f s B$ inserted in pUC118. (b) pJAC: Truncated $n f s B$, lacking the putative transcription terminator, inserted into the pKK232-2 vector containing the CAT gene.

2.4 The inducibility of $\mathrm{NAD}(\mathrm{P}) \mathrm{H}$ associated nitroreductase activity with paraquat and salicylate treatment in cellular extracts of $E$. coli $\mathrm{AB} 1157$ and its derived mutants.

2.5 ClustalV alignment of $E$. coli $\mathrm{NfsB}$ and the putative NfsB-like homologues.

3.1 (a) NfsB expression vector pITB. Approximate location of 88 mutagenesis target region is indicated. (b) Target region and potential mutations after PCR mutagenesis.

3.2 Illustration of the IMPACT I Purification System.

3.3 Targeted PCR mutagenesis with degenerate primers.

3.4 (a) Double reciprocal plot for a ping-pong $\mathrm{Bi} \mathrm{Bi}$ reaction. Replots as

(b) $1 / \mathrm{Vmax}^{\text {app }}$ versus $1 /[\mathrm{B}]$ and (c) $1 / \mathrm{Km}^{\mathrm{AX}}$ app versus $1 /[\mathrm{B}]$, illustrating the graphical determination of $\mathrm{Km}^{\mathrm{AX}}, \mathrm{Km}^{\mathrm{B}}$, and $\mathrm{Vmax}$ for a ping-pong Bi Bi reaction.

3.5 Expression of the NfsB triple fusion protein at a range of temperatures. 
3.8 Fluorescence spectra.

3.9 Primary double reciprocal plots of initial velocity versus NADH concentration for wild-type NfsB and the mutant enzymes.

3.10 Primary double reciprocal plots of initial velocity versus NADPH concentration for wild-type NfsB and the mutant enzymes.

3.11 Primary double reciprocal plots of initial velocity versus nitrofurazone (NF) concentration for wild-type NfsB and the mutant enzymes.

3.12 Primary double reciprocal plots of initial velocity versus nitrofurazone concentration for wild-type NfsB and the mutant enzymes.

3.13 Secondary double reciprocal plots of Vmax ${ }^{\text {app }}$ versus NADH concentration.

3.14 Secondary double reciprocal plots of $\mathrm{Vmax}^{\text {app }}$ against NADPH concentration.

3.15 Secondary double reciprocal plots of $V \max ^{\mathrm{app}}$ versus nitrofurazone concentration with $\mathrm{NADH}$ as the cofactor.

3.16 Secondary double reciprocal plots of $\operatorname{Vmax}^{\mathrm{app}}$ versus nitrofurazone concentration with NADPH as the cofactor.

3.17 Schematic diagram of a ping-pong reaction mechanism.

3.18 Catalytic specificity of wild-type $\mathrm{NfsB}$ and the mutant enzymes G158S, VAFS and F70S measured as $\mathrm{k}_{\mathrm{cat}} / \mathrm{Km}$.

3.19 Nucleotide sequence of the $n f s B$ gene with the translated sequence below it. Mutagenesis target region is shown in the box.

3.20 Linear replots of slopes at different nitrofurazone concentrations from the double reciprocal plots of $1 / \mathrm{v}$ vs (a) $1 / \mathrm{NADPH}$ and (b) $1 / \mathrm{NADH}$.

3.21 NfsB structure. Phe-70 and the mutagenesis target region from Gly153 to Leu-159 in one chain of the homodimer structure are shown in orange.

4.1 Structural alignment of NfsB and homologues. 
$\begin{array}{ll}4.2 & \text { Solvent accessible pockets of NfsB. } \\ \end{array}$

$\begin{array}{ll}\text { 4.3 Diagrams of the cofactor structures mentioned in this study. } & 175\end{array}$

4.4 Side and front views of the FMN-containing major pocket in (A) NfsB 177 (top) and (B) NIO-bound NfsB (bottom).

4.5 Solvent accessible pockets of NfsB (left) and NIO-bound NfsB (right). 179

4.6 LIGPLOT diagrams showing most of the structural contacts in NfsB 182 with (a) FMN, and (b) NIO.

4.7 (a) Major pocket of NfsB and NIO-bound NfsB. Phe-70 and Phe-124 188 are indicated to illustrate the shift in sidechain position when NfsB is complexed with NIO. (b) Aligned FMN molecules, Phe-70 and Phe124 in NfsB and NIO-bound NfsB.

4.8 NfsA pockets.

4.9 Two views of the aligned NfsA (red) and NfsB (blue) structures.

4.10 Some conformations of NAD. 


\section{List Of ABbREViations}

$\AA$

BLAST

$\mathrm{bp}$

${ }^{\circ} \mathrm{C}$

CFU

$\mathrm{Cnr}$

dATP, dCTP, dTTP, dGTP

ddATP, ddCTP, ddTTP, ddGTP

$\mathrm{ddH}_{2} \mathrm{O}$

DMSO

DNA

dNTP

EDTA

$\mathrm{EtBr}$

FAD

$\mathrm{FMN} / \mathrm{FMNH}_{2}$

$\mathrm{Hfr}$

$\mathrm{hr}$

IPTG

IS
Angstroms

Basic local alignment search tool

Basepair

Degrees Celsius

Colony forming units

Classical nitroreductase (in Salmonella typhimurium)

deoxynucleotide triphosphates (adenine, cytosine, thymidine, guanine)

dideoxynucleotide triphosphates

distilled, deionized water

Dimethylsulfoxide

Deoxyribonucleic acid

deoxynucleotide triphosphate

Ethylenediamine tetra-acetate

Ethidium bromide

Flavin adenine dinucleotide

Flavin mononucleotide/ fully reduced form

High frequency of recombination

hours

Isopropyl-beta-D-thiogalactopyranoside

Insertion sequence 


\begin{tabular}{|c|c|}
\hline $\mathrm{kb}$ & kilobasepair \\
\hline $\mathrm{k}_{\mathrm{cat}}$ & Turnover number, or catalytic velocity \\
\hline $\mathrm{kD}$ & kilodaltons \\
\hline $\mathrm{Km}$ & Michaelis constant \\
\hline $\mathrm{mA}$ & milliampere \\
\hline $\min$ & Minutes \\
\hline MW & Molecular weight \\
\hline $\mathrm{NAD}(\mathrm{P}) \mathrm{H} / \mathrm{NAD}(\mathrm{P})^{+}$ & $\begin{array}{l}\text { Nicotinamide adenine dinucleotide } \\
\text { (phosphate), reduced form/oxidized form }\end{array}$ \\
\hline NEC & NfsB homologue in Enterobacter cloacae \\
\hline NF & Nitrofurazone \\
\hline NFR & Nitrofurazone reductase \\
\hline Nfs & Nitrofurazone sensitivity \\
\hline NfsA & Major NR of $E$. coli \\
\hline NfsB & Minor NR of $E$. coli \\
\hline NIO & Nicotinic acid \\
\hline NR & Nitroreductase \\
\hline PAGE & Polyacrylamide gel electrophoresis \\
\hline PCR & Polymerase chain reaction \\
\hline RNA & Ribonucleic acid \\
\hline $\mathrm{rpm}$ & Revolutions per minute \\
\hline SDS & Sodium dodecyl sulphate \\
\hline TE & Transposable element \\
\hline
\end{tabular}


TNT

UV

V

$\mathrm{V}_{\mathrm{o}}$

$V \max$
Trinitrotoluene

Ultraviolet

Volts

Initial velocity of an enzyme-catalyzed reaction

Maximal velocity of an enzyme-catalyzed reaction 


\section{CHAPTER ONE: GENERAL INTRODUCTION}

\subsection{Nitro-containing compounds}

Nitroheterocyclic and nitroaromatic compounds constitute an enormous range of chemicals that are characterized by the presence of one or more nitro groups on a heterocyclic or aromatic nucleus. For many years nitroaromatic compounds have been used in the industrial production of explosives, pesticides, pharmaceuticals, plastics, and dyes (Drzyzga et al., 1995; Hein, 1988; Spain, 1995). Nitropolyaromatic compounds such as the nitropyrenes have been identified as byproducts of cigarette and food combustion (Salmeen et al., 1982; de Serres and Matsuchima, 1986) and as the principal genotoxic components of diesel, jet fuel, and municipal incinerator emissions (Bond et al., 1985; Eddy et al., 1986; Tokiwa and Ohnishi, 1986), carbon black and toners (Rosenkranz et al., 1980), and urban air particulate (Epstein et al, 1966; Moller et al., 1993).

Human health concern for nitroaromatic compounds has resulted from the identification of several nitro derivatives as potent bacterial mutagens (Lee et al., 1994; Moller et al., 1993; Rosencranz and Mermelstein, 1985; Tokiwa et al., 1981), as mammalian cell mutagens and clastogens (Bauchinger et al., 1988; Li and Dutcher, 1983), and as carcinogens (Cohen, 1978; Howard et al., 1985; King, 1988; Moller et al., 1993; Rosenkranz and Mermelstein, 1983; Tokiwa and Ohnishi, 1986). A further consideration is the potential for synergistic effects on mutagenicity when these compounds are components of complex mixtures (Hass et al., 1987).

Following the Second World War, recognition of the increasing resistance of bacteria to antibiotics led to the investigation of synthetic nitroheterocyclic compounds as 
antimicrobial agents. In particular, the 5-nitrofuran derivatives generated interest, as these broad-spectrum agents were bactericidal and stable in the presence of serum, pus and blood. Moreover, the development of bacterial resistance to such agents appeared to be infrequent, was rarely complete (Paul and Paul, 1964), and was not transmissible to other bacteria (Asnis, 1957; McCalla et al., 1978; Sastry and Jayarman, 1984). For these reasons the 5-nitrofurans experienced widespread use as antimicrobial agents in human and veterinary medicine, and as food additives in the swine and poultry feed industry (Bryan, 1978; McCalla, 1979; Tazima et al., 1975).

The mechanism of activation of nitrofurans is similar to that of the nitroaromatics (i.e. via nitroreduction, discussed in Section 1.2.1), and thus it is not surprising that the nitrofurans have mutagenic and carcinogenic effects as well. Indeed, most nitrofurans are teratogenic, mutagenic, toxic, immunosuppressive and/or carcinogenic (reviewed in Cohen, 1978; McCalla et al., 1971; McCalla, 1979,1980,1983), which has stalled their widespread use as antimicrobial agents. However, many nitrofurans are still used as antimicrobial agents for treatment of infections in which the causative organisms have developed resistance to alternative drugs (Debnath et al., 1993; Johnson et al., 1999; Kwon et al., 2001; Townson et al., 1994). The much more rapid metabolism of nitrofurans by microorganisms (McCalla, 1983) compared to humans makes treatment with these drugs an option when there is no known alternative. Thus, these compounds still represent a promising class of antimicrobial agents that can be developed for the ongoing battle with pathogenic organisms. 


\subsection{Nitroreductases}

The biological activity of nitroheterocyclic and nitroaromatic compounds is derived from reductive metabolism of the parent compound's nitro moiety, a process catalyzed by a variety of nitroreductase (NR) enzymes. Initially, nitroreductases (NRs) were recognized for their ability to reduce the nitro moiety of nitroaromatic and nitroheterocyclic compounds to form biologically reactive products. No other function was ascribed to these enzymes, which distinguished them from other enzymes that exhibited some NR activity in their established physiological roles, such as xanthine oxidase (Wang et al., 1974), NADPH-cytochrome P-450 reductase (Harada and Omura, 1980), NADPH-cytochrome c reductase (Orna and Mason, 1989), and aldehyde oxidase (Wolpert et al., 1973). Genetic characterization of a few established NRs and the advent

of genome projects facilitated the identification of the oxygen-insensitive NRs as a family of enzymes.

\subsubsection{Nitroreduction}

NR activity can be classified on the basis of oxygen sensitivity, a consequence of the enzyme using either one or two electrons to reduce the nitro moiety of the parent compound (Bryant and Deluca, 1991; Eddy et al., 1986; McCalla, 1979; Peterson et al., 1979). In bacteria, reactions catalyzed by type I NRs are oxygen-insensitive, while those mediated by type II NRs are inhibited by the presence of oxygen (Figure 1.1) (Asnis, 


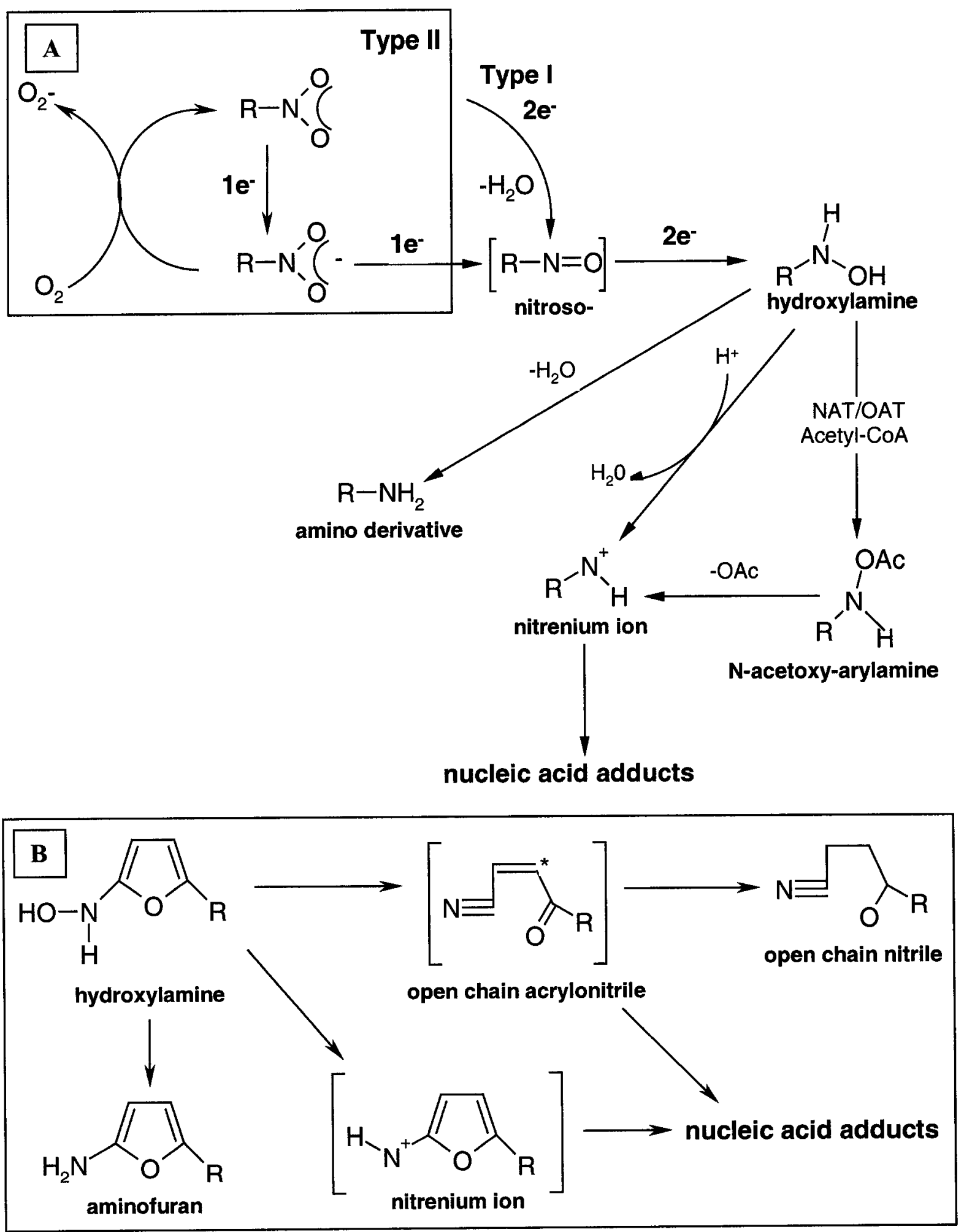

Figure 1.1 (A) The metabolic activation of nitrosubstituted compounds via nitroreduction. (B) Reduction of 5-nitrofurans yields an additional intermediate, the open chain acrylonitrile, which can interact with DNA (refer to text for details) (adapted from Bertenyi and Lambert, 1996). 
1957; Djuric et al., 1986; Mason and Holzman, 1975; McCalla et al., 1975; Peterson et al., 1979).

Oxygen-insensitive nitroreduction appears to occur according to a ping-pong BiBi reaction mechanism (see Section 3.4.1.3), a double displacement reaction that involves the release of a product between the addition of two substrates (Segel, 1975). Metabolism of nitroaromatic compounds proceeding through two-electron reduction of the nitro moiety yields nitroso and hydroxylamine intermediates, and finally an amine product (Gavin et al., 1966; Biaglow, 1981). Metabolism of 5-nitrofuran compounds produces an open-chain nitrile isomer in addition to the aminofuran derivative (Debnath et al., 1993; McCalla, 1979). The endproducts of metabolism of both nitroheterocyclic and nitroaromatic compounds are biologically inactive; the biological activity of these chemicals is conferred by the reactivity of short-lived intermediates with protein, RNA and especially DNA (Djuric et al., 1986; Fifer et al., 1986; Heflich et al., 1986; McCalla et al., 1971; Mermelstein et al., 1981; Novak et al., 1995; Tatsumi et al., 1986; Tu and McCalla, 1975; Wentzell and McCalla, 1980). Nitroreduction that proceeds through single electron increments yields the nitro-anion free radical, at the first step. This radical intermediate can be reoxidized in the presence of oxygen to give the parent nitro substituted substrate and superoxide anion. The futile cycle ensuing from partial reduction and reoxidation of the nitro substituent accounts for the oxygen sensitivity of some biological NRs (Biaglow, 1981; Mason and Holtzman, 1975; McCalla, 1979; Peterson et al., 1979). Figure 1.1 illustrates this mechanism. 


\subsubsection{Characterization of nitroreductases}

Initial investigation of $E$. coli oxygen-insensitive NRs was accomplished through the characterization of nitrofurazone resistant mutants (Bryant et al., 1981; McCalla et al., 1978). Resistance to elevated concentrations of nitrofurazone develops in two distinct steps (McCalla et al., 1970), a characteristic shared by other nitrofurans such as the widely used antimicrobial agents nitrofurantoin (Breeze and Obaseiki-Ebor, 1983; Sastry and Jayarman, 1994) and furazolidone (Whiteway et al., 1998). Similarly, in Helicobacter pylori, resistance to the nitroimidazole compound, metronidazole, occurs through a two step process (Jeong et al., 2000). McCalla and coworkers proposed the existence of two oxygen-insensitive NR enzymes in $E$. coli, encoded by two genes that were referred to as $n f s A$ and $n f s B$, and speculated that the stepwise resistance was the result of the sequential loss of the NR activities due to mutations in these genes (McCalla et al., 1978; Breeze and Obaseiki-Ebor, 1983). In keeping with this speculation is the observation of cross-resistance of mutants between these chemicals (Breeze and ObeisikiEbor, 1983; Whiteway et al., 1998).

Further biochemical characterization led researchers to identify one major (Type $\mathrm{IA}$ ) and two minor (Types $\mathrm{IB}_{1}$ and $\mathrm{IB}_{2}$ ) oxygen-insensitive nitroreductase components (Bryant et al., 1981). Approximately three-quarters of the cellular nitrofurazone (NF) reductase activity in E. coli is attributable to IA, and the remaining one-quarter of cellular activity is ascribed to $\mathrm{IB}_{1} ; \mathrm{IB}_{2}$ contributes negligible NF reductase activity (Bryant et al., 1981; McCalla et al., 1975). Mutants lacking IA and $\mathrm{IB}_{1}$ were easily obtained, and appeared to be encoded by the genes $n f_{s} A$ and $n f s B$, respectively. The gene encoding $\mathrm{IB}_{2}$ 
has not yet been identified. Apart from a study by McCalla et al. (1975) that described a crude gel filtration separation which yielded $700 \mathrm{kDa}$ and $120 \mathrm{kDa}$ fractions containing oxygen-sensitive NR activity, no isolation or characterization of bacterial oxygensensitive NR activity has been accomplished. No mutants have been described for the oxygen-sensitive species, although extensive attempts have been made to isolate such mutants (McCalla et al., 1975). The difficulty that has been encountered with obtaining type II mutants suggests that the gene(s) encoding this oxygen sensitive activity might be essential. Similarly, it has been shown that several enzymes in H. pylori probably have NR activity but appear to be essential for cell survival, as mutants cannot be obtained (Kwon et al., 2000).

A hiatus of nitroreductase research spanned most of the 1980's, but the explosion of genetic and genomic data in the 1990's, particularly the genetic characterization of $n f_{s} B$ in Escherichia coli (Michael et al., 1994), cnr in Salmonella typhimurium (Watanabe et al., 1990a), and $n f n B$ in Enterobacter cloacae (Bryant and DeLuca, 1991), formed the foundation upon which other NRs could be identified by means of sequence comparison. Zenno et al. (1996b) first proposed that this family of enzymes existed, and that it intriguingly included the flavin reductases FRaseI from Vibrio fischeri and Frp from $V$. harveyi. Sequence similarity searches and alignments with the available genomic data yielded putative members of this family in Helicobacter pylori and Bacillus subtilis (Whiteway et al., 1998), which were later confirmed (Jenks et al., 1999; Jorgensen et al., 2001; Sisson et al., 2000; Zenno et al., 1998). Indeed, the NR family is emerging as a widely conserved group of enzymes that has members (either confirmed or putative) in 
10 phyla and 69 species of the three main domains of life: Bacteria ( 53 species), Eukaryota (9 species), and Archaea ( 7 species) (Table 1.1). As is the case in E. coli, many of these organisms appear to contain more than one NR. According to the most recent update (version 7.1, March 2002) of the Pfam Protein Families Database (http://pfam.wustl.edu/), which detects family members in genomic data based on a profile hidden Markov model (Bateman et al., 2002), the nitroreductase/flavin reductase family contains 123 proteins, including four in Eukaryota, 12 in Archaea, and 107 in Bacteria (Table 1.1). Interestingly, the Pfam Protein Families Database identifies four genes in $E$. coli as encoding NR enzymes: $n f s A, n f s B$ and two genes with uncharacterized protein products of approximately $20 \mathrm{kDa}$ each. One or both of these proteins may correspond to the remaining oxygen-insensitive component $\mathrm{IB}_{2}$. It may also be that these genes are paralogs of $n f s B$, serving a different function but arising from past gene duplication (Chen and Jeong, 2000).

The Pfam database includes one human hypothetical protein as a NR family member. In addition to this hypothetical protein, humans express $\mathrm{NAD}(\mathrm{P}) \mathrm{H}$ :quinone reductase (NQO1), also known as DT-diaphorase, which is biochemically similar to the oxygen-insensitive NRs of $E$. coli (see Section 1.2.2.1), but does not share any sequence similarity with the bacterial NRs. Including NQO1, there are 19 putative homologous enzymes that may be added to the Pfam list of proteins in the NR family on the basis of biochemical evidence. The organisms in which these proteins have been identified are noted in bold in Table 1.1 and include fungi (McCoy et al., 1984; Rieble et al., 1994) and three species of marine invertebrates (Hetherington et al., 1996). 


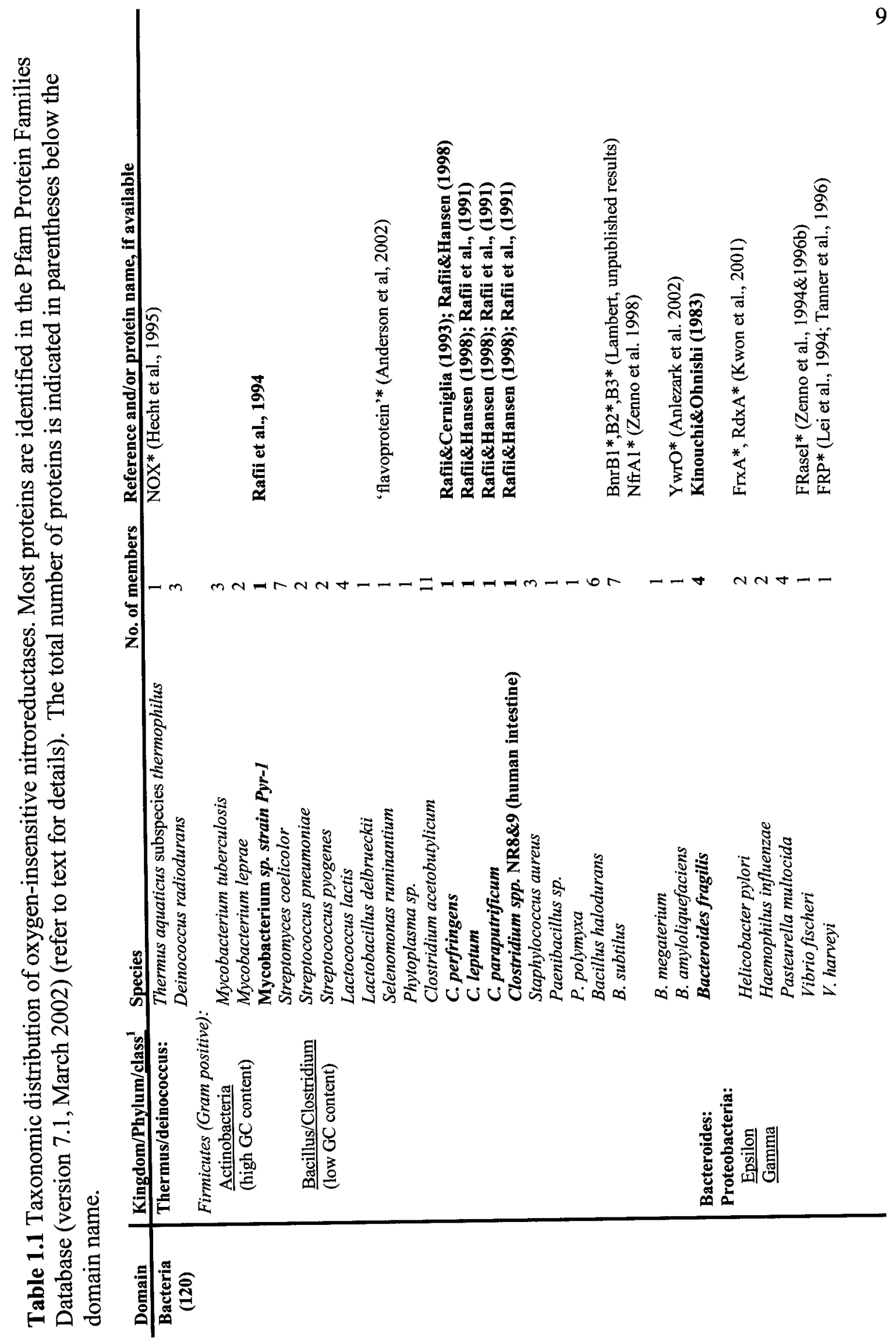




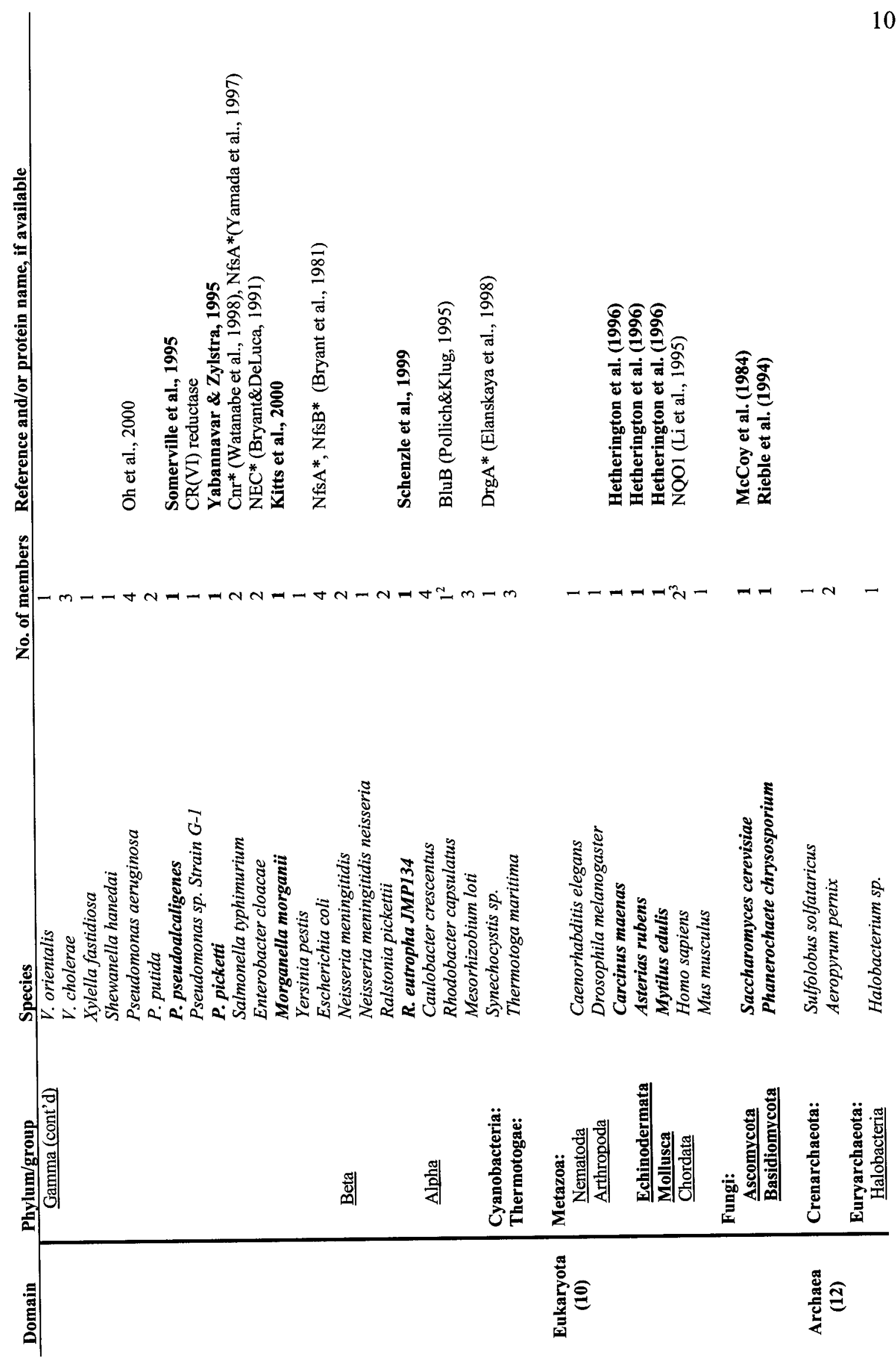




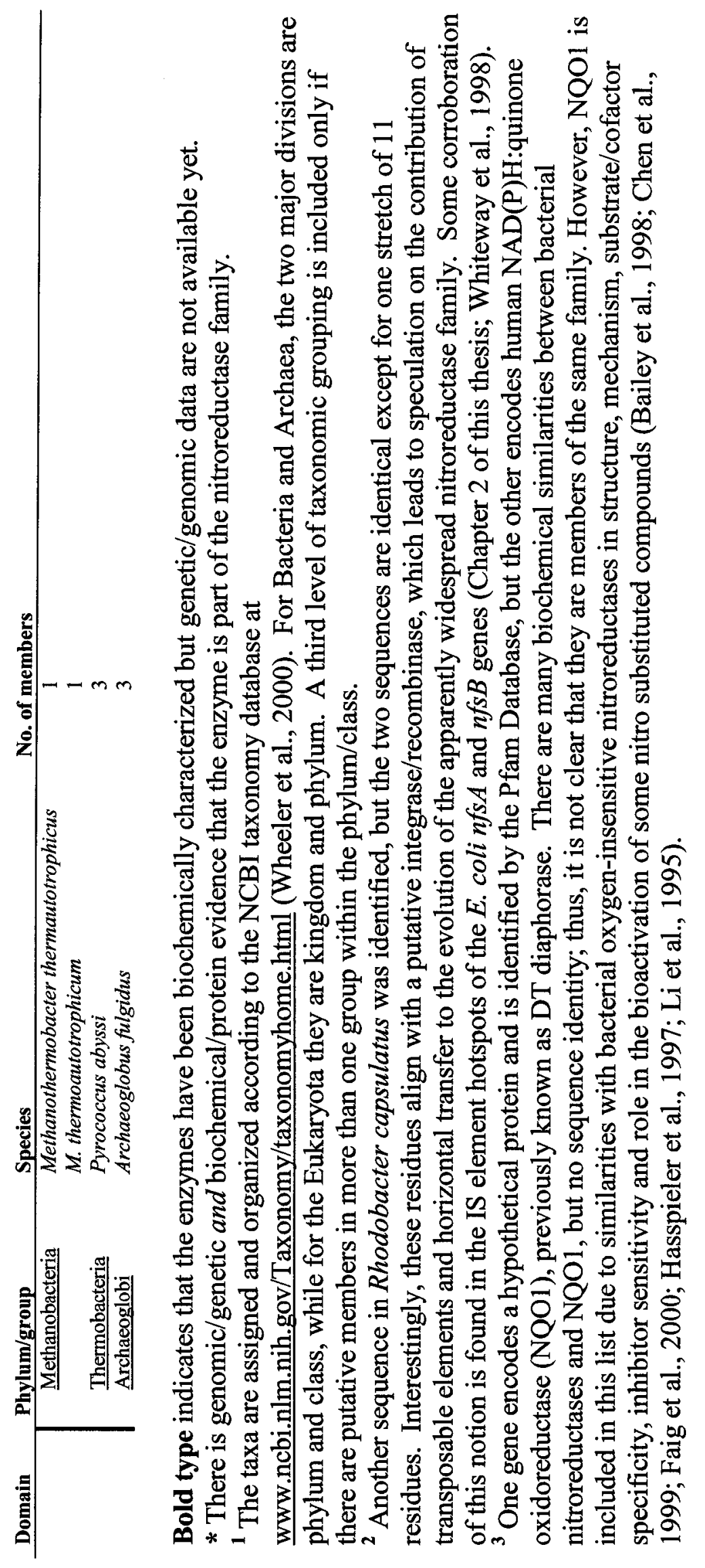


The flavin reductases within the NR family provide reduced $\mathrm{FMNH}_{2}$ for the luminescence reaction in Vibrio species, but the physiological role(s) of the nitroreductases remains a mystery. There is some evidence that NfsA and NfsB of $E$. coli (Liochev et al., 1999; Paterson et al., 2002), and the NfsA-like protein of Salmonella typhimurium (Nokhbeh et al, in press; Pomposiello and Demple, 2000) are involved in the cellular response to oxidative stress as part of the SoxRS regulon. NfsA and NfsB also appear to be members of the MarRAB regulon, which suggests that they are part of the global stress response that is typically associated with multiple antibiotic resistance (Barbosa and Levy, 2000). Interestingly, the putative homologues of the $E$. coli oxygeninsensitive NRs in Caulobacter crescentus have a distinct cell cycle expression profile, with the highest expression occurring during G1 (Grünenfelder et al., 2001). This is similar to several other proteins that are employed in the oxidative stress response, which the researchers suggest is a mechanism to suppress DNA damage during replication.

Recently, structural characterization of NRs has gained momentum. Threedimensional (3D) structures have been resolved by X-ray crystallography for six of the bacterial enzymes (refer to Chapter 4, Table 4.1 for the PDB identifiers of these structures): E. coli NfsA (Kobori et al., 2001) and NfsB (Parkinson et al., 2000; Loverling et al., 2001), E. cloacae NEC (Hecht et al., to be published), $V$. fischeri FRaseI (Koike et al., 1998), V. harveyi FRP (Tanner et al., 1996), and T. thermophilus NOX (Hecht et al., 1995). Furthermore, some of these structures have been resolved with cofactor analogues and inhibitors. 


\subsubsection{Characteristics of the NR family proteins}

A protein family groups together proteins of similar sequence and domain structure (Murzin et al., 1995) that usually have the same function in various organisms, and are assumed to share evolutionary origin (Barker et al., 1996). According to the Structural Classification of Proteins (SCOP) version 1.59 (released May 2002) (Murzin et al., 1995), NRs belong to the NADH oxidase/flavin reductase family. However, in the literature and other sources such as the previously mentioned Pfam Database, this family is more often referred to as the NR family.

Enzymes in the oxygen-insensitive NR family are flavoproteins, homodimers of approximately $50 \mathrm{kDa}$, with one tightly bound molecule of FMN (sometimes FAD) per monomer. $\mathrm{NAD}(\mathrm{P}) \mathrm{H}$ is utilized as the source of reducing equivalents, in the form of hydrides, which reduce the bound flavin molecule. In an obligatory 2-electron transfer mechanism, the reduced flavin subsequently reduces the nitro containing substrate. No semiquinone state of the bound flavin has been observed for enzymes in the NR family. This type of reaction mechanism is referred to as ping-pong, as it is a displacement reaction in which a product is released (i.e. oxidized $\left.\mathrm{NAD}(\mathrm{P})^{+}\right)$between the addition of substrates (refer to Section 3.4.1.3 for more details).

Enzymes in the NR family largely share substrate specificities, with the notable exception of the flavin reductases of $V$. fischeri and $V$. harveyi. Neither NfsA nor NfsB is an effective flavin reductase. However, Zenno et al. (1996a \& b) demonstrated that a single missense mutation in NfsA and NfsB resulted in each enzyme being an efficient flavin reductase, which corroborates an orthologous relationship for these proteins. 
In addition to the biochemical and kinetic characterization of proteins in the NR family, six 3D structures of bacterial NRs have been resolved (see Section 1.2.2). All six proteins are classified as $\alpha+\beta$ type proteins in the SCOP database, a classification that refers to the segregated $\alpha$-helix and mainly antiparallel $\beta$-sheet regions that comprise the proteins. The 3D structure of human NQO1 (see Section 1.2), which is not included in the SCOP NR family, has also been resolved. NQO1 is also a $60 \mathrm{kDa}$ homodimer, with a bound FAD molecule in each monomer, and is classified as an $\alpha+\beta$ type protein. It uses $\mathrm{NAD}(\mathrm{P}) \mathrm{H}$ as a source of reducing equivalents for the obligatory 2-electron reduction of nitro containing compounds according to a ping-pong reaction mechanism.

\subsubsection{The NR family can be divided into two subgroups}

Within the NR family the enzymes can be divided into two main groups. Here, these will be referred to as NfsA-like and NfsB-like, according to the $E$. coli enzymes. There is little sequence identity between these two groups of enzymes; NfsA and NfsB share approximately $16 \%$ identity, although due to some highly conserved residues and regions (discussed in more detail in Chapter 4) sequence identity can be used to define them as part of the NR family. Biochemically, the primary distinction between the NfsAlike and NfsB-like enzymes is cofactor usage. The NfsA-like proteins are NADPHdependent, while the majority of the NfsB-like enzymes can use both NADH and NADPH.

Despite overall sequence dissimilarity and the difference in cofactor specificity, the structures of NfsA and of FRP, the NfsA-like protein of $V$. harveyi, are similar to 
those of NfsB and the NfsB-like proteins. There are a few revealing differences that can be used to distinguish them, however, which are described in Chapter 4 (Section

\subsection{4).}

\subsubsection{NfsB}

The $n f s B$ gene (also referred to as $n f n B, n f s I, n t r$ and $d p r a$ ) in $E$. coli maps to 13 min. on the chromosome (Blattner et al., 1997; McCalla et al., 1978). Little is known about the regulatory control of $n f s B$. There are putative Shine-Delgarno, -10 and -35 sequences in the 5'-untranslated region of the gene, but these have not been experimentally confirmed. The $n f s B$ gene appears to be regulated by MarA, the transcriptional activator of the multiple-antibiotic-resistance (mar) locus in $E$. coli (Barbosa and Levy, 2000), and is possibly part of the soxRS regulon (Liochev et al., 1999; Chapter 2) which suggests that regulatory sites are present within this region, even though a consensus sequence for a 'soxbox' or 'marbox' has not been identified. Some regulatory control of the $E$. cloacae $n f n B$ gene, encoding the NEC protein, is also apparent, as the protein is induced up to ten-fold by exposure to 2,4,6-trinitrotoluene (TNT) (Bryant and DeLuca, 1991).

The $n f s B$ gene encodes $\mathrm{NfsB}$, a FMN-containing flavoprotein that corresponds to the minor oxygen-insensitive nitroreductase component $\mathrm{IB}_{1}$ previously characterized in $E$. coli (Bryant et al., 1981). NfsB is composed of 217 amino acids with a monomeric molecular mass of approximately $24 \mathrm{kDa}$ and can use either NADH or NADPH as a source of reducing equivalents. NfsB shares $88 \%$ amino acid sequence identity with homologous NRs isolated from Salmonella typhimurium (Watanabe et al., 1990a) and E. 
cloacae (Bryant et al., 1991), 32\% identity with FRaseI, the major flavin reductase of $V$. fischeri (Zenno et al., 1996b), and at least 20\% identity with NfsB-like bacterial orthologues. The identification of NR homologues is largely based on the identity of conserved regions in NfsB, especially those spanning Arg-10 to Asp-17, Ser-37 to Val50, and Gly-158 to Asp-168 (refer to Figure 1.2). To varying degrees each of these regions is involved in FMN binding, but some are also unique to the NfsB-like NRs, particularly the $\mathrm{P}^{45}$ WHFIV ${ }^{50}$ sequence. While the conserved residues in NRs are informative, the region from approximately Phe-108 to Glu-137 in NfsB is interesting for its lack of conservation. This region most likely contributes to the flexibility of the enzyme that is necessary to accommodate the wide variety of nitro-substituted substrates. Indeed, a point mutation of Phe-124 to Ser significantly increases the catalytic specificity of NfsB for FMN, to a level comparable to that of FRaseI, the NfsB homologue in $V$. fischeri (Zenno et al., 1996c).

\subsection{Applications of $\mathrm{NfsB}$}

The characterization of $\mathrm{NfsB}$ is useful for many applications including drug development (Section 1.3.1.1), cancer therapy (Section 1.3.1.2), and genetic toxicology (Section 1.3.2.1). A thorough understanding of the structure-function relationships of $\mathrm{NfsB}$ can be an important element in the optimization of these applications. Knowledge of essential residues for enzyme activity and specificity, and an understanding of the mechanism(s) of nitroreduction will maximize the biotechnological potential of $\mathrm{NfsB}$. The growing number of patents that include nitroreductases and/or their gene sequences exemplifies the commercial potential of nitroreductases: since 1996, the US Patent 
Figure 1.2 Alignment of characterized NfsB-like nitroreductases. The boxes noted as 'conserved' indicate the three conserved regions spanning Arg-10 to Asp-17, Ser-37 to Val-50, and Gly-158 to Asp-168 in NfsB that are mentioned in the text (Section 1.2.3). The 'not conserved' box outlines a region that is not well conserved in homologous enzymes and is proposed to be involved in substrate binding (Section 4.4.2.3 this thesis; Haynes et al., 2002; Koike et al., 1998; Loverling et al., 2001; Zenno et al., 1996b). The black line beneath the alignment from Pro-45 to Val-50 of the NfsB sequence indicates the NfsB specific region, especially the Trp-46 residue that is completely conserved in all characterized NfsB-like proteins, but is not present in the NfsA-like proteins. 


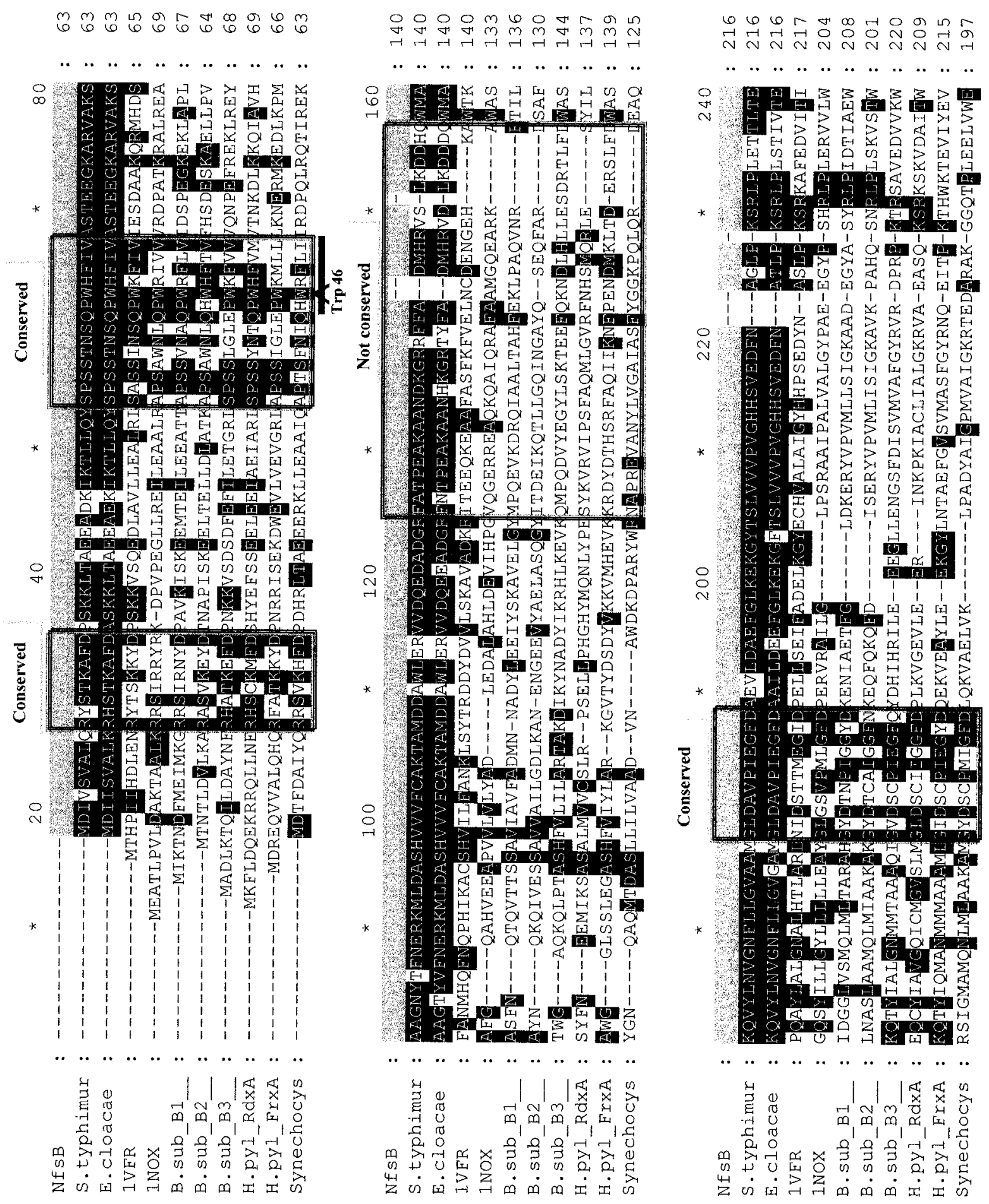


and Trademark Office lists 75 patents involving nitroreductases; the European Patent Office and the Canadian Intellectual Property Office list 35 and 28 patents, respectively. Most of these patents involve the directed enzyme prodrug approach for cancer therapy (Section 1.3.1.2).

\subsubsection{Therapeutic Applications}

\subsubsection{Antimicrobial mechanisms and drug development}

The characterization of $\mathrm{NfsB}$ and other NRs has promoted a greater understanding of bacterial pathways of resistance to antimicrobial agents (Debets-Ossenkopp et al, 1999; Elanskaya et al., 1998; Goodwin et al., 1998; Jenks et al., 1999; Jorgensen et al., 2001; Kwon et al., 2000, 2001a\&b; Sisson et al., 2000, Tankovic et al., 2000; Whiteway et al., 1998). Mechanistic knowledge of nitroheterocyclic drug metabolism is significant as it could lead to the design of new highly effective drugs with minimal side effects (Biaglow, 1981) and less chance for development of resistance. Furthermore, structurefunction knowledge can lead to the design of drugs that are more specific for an enzyme, or, conversely, an enzyme that is more compatible with a drug (discussed in Chapter 4). Indeed, some investigations into alternative drugs that are activated by NfsB have been undertaken in order to obtain an optimal prodrug for use in directed enzyme prodrug therapy (DEPT) cancer therapy approaches (see Section 1.3.1.2) (Atwell et al., 1996; Bailey et al., 1996).

\subsubsection{Cancer Therapy}

The toxicity of anticancer drugs is primarily due to their lack of specificity for malignant cells. An anticancer agent can only be effective if a sufficiently high 
concentration is achieved at the target site; therefore a great deal of research has focused on selectively targeting tumour cells. Directed enzyme prodrug therapy (DEPT) is a promising method for the selective delivery of cytotoxic anticancer agents to cancer cells. This approach separates the targeting and cytotoxic functions of an anticancer agent by first delivering a foreign enzyme to the target site where it can bioactivate a subsequently administered non-toxic prodrug. $\mathrm{NfsB}$ is an excellent candidate for the local generation of a cytotoxic drug as the nitro group of a number of compounds can act as a 'trigger' for drug bioactivation. In addition, since NfsB is a bacterial enzyme, it is unlikely that NfsB substrates will be prematurely activated by a homologous host enzyme.

The most popular choice of prodrug for NfsB has been the DNA crosslinking agent 5-(aziridin-1-yl)-2,4-dinitrobenzaminde (CB1954) (Anlezark et al., 1992; Knox et al., 1993). CB1954 is preferred to other widely used prodrugs that target DNA synthesis, as it is not dependent on a cell-cycle phase for its cytotoxicity. Furthermore, CB1954 is a poor substrate for human NQO1 (see Sections 1.2 and 1.2.2.1), but is rapidly reduced by NfsB (Anlezark et al., 1992).

Two main approaches are being explored to deliver NfsB, and other suitable bioactivating enzymes, to solid tumours. One is antibody-directed enzyme prodrug therapy (ADEPT), which uses an antibody-enzyme conjugate for delivery. The other is the so-called 'suicide' gene therapy approach, generally referred to as gene-directed enzyme prodrug therapy (GDEPT), in which the gene that expresses the foreign enzyme is delivered to the tumour site. ADEPT was initially explored as the delivery system for 
NfsB (Anlezark et al., 1995; Bailey et al., 1996), but GDEPT has now been adopted as the preferred approach for NfsB delivery (Bailey et al., 1996; Bridgewater et al., 1995; Djeha et al., 2001; Green et al., 1997; Nishihara et al., 1998; Weedon et al., 2000). Table 1.2 lists the studies that have investigated the NfsB/CB1954 GDEPT pair, and the vectors that have been used to deliver $n f s B$.

In vitro studies favourably compare $\mathrm{NfsB} / \mathrm{CB} 1954$ to alternative prodrug activating pairs (Grove et al., 1999). In vivo, the NfsB/CB1954 pair has been shown to be effective against a variety of cancers and tissue types (refer to Table 1.2), with a clear bystander effect (i.e. the ability to kill surrounding cells) and even regression of tumours leading to increased survival and some apparent cures in mice (McNeish et al., 1998; Wilson et al., 2002). DEPT is a relatively new approach in cancer therapy and until 1999 only two enzyme/prodrug pairs had been evaluated in clinical trials (Aghi et al., 2000). In the UK, five applications for clinical trials involving nitroreductase have been approved since 1998, although only two are actually ongoing (see Table 1.2) (ChungFaye et al., 2001; Djeha et al., 2001).

\subsubsection{Detection and analysis of nitro-containing compounds}

The specificity of $\mathrm{NfsB}$ and other nitroreductases for ubiquitous nitroaromatic environmental contaminants makes them well suited for applications that involve the assessment of nitro compounds. Currently, NRs are widely exploited in genotoxicity assays (Section 1.3.2.1), and they also appear to have potential for the routine detection and quantification of TNT (Section 1.3.2.2). 


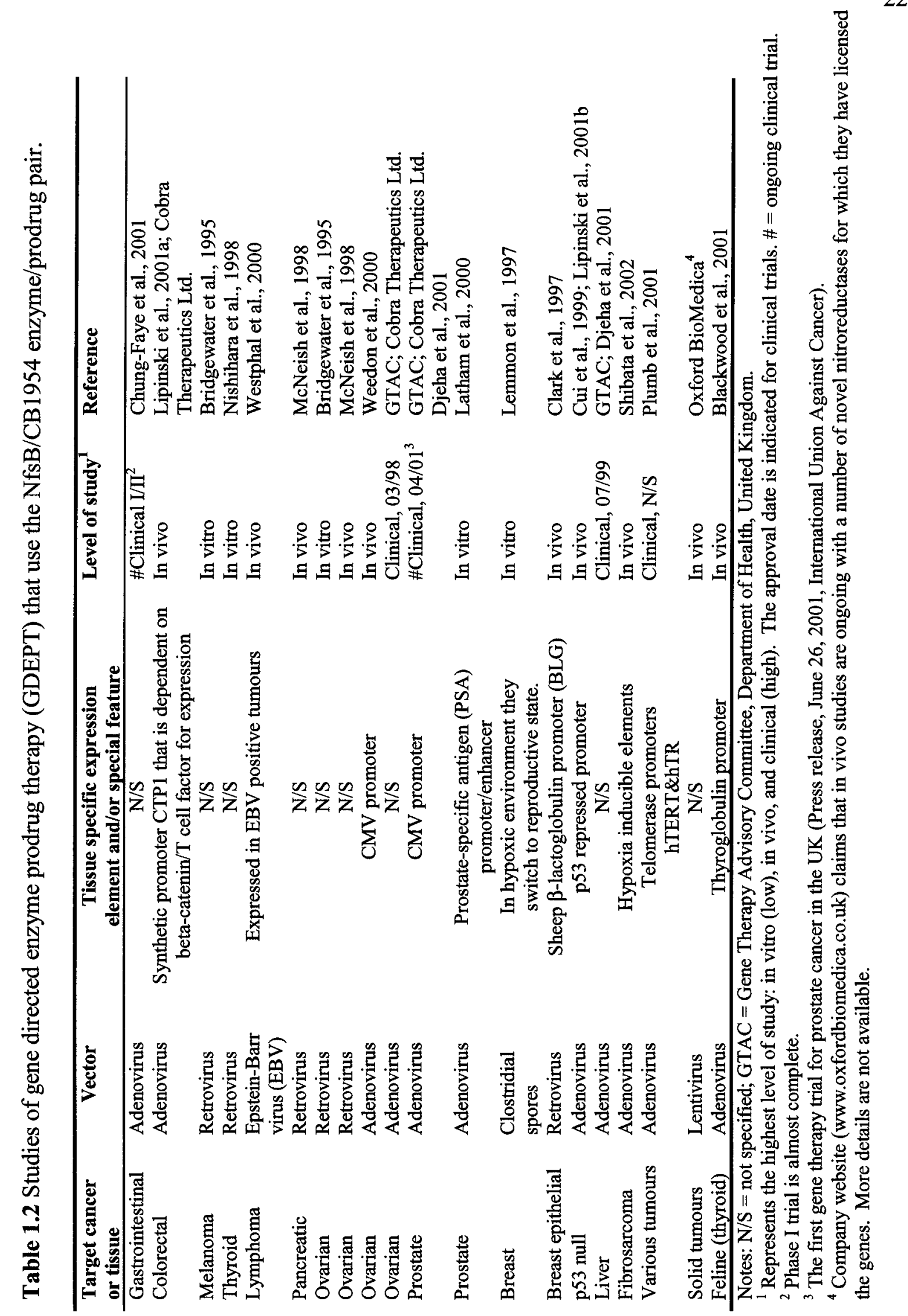




\subsubsection{Genetic toxicology}

Bacterial genotoxicity assays have been widely used for over two decades to identify genotoxic hazards and elucidate mechanisms of mutagenesis. A number of assays exist, including commonly used Salmonella typhimurium his' reversion assay, or Ames test (Ames, 1979), and the E. coli lacZ reversion mutagenicity assay (Cupples and Miller, 1989). The Ames test has been made much more sensitive in the detection of certain carcinogens through the construction of $S$. typhimurium nitroreductase (Cnr) overproducing strains (Watanabe et al., 1989). Recently, several S. typhimurium tester strains were created that express the $E$. coli $\mathrm{Nfs} A$ and $\mathrm{NfsB}$ proteins, and sensitivity to some compounds has been shown to be up to two orders of magnitude greater compared to strains containing only the S. typhimurium genes (Carroll et al., 2002). The flexibility afforded by a number of different NRs of varying activities is key to the development of sensitive and informative tester strains. The value of sensitive tester strains is threefold. First, heightened sensitivity facilitates detection of mutagens thereby requiring less sample for the testing process (Hagiwara et al., 1993; Josephy et al., 1997; Watanabe et al., 1989). Second, the combined application of these strains with isogenic tester strains of various metabolic capacities provides a means for the initial classification of mutagens (Einisto et al., 1991; Josephy et al., 1997; Jurado and Pueyo, 1995). It should be noted that some commonly used S. typhimurium tester strains (TA1535, TA1538 and their derivatives) are not isogenic with respect to NRs, as was previously thought (Yamada et al., 1997), and that the recent flood of genome data should help improve the precision of current genotoxicity assays. Finally, the metabolic activation pathway of a chemical 
agent can be elucidated through the application of particular sets of strains (Hatcher et al., 1995; Josephy et al., 1997; Watanabe et al., 1989; 1990b). The characterization of $\mathrm{NfsB}$ and the ability to manipulate its sensitivity could be a means to contribute to the development of more informative tester strains. Furthermore, as evident from the new $S$. typhimurium tester strains that use $E$. coli NRs (Carroll et al., 2002), homologous enzymes from other organisms are a resource for the development of additional strains. For example, the expression of the $H$. pylori homologue $\mathrm{RdxA}$ in the naturally metronidazole-insensitive $E$. coli leads to metronidazole sensitivity (Sisson et al., 2000).

\subsubsection{Biosensors}

Biosensors, where biological materials are used as sensing components, are gaining attention as a means to specifically and sensitively detect compounds of environmental or health concern. Recently, an amperometric biosensor was constructed using the NEC enzyme of $E$. cloacae to detect low levels of TNT (Naal et al., 2002). The rapid quantification of TNT and its degradation products was also achieved by means of a classical assay that utilizes partially purified NR(s) from Pseudomonas aeruginosa (Oh et al., 2000). Both of these techniques represent alternatives to the HPLC method that is currently used, which is unsuitable for large numbers of simultaneous samples.

NRs, with their characteristically broad substrate specificity, are well suited for the detection of nitro compounds. Furthermore, it is possible that, as described in Section 1.3.3 for TNT, the NRs can be subsequently employed to detoxify the contaminant and hence remediate the polluted environment. 


\subsubsection{Bioremediation}

In 1998, the world market for remediation was between \$US15 and 18 billion (Watanabe, 2001). An expensive and time-consuming process, remediation efforts usually focus on ground water and soil, and involve moving the contaminant in its unchanged form to another site. Phytoremediation, a rapidly growing approach that uses plants to remove contaminants (Meagher, 2001), addresses this challenge. Indeed, plants serve as both pump and treatment system: the extensive root systems of plants can extract from the soil and groundwater compounds which can then be metabolized within the plant.

TNT and its natural breakdown products are toxic and fairly stable, thereby representing a significant environmental contaminant. Recently, Bruce's lab constructed transgenic tobacco plants bearing the $n f s B$-like gene from $E$. cloacae (Hannick et al., 2001). As mentioned in Section 1.2.3, in E. cloacae, the NfsB-like protein (NEC) is induced up to 10 times in the presence of TNT (Bryant and DeLuca, 1991), a property that is arguably (Koder et al., 2001) ascribed to the isolation of the bacterium from a munitions dump. The NR-expressing plants displayed an increase in TNT uptake and detoxification, and the lab is now investigating the use of this gene in more suitable phytoremediation plants such as poplars, which are deep rooted and fast growing (Hannick et al., 2001).

The broad substrate specificity of NRs, particularly with ubiquitous environmental contaminants, lends itself to bioremediation efforts. Thorough 
characterization of the NR proteins, their substrate specificity, and mechanism can aid in the development of specific bioremediation strategies.

\subsection{Rationale and Research Objectives}

At the initiation of this study, there was little research pertaining to NRs: some biochemical characterization was achieved prior to 1980 , and three bacterial $n f s B$ gene sequences were available. NRs were known to be key activating enzymes in the Ames test, and NfsB was being explored as a possible candidate for directed enzyme prodrug therapy. The rationale of this study was to use a molecular biology approach to elucidate the role of the $n f_{s} B$ gene in the development of resistance to nitroheterocyclic antimicrobial agents and to gain an understanding of the structure-function relationships of the NfsB protein.

The project is divided into four main research objectives and hypotheses.

\subsubsection{How does resistance to 5-nitrofurans develop in E. coli?}

\subsubsection{Hypothesis}

The observation that bacterial resistance to antimicrobial nitroheterocyclic agents occurs in a stepwise manner is most consistent with the notion that increased resistance is associated with sequential mutagenic inactivation of multiple NRs (Bryant et al., 1981; McCalla et al., 1978). The hypothesis of this study was that mutations in the $E$. coli genes encoding NfsA and NfsB result in the development of $E$. coli resistance to 5-nitrofurans.

\subsubsection{Objectives}

Several nitrofurazone resistant mutants that were well characterized in earlier studies (Bryant et al., 1981; McCalla et al., 1978), and several furazolidone resistant 
mutants isolated in our lab were used to investigate this hypothesis by two approaches. First, biochemical characterization of the wild-type and mutant strains was achieved by means of a cellular extract assay for nitrofurazone reductase activity; and second, DNA sequence analysis was used to identify mutations in the $n f_{s} B$ gene.

\subsubsection{Is NfsB part of the Sox/Mar response to environmental stress?}

\subsubsection{Hypothesis}

In $E$. coli, the soxRS regulon is the key mediator of the oxidative stress response (Liochev et al., 1999), while the closely related marRAB regulon can lead to enhanced resistance to multiple antibiotics (Barbosa and Levy, 2000; Martin et al., 1999). There is a substantial overlap of genes regulated by the $\operatorname{sox} R S$ and marRAB regulons (Martin et al., 1999). Indeed, the $n f s A$ gene is a member of the soxRS regulon (Liochev et al., 1999; Paterson et al., 2002), and is activated by the MarA protein of the marRAB regulon (Barbosa and Levy, 2000). The MarA protein also activates the $n f_{s} B$ gene (Barbosa and Levy, 2000), but it is not known whether $n f_{s} B$ is a member of the soxRS regulon. The hypothesis of this study was that $n f S B$ is a member of both the marRAB and $\operatorname{sox} R S$ regulons.

\subsubsection{Objectives}

To test this hypothesis, the induction of NfsB-specific NR activity was analyzed in cellular extracts after treatment of the bacterium with paraquat and salicylate, which specifically stimulate the SoxRS and MarRAB regulons, respectively. A comparison of NADH-associated NR activity in four strains of $E$. coli with differing $n f_{s} A n f_{s} B$ genotypes identified NfsB-specific NR activity. 


\subsubsection{What are the determinants of cofactor specificity in $\mathrm{NfSB}$ ?}

\subsubsection{Hypothesis}

$\mathrm{NfsB}$ utilizes $\mathrm{NAD}(\mathrm{P}) \mathrm{H}$ as a source of reducing equivalents, but while the $3 \mathrm{D}$ structure of NfsB has been resolved (Parkinson et al., 2000), the binding site for $\mathrm{NAD}(\mathrm{P}) \mathrm{H}$ was not determined and there is no obvious consensus sequence. The seven residue region of NfsB spanning Gly-153 to Leu-159 (GVAALGL) shares some similarity with known $\mathrm{NAD}(\mathrm{P}) \mathrm{H}$ binding sites. It was hypothesized that missense mutations introduced to this region may alter the cofactor specificity of $\mathrm{NfsB}$, thereby indicating its involvement in $\mathrm{NAD}(\mathrm{P}) \mathrm{H}$ binding. The fact that $\mathrm{NfsB}$ has the unusual property of using both NADH and NADPH as cofactors was considered an asset, as any disparity in the relative specificities could be attributable to the 2'-phosphate of NADPH.

\subsubsection{Objectives}

Four primary experimental objectives were designed to test this hypothesis. First, a proficient system of protein expression and purification was developed using the IMPACT $^{\text {TM }}$ I system from New England Biolabs Ltd.. Second, the wild-type enzyme was kinetically characterized to determine its reaction mechanism and kinetic parameters for NADH, NADPH and nitrofurazone. Third, a PCR mutagenesis approach was used to introduce missense mutations to each position in this region. Finally, the mutant enzymes were purified and kinetically analyzed and the cofactor specificities were compared with those of wild-type NfsB. 


\subsubsection{What can computer analysis reveal about the active site of $\mathrm{NfSB}$ ?}

\subsubsection{Hypothesis}

Computer analysis of 3D protein structures is becoming increasingly more useful and routine with the growing number of powerful applications that are accessible to the average user. It was hypothesized that a comparison of the $3 \mathrm{D}$ structure of $\mathrm{NfsB}$ with homologous structures can elucidate fundamental aspects of the $\mathrm{NfsB}$ active site, particularly in conjunction with the kinetic and mutant analysis obtained from preceding studies. This notion was extended to predict regions of $\mathrm{NfsB}$ that could be recruited for drug or ligand development.

\subsubsection{Objectives}

A four-step approach was used to test this hypothesis. First, a structural alignment of the six proteins for which 3D structures are available was generated. Second, known ligand-protein contacts were determined for each enzyme and their positions in the $3 \mathrm{D}$ structures was compared. The structural contacts of other key residues in NfsB were also determined. Third, protein pockets were identified and the main active site pockets of $\mathrm{NfsB}$ were defined. The residues in these pockets not involved in FMN binding were considered likely candidates for NAD(P)H and substrate binding. Fourth, some of the mutant enzymes generated from previous studies were modeled and the available kinetic and structural data were harmonized with the computer analyses. 


\section{CHAPTER TWO: THE DEVELOPMENT OF 5-NITROFURAN RESISTANCE IN $E$. COLI}

\section{$\underline{2.1 \text { Introduction }}$}

\subsubsection{Nitroheterocyclic drugs}

Since their discovery in the 1940 's, nitroheterocyclic drugs have been used extensively in clinical and veterinary medicine (McCalla, 1979) and as food preservatives (Aoki et al., 1975; De Serres, 1974). Thousands of nitroheterocyclic antimicrobial agents have been synthesized, though only a few have been used therapeutically (HamiltonMiller and Brumfitt, 1978). Of these, the 5-nitroimidazoles and 5-nitrofurans have been especially successful as antimicrobial agents due to broad spectrum activity, high biological stability and a low acquisition of resistance by microorganisms (Bryan, 1978; Hof et al., 1987; McCalla, 1979, 1983) that is apparently not transferred to other microorganisms (Asnis, 1957; McCalla et al., 1978; Sastry and Jayarman, 1984). Nitroheterocyclic drugs can be effective against Gram negative and positive bacteria, protozoa, fungi and even worms (Hof et al., 1987).

The hope that nitroheterocyclic drugs would be the answer to the growing spectre of microbial antibiotic resistance was diminished by the discovery of the toxic, mutagenic, clastogenic, and carcinogenic effects of many of these compounds (Debnath et al, 1993; McCalla, 1983; Tu and McCalla, 1975). Nitrofurans are usually more mutagenic than nitroimidazoles (Tocher, 1997). There are a number of commonly used nitroimidazole drugs (e.g. metronidazole), but only three of the original 5-nitrofuran derivatives are currently used as antimicrobial agents. Nitrofurazone, the first compound to be introduced, is used for bladder irrigation and as a preventive agent against catheterassociated urinary tract infections (Johnson et al., 1999). Nitrofurantoin is a routine 
treatment for urinary tract infections (Debnath et al, 1993). Furazolidone is often used for the treatment of gastrointestinal infections, including those due to protozoa (e.g.. giardiasis, trypanosomiasis), as a treatment for swine and poultry, and as an antimicrobial agent for diseases that have developed multi-drug resistance to other antibiotics (Townson et al., 1994).

Despite the evident mutagenicity and carcinogenicity of many nitroheterocyclic antimicrobial agents, research and synthesis of novel nitro-substituted compounds, including nitrofuran derivatives, continues. Their effectiveness as broad spectrum antimicrobial agents provides the motivation for such efforts, and the large variety of compounds that can be synthesized through variation of the location and number of nitro group substitutions, allows for the design of numerous candidate drugs that may exhibit reduced toxicity and mutagenicity (Townson et al., 1994). Furthermore, in eukaryotic cells the mutagenicity of many nitro-substituted derivatives is often weak and occurs primarily under anaerobic conditions, suggesting metabolic activation by oxygensensitive enzymes. Indeed, exploitation of the anaerobic activation of nitro-substituted compounds such that they can serve as hypoxic-specific cytotoxins and as hypoxic indicators is being explored (Tocher, 1997).

\subsubsection{Metabolic activation of nitroheterocyclic drugs}

The biological activity of nitroheterocyclic drugs depends upon reductive metabolism of the nitro group attached to the imidazole or furan ring, although the metabolism of these drugs was thought to be via different pathways: the nitroimidazoles through reduction by ferredoxin or flavodoxin, and the nitrofurans by nitroreductase 
activity (Townson et al., 1994). However, recent studies on the development of resistance to the nitroimidazole compound metronidazole (Goodwin et al., 1998; Jeung et al., 2000) suggest that nitroreductase activity is also involved in the biological activation of this compound.

In $E$. coli, oxygen-insensitive nitroreductase activity can mostly be ascribed to $\mathrm{Nfs} \mathrm{A}$ and $\mathrm{NfsB}$, with some negligible activity associated with a third uncharacterized component (refer to Chapter 1, Section 1.2.2). Approximately three-quarters of oxygeninsensitive nitroreductase activity is attributable to $\mathrm{NfsA}$, and the remaining one-quarter to NfsB (Bryant et al., 1981). NfsB can use either NADH or NADPH as a source of reducing equivalents, while NfsA can only utilize NADPH as a cofactor.

\subsubsection{Microbial resistance to 5-nitrofurans}

Many of the early insights regarding nitroreductase enzymes were obtained through the biochemical characterization of nitroreductase mutants (Bryant et al., 1981; McCalla et al., 1970, 1978). The observations that bacterial resistance to antimicrobial nitroheterocyclic agents occurs in a stepwise manner is most consistent with the notion that increased resistance is associated with the sequential inactivation of multiple nitroreductases. Although $E$. coli genes encoding the Nfs A and NfsB nitroreductases have now been identified, the direct link between mutation in these genes and the development of bacterial resistance has yet to be established. To address this point, the DNA sequence alterations in the $n f s A$ and $n f s B$ genes of a large number of first and second step furazolidone resistant mutants and previously isolated nitrofurazone resistant mutants were analyzed in this study, and the cellular nitroreductase activities of the 
mutants were determined. The results suggest that sequential resistance to nitrofurans in $E$. coli is associated with a first step event resulting in the inactivation of the $n f s A$ gene, and a subsequent mutational event within the $n f_{s} B$ gene.

While inactivation of these genes leads to nitrofuran resistance, the physiological roles of NfsA and NfsB still remain a mystery. However, the reductive metabolic activity of the nitroreductase enzymes suggests a protective role. Indeed, recent research demonstrates that $n f_{S} A$ and $n f_{s} B$ are probably upregulated members of the multiple antibiotic resistance regulon marR $A B$ (Barbosa and Levy, 2000), and that $n f s A$ is a member of the closely related oxidative stress response regulon soxRS (Liochev et al., 1999; Paterson et al., 2002). Here, the inclusion of $n f s B$ as part of these protective responses is investigated by assaying the activity of $\mathrm{Nfs} B$ after induction of each regulon.

In this chapter the results pertaining to $n f s B$ will be the primary focus. Characterization of the $n f s A$ gene in the nitrofuran resistant mutants was accomplished by Peter Koziarz (Koziarz, 2000), and his contribution is noted and included whenever necessary for clarification. J. Veall isolated the furazolidone resistant mutants as part of a fourth year honours project. Contributions to this work were also made by B. Hoecher (directed studies project), N. Sandhu and P. Kumar (technical assistance). A large part of the work detailed in this chapter was published as "Oxygen-insensitive nitroreductases: analysis of the roles of $n f_{s} A$ and $n f_{s} B$ in development of resistance to 5-nitrofuran derivatives in Escherichia coli" in the Journal of Bacteriology 180:5529-5539 (Whiteway et al., 1998). 


\subsection{Materials and Methods}

\subsubsection{Bacterial strains, plasmids and growth conditions}

E. coli K-12 strains AB1157, NFR402, NFR502, and SIL41 were provided by Dr. D. Bryant, McMaster University. AB1157 [F- thr-1 leu-6 thi-1 supE44 lacY1 galK2 ara$14 x y l-5$ mtl-1 proA2 his-4 argE3 str-31 tsx-33 $\lambda^{-}$sup-37] (Bachmann, 1972) is the parent strain of the nitroreductase mutants NFR402, and NFR502 (McCalla et al., 1978). Strain SIL41 was derived from a cross between AB1157 and an HfrH strain lacking NfsA and NfsB activity (Bryant et al., 1981). DH5 $\alpha$ [supE44 lacU169 (ф80lacZAM15) hsdR17 recAl endA1 gyrA96 thi-1 relA1] is a standard laboratory strain used for most plasmid manipulations. INV $\alpha \mathrm{F}^{\prime}\left[\mathrm{F}^{\prime}\right.$ endA1 recA1 hsdR17 $\left(\mathrm{r}_{\mathrm{k}}{ }^{-}, \mathrm{m}_{\mathrm{k}}{ }^{+}\right)$supE44 thi-1 gyrA96 relA1 $\phi 80$ lacZ $\Delta \mathrm{M} 15 \Delta\left(\right.$ lacZYA-argF)U169 $\left.\lambda^{-}\right]$was included in the Original TA Cloning ${ }^{\circledR}$ Kit (Invitrogen Corp., San Diego CA). The vectors used in this study were pUC118 (Viera and Messing, 1982), pKK232-8 from Pharmacia Biotech Inc. (Brosius, 1984), and $\mathrm{pCR}^{\circledR} 2.1$ from the Original TA Cloning ${ }^{\circledR}$ Kit. Bacterial strains were routinely grown at $37^{\circ} \mathrm{C}$ in Luria-Bertani (LB) medium, or on LBA (LB medium with $1.5 \%$ agar), obtained as a premixed powder from Canadian Life Technologies Inc. (Burlington, Ontario). When appropriate, media was supplemented with antibiotics in the following concentrations: $100 \mu \mathrm{g} / \mathrm{ml}$ ampicillin, $30 \mu \mathrm{g} / \mathrm{ml}$ chloramphenicol and $50 \mu \mathrm{g} / \mathrm{ml}$ kanamycin. Nitrofurazone (5-nitro-2-furaldehyde semicarbazone) and furazolidone (3(5-nitrofurfurylideneamino)-2-oxazolidinone) were kind gifts from Dr. D. Bryant, McMaster University, Hamilton; stock solutions were prepared in dimethylsulfoxide (DMSO). 


\subsubsection{Isolation of furazolidone resistant strains.}

Furazolidone resistant mutants were obtained as described previously using furazolidone instead of nitrofurazone (McCalla et al., 1975b). Briefly, furazolidone gradient plates were prepared as previously described (Szybalski, and Bryson, 1952) by applying a $10 \mathrm{ml}$ layer of furazolidone-containing LBA upon a slanted $10 \mathrm{ml}$ layer of LBA; twenty-four hours was allowed for diffusion of the drug prior to plating. Multiple cultures were grown to exponential growth phase and spread on the furazolidone gradient plates $(0-1.25 \mu \mathrm{g} / \mathrm{mL})$ which were incubated overnight. Several colonies were selected (first step mutants), subcultured and grown on gradient plates containing higher concentrations of the $\operatorname{drug}(0-2.5 \mu \mathrm{g} / \mathrm{mL}$ or $0-4 \mu \mathrm{g} / \mathrm{mL})$. Isolated colonies on these plates represented second step mutants.

\subsubsection{Determination of nitrofurazone sensitivity}

Overnight cultures of each mutant strain were streaked with a sterile cotton swab onto a gradient plate containing $0-50 \mu \mathrm{g} / \mathrm{mL}$ nitrofurazone. This provided a standard measure of resistance and allowed the new mutants to be compared with previously isolated strains (McCalla et al., 1975b). Absolute concentrations of nitrofurazone tolerance were determined as described by McCalla's group (McCalla et al., 1975b): 10 $\mu l$ of overnight cultures were inoculated in a grid pattern onto plates containing 5-45 $\mu \mathrm{g} / \mathrm{mL}$ concentrations of nitrofurazone.

\subsubsection{Preparation of clarified cellular extracts.}

Overnight cultures $(6 \mathrm{ml})$ were harvested by centrifugation; the cells were washed and resuspended in $0.25 \mathrm{ml} 50 \mathrm{mM}$ Tris- $\mathrm{HCl}(\mathrm{pH} 7.0)$. The suspensions were chilled on 
ice, sonically treated with a Kontes Micro-Ultrasonic Cell Disruptor (power setting

3.5) for two periods of $30 \mathrm{sec}$ each, and centrifuged at $14,000 \times \mathrm{g}, 4^{\circ} \mathrm{C}$ for 30 minutes.

The extracts typically contained $5-10 \mathrm{mg}$ of protein per $\mathrm{mL}$.

\subsubsection{Determination of protein concentration.}

Protein concentration was determined as described by the Bio-Rad Protein Assay (Richmond, California) using a bovine serum albumin standard curve.

\subsubsection{Nitrofurazone reductase assay.}

Nitrofurazone reductase activity was assayed by measuring the decrease in absorbance of nitrofurazone at $400 \mathrm{~nm}$ (molar extinction coefficient $12,960 \mathrm{M}^{-1} \mathrm{~cm}^{-1}$ ) at $22^{\circ} \mathrm{C}$ on a Pharmacia Biotech Ultrospec 3000 UV/Visible spectrophotometer. The reaction mixture $(1 \mathrm{ml})$ contained $50 \mathrm{mM}$ Tris- $\mathrm{HCl}(\mathrm{pH} 7.0), 0.1 \mathrm{mM}$ nitrofurazone and 0.1 mM NADPH or NADH.

\subsubsection{Manipulation of DNA.}

Genomic DNA was isolated from $4 \mathrm{ml}$ overnight cultures as described by Rodriguez and Tait (1989) with the exception that the precipitated DNA was centrifuged at $12,000 \times \mathrm{g}, 4^{\circ} \mathrm{C}$ for $10 \mathrm{~min}$., and the pellet was washed with $70 \%$ ethanol, dried and resuspended in $100 \mu \mathrm{l}$ TE. Plasmid DNA was isolated by the boiling miniprep method described by Holmes and Quiggley (1981) as follows. Cultures (5 ml) were centrifuged in a bench top centrifuge for 5 minutes at full speed ( $3000 \mathrm{rpm})$ and the pellet was resuspended in $400 \mu \mathrm{l}$ of STET buffer ( $8 \%$ sucrose, $5 \%$ TritonX-100, $50 \mathrm{mM}$ EDTA, 50 $\mathrm{mM}$ Tris, $\mathrm{pH} 8.0)$. Lysozyme $(0.5 \mathrm{mg} / \mathrm{ml}$ in $0.25 \mathrm{M}$ EDTA, $\mathrm{pH} 8.0)$ was added and the suspension was immediately boiled for 40 seconds with subsequent centrifugation for 5 
min at $4^{\circ} \mathrm{C}$ in an angle rotor Eppendorf centrifuge. The gelatinous pellet was removed with a toothpick, and the DNA was precipitated with 10M ammonium acetate and $95 \%$ ethanol. The pellet was washed with $70 \%$ ethanol, dried and resuspended in $20 \mu \mathrm{l}$ of TE (10mM Tris- $\mathrm{HCl}, 1 \mathrm{mM}$ EDTA, $\mathrm{pH} 8.0)$. If further purification was necessary, it was accomplished using a GENECLEAN ${ }^{\circledR}$ Kit (Bio 101, Inc.). Standard procedures were used for molecular cloning.

\subsubsection{Amplification of the $n f B$ gene.}

Amplification of the $n f s B$ gene (Figure 2.1) from genomic DNA was accomplished by PCR using the Expand ${ }^{\mathrm{TM}}$ High Fidelity PCR System (Boehringer Mannheim Canada). Prior to amplification, genomic DNA was digested with HindIII (New England Biolabs). Reaction mixtures $(100 \mu \mathrm{l})$ included the Expand ${ }^{\mathrm{TM}}$ High Fidelity buffer with $15 \mathrm{mM} \mathrm{MgCl}, 0.2 \mathrm{mM}$ of each dNTP, $300 \mathrm{nM}$ of appropriate primers (Table 2.1), 1.7 units of Expand ${ }^{\mathrm{TM}}$ High Fidelity enzyme mix, and 20-750 ng of template DNA. Amplification was performed through 30 repetitions of a [denaturation, $15 \mathrm{sec}$ at $94^{\circ} \mathrm{C}$; annealing $30 \mathrm{sec}$ at $55^{\circ} \mathrm{C}$; elongation, $1 \mathrm{~min}$ at $72^{\circ} \mathrm{C}$ ] cycle that included a $4 \mathrm{~min}$ preincubation at $94^{\circ} \mathrm{C}$ and a $7 \mathrm{~min}$ postincubation at $72^{\circ} \mathrm{C}$. Prior to further analysis the products were purified using a High Pure PCR Product Purification Kit (Boehringer Mannheim Canada). Amplification products and restriction endonuclease analyses were analyzed using agarose gel electrophoresis (1\%) in the presence of ethidium bromide.

\subsubsection{Cloning of $n f s B$.}

Cloning of amplified $n f s B$ into $\mathrm{pCR}^{\circledR} 2.1$ was carried out according to the Original TA Cloning ${ }^{\circledR}$ Kit instructions with the following changes. The Expand ${ }^{\mathrm{TM}}$ High Fidelity 
Figure 2.1 Nucleotide sequence of the PCR-generated fragment containing the $n f s B$ gene from $E$. coli $\mathrm{AB} 1157$ with putative $-35,-10$, ribosomal binding site and transcription terminator indicated. NFR502 and SIL41 refer to the mutants isolated by McCalla and coworkers (McCalla et al., 1978). Mutations for the 50 mutants isolated in this study are indicated. Altered bases and amino acids are underlined in the wild-type sequence, with the substituted bases shown above the sequence. IS elements are represented by boxes above the sequence, and the insertion point is indicated with an arrow. Deletions and insertions are indicated below the sequence. Horizontal arrows represent the PCR primers, and lowercase letters indicate the synthetic endonuclease sites added onto the PCR primers. S.D., Shine-Dalgarno sequence. 


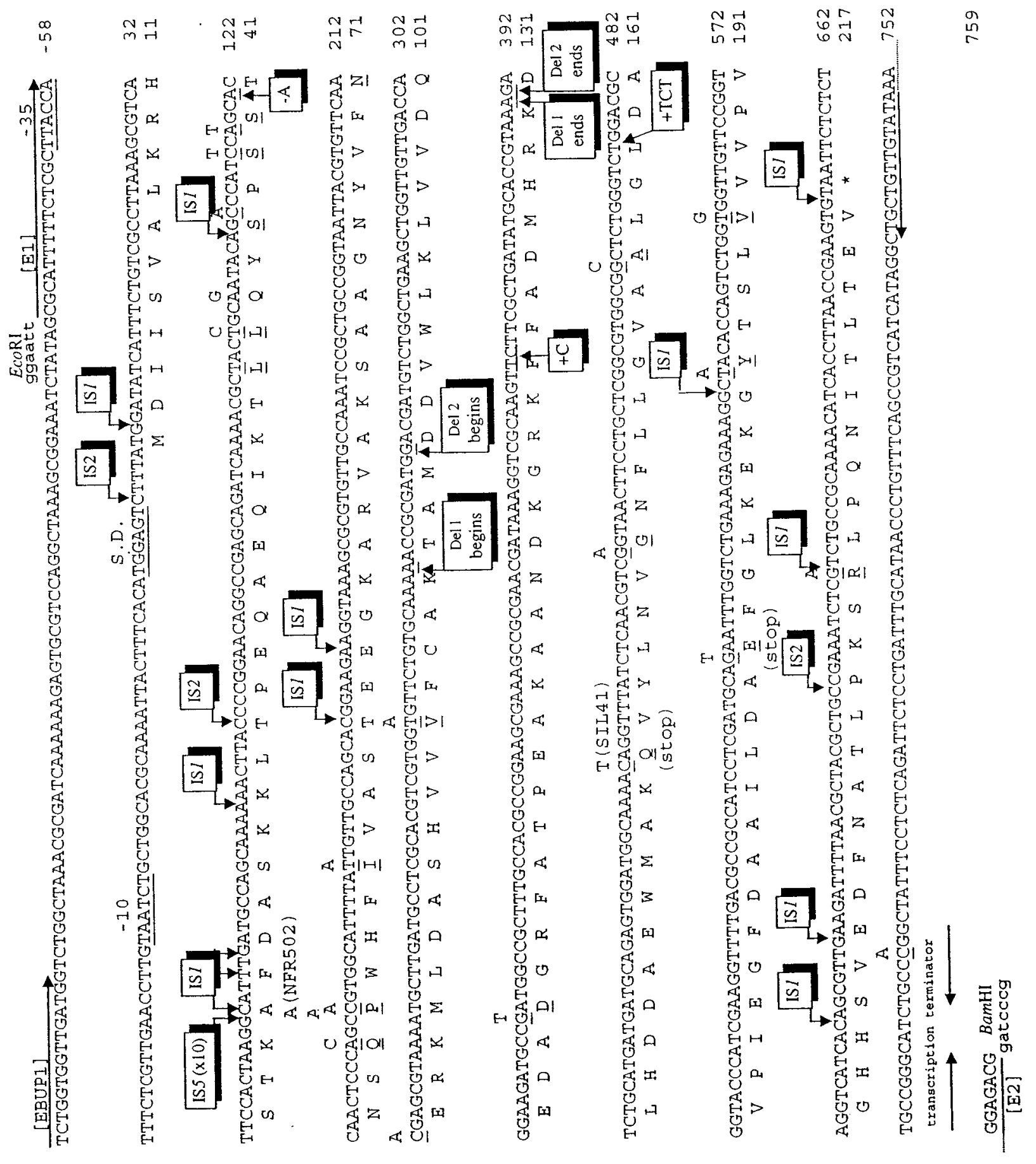


Table 2.1 Primers used in this study. Refer to the $n f s B$ sequence in Figure 2.1 for primer locations.

\begin{tabular}{llc}
\hline Primer & Sequence 5' $^{\prime} 3^{\text {,a }}$ & Strand $^{\text {b }}$ \\
\hline E1 & Ggaattc $^{-86}$ GCATTTTTCTCGCTTACCA & $\mathrm{nt}$ \\
E2 & Cgggatc $^{+108}$ cGTCTCCTTTATACAACAGCA & $\mathrm{t}$ \\
EBup1 & ${ }^{-90}$ TCTGGTGGTTGATGGTCTGG & $\mathrm{nt}$ \\
ET2 & ${ }^{216}$ GCGTAAAATGCTTGATG & $\mathrm{nt}$ \\
ET3 & ${ }^{499}$ TTTGACGCCGCCATCCT & $\mathrm{nt}$ \\
EN2 & ${ }^{592}$ GGATGGCGGCGTCAAAA & $\mathrm{t}$ \\
EN3 & ${ }^{312}$ GGCATCAAGCATTTTAC & $\mathrm{t}$ \\
EN4 & ${ }^{50}$ TCAAATGCCTTAGTGGAATG & $\mathrm{t}$ \\
\hline
\end{tabular}

Notes: ${ }^{a}$ Superscript number indicates base number in $n f s B$. A minus or plus sign refers to bases upstream and downstream, respectively, of the coding (i.e. translatable) region of $n f s B$. Lowercase letters indicate restriction enzyme recognition sites.

$\mathrm{b}_{\mathrm{nt}}=$ non-transcribed strand (i.e. coding); $\mathrm{t}=$ transcribed strand (i.e. noncoding) 
PCR System was used to amplify the genes from AB1157 followed by a 10 minute incubation at $72^{\circ} \mathrm{C}$ with 1 Unit of Taq polymerase (Canadian Life Technologies Inc.) to add a 3' A-overhang. The correct sequence of a successful clone, pTAB (Figure 2.2), was verified by sequencing with universal primers for pUC vectors as well as with specific internal primers for $n f s B$. The similarly constructed clone containing the $n f s A$ gene, pTAA (Figure 2.2) was obtained from P. Koziarz. The $n f s B$ PCR fragment was inserted into the EcoRI and BamHI sites of pUC118 to create pJAB (Figure 2.3a). The primers used for amplification of the $850 \mathrm{bp} n f s B$ fragment contained these 5 ' restriction endonuclease recognition sites to facilitate cloning. pJAC (Figure 2.3b) was constructed by subcloning the $469 \mathrm{bp} E c o \mathrm{RI}($ filled)-BglII fragment of pJAB into the $S m a \mathrm{I}$ and $B a m \mathrm{HI}$ sites of pKK232-8. The 3' termini of EcoRI were filled in by adding $2 \mu \mathrm{l}$ each of dATP and dTTP $1 \mathrm{mM}$ stocks, and 2 units of Klenow polymerase to the pJAB heat inactivated EcoRI digest.

\subsubsection{Sequencing of clones and PCR products.}

Sequencing of the PCR products from each mutant was achieved using the Sequenase $^{\mathrm{TM}}$ v.2.0 T7 DNA Sequencing Kit and $\left[\alpha_{-}^{35} \mathrm{~S}\right] \mathrm{dATP}$ (Amersham Canada Ltd.).

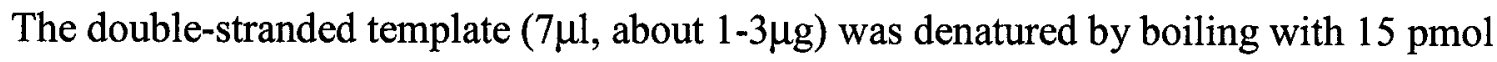
$(1 \mu 1)$ of primer and quick-chilled on ice. Chilled Sequenase reaction buffer $(2 \mu \mathrm{l}), 0.1 \mathrm{M}$ dithiothreotol $(1 \mu \mathrm{l}), 1: 5$ dilution of dGTP labeling mix $(2 \mu 1)$ and $0.5 \mu 1(5 \mu \mathrm{Ci})$ of $[\alpha-$ $\left.{ }^{35} \mathrm{~S}\right] \mathrm{dATP}$ were added to the reaction. Immediately prior to the termination reaction, $2 \mu \mathrm{l}$ of diluted (1:7) Sequenase enzyme was added. For termination, $3.2 \mu 1$ of the reaction mixture was added to $2.5 \mu \mathrm{l}$ of each ddNTP, incubated at $37^{\circ} \mathrm{C}$ for $5 \mathrm{~min}$., and stopped by 


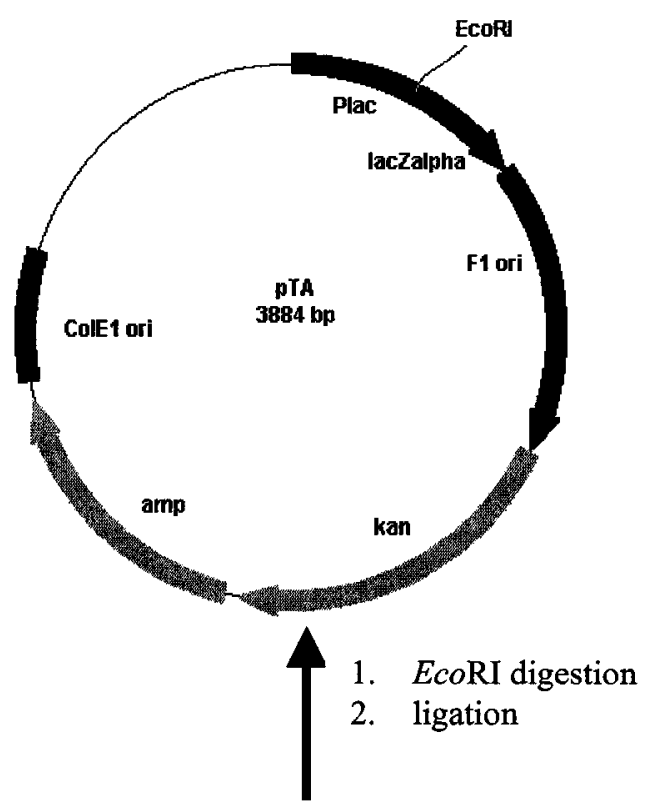

pCR2.1 3900bp
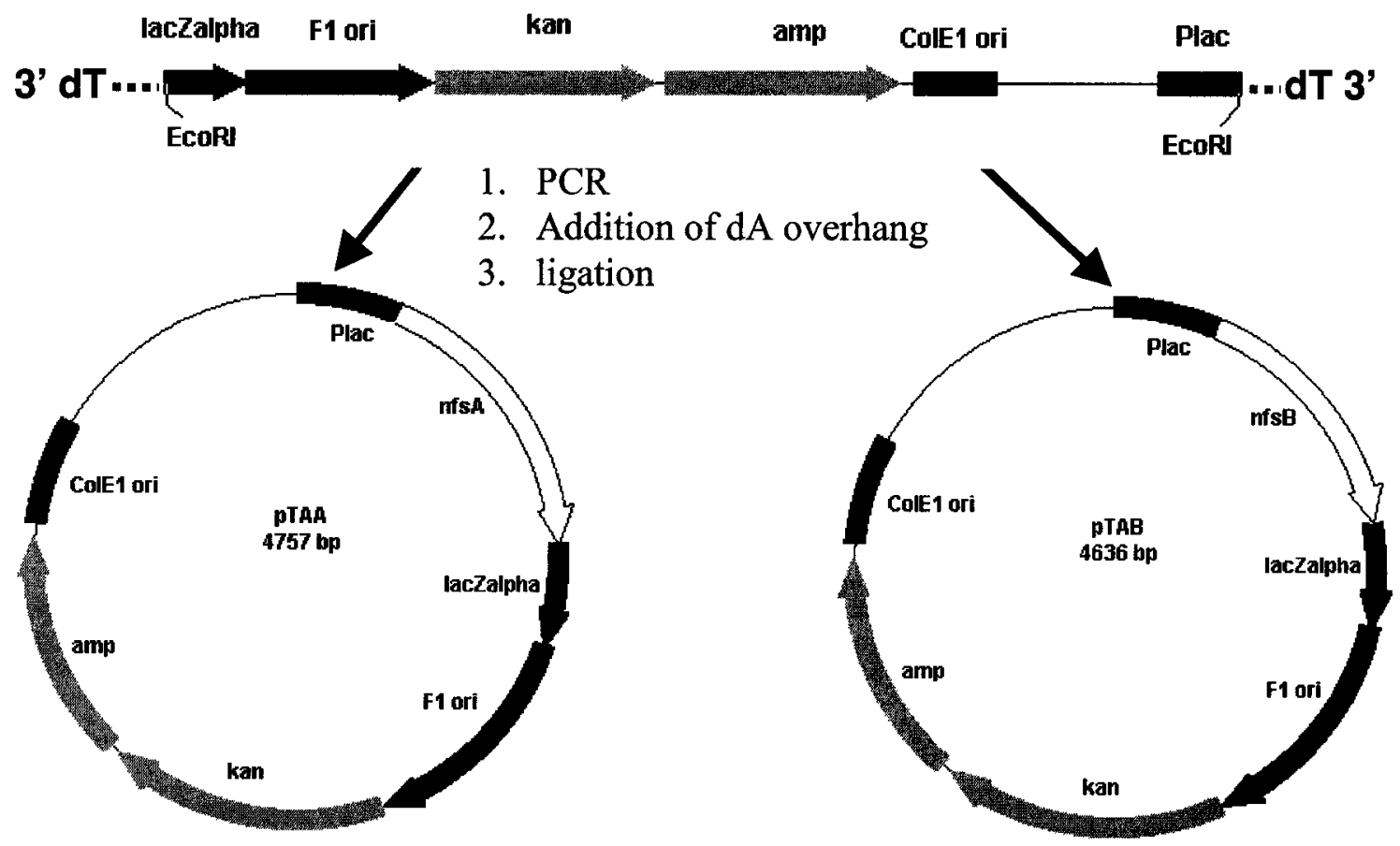

Figure 2.2 The linear $\mathrm{pCR}^{\circledR} 2.1$ vector (Invitrogen) is shown in the centre and the clones pTAA (Koziarz, 2000) and pTAB containing PCR amplified $n f s A$ and $n f s B$, respectively, are indicated below. A circular vector (pTA) was derived from the linear form by digestion with EcoRI directly followed by ligation. 
a.

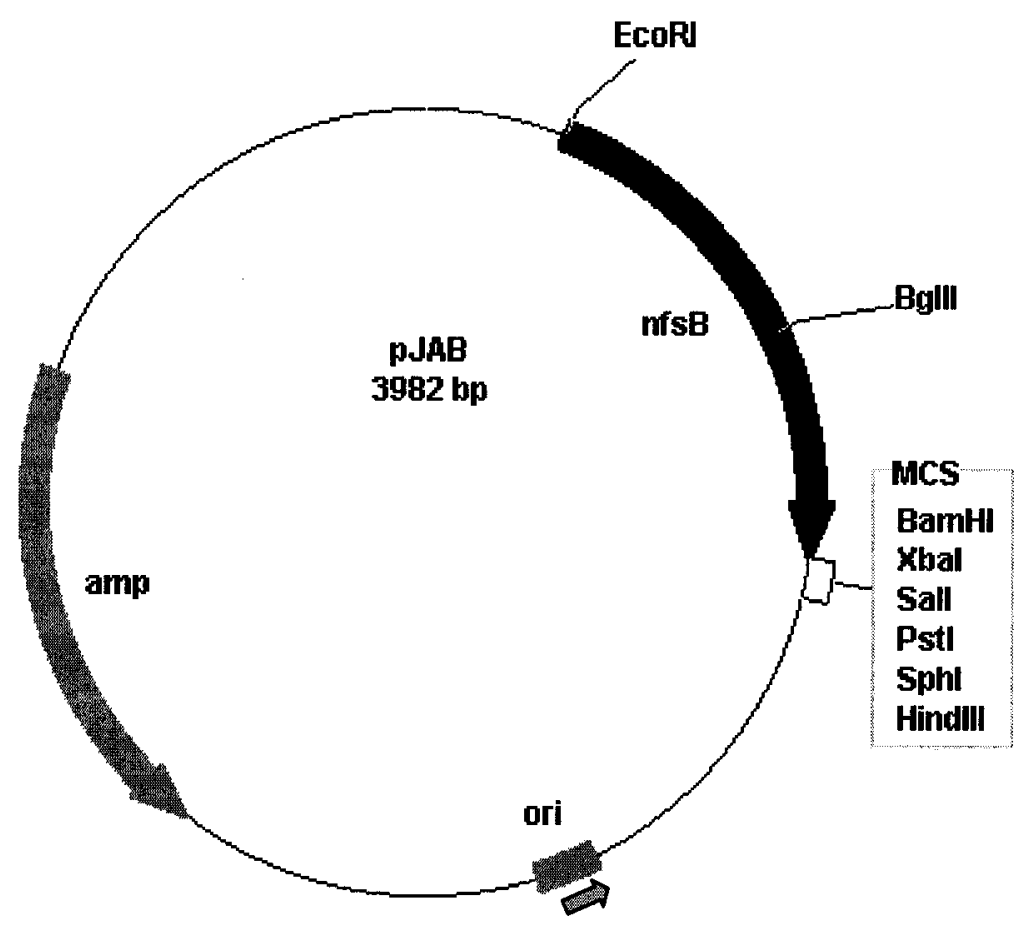

b.

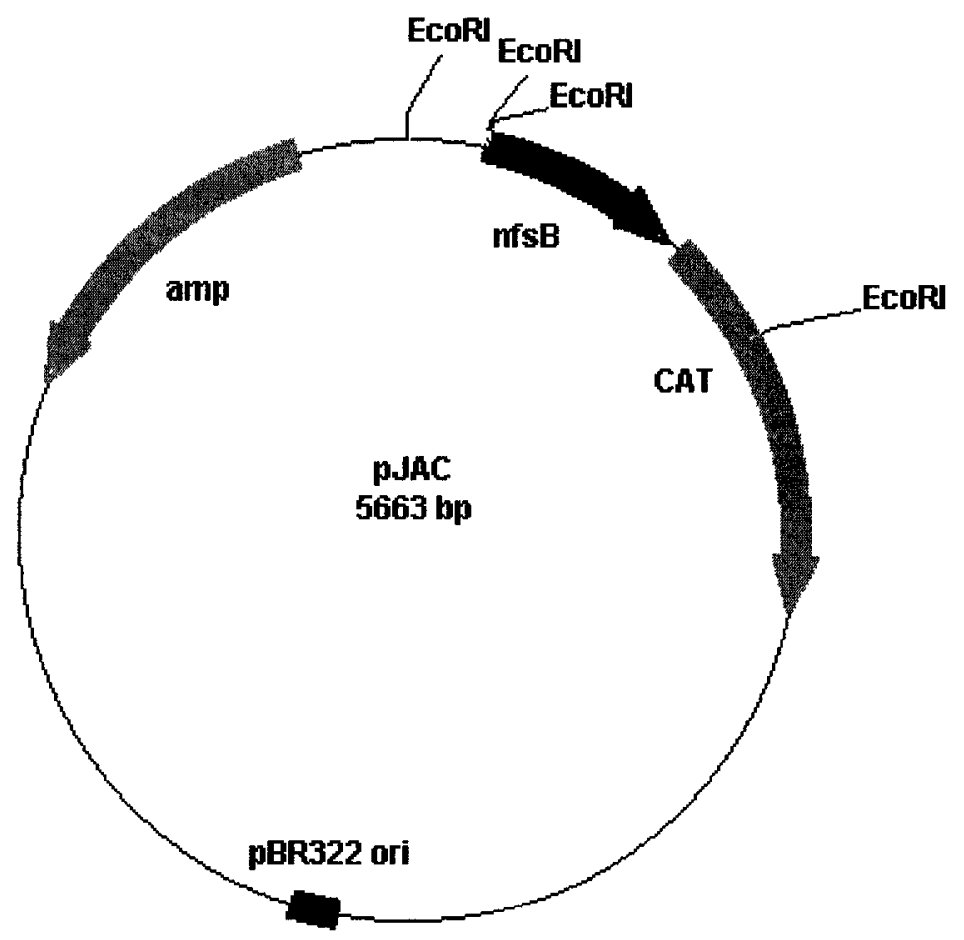

Figure 2.3 Plasmid clones of $n f s B$. (a) pJAB: PCR-amplified $n f s B$ inserted in pUC118. (b) pJAC: Truncated $n f s B$, lacking the putative transcription terminator, inserted into the pKK232-2 vector containing the CAT gene. This construct was used to test for the presence of the $n f s B$ promoter in the amplified sequence (approximately 140 bp upstream of the initiation codon). 
the addition of $4 \mu \mathrm{l}$ STOP solution ( $95 \%$ formamide, $20 \mathrm{mM}$ EDTA, $0.05 \%$ bromophenol blue, $0.05 \%$ xylene cylanol). The reactions were preheated at $80^{\circ} \mathrm{C}$ for 2 minutes, and analyzed on an $8 \%$ acrylamide/bisacrylamide gel.

IS elements were identified by DNA sequencing the gene/IS element endpoints followed by a nonredundant similarity search of sequence databases and the Escherichia coli Databank (Nara Institute of Science and Technology, Japan) with BLASTX (Altschul et al., 1990).

\subsubsection{Nitrofurazone sensitivity assay.}

Overnight cultures $(100 \mu \mathrm{l})$ of each strain were added to $4 \mathrm{ml}$ of LB top agar (LB medium solidified with $0.7 \%$ agar) to create a lawn of bacteria on which $10 \mu$ l of various concentrations of nitrofurazone, in DMSO, were applied in triplicate to paper disks arranged on the lawn. Overnight incubation at $37^{\circ} \mathrm{C}$ yielded cleared zones in the bacterial lawn, the diameter of which was measured.

\subsubsection{Nitrofurazone and furazolidone susceptibility assay.}

LB (4 ml) was inoculated with a few colonies and grown at $37^{\circ} \mathrm{C}$ until $\mathrm{A}_{600} \approx 0.3$. Two microtubes with $1 \mathrm{ml}$ of the suspension were centrifuged at 14,000xg, $5 \mathrm{~min}$., and the pellets were washed with $0.1 \mathrm{M}$ Tris- $\mathrm{HCl}(\mathrm{pH} 7.6)$. One pellet was resuspended in 0.1 $\mathrm{ml}$ of $0.1 \mathrm{M}$ Tris- $\mathrm{HCl}(\mathrm{pH} 7.6)$, the other in $0.1 \mathrm{ml}$ of $0.1 \mathrm{M}$ Tris- $\mathrm{HCl}(\mathrm{pH} \mathrm{7.6)} \mathrm{containing}$ $0.2 \mathrm{mM}$ EDTA. After incubation at $37^{\circ} \mathrm{C}$ for $2 \mathrm{~min} ., 1 \mathrm{ml}$ of Mueller-Hinton broth (Beef infusion, Casamino acids, starch) (Difco) was added. A sterile cotton swab was used to inoculate the entire surface of Mueller-Hinton Agar plates, and allowed to dry for no more than 15 minutes. Susceptibility Test Disks (Difco) infused with nitrofurazone (75 
$\mu \mathrm{g})$ or furazolidone $(40 \mu \mathrm{g})$ were placed, in duplicate, on the plates. Overnight incubation at $37^{\circ} \mathrm{C}$ yielded cleared zones in the bacterial lawn, the diameter of which was measured.

\subsubsection{Chloramphenicol acetyltransferase assay.}

The specific activity of chloramphenicol acetyltransferase (CAT) was determined according to the previously described spectrophotometric assay (Rodriguez and Tait, 1983). This method measures free 5-thio-2-nitrobenzoate at $\lambda=412 \mathrm{~nm}$ which has a direct molar relationship to the acetylation of chloramphenicol in the presence of 5,5'dithiobis-2-nitrobenzoic acid and acetyl CoA (Shaw, 1975). Cell extracts were prepared from $3 \mathrm{ml}$ cultures grown to $\mathrm{A}_{450}=1.2$ in $\mathrm{LB}$ supplemented with $0.2 \%$ glucose. The harvested cells were washed with $1 \mathrm{ml}$ of TDTT buffer $(50 \mathrm{mM}$ Tris-HCl $\mathrm{pH} 7.8,30 \mu \mathrm{M}$ DL-dithiotheitol) and resuspended in $300 \mu$ TDTT buffer. The suspensions were chilled on ice, sonically treated with a Kontes Micro-Ultrasonic Cell Disruptor (power setting 3.5) for two periods of $30 \mathrm{sec}$ each, and centrifuged at $14,000 \times \mathrm{g}, 4^{\circ} \mathrm{C}$ for 15 minutes. The reaction mixture contained $1 \mu \mathrm{M}$ DTNB [5,5'-dithiobis-(2 nitrobenzoic acid) in $1 \mathrm{M}$ Tris- $\mathrm{HCl} \mathrm{pH} 7.8$ ], $0.1 \mathrm{mM}$ Acetyl-CoA and $\mathrm{ddH}_{2} 0$. The $0.588 \mathrm{ml}$ reaction mixture was incubated at $37^{\circ} \mathrm{C}$ for $2 \mathrm{~min}$. and the $\mathrm{A}_{412}$ was adjusted to 0.0 . Crude extract $(20 \mu \mathrm{l})$ was added, mixed well and equilibrated for 2 minutes. Background acetylation was measured for 2 min until the slope was constant. The reaction was initiated with the addition of 12 $\mu \mathrm{l} 5 \mathrm{mM}$ chloramphenicol, mixed well, and $\mathrm{A}_{412}$ was recorded for 4 minutes. CAT activity was calculated using the molar extinction coefficient $\left(0.0136 \mathrm{nM}^{-1} \mathrm{~cm}^{-1}\right)$ and subtracting the background rate of acetylation. 


\subsubsection{Protein sequence analysis.}

A similarity search of protein sequence databases was conducted using the BLAST server program (Altschul, 1990) with default parameters. Protein alignments were performed by the ClustalV program (Higgins and Sharp, 1989) using default parameters.

\subsubsection{Induction of nitroreductase by paraquat and salicylate.}

Overnight cultures of AB1157, SIL41, JVQ1 and JVQ2 were diluted 20-fold into fresh $\mathrm{LB}$ medium and incubated at $37^{\circ} \mathrm{C}$ for 1 hour. Paraquat $(0.2 \mathrm{mM})$ and sodium salicylate $(5 \mathrm{mM})$ were added to the appropriate cultures and incubation was continued for one hour. Clarified cellular extracts were prepared and assayed for protein concentration and nitroreductase activity. Two independent extracts were made and each was assayed in duplicate. The confidence interval (95\%) of the mean nitroreductase activity in each strain was calculated in Microsoft Excel. 


\section{$\underline{2.3 \text { Results }}$}

\subsubsection{Isolation of mutant strains.}

Putative nitroreductase mutants derived from $\mathrm{AB} 1157$ were identified on the basis of their resistance to furazolidone after stepwise selection on gradient plates. Mutant resistance to nitrofurazone, as measured by growth on a gradient plate, was used to standardize the level of nitrofuran resistance exhibited by these mutants, and allow comparison to mutants selected in previous studies (Bryant et al., 1981; McCalla et al., 1978). The first and second selection steps yielded mutants that were resistant to nitrofurazone concentrations of approximately $15 \mu \mathrm{g} / \mathrm{ml}$ and $35 \mu \mathrm{g} / \mathrm{ml}$, respectively. Resistance occurred incrementally: there were no instances in which a single selection step yielded a mutant that exhibited resistance to the elevated concentrations of furazolidone characteristic of second step mutants. Subsequent selection steps did yield third step mutants that exhibited resistance to still higher concentrations of furazolidone

( $>4 \mu \mathrm{g} / \mathrm{ml}$ ) though they maintained characteristic second step nitrofurazone resistance. The focus of this study is primarily on the genetic analysis of first and second step mutants.

\subsubsection{Genetic analysis.}

DNA sequence analysis was performed on the PCR generated amplified products of $n f s A$ (Koziarz, 2000) and $n f s B$ obtained from all of the furazolidone resistant mutants isolated in this study, as well as from the nitrofurazone resistant mutants NFR402, NFR502 and SIL41 obtained previously by other workers (McCalla et al., 1978). Following sequence analysis, some mutants derived through a single experiment had 
identical mutations. To ensure that all mutations were of independent origin, only one identical mutant from each experiment was considered in our analysis. The strain designations and mutations are shown in Table 2.2.

All eighteen of the first step nitrofuran resistant mutants isolated in this study and the previously isolated first step mutant NFR402 contain a mutation in the $n f s A$ gene (Tables 2.2 and 2.3). No mutations in the $n f s B$ gene were observed in any of the first step mutants, while all but three of the 53 second step mutants revealed a mutation in the $n f s B$ gene (Tables 2.2 and 2.3). SIL41, derived from a genetic cross between an Hfr $n f s A n f s B$ double mutant and AB1157, exhibits wild-type levels of resistance to nitrofurazone. However, SIL41 can be mutated to high (two step) levels of nitrofuran resistance through a single mutational step. SIL41 has a wild-type $n f s A$ gene, and contains an amber mutation, Gln-124 (CAG) to amber (UAG), at position $\mathrm{C}^{424}$ in $n f s B$ (Figure 2.1).

The second step mutants isolated in this study were confirmed to have only the parental mutation in $n f s A$. Only single mutations were found in the $n f_{s} B$ gene of second step mutants (refer to Figure 2.1). Eighteen of the 53 second step mutants have a base substitution in $n f s B$ : one results in an ochre mutation, 17 are missense mutations. Of the 17 missense mutations, nine effected changes in a 17 amino acid sequence, from Leu-33 to Ile-49, of the NfsB protein. One mutant, JVZ2, has a three base insert (TCT) at position $\mathrm{T}^{477}$ that results in the addition of a leucine residue between Leu-59 and Asp- 60 . The two mutants JVAE6 and JVI3 contain frameshift mutations in $n f s B$ resulting from the insertion of a cytosine at position $\mathrm{C}^{369}$ and the deletion of $\mathrm{A}^{121}$, events causing premature termination of translation after two and 53 amino acids, respectively. In two 


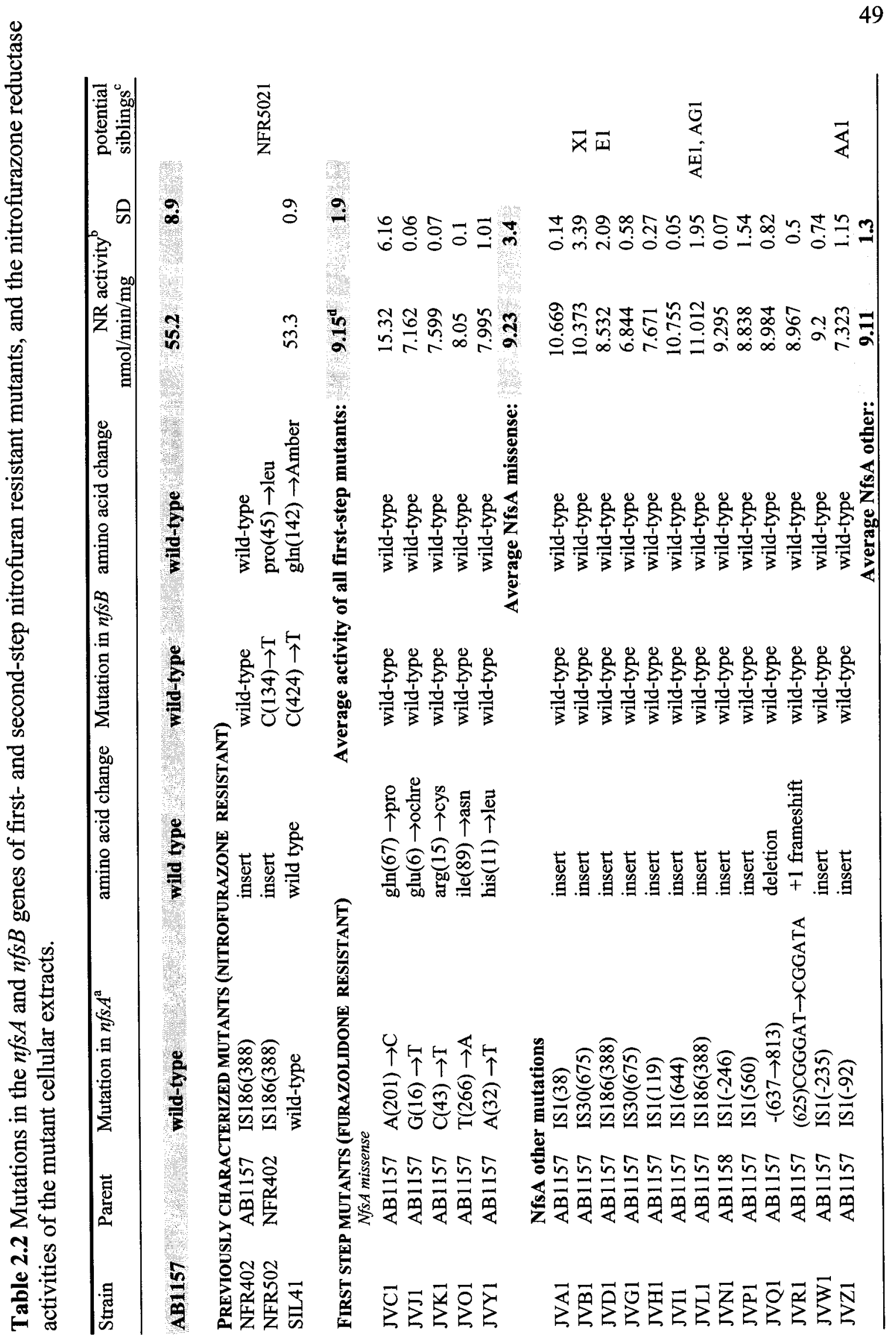




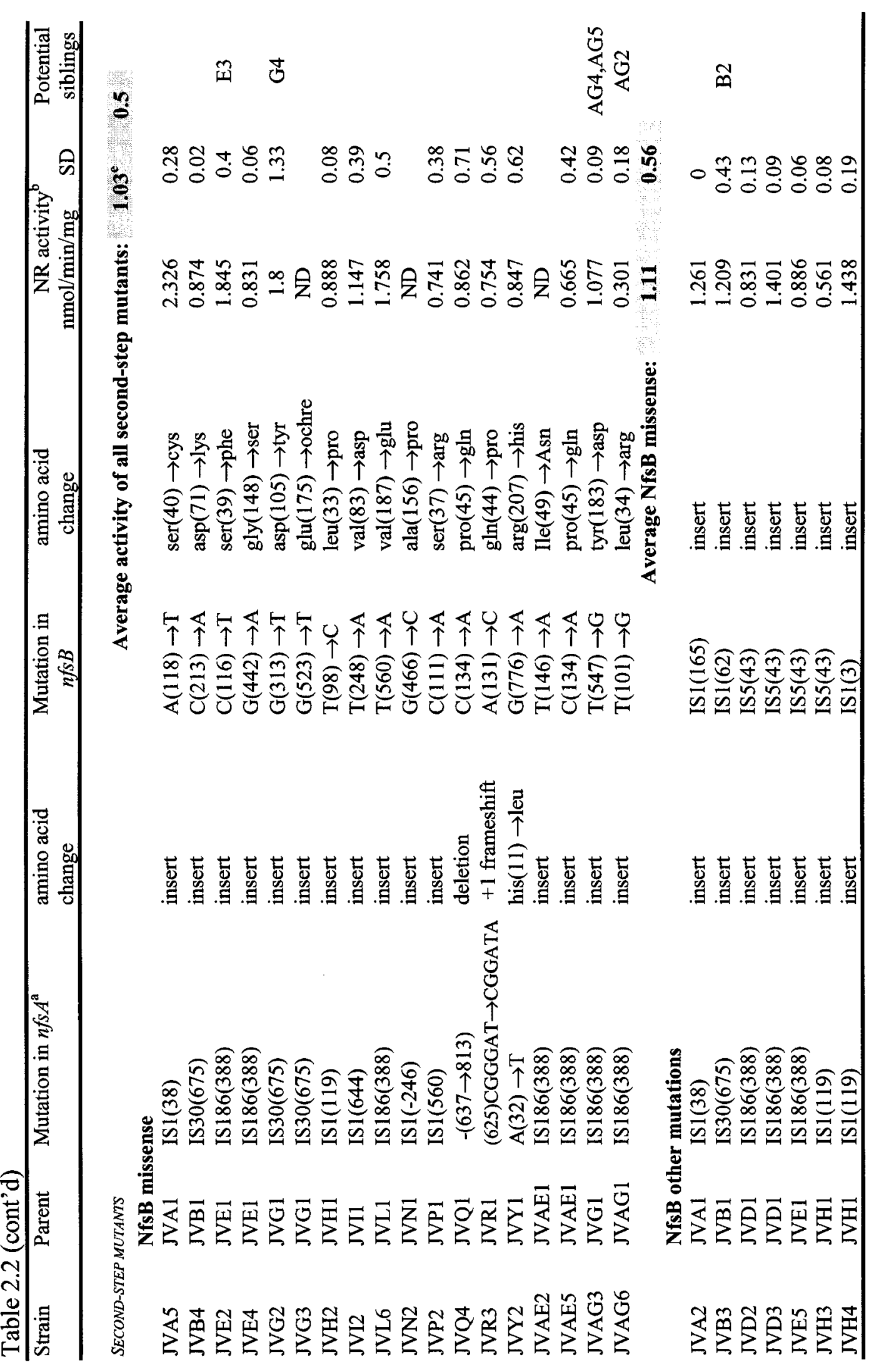




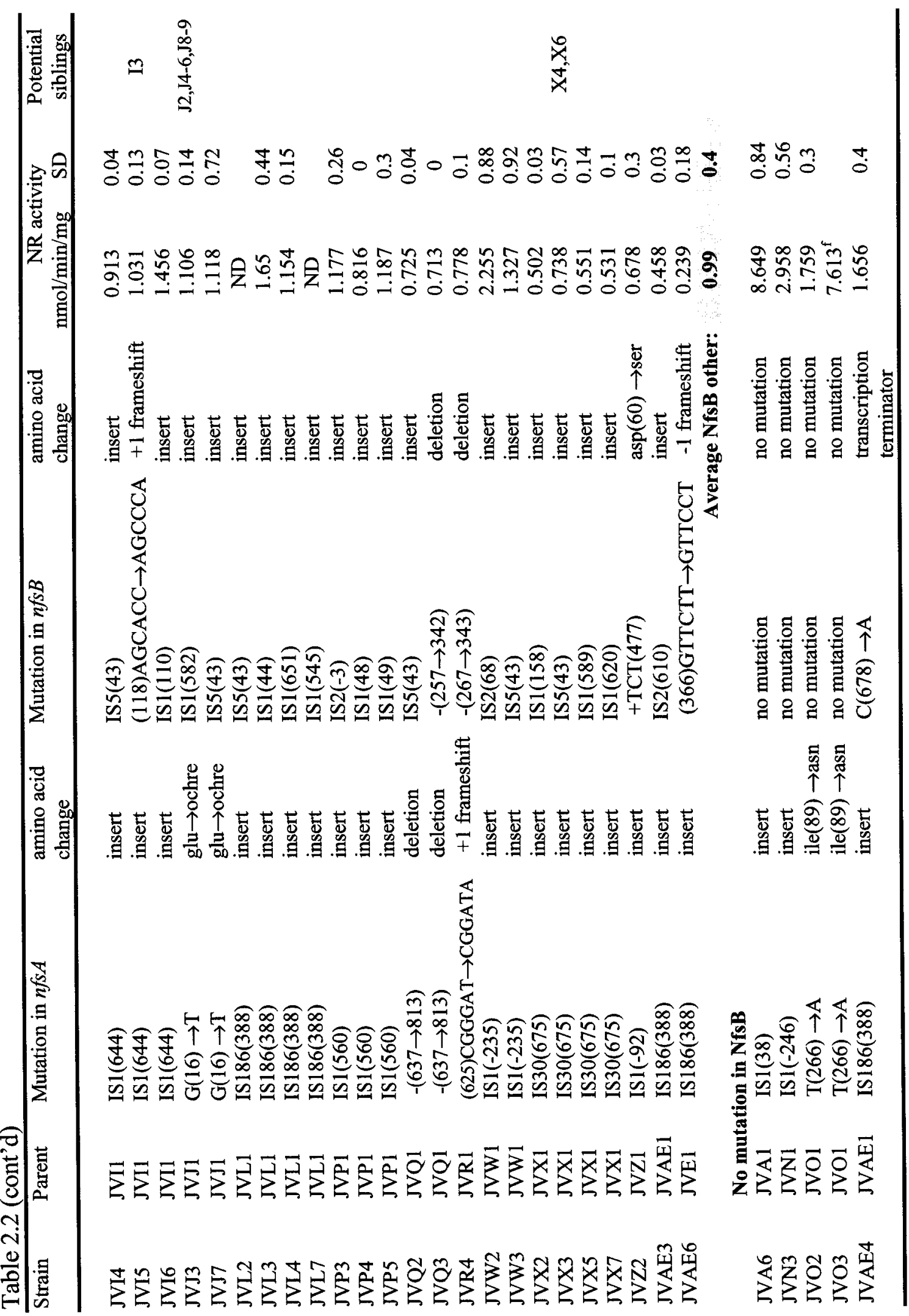




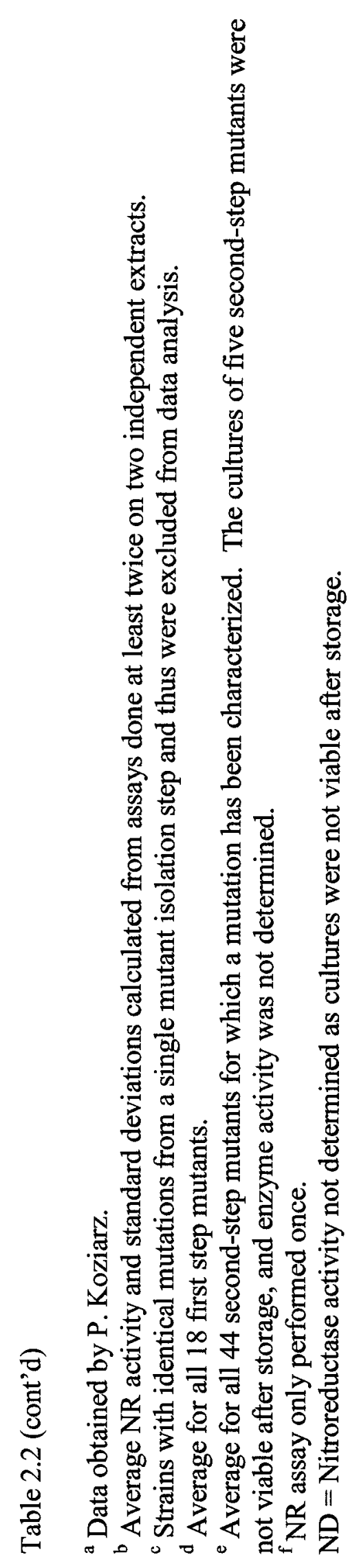


Table 2.3 Mutation distribution in $n f_{s} A$ and $n f s B$ of nitrofuran resistant mutants.

\begin{tabular}{|c|c|c|}
\hline & $\begin{array}{c}n f s A \\
{\text { (of } 1^{\text {st }} \text { step mutants) }}^{\text {a }}\end{array}$ & $\begin{array}{c}n f s B \\
\text { (of } 2^{\text {nd }} \text { step mutants) }\end{array}$ \\
\hline \multicolumn{3}{|l|}{ Base substitutions } \\
\hline Total & 5 & $20^{d}$ \\
\hline $\mathrm{G}: \mathrm{C} \rightarrow \mathrm{T}: \mathrm{A}$ & 1 & 6 \\
\hline $\mathrm{G}: \mathrm{C} \rightarrow \mathrm{C}: \mathrm{G}$ & 0 & 1 \\
\hline $\mathrm{A}: \mathrm{T} \rightarrow \mathrm{T}: \mathrm{A}$ & 2 & 4 \\
\hline$A: T \rightarrow C: G$ & 1 & 3 \\
\hline $\mathrm{G}: \mathrm{C} \rightarrow \mathrm{A}: \mathrm{T}$ & 1 & $5^{\mathrm{d}}$ \\
\hline $\mathrm{A}: \mathrm{T} \rightarrow \mathrm{G}: \mathrm{C}$ & 0 & 1 \\
\hline Frameshifts and insertions & 1 & 3 \\
\hline Deletions & 1 (177 bp) & $2(\sim 80 \mathrm{bp})$ \\
\hline Insertion elements & 12 & 26 \\
\hline IS 1 & 7 & 13 \\
\hline IS 2 & 0 & 3 \\
\hline IS5 & 0 & 10 \\
\hline IS30 & 2 & 0 \\
\hline IS 186 & $3^{c}$ & 0 \\
\hline No mutation in gene & $\mathbf{0}$ & 4 \\
\hline Total \# of mutants & $18\left(19^{c}\right)$ & $53\left(55^{d}\right)$ \\
\hline
\end{tabular}

${ }^{a}$ Data obtained by P. Koziarz. The same mutations, and no others, are in $n f_{s} A$ of second step mutants derived from first step mutants.

${ }^{\mathbf{b}}$ The $n f_{s} B$ gene of first step mutants is wild-type.

'The mutant NFR402 isolated by McCalla et al. (1978) is included.

${ }^{\mathrm{d}}$ The mutants NFR502 and SIL41 isolated by McCalla et al. (1978) are included. 
mutants, JVQ3 and JVR4, nearly identical deletions of approximately $80 \mathrm{bp}$ are situated in the middle of the gene. The most frequent type of mutation identified in the $n f_{s} B$ gene of the furazolidone resistant mutants is IS element integration. Three IS elements, IS 1 , IS2, and IS 5 , account for $49 \%$ of the second step mutants isolated in this study. With the one exception of IS1 inserting near the Shine-Dalgarno sequence of JVP3, all IS elements integrated within the coding region of $n f s B$. IS elements demonstrated regional specificity for insertion in the regions proximate to either terminus of $n f s B$. This is particularly apparent in the first $165 \mathrm{bp}$ of the gene's coding region where IS element integration occurred in 19 mutants. IS 5 integrated at the identical sequence, after $\mathrm{G}^{43}$, in all ten mutants with this element; IS5 inserted in the identical orientation in each mutant with the exception of JVD2 in which it was reversed. In four second step mutants, no mutations were observed within the coding region of $n f s B$ although multiple sequencing reactions were carried out on both strands of the PCR products. Of these, one mutant, JVAE4, contained a transversion in the putative transcription terminator. The resistance of these mutants to a concentration of nitrofurazone $(45 \mu \mathrm{g} / \mathrm{ml})$ consistent with a second step mutant was confirmed. To ensure that there is not a mutation in or near the putative -35 region of $n f_{s} B$, another primer, EBUP1 (see Figure 2.1), was used to amplify and sequence this region. No mutations were found.

\subsubsection{Promoter activity of $\mathrm{nfsB}$.}

The wild-type sequence determined for the complete $n f s B$ gene of the three second step mutants JVA3, JVN3 and JVO2 suggested the possibility that the promoter 
region might be inactive. We confirmed the presence of a functional promoter in the PCR amplified product of $n f_{s} B$ by using the construct pJAC (Figure 2.3b). pJAC contains the promoterless chloramphenicol acetyltransferase (CAT) gene of plasmid pKK232-8 directly downstream from the truncated fragment of $n f_{s} B$, containing the putative promoter region, so that any expression of CAT, and hence resistance to chloramphenicol, was due to the promoter activity of $n f s B$. Growth of transformants derived from $\mathrm{pJAC}$ on chloramphenicol medium confirmed the presence of a functional $n f s B$ promoter. This construct enabled the measurement of promoter activity by determining the specific activity of CAT $\left(126 \mathrm{nmol} \mathrm{min}^{-1} \mathrm{mg}^{-1}\right)$. The specific activity of CAT in the negative control, containing only pKK232-8, was zero.

\subsubsection{Nitroreductase activity.}

Nitroreductase activities in cell extracts of AB1157 (wild-type), first step mutants and second step mutants were spectrophotometrically measured as the reduction of nitrofurazone using NADPH as a source of reducing equivalents. The average NADPHdependent nitroreductase activities of $\mathrm{AB} 1157$, first step mutants, and second step mutants were 55.2, 9.2 and $1.0 \mathrm{nmol} \mathrm{min}^{-1} \mathrm{mg}^{-1}$, respectively (Table 2.2). Three second step mutants have significantly greater nitroreductase activity than the average: JVA6 and JVN3, two of the three second step mutants that do not have a mutation in NfsB; and JVA5, which has a missense mutation (Ser-40 $\rightarrow$ Cys) in NfsB. Of particular interest is JVA6, which has nitroreductase activity $\left(8.4 \pm 0.8 \mathrm{nmol} \mathrm{min} \mathrm{mg}^{-1}\right)$ comparable to a first step mutant. JVN3 has intermediate nitroreductase activity $\left(3.0 \pm 0.56 \mathrm{nmol} \mathrm{min} \mathrm{mg}^{-1}\right)$ that is approximately three fold less and greater than first- and second- step mutants, 
respectively. The second step mutant, JVAE4, which has a mutation in the putative transcription terminator but not in the coding region of $n f s B$, has nitroreductase activity consistent with second step mutants. JVO2, the third mutant without a mutation in $n f s B$, has activity $\left(1.8 \pm 0.3 \mathrm{nmol} \mathrm{min} \mathrm{mg}^{-1}\right)$ that is within the activity range for second step mutants. Although JVO3 is a potential sibling of JVO2, the NR activity of JV03 (7.6 $\mathrm{nmol} \mathrm{min} \mathrm{mg}^{-1}$ ) is similar to a first-step mutant. SIL41 has nitroreductase levels (53.3 \pm $0.9 \mathrm{nmol} \mathrm{min}{ }^{-1} \mathrm{mg}^{-1}$ ) similar to the wild-type.

\subsubsection{Restoration of nitrofuran sensitivity by cloned $N f_{\mathrm{s} A} \mathrm{and} N f_{\mathrm{s} B}$.}

The influence of plasmid encoded NfsA and $\mathrm{NfsB}$ in vivo was evaluated for the wild-type $E$. coli strain $\mathrm{AB} 1157$, a first step mutant containing a 177 bp deletion in $n f s A$ (JVQ1), and a second step mutant derived from JVQ1 with IS5 integrated after position 43 of $n f s B$ (JVQ2), by comparing their levels of nitrofurazone sensitivity to those observed when the strains were transformed with pTAA $(n f s A)$ or pTAB $(n f s B)$ (Figure 2.2). Sensitivity was compared by measuring the diameters of cleared zones around a nitrofurazone-infused paper disk on a lawn of bacteria (Table 2.4). The greatest differences in sensitivity were noted for concentrations between $5 \mathrm{mg} / \mathrm{ml}$ and $10 \mathrm{mg} / \mathrm{ml}$ of nitrofurazone. Further increases in sensitivity were not observed at concentrations above $10 \mathrm{mg} / \mathrm{ml}$, and concentrations of $1 \mathrm{mg} / \mathrm{ml}$ or lower did not yield cleared zones at all.

Cleared zone diameters obtained with each strain are shown in Table 2.4. From this experiment, four observations were made. (1) AB1157, JVQ1, and JVQ2 each had a significantly different sensitivity to nitrofurazone; $A B 1157$ demonstrated the greatest 
Table 2.4 Diameters ( $\mathrm{mm}$ ) of cleared zones in a lawn of each strain with and without vector encoded NfsA (pTAA) and NfsB (pTAB) after the application of $5 \mathrm{mg} / \mathrm{ml}$ nitrofurazone.

\begin{tabular}{lccc}
\hline Strain & vector alone (pTA) & + pTAA & + pTAB \\
\hline AB1157 & $18.4(0.4)$ & $20.6(0.5)$ & $20.9(0.5)$ \\
JVQ1 & $15.5(0.5)$ & $18.8(1.3)$ & $18.9(0.7)$ \\
JVQ2 & $12.5(0.5)$ & $20(1)$ & $18.8(0.8)$ \\
\hline
\end{tabular}

Note: Standard deviations calculated from five determinations are indicated in parentheses. 
sensitivity, and JVQ2 the least. (2) Upon the introduction of either plasmid-encoded nitroreductase, an increase in sensitivity was observed over that exhibited by the strain transformed with vector alone. (3) Between strains, no significant difference between the sensitivity conferred by the $n f_{s} A$ - or the $n f_{s} B$-containing clone was apparent. (4) Transformation with either $n f_{S A}$ or $n f s B$ increases sensitivity of all strains to a similar level.

\subsubsection{EDTA treatment and nitrofuran susceptibility}

EDTA $(0.2 \mathrm{mM})$ results in a nonspecific increase in permeability in $E$. coli (Leive, 1965). To test if the elevated resistance of JVA6 and two of the "third-step" mutants, JVE2 $\alpha$ and JVE2 $\beta$, was due to decreased cellular permeability, cells were treated with EDTA and a susceptibility disk assay was performed in duplicate. Related strains were tested as well, including wild-type AB1157 and the parental strains JVA1, JVE1 and JVE2. Susceptibility to nitrofurazone and furazolidone was not affected in any of the strains by the EDTA treatment (data not shown).

\subsubsection{Induction of nitroreductase by paraquat and salicylate.}

Figure 2.4 illustrates the NADH and NADPH-dependent nitroreductase activity of AB1157, SIL41 and JVQ1 before and after paraquat and salicylate induction. JVQ2 is not included as it has very little nitroreductase activity $(<2 \mathrm{nmol} / \mathrm{min} / \mathrm{mg})$ in both cases.

The NADPH:nitroreductase activity (uninduced) of AB1157 and SIL41 was similar $(\sim 32 \mathrm{nmol} / \mathrm{min} / \mathrm{mg})$ and significantly greater than JVQ1 $(\sim 7 \mathrm{nmol} / \mathrm{min} / \mathrm{mg})$. AB1 $157 \mathrm{had}$ the highest level of NADH:nitroreductase activity $(11.6 \pm 2 \mathrm{nmol} / \mathrm{min} / \mathrm{mg})$, followed by JVQ1 $(5.9 \pm 3 \mathrm{nmol} / \mathrm{min} / \mathrm{mg})$. The NADH:nitroreductase activities of AB1 157 and 


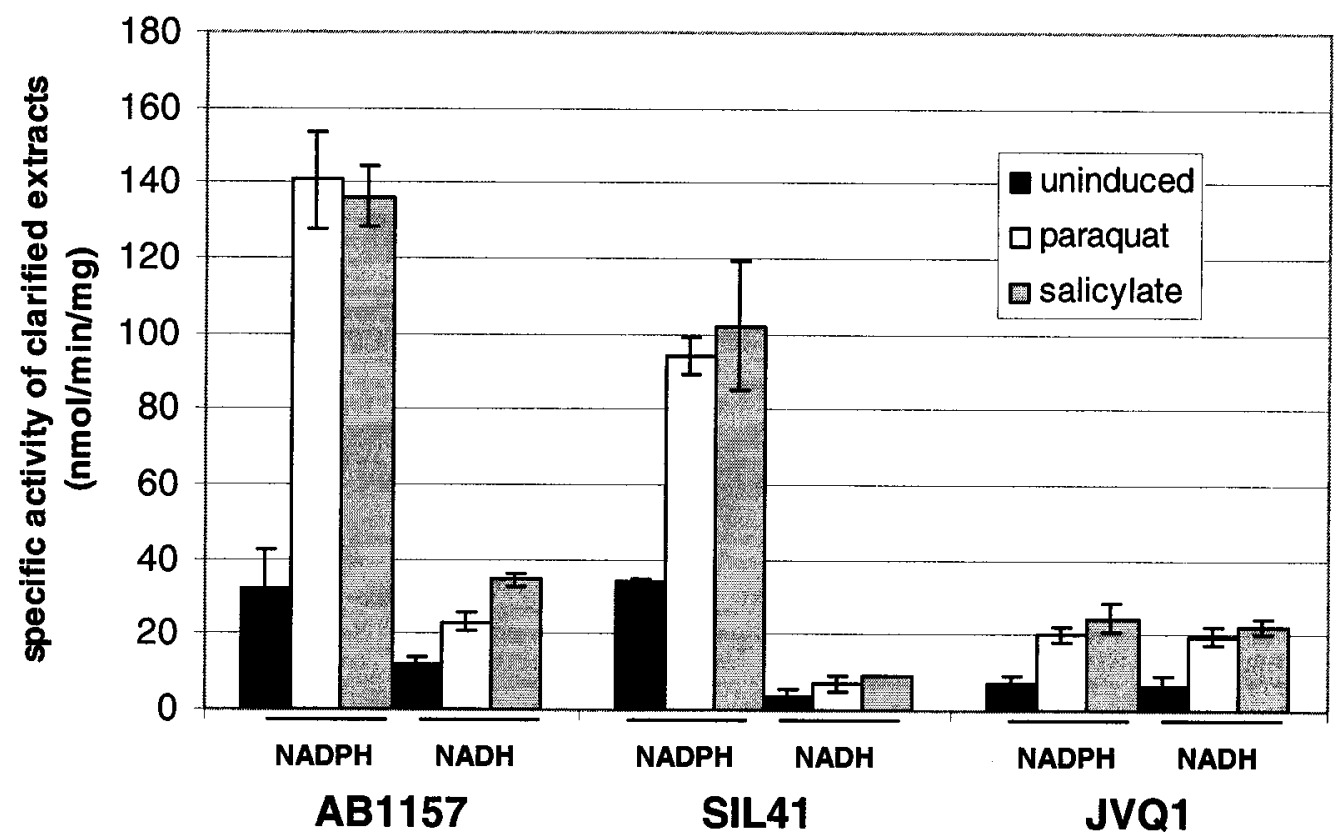

Figure 2.4 The inducibility of $\mathrm{NAD}(\mathrm{P}) \mathrm{H}$ associated nitroreductase activity with paraquat and salicylate treatment in cellular extracts of $E$. coli AB1157 and its derived mutants. SIL41 lacks functional NfsB, and JVQ1 lacks functional NfsA. JVQ2, lacking both NfsA and NfsB, had too little nitroreductase activity to assay and thus is not included here. Error bars represent the confidence interval of nitroreductase activity at a significance level of 0.05 as derived from the standard deviation of four trials. 
SIL41 were significantly less than those obtained with NADPH, about one-third and one-tenth respectively, while similar activities were observed in JVQ1 when either $\mathrm{NADH}$ or NADPH were used as cofactors.

Both paraquat and salicylate induction led to an increase in total oxygen-insensitive nitroreductase activity in AB1157, SIL41 and JVQ1. At the concentrations used, the inducers yielded similar levels of nitroreductase activity. For AB1157 and SIL41, induction of the NADPH associated activity was especially apparent relative to NADH:NR activity, while JVQ1 had nearly equal levels of NADH and NADPH associated nitroreductase activity.

A comparison of NADPH:nitroreductase activities demonstrates about a 4-fold induction in AB1157, and an approximate 3-fold induction in each SIL41 and JVQ1 following treatment with either paraquat or salicylate. While the uninduced nitroreductase activity of SIL41 and AB1 157 is similar, about $32 \mathrm{nmol} / \mathrm{min} / \mathrm{mg}$, the induced level of SIL41 $(\sim 100 \mathrm{nmol} / \mathrm{min} / \mathrm{mg})$ is approximately one-third less than AB1 $157(\sim 140 \mathrm{nmol} / \mathrm{min} / \mathrm{mg})$. The induced NADPH:nitroreductase activity of JVQ1 $(\sim 20 \mathrm{nmol} / \mathrm{min} / \mathrm{mg})$ is significantly less than for both SIL41 and AB1 157.

The induced NADH:nitroreductase activity in AB1157 ( 20-35 nmol/min/mg) is approximately $2-3$ fold greater than the uninduced activity $(11.6 \pm 2 \mathrm{nmol} / \mathrm{min} / \mathrm{mg})$. Induced NADH associated activity in JVQ1 is essentially the same as for NADPH activity, about $20 \mathrm{nmol} / \mathrm{min} / \mathrm{mg}$, which is similar to the NADH:nitroreductase levels of induced AB1 157. The NADH:nitroreductase levels in SIL41 are very low even after 
induction, $<10 \mathrm{nmol} / \mathrm{min} / \mathrm{mg}$, and although some induction may exist, the low activity level precludes making reliable comparisons. 


\subsection{Discussion}

\subsubsection{Isolation of nitrofuran resistant strains}

The stepwise manner in which furazolidone-resistant mutants were obtained in this study was comparable to that previously used to select mutants resistant to nitrofurazone (McCalla et al., 1970) and nitrofurantoin (Breeze and Obaseiki-Ebor, 1983; Sastry and Jayarman, 1984). Selection of E. coli K-12 nitrofuran-resistant strains on medium supplemented with furazolidone, nitrofurazone, and nitrofurantoin consistently resulted in two discrete steps of increased resistance. As compared to the parent strain $\mathrm{AB} 1157$ which is resistant to $5 \mu \mathrm{g} / \mathrm{ml}$ nitrofurazone, first and second step mutants selected on furazolidone were resistant to nitrofurazone concentrations of $15 \mu \mathrm{g} / \mathrm{ml}$ and $35 \mu \mathrm{g} / \mathrm{ml}$, respectively; similar levels of resistance were observed in the mutants NFR402 and NFR502 that were initially selected on nitrofurazone (McCalla et al., 1970; 1978). Biochemical analysis of the nitrofurazone resistant mutants NFR402 and NFR502 suggested that the stepwise resistance of $E$. coli to nitrofurans is caused by the sequential inactivation of the proteins NfsA and NfsB: NfsA activity is absent from both the first step mutant, NFR402, and the second step mutant, NFR502, while NfsB activity is present only in NFR402 (Bryant et al., 1981). DNA sequence analysis of a large number of nitrofuran resistant mutants in this study strengthens this observed pattern and strongly suggests that two step resistance is a consequence of an obligatory first step event, mutation in $n f s A$, followed by mutational inactivation in $n f_{s} B$. Further evidence that nitrofuran resistance occurs through an ordered inactivation of $n f s$ genes can be found in the interesting resistance characteristics of SIL41. SIL41 was constructed by crossing an 
Hfr $n f_{S} A n f_{S} B$ double mutant and $\mathrm{AB} 1157\left(n f s A^{+} n f_{s} B^{+}\right)$(Bryant et al., 1981). The resulting $n f_{s} A^{+} n f_{s} B$ strain, in which the $n f s B$ gene was inactivated by an amber mutation at $\mathrm{C}^{424}$, has a level of nitrofurazone resistance that is similar to the wild-type strain, suggesting that inactivation of $n f s B$ is phenotypically silent in the presence of a wild-type $n f s A$ gene. However, in contrast to wild-type bacteria, SIL41 can attain a high (second step) level of nitrofurazone resistance in a single step that presumably corresponds to inactivation of $n f s A$.

\subsubsection{Characterization of the nitrofuran resistant mutants}

The genetic characterization of these mutants corroborates previous biochemical studies suggesting that $\mathrm{NfsA}$ and $\mathrm{NfsB}$ nitroreductase activity is necessary for the majority of nitrofuran biological activity under aerobic conditions (Bryant et al., 1981; McCalla et al., 1978). Furthermore, by transforming strains with vector-encoded NfsA and $\mathrm{NfsB}$, these proteins are shown to be sufficient for maximal nitroreductase activity. Evidence that the sensitivity of a cell to nitrofurans is dependent on the functional status of NfsA and NfsB was shown by introducing cloned $n f s A$ and $n f s B$ into AB1157 (wildtype) and its nitroreductase deficient derivatives. Significant increases in the cleared zone around the nitrofurazone impregnated filters were observed in the strains AB1157, JVQ1 and JVQ2 upon introduction of pTAA $(n f s A)$ or pTAB $(n f s B)$. The increases in AB1 157 and JVQ1 upon the introduction of pTAB were presumably due to the multiple copies of the plasmid-encoded gene. The larger increase in sensitivity observed in the transformed first and second step mutants is attributable to a restoration of nitroreductase activity. While the percent increase in sensitivity was far greater in the mutants than in 
the wild-type, the absolute sensitivity of the transformants, as indicated by the diameter of the cleared zone in the lawn, was similar to AB1157 transformants. This maximum diameter could represent a limit either for the chemical's diffusion, or for cellular sensitivity to nitrofurazone.

Of the mutants selected in this study, three second step mutants did not have a mutation in $n f s B$ (Table 2.2) and one had a base substitution downstream of the coding region in the putative transcription terminator (Figure 2.1). Confirmation of the resistance of these mutants to elevated concentrations of nitrofurazone and furazolidone eliminates the possibility of mutant reversion. The complete sequence of $n f s B$ in these mutants, including the -35 region, was determined to be wild-type. Confirmation that this $n f s B$ fragment contained a functional promoter was achieved by demonstrating CAT activity in the plasmid pKK232-8, which contains a promoterless CAT gene, upon insertion of the putative promoter region of $n f s B$. NfsB is normally sufficient for biological activation of nitrofurans: sensitivity to nitrofurazone is increased upon the introduction of vector-encoded NfsB in both wild-type and mutant $E$. coli cells. Thus, the increased resistance demonstrated by these mutants suggests that additional factors may modulate nitrofuran resistance. Possible explanations include: (1) a nitroreductase enzyme distinct from NfsA or NfsB; (2) a factor mediating transport of the nitrofuran into the cell; or (3) a trans acting factor with which interaction is essential for full nitroreductase activity. In support of the first explanation, a third oxygen insensitive nitroreductase component, $\mathrm{IB}_{2}$, was described by Bryant and coworkers (Bryant et al., 1981). It is conceivable that a minority of second step mutants could be due to 
inactivation of this third minor component of nitroreductase. The last two possibilities may apply to JVA6; however, it is unlikely that transport of the nitrofuran into the cell is a factor, as treatment with $0.2 \mathrm{mM}$ EDTA does not yield greater sensitivity of JVA6 to furazolidone or nitrofurazone. EDTA at noninhibitory levels causes a non-specific increase in the permeability of $E$. coli (Leive, 1965), including to nitrofurans (ObaseikiEbor, 1984).

Beyond the initial two levels of resistance, mutants isolated on nitrofurantoin and furazolidone appear to have nitrofuran tolerance properties not shared by nitrofurazoneresistant mutants. Third step furazolidone-resistant mutants displayed inconsistent and non-uniform resistance when plated on nitrofurazone medium. The third level of resistance observed in our studies is similar to that described in the higher order nitrofurantoin-resistant mutants observed by Breeze and Obaseiki-Ebor (1983). Those workers showed that putative third and fourth step mutants selected on nitrofurantoin did not display cross-resistance to nitrofurazone; rather, they were characterized by a level of nitrofurazone resistance that was comparable to second step mutants. It is possible that the principal mode of toxic action of the reactive metabolites derived from nitroreduction differs between nitrofuran derivatives. For example, nitrofurans have a variety of toxic actions including genotoxicity, metabolic effects, effects on nucleotide pools, and formation of active oxygen species (McCalla et al., 1975b; Tu and McCalla, 1975) although the bacteriostatic effect of nitrofurans appears to correlate primarily with the extent of chromosomal damage (Jenkins and Bennett, 1976; Lu and McCalla, 1978). Alternatively, the participation of other, as yet uncharacterized, nitroreductase activities 
may contribute to the reduction of a subset of nitrofuran derivatives (e.g. furazolidone and nitrofuranoin, but not nitrofurazone) in the absence of NfsA and NfsB activity. A third possibility is that the high levels of unreduced nitrofurazone in higher order nitroreductase mutants may have an inhibitory effect on bacterial metabolism when mutants are plated on nitrofurazone medium (Tu and McCalla, 1975). Obaseiki-Ebor (1984) suggested that high-level nitrofurantoin resistant mutants were due to decreased permeability of the drug; however, mutants selected for nitrofurantoin resistance were not affected by EDTA treatment (Obaseiki-Ebor, 1984). Similarly, decreased permeability to furazolidone does not appear to be a factor for the higher level mutants tested in this study, as treatment with EDTA did not increase sensitivity of the mutants. Interestingly, Obaseiki-Ebor (1984) observed that EDTA treatment increased the nitrofuran susceptibility of aminoglycoside resistant mutants cross resistant to high levels of nitrofurantoin, suggesting another resistance mechanism for nitrofurans. A study by Morozov and colleagues (1994) also indicated that nitrofuran resistance is attributable to a different mechanism in these cross-resistant aminoglycoside mutants.

\subsubsection{IS element hotspots are likely present in the $n f s B$ gene}

Among the 49 mutations in the $n f s B$ gene of the furazolidone-resistant mutants isolated in this study, $26(53 \%)$ were derived from the insertion of one of the insertion elements IS1, IS2 or IS5 (Table 2.3). Transposition is usually a rare event, occurring at frequencies of $10^{-7}$ to $10^{-5}$ per generation, with only $5-15 \%$ of all spontaneous mutations being attributed to IS elements (Galas and Chandler, 1989). Although there is a high degree of variability in the copy number and presence of IS elements in $E$. coli strains 
(Boyd and Hartl, 1997), all IS element types have fixed locations on the chromosome of $E$. coli $\mathrm{K}-12$ strains, as well as having additional strain specific locations (Birkenbihl and Vielmetter, 1989; Boyd and Hartl, 1997). All of the IS elements characterized in this study have been mapped in various $E$. coli strains (Birkenbihl and Vielmetter, 1989; Boyd and Hartl, 1997). Distribution of IS elements in a host genome is nonrandom, and though, in part, this must be due to positional constraints imposed by consequences on the host's fitness, it is also a result of insertion preference at certain sequences. Clustering of particular IS families to certain chromosomal locations is believed to result from local hopping, a process in which local transposition events give rise to daughter IS elements inserted in proximity $(<10 \mathrm{~kb})$ to the parent element (Boyd and Hartl, 1997; Craig, 1997). The $n f s B$ gene is located at $13 \mathrm{~min}$. on the $E$. coli chromosome (Blattner, 1997). IS5, at $13.1 \mathrm{~min}$. , is the only element identified in this study to be located near $n f s B$ in the three mapped $E$. coli K-12 strains (Birkenbihl and Vielmetter, 1989). Proximity of IS5 to $n f s B$ on the chromosome, in addition to the probable IS 5 hotspot in $n f s B$ (discussed below), and the high transposition rate observed for IS5 (Naas et al., 1994) could account for the large number of $n f_{S B} B$ mutants (19\%) containing IS5 in this study. A hotspot for IS 5 after $\mathrm{G}^{43}$ is evident in $n f_{S} B$ : ten independent mutants that contained IS 5 at the same site were isolated in this study. The target sequence for IS 5 in $n f s B, \mathrm{AAGG}^{43}$, does not conform to the previously described IS 5 target site consensus sequence $\mathrm{C}(\mathrm{A} / \mathrm{T}) \mathrm{A}(\mathrm{G} / \mathrm{A})$ (Engler and van Bree, 1981). However, of the 12 sequences within the coding region of $n f s B$ that did match this consensus sequence, two, $\mathrm{CTAA}^{41}$ and $\mathrm{TTTG}^{49}$, flanked the insertion point of IS5. 
All IS elements demonstrated specificity for insertion in the regions proximate to either terminus of $n f s B$, suggesting that despite the variable target specificity of IS elements there may be some common determinants in target site selection. In $n f_{s} B$, the IS element most frequently observed was IS1. IS 1 is often associated with regional preferences for segments of DNA, of approximately $100 \mathrm{bp}$, into which the IS elements randomly insert themselves (Galas et al., 1987; Meyer et al., 1980; Zerbib et al., 1985). Several studies have shown that IS 1 has a preference for insertion into stretches of A+T rich sequences (Matsutani, 1997; Meyer et al., 1980; Zerbib et al., 1985). It has been suggested that IS 1 , like RNA polymerase, may require regions high in $\mathrm{A}+\mathrm{T}$ for their low helix stability (Matsutani, 1997; Meyer et al., 1980); therefore, $\mathrm{A}+\mathrm{T}$ rich promoter regions could be expected to be targets for IS1. Among the mutants described in the current study, one mutant had IS 2 integrated upstream of the coding region of $n f_{s} B$. The insertion of IS2 upstream from a gene can terminate transcription by the Rho-sensitive termination sites and transcription terminators present in these elements (Das et al., 1977).

Eight mutants with IS 1 elements in $n f s B$ had IS 1 inserted within the first 165 bases of the coding region, possibly due to the proximity of the $\mathrm{A}+\mathrm{T}$ rich promoter region (Matsutani, 1997). IHF induced DNA bending has also been suggested to be a signal for ISI insertion (Galas and Chandler, 1989; Galas et al., 1987; Prentki et al., 1987). Although there is no obvious IHF binding site near this region, there is a high density of homopolymeric runs of adenine and thymine which, according to the wedge model of 
DNA conformation, could promote bending of DNA (Goodrich et al., 1990; Hales et al., 1994; Koo et al., 1986; Ulanovsky and Trifonov, 1987).

Target site sequence specificity may sometimes be attributed to protein consensus sequences. In particular, $E$. coli integration host factor (IHF), a protein that induces DNA bending, has been implicated in some instances of IS1 site recognition (Galas et al., 1987; Prentki et al., 1987). The $n f_{s} B$ gene contains one site at bases $640-652$ that conforms to an IHF binding site consensus sequence WWWCAWNANNTTA (W is A or T) (Goodrich et al., 1990; Hagerman, 1990). Notably, all IS elements, five IS1 and one IS2, that integrated near the 3' end of $n f s B$ were inserted within $100 \mathrm{bp}$ of this consensus sequence, and two integration sites were exactly nine bases on either side.

\subsubsection{Implications of the mutations for the NfsB enzyme}

The mutational distribution of the furazolidone and nitrofurazone resistant mutants in the $n f s B$ gene (refer to Figure 2.1) implicates particular residues as having functional significance in the $\mathrm{NfsB}$ protein. $\mathrm{NfsB}$ shares $88 \%$ amino acid sequence identity with homologous nitroreductases isolated from $S$. typhimurium (Watanabe et al., 1989 ) and $E$. cloacae (Bryant et al., 1991), and 33\% identity with the homologue FRaseI, the major flavin oxidoreductase of Vibrio fischeri (Zenno et al., 1994), compared with $16 \%$ identity with NfsA. The sequences of RdxA and FrxA from Helicobacter pylori (Goodwin et al., 1998), Nox from T. thermophilus (Hecht et al., 1995), three enzymes from Bacillus subtilis (Kunst et al., 1997), and DrgA from the cyanobacterium Synechocystis sp. PCC 6803 (Elanskaya et al., 1998) also share some amino acid sequence identity $(>20 \%)$ with $\mathrm{NfsB}$. These proteins have all been shown to have 
nitroreductase activity, and inactivation of $\operatorname{RdxA}$, FrxA and DrgA has been associated with resistance to nitroheterocyclic drugs. Moreover, the sequential inactivation of the $H$. pylori nitroreductase genes $r d x A$ (HP0954) and frxA (HP0642) has recently been associated with the development of metronidazole resistance (Jeong et al., 2000). Although the overall similarity of these sequences to NfsB is only about $20-30 \%$, the identity between proteins in the 14 residue stretch corresponding to Ser-37 to Val-50 of NfsB (Figure 2.5) is high: it is identical for E. coli, S. typhimurium and E. cloacae; and $V$. fischeri shares $78 \%$ identity with NfsB. Most of the other putative NfsB-like homologues share protein identities over $50 \%$ within this region (NfsA is $28 \%$ similar), with the exceptions of FrxA (36\%) and DrgA (43\%) (Table 2.5). It is interesting that of the 17 $n f s B$ mutants with missense mutations, $7(41 \%)$ are located in this region. This region serves as a fingerprint for the identification of NfsB-like proteins, especially the invariant Trp-46. The other missense mutations mainly affected conserved residues: two such residues (Asn-71 and Arg-207) were conserved in $8(73 \%)$ and $9(82 \%)$, respectively, of the 11 probable homologues shown in Table 2.5, while the other amino acids altered by mutation in $\mathrm{NfsB}$ were conserved in at least four of the proteins. E. coli $\mathrm{NfsB}$, the NfsBlike nitroreductases from $S$. typhimurium and $E$. cloacae, and FRaseI from $V$. fischeri constitute a protein family (Zenno et al., 1996) that can be extended to include the proteins from H. pylori, B. subtilis, T. thermophilus and Synechocystis sp that have nitroreductase activity and share identity with $\mathrm{NfsB}$, especially within the 14 residue fingerprint. With the exception of RdxA, which exclusively uses NADPH, all of these enzymes, like NfsB, can use either NADH or NADPH as a source of reducing 
เ

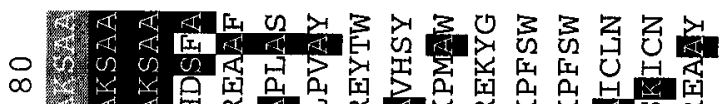

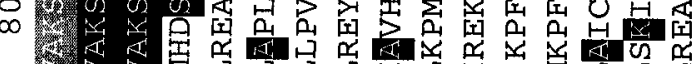

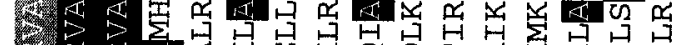

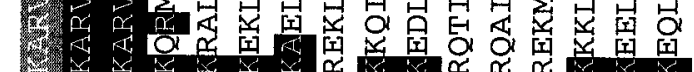

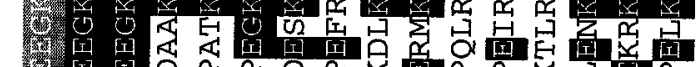

त

6.

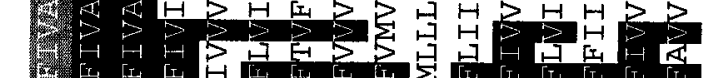

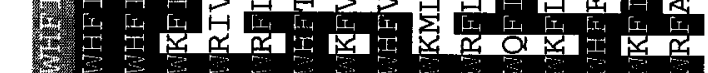

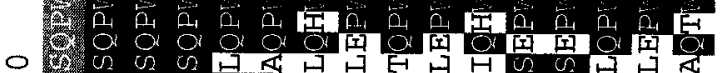

$\circ$

아

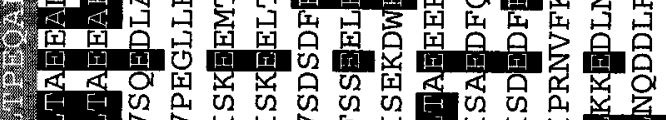

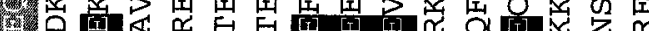

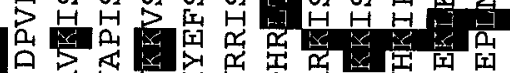

9020201

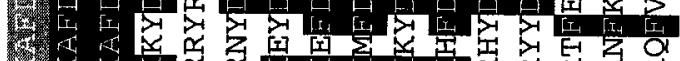

* W

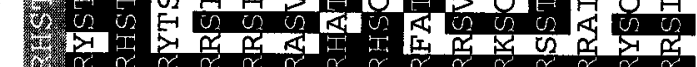

$\Rightarrow$ वे

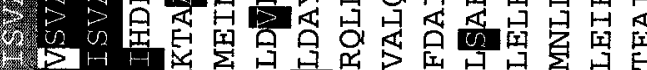

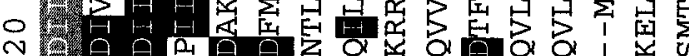

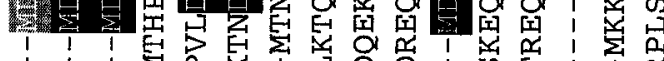

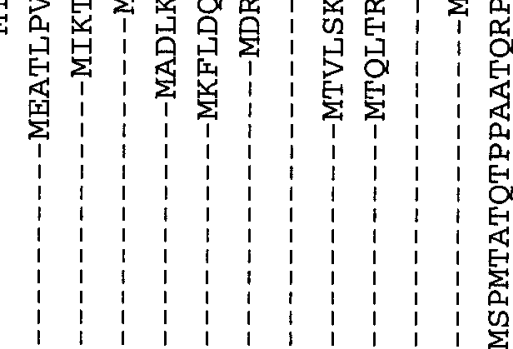

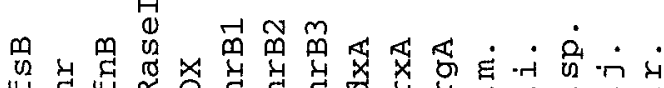

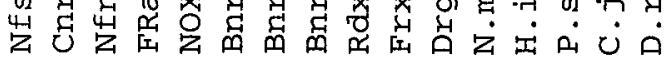

A

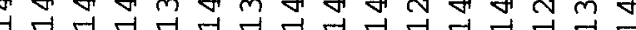

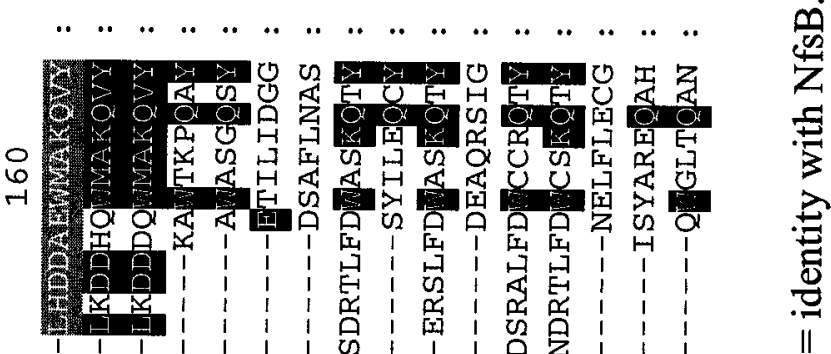

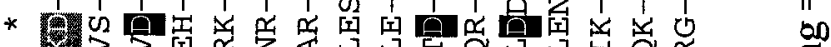

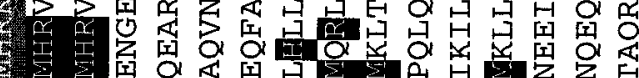

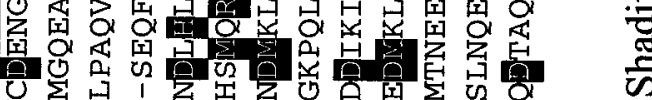

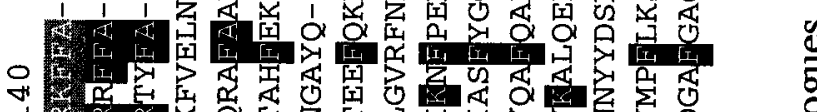

अ

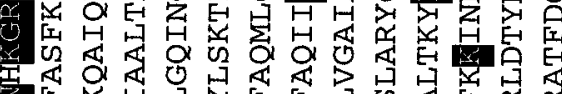

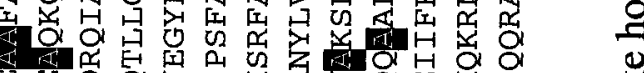

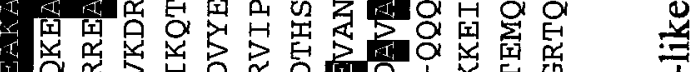

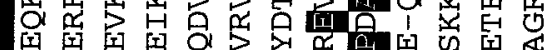

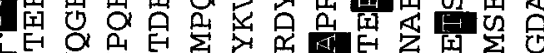

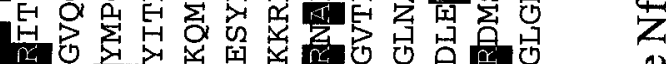

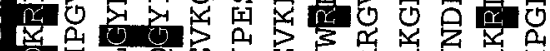

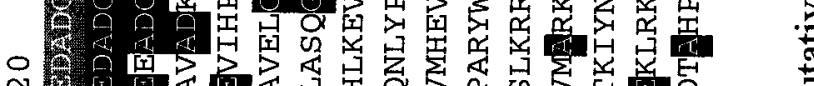

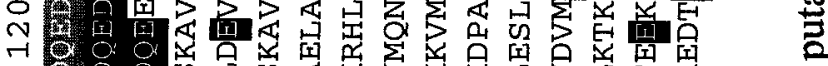

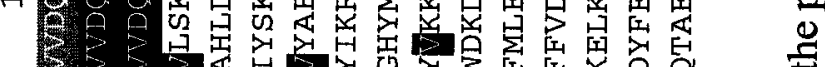

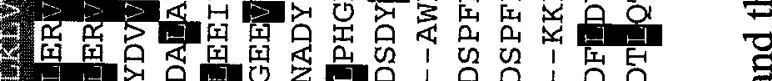

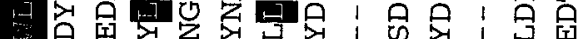

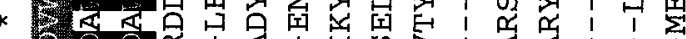

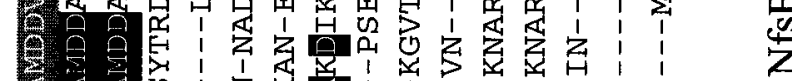

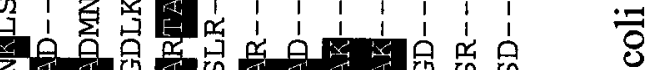

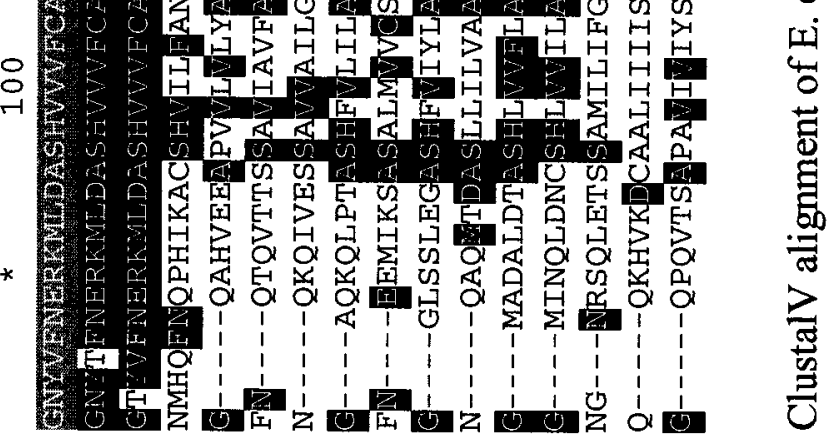

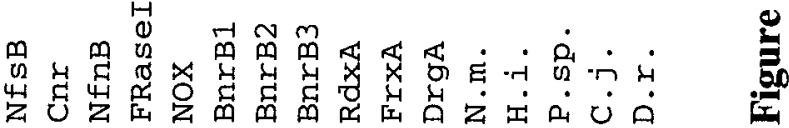




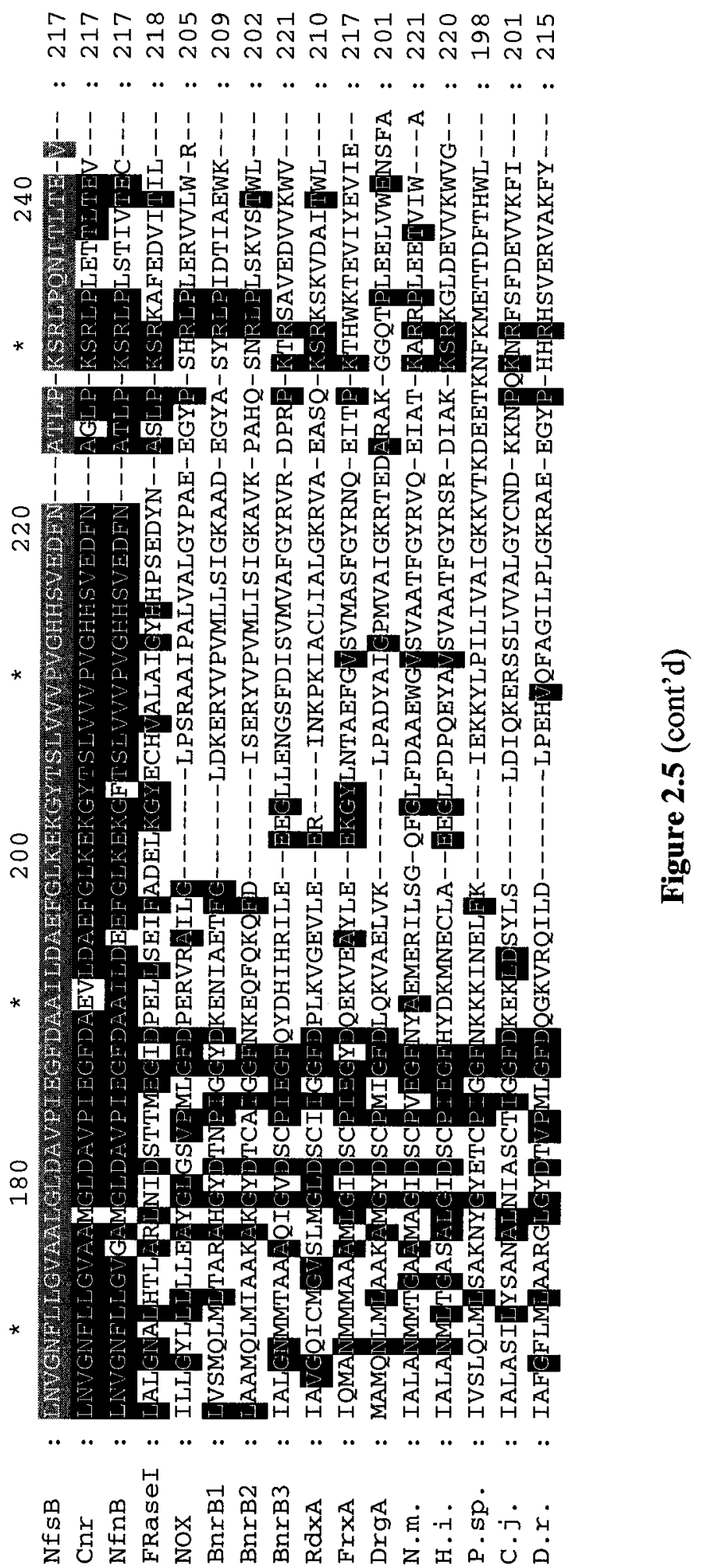


Table 2.5 Sequence identities of putative NfsB homologues and paralogues. Percent identity of the NR fingerprint region (Ser-37 to Val-50) is included.

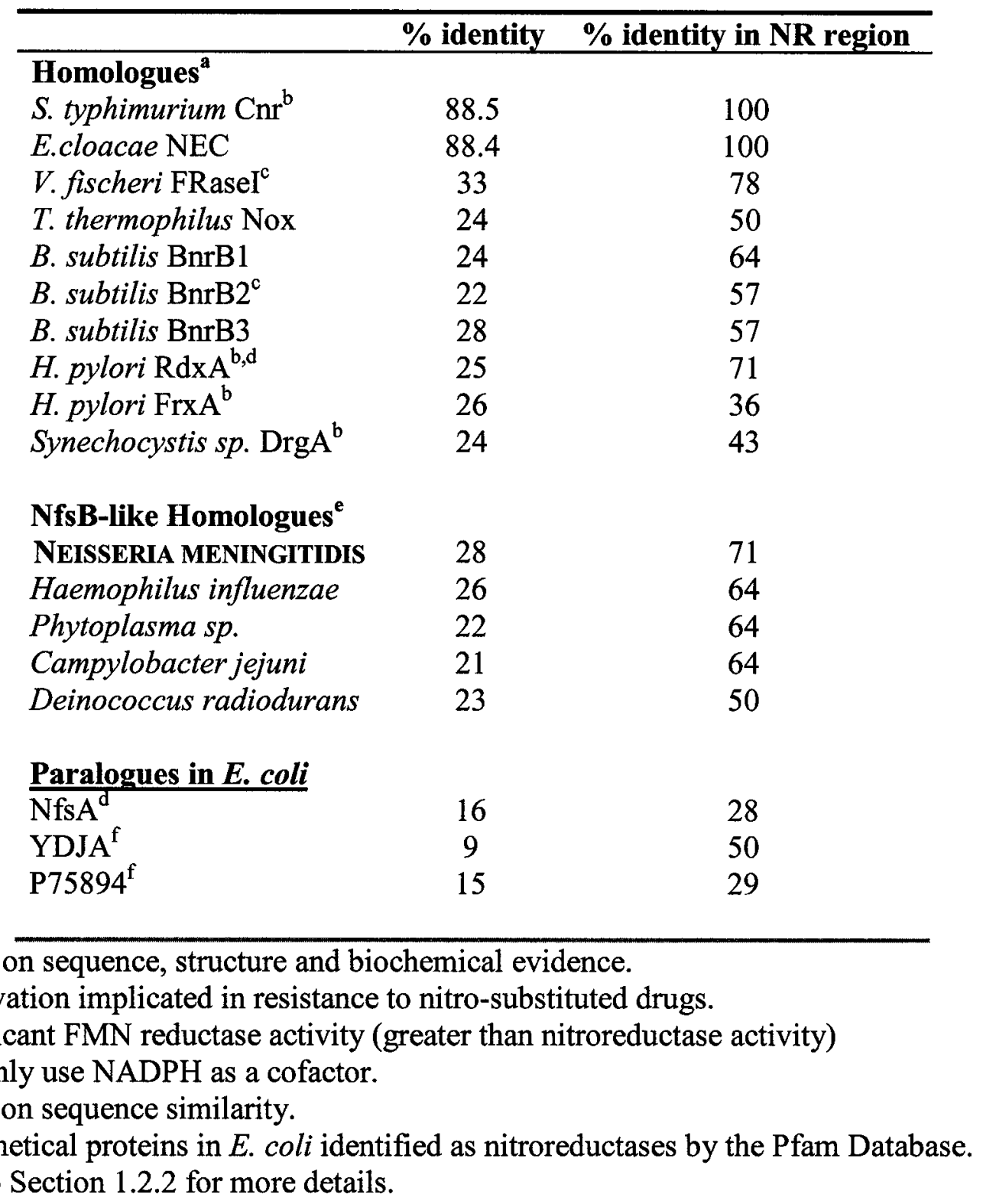


equivalents, usually with a slight preference for $\mathrm{NADH}$. It is more likely that RdxA is an orthologue of NfsB than NfsA as there is only $15 \%$ amino acid identity with NfsA (vs. $25 \%$ with $\mathrm{NfsB}$ ), and its sequence is more characteristic of a NfsB homologue, particularly within the fingerprint region. As genome projects are completed, numerous hypothetical proteins that appear to fit a nitroreductase fingerprint arise. Five hypothetical proteins that are likely to be NfsB homologues based on sequence similarity are shown in Table 2.5, but many more exist (for details refer to Section 1.2.2).

\subsubsection{NfsB: a putative member of the SoxRS/MarRAB stress response}

With numerous genome projects underway, the newly defined NR family of proteins promises to extend indefinitely. However, the natural function and substrate(s) for nitroreductases remain unknown. The GenProtEC E. coli genome and proteome database (http:// genprotec.mbl.edu/start) states that the physiological roles for NfsA and $\mathrm{NfsB}$ are anaerobic respiration and drug/analogue sensitivity, respectively. The accuracy of this information is questionable, however, as the cited literature (McCalla et al., 1978; Morozov et al., 1994) does not identify the physiological roles of these proteins. Both NfsA and NfsB certainly cause drug/analogue sensitivity, but a suicidal role is unlikely to be their primary function. Furthermore, the apparent redundancy of nitroreductase enzymes in $E$. coli (two putative oxygen-sensitive and three oxygen-insensitive enzymes) suggests a variety of in vivo roles. The inability to obtain mutants without oxygensensitive nitroreductase activity or even without the third putative oxygen-insensitive enzyme (Bryant et al., 1981; McCalla et al., 1975) suggests that these components are essential proteins. On the other hand, inactivation of the characterized nitroreductases in 
E. coli (NfsA and NfsB) and S. typhimurium (SnrA and $\mathrm{Cnr}$ ) has no apparent negative effect on cellular viability or function under laboratory conditions, and easily cultured clinical isolates of $H$. pylori metronidazole resistant mutants contain inactivated $\mathrm{RdxA}$ and FrxA enzymes.

It is interesting that there often appears to be more than one protein from the NR family within an organism. This leads to speculation on the evolutionary origin of the NR family. It could be that there was duplication of the hypothetical ancestral NR gene, after which each gene was adopted for a specific process, resulting in a paralogous relationship. This organism would thus represent the common ancestor for organisms containing the NRs. However, horizontal transfer could also be a contributing factor and a reasonable explanation for what is apparently an evolutionarily widespread distribution of NRs. A strong candidate for horizontal transfer is transposable element (TE) activity, a possibility that resonates with the large number of IS elements observed in the $n f_{s} A$ and $n f s B$ genes of the nitrofuran resistant mutants described in this study.

It is possible that nitroreductases have physiological functions and substrates that are yet to be determined. For instance, NfsA uses NADPH exclusively as a cofactor, a characteristic often associated with biosynthetic processes, while NfsB is unusual in that it can use either NADH or NADPH. NADH is usually a cofactor in catabolic processes, so the ability to use both cofactors is suggestive of a broader physiological role for NfsB. The identification of $\mathrm{NfsB}$ and its homologues has largely been through studies of drug resistance (E. coli, H. pylori, Synechocytis sp.) or activation (S. typhimurium, E. cloacae). It is interesting that several other bacterial species with putative NfsB 
homologues are also of clinical importance (ex. N. meningitis, H. influenzae, C. jejuni) or are associated with extreme environments (ex. T. thermophilus, D. radiodurans). The nitroreductase activity that bioactivates many clinically important synthetic antimicrobial agents and manmade (primarily) environmental contaminants may very well provide a protective response for the organism against other compounds that do not result in the cell's death. This catabolic role is consistent with NADH associated activity. That NfsB may play a protective role physiologically is consistent with its involvement in responses to environmental stresses, such as oxidative stress (see below) or starvation (A. Matin, personal communication).

Recently, $n f s A$ and $n f s B$ were shown to be putative members of the multipleantibiotic-resistance (marRAB) regulon (Barbosa and Levy, 2000). The $n f s A$ (Liochev et al., 1999; Paterson et al., 2002) and $n f_{s} B$ (this study) genes are positively regulated as part of the closely associated soxRS regulon. The soxRS and marRAB regulons respond to and provide protection against cellular oxidative stress and numerous antimicrobial agents, respectively. The activators of these regulons, SoxS and MarA, are homologous proteins (Martin et al., 1999). Indeed, the $\operatorname{sox} R S$ and marR $A B$ regulons are often regarded as a single regulon. The binding sites for these promoters are generally referred to as the marbox, though the determinants of this element are not well understood (Martin et al., 1999). Indeed, genes activated by SoxS are commonly responsive to MarA and vice versa (Martin et al., 1999). There are some exceptions to this overlap, however, and MarA and SoxS are independently regulated, with neither protein affecting the expression of the other (Barbosa and Levy, 2000). As well, the individual regulons can be 
selectively induced with certain chemicals, for instance soxRS by paraquat and marR $A B$ by salicylate (Martin et al., 1999).

NfsA is a regulated member of the soxRS regulon (Liochev et al., 1999; Paterson et al., 2002). Nitroreductase activity associated with NfsA was shown to increase significantly when treated with paraquat, but while Liochev and coworkers suggested that NfsB might be slightly induced by paraquat, it wasn't within the scope of their study to resolve this possibility. Recently, increased expression of $n f_{s} A$ and $n f s B$ was shown to result from the constitutive expression of MarA, and thus both are considered members of the marRAB regulon (Barbosa and Levy, 2000). In the current study, E. coli strains that varied with respect to their $n f s A$ and $n f s B$ genotypes were treated with paraquat and assayed for induced nitroreductase activity in order to discern whether $n f s B$ is a member of the soxRS regulon. AB1157, the wild-type strain, obviously has oxygen insensitive nitroreductase activity attributable to both $\mathrm{NfsA}$ and $\mathrm{NfsB}$ (and to a much lesser degree the third nitroreductase component $\left.\mathrm{IB}_{2}\right) ; \operatorname{SIL} 41(\Delta n f S B)$ has an intact $n f s A$ gene, but an inactive $n f s B$ gene; JVQ1 ( $\Delta n f s A)$ has an intact $n f s B$ gene, but an inactive $n f s A$ gene; and JVQ2 $(\Delta n f s A n f s B)$ is deficient in both genes and has negligible nitroreductase activity. Treatment with salicylate was included to confirm activation by MarA. The level of nitroreductase activity in JVQ2 was negligible and not noticeably induced with paraquat or salicyclate, and thus only the three strains with measurable nitroreductase activity are discussed here. Paraquat induced nitroreductase activity in all three strains, although about one-fold less in SIL41 than in AB1157 (Figure 2.4). This suggests that $n f_{s} B$ is a regulated member of the soxRS regulon. Similar induction with salicylate confirmed $n f s B$ 
as a member of the marRAB regulon. As a member of the soxRS regulon, induction of $n f_{s} B$ would be expected to be present only in AB1157 and JVQ1, and induced nitroreductase activity in SIL41 would be solely attributable to $n f s A$. If $n f s B$ was not SoxS responsive then induction would not have been evident for JVQ1, and AB1 157 and SIL41 would have had similar levels of induced nitroreductase activity.

To further establish that increased activity upon induction with paraquat or salicylate was due to $n f s B$ and not $n f s A$, the different cofactor dependencies of NfsA and NfsB were exploited. That is, while NfsA is NADPH dependent, NfsB can use either NADH or NADPH. Therefore NADPH:nitroreductase activity reflects total activity, while NADH:nitroreductase activity is virtually attributable to $\mathrm{NfsB}$ alone. This difference in cofactor dependency is reflected in the nitroreductase activities of the uninduced extracts: AB1157 has approximately two-thirds less NADH than NADPH associated nitroreductase activity; SIL41 has trivial NADH:nitroreductase activity despite NADPH activity comparable to AB1157; and JVQ1 has similar levels of NADH and NADPH associated nitroreductase activities (Figure 2.4). That the $n f s B$ gene is a member of the soxRS/marRAB regulons is strongly suggested by the similar levels of NADH:nitroreductase activity that are observed for JVQ1 and AB1157, the lack of induction of NADH activity evident for SIL41, and the similar levels of NADH and NADPH nitroreductase activity that are apparent for JVQ1. The possibility that the third minor nitroreductase component, $\mathrm{IB}_{2}$, is responsible for the induced $\mathrm{NADH}$ :nitroreductase activity is rejected because JVQ2, which contains $\mathrm{IB}_{2}$ but not $\mathrm{NfsA}$ or $\mathrm{NfsB}$, has negligible nitroreductase activity both before and after induction. 
Although $n f_{s} B$ appears to be a member of the $\operatorname{mar} R A B$ and soxRS regulons, no obvious binding site, or marbox, for MarA or SoxS is apparent in the promoter region of the $n f s B$ gene based on the consensus sequence (AYnGCACnnWnnRYYAAAYn) (Martin et al., 1999). A gel shift assay and/or DNase footprinting could help to identify the putative MarA/SoxR binding site(s). 


\section{ChaPTER ThreE: THE KINETIC CHARACTERIZATION OF E. COLI NFSB}

\section{Site-directed mutagenesis and determinants of cofactor specificity.}

\subsection{Introduction}

The three dimensional structures of $E$. coli NfsB (Parkinson et al., 2000) and NfsB complexed with nicotinic acid (Lovering et al., 2001) were recently solved by Xray crystallography. Nicotinic acid, a nicotinamide analogue, represents only a component of the NAD(P)H cofactor; therefore, not all of the residues involved in cofactor binding have been determined. Structural determination of the NAD(P)H binding site in NEC, the Enterobacter cloacae homologue of NfsB, could not be obtained by NMR (Koder and Miller, 1998a), and unstable crystals of NADH complexed with FRaseI, the $V$. fischeri homologue of NfsB, prevented resolution of the cofactor binding site in this enzyme as well (Koike et al., 1998). Identification of residues in NfsB that are involved in the binding of cofactors could facilitate the engineering of a more specific enzyme, which would be an advantage for such applications as DEPT (see Section 1.3.1.2), or drug development (discussed in more detail in Chapter 4).

$\mathrm{NfsB}$ is unusual in that it does not display a strong preference for $\mathrm{NADH}$ or NADPH. NAD ${ }^{+}$and NADP ${ }^{+}$differ only by the presence of a phosphate group esterified to the 2'-hydroxyl group of ribose, and yet most enzymes have a strong preference for one of the coenzymes. Almost exclusively, $\mathrm{NAD}^{+}$is associated with degradations, and $\mathrm{NADP}^{+}$with reductive biosynthesis (Baker et al., 1992; Carugo and Argos, 1997a; Kaufman, 1993); however, the mechanism(s) through which enzymes discriminate between the two coenzymes remains poorly understood. Identification of NAD(P) ${ }^{+}$ binding domains is complicated by the lack of informative consensus sequences. The 
most frequently occurring structural motif is the Rossman fold, which is comprised of two parallel $\beta$-strands linked by an $\alpha$-helix (Carugo and Argos, 1997a); the most commonly cited consensus sequence for $\mathrm{NAD}(\mathrm{P})^{+}$binding is the GXGXXG/A motif (Wierenga et al., 1985), which overlaps the loop region between the $\beta$-strand and $\alpha$-helix. Still, it is often only a three dimensional structure of cofactor bound enzyme that can absolutely reveal the binding site of $\mathrm{NAD}(\mathrm{P})^{+}$(Carugo and Argos, 1997a,b; Kinoshita et al., 1999).

In this study, an expression system was developed to purify $E$. coli-NfsB. The seven residue region from Gly-153 to Leu-159 of the putative active site was modified by PCR mutagenesis through the introduction of 16 independent missense mutations. Wildtype NfsB and selected mutants were kinetically characterized. Most of these mutations resulted in a dramatic reduction of nitroreductase activity. The exception was the G158S mutant: this single Gly $\rightarrow$ Ser mutation decreased the enzyme's catalytic specificity for NADPH but not NADH. Similar results were obtained for a mutant that contained two mutations, one of which, Val-154 $\rightarrow$ Ala, is within this target region. These results suggest that the Gly-158 residue, and possibly other residues in this region, is important for the utilization of NADPH. A serendipitous $\mathrm{Phe} \rightarrow$ Ser mutation at residue 70 is also interesting. F70S did not influence cofactor use; but this mutant did display an increased turnover number, $\mathrm{k}_{\mathrm{cat}}$, for nitrofurazone, which may be indicative of a decrease in transition state energy. Here, the kinetic data obtained for the mutant enzymes are interpreted with consideration for possible perturbations in their secondary and tertiary structures, as revealed by fluorescence spectrophotometry and computer simulation, 
respectively. The structural and functional implications of these mutations are more thoroughly explored in Chapter 4, particularly with regard to the active site of NfsB. 


\section{$\underline{\text { 3.2 Methods and Materials }}$}

\subsubsection{Bacterial strains, plasmids and growth conditions.}

E. coli K-12 strain DH5 $\alpha$ [SupE44 lacU169 ( $\$ 80$ lacZAM15) hsdRI7 recAl

endA1 gyrA96 thi-1 relA1] (Jessee, 1986) is a standard laboratory strain used for most

plasmid manipulations. E. coli $\mathrm{K}-12$ strain $\mathrm{AB} 1157$ [F- thr-1 leu-6 thi-1 supE44 lacY1

galK2 ara-14 xyl-5 mtl-1 proA2 his-4 argE3 str-31 tsx-33 $\lambda^{-}$sup-37] (Bachmann, 1972)

was provided by Dr. D. Bryant, McMaster University, Hamilton, Canada. E. coli B strain GJ1158 (Bhandari and Gowrishankar, 1997) is a derivative of BL21 (FompTr ${ }_{B}^{-}{ }_{B}^{-} h s d S$ gal) (Studier and Moffat, 1986) with a proUp-T7 RNA polymerase fusion integrated at the malTPQ locus, allowing $\mathrm{NaCl}$-induced overexpression of cloned gene products.

The vectors used in this study were $\mathrm{pCR}^{\circledR} 2.1$ from the Original TA Cloning ${ }^{\circledR} \mathrm{Kit}$ (Invitrogen), pCYB3 from the IMPACT ${ }^{\mathrm{TM}}$ I Kit (New England Biolabs), pET23d (Novagen), pNEB193 (New England Biolabs), and pUC128 (Keen et al., 1988). The primers in this study are listed in Table 3.1.

Bacterial strains $\mathrm{DH} 5 \alpha$ and $\mathrm{AB} 1157$ were grown at $37^{\circ} \mathrm{C}$ in Luria-Bertani (LB) medium or on LBA (LB medium with $1.5 \%$ agar), obtained as a premixed powder from Canadian Life Technologies Inc. (Burlington, Canada). The NaCl-inducible strain GJ1158 was grown in LB broth with $\mathrm{NaCl}$ omitted (LB-N: $10 \mathrm{~g}$ tryptone, $5 \mathrm{~g}$ yeast extract per litre). Unless otherwise stated, GJ1158 was grown at $37^{\circ} \mathrm{C}$. When appropriate, medium was supplemented with $100 \mu \mathrm{g}$ ampicillin per $\mathrm{ml}$ and $40 \mu \mathrm{g}$ of 5-bromo-4chloro-3-indolyl- $\beta$-D-galactoside (Xgal) per ml. All chemicals and reagents were 


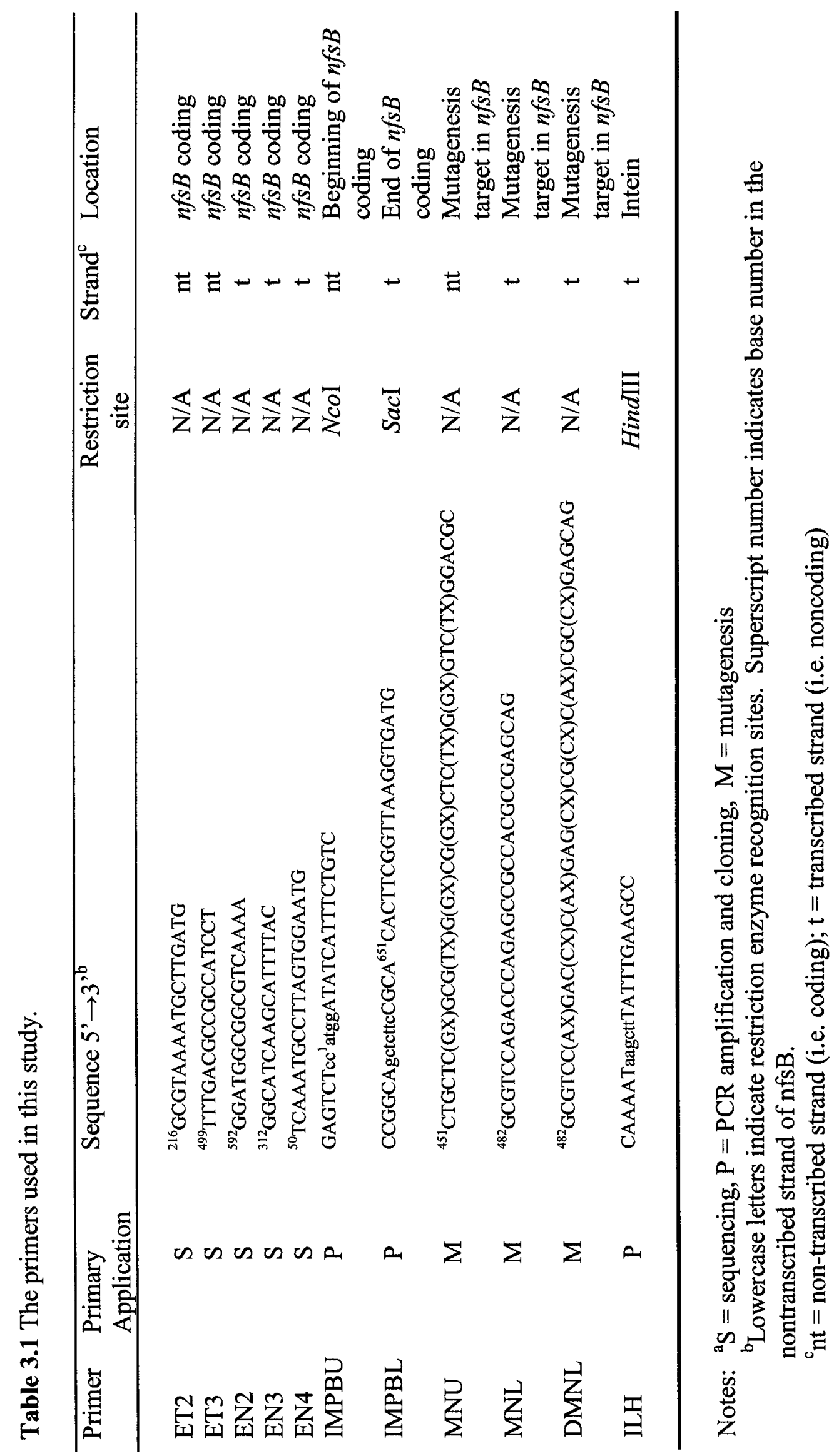


molecular biology grade or equivalent. Restriction endonucleases and modification enzymes were purchased from New England Biolabs or Gibco BRL.

\subsubsection{Manipulation of DNA.}

AB1 157 genomic DNA was isolated from a $5 \mathrm{ml}$ overnight culture. The cells were harvested by centrifugation and the pellet was resuspended in $1.9 \mathrm{ml} \mathrm{TE}(0.1 \mathrm{mM}$ Tris, $0.01 \mathrm{mM}$ EDTA, $\mathrm{pH} 8.0$ ). The cells were lysed with $0.5 \%$ SDS and $30 \mu \mathrm{g} / \mathrm{ml}$ proteinase $\mathrm{K}$ during a one hour incubation at $37^{\circ} \mathrm{C} . \mathrm{NaCl}(0.7 \mathrm{M})$ was added and mixed vigourously prior to the addition of $1 \%$ hexadecyltrimethyl ammonium bromide (CTAB) $/ 0.07 \mathrm{M} \mathrm{NaCl}$ (from a $10 \% \mathrm{CTAB}, 0.7 \mathrm{M} \mathrm{NaCl}$ stock solution) and the mixture was incubated for $10 \mathrm{~min}$ at $65^{\circ} \mathrm{C}$. The proteins and cellular debris were extracted with 5 $\mathrm{ml}$ of chloroform/isoamyl alcohol (24:1). RNase A (20 $\mu$ l of $10 \mathrm{mg} / \mathrm{ml})$ was added to the aqueous phase and incubated for $30 \mathrm{~min}$ at $37^{\circ} \mathrm{C}$. The remaining proteins and RNase $\mathrm{A}$ were extracted with $5 \mathrm{ml}$ phenol/chloroform/isoamyl alcohol (25:24:1). The DNA was precipitated from the aqueous fraction with 0.6 volumes of isopropanol, washed in $1 \mathrm{ml}$ of $70 \%$ ethanol, dried and resuspended in $50 \mu \mathrm{l}$ of TE.

Plasmid DNA was isolated either by using a Wizard ${ }^{\mathrm{TM}}$ miniprep kit (Promega), or by the boiling miniprep method (Holmes and Quigley, 1981) described in Section 2.2.7. If further purification was necessary it was done with a GeneClean ${ }^{\mathrm{TM}}$ kit (Bio 101 Inc.). Standard procedures were used for molecular cloning. Amplification product and restriction endonuclease analyses were done by agarose gel electrophoresis $(1 \%)$ in the presence of ethidium bromide. Transformation of $\mathrm{DH} 5 \alpha$ cells by ligation mixtures was accomplished by electroporation. The ligation mixes were purified with a GeneClean ${ }^{\mathrm{TM}}$ 
kit, and half was used to transform $40 \mu$ of host cells. Competent cells were prepared for electroporation by four successive washes of sterile distilled water and stored at $-80^{\circ} \mathrm{C}$ in $10 \%$ glycerol. Transformation of GJ1158 by constructs was achieved by calcium chloride transformation. Competent cells were prepared by a wash with one-quarter volume of $100 \mathrm{mM} \mathrm{MgCl}_{2}$, brought up in $1 / 20$ volume of $100 \mathrm{mM} \mathrm{CaCl}_{2}$, and stored at $80^{\circ} \mathrm{C}$ with $10 \%$ glycerol. Transformation was achieved by mixing $1-5 \mu$ of plasmid (about $0.5-2 \mu \mathrm{g}$ ) with $100 \mu \mathrm{l}$ of competent cells, incubation on ice for one hour, followed by heat-shock at $37^{\circ} \mathrm{C}$ for $5 \mathrm{~min}$, and recovery with gentle shaking at $37^{\circ} \mathrm{C}$ for up to one hour after the addition of $1 \mathrm{ml}$ LB-N broth.

\subsubsection{Amplification of the $n f s B$ gene.}

Amplification of a $656 \mathrm{bp}$ fragment containing the coding region of the $n f_{s} B$ gene was accomplished by PCR with the Expand ${ }^{T M}$ High Fidelity PCR System (Boehringer Mannheim Canada). The upper (IMPBU) and lower (IMPBL) primers (Table 3.1) contained 5' $\mathrm{NcoI}$ and SapI restriction sites, respectively, to facilitate cloning. The reaction mixture $(100 \mu 1)$ included the Expand High Fidelity buffer with $15 \mathrm{mM} \mathrm{MgCl}_{2}$, $0.2 \mathrm{mM}$ each deoxynucleoside triphosphate, $300 \mathrm{nM}$ of each primer, $1.7 \mathrm{U}$ of Expand High Fidelity enzyme mix, and approximately $0.5 \mu \mathrm{g}$ AB1 157 genomic DNA. The amplification consisted of a $3 \mathrm{~min}$ preincubation at $94^{\circ} \mathrm{C}$, a 7 min postincubation at $72^{\circ} \mathrm{C}$, and 30 repetitions of the following cycle: $15 \mathrm{~s}$ at $94^{\circ} \mathrm{C}, 30 \mathrm{~s}$ at $55^{\circ} \mathrm{C}, 1 \mathrm{~min}$ at $72^{\circ} \mathrm{C}$. The PCR product was purified using a High Pure PCR Product Purification Kit (Boehringer Mannheim Canada). 


\subsubsection{Cloning of $n f s B$.}

The $\mathrm{P}_{\text {tac }}$ promoter of the IMPACT ${ }^{\mathrm{TM}}$ I kit supplied vector $\mathrm{pCYB3}$ was replaced with the promoter from gene 10 of T7 bacteriophage. The T7 promoter was removed from $\mathrm{pET} 23 \mathrm{~d}$ on a $P v u \mathrm{II} / N c o I$ fragment, and inserted into the $M s c \mathrm{I} / N c o$ I sites of $\mathrm{pCYB} 3$ (which also removed the lacI gene) to make the expression vector pIT. The amplified $n f s B$-containing fragment and pIT were digested overnight with $N c o I$ and SapI prior to ligation. The desired clone, pITB (Figure 3.1), was verified by restriction endonuclease analysis and DNA sequencing as described in Section 2.2.10.

\subsubsection{Expression and purification of $N f S B$.}

NfsB was purified with the IMPACT ${ }^{\mathrm{TM}}$ I kit from New England Biolabs. This system uses the intein, a self-cleavable protein splicing element, from the Saccharomyces cerrevisiae VMAI gene, for the affinity purification of native proteins free of any affinity tag. In the presence of thiols (e.g. dithiothreitol) the modified intein undergoes selfcleavage at its $\mathrm{N}$-terminus. As Figure 3.2 illustrates, the chitin binding domain (CBD) from the chitinase A1 protein of Bacillus circulans WL-12 is added to the C-terminus of the intein to allow for affinity purification on a chitin bead column. Thus, the triple fusion (NfsB, intein and CBD) is bound to a chitin bead column, and native $\mathrm{NfsB}$ is cleaved from the fusion after treatment with DTT as follows (refer to Figure 3.2).

An overnight culture $(5 \mathrm{ml})$ of GJ1158, freshly transformed with pITB, was used to inoculate $0.5 \mathrm{~L} \mathrm{LB}-\mathrm{N}$ medium supplemented with ampicillin. The cells were grown to $\mathrm{A}_{600} \approx 0.7-0.8$ at $37^{\circ} \mathrm{C}$ with shaking (about $2.5-3$ hours). For induction, $5 \mathrm{M} \mathrm{NaCl}$ was 
Figure 3.1 (a) NfsB expression vector pITB. See text for details of construction. Approximate location of mutagenesis target region is indicated. (b) Target region and potential mutations after PCR mutagenesis. Bold font denotes wild-type bases and residues and their respective numbers. Bases that were degenerate in the PCR primers (i.e. targets) are underlined, and the base numbers are included below. The inner table indicates the residue change for each possible nucleotide substitution. Bold italics $=$ mutants that retained some nitroreductase activity. Square brackets $=$ mutations that were obtained, but the proteins were not expressed. Parentheses $=$ mutations that were not obtained. 
a.

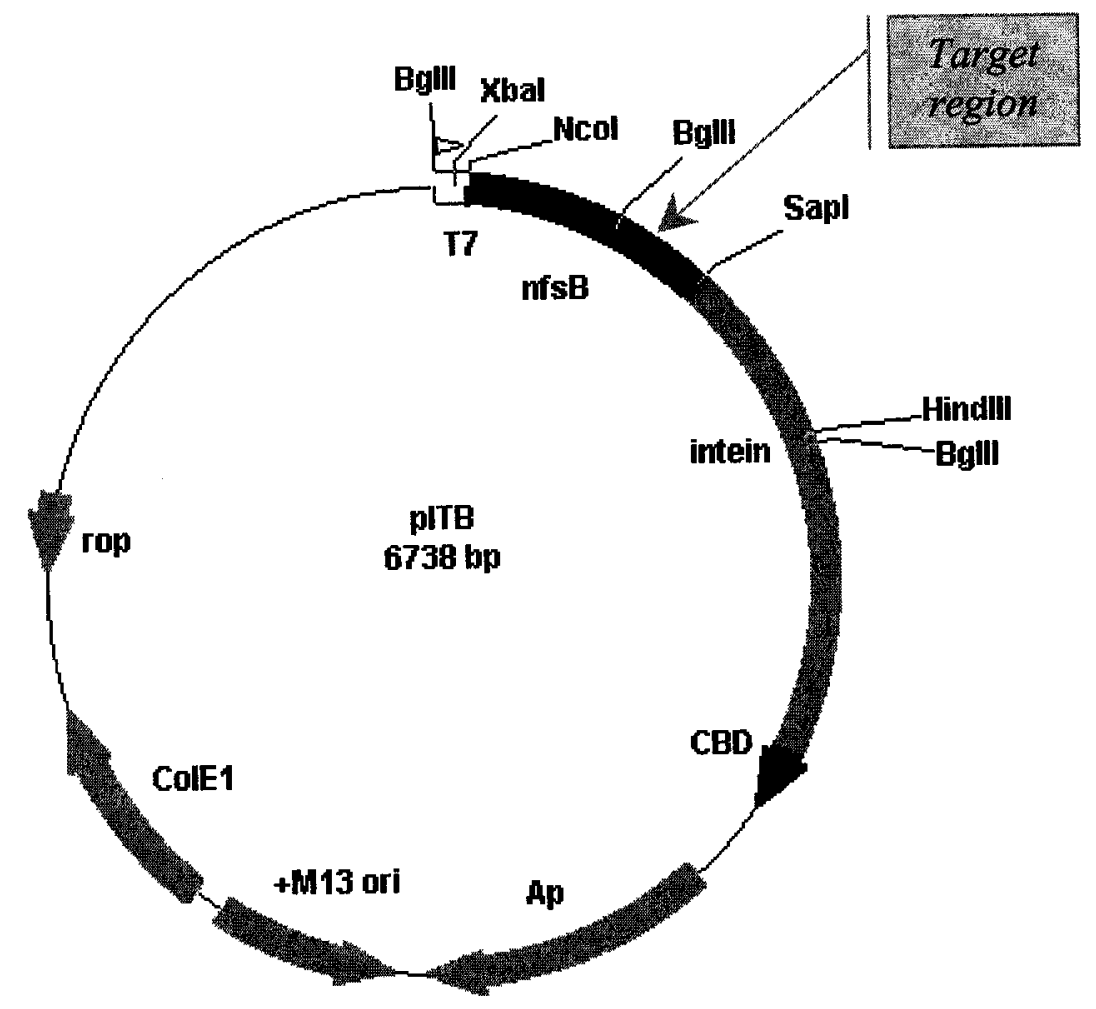

b.

Target

\begin{tabular}{|c|c|c|c|c|c|c|c|}
\hline \multirow[t]{2}{*}{$\begin{array}{l}\text { Nucleotide } \\
\text { substitution }\end{array}$} & $\begin{array}{l}\text { ggc } \\
457\end{array}$ & $\begin{array}{l}\text { gtg } \\
\mathbf{4 6 1}\end{array}$ & $\begin{array}{l}\text { gcg } \\
463 \\
\end{array}$ & $\begin{array}{l}\text { gct } \\
466\end{array}$ & $\begin{array}{l}\text { ctg } \\
470 \\
\end{array}$ & $\begin{array}{l}\text { ggt } \\
472 \\
\end{array}$ & $\begin{array}{l}\text { ctg } \\
476\end{array}$ \\
\hline & $\begin{array}{l}\text { Gly- } \\
153 \\
\end{array}$ & $\begin{array}{l}\text { Val- } \\
154\end{array}$ & $\begin{array}{l}\text { Ala- } \\
155\end{array}$ & $\begin{array}{c}\text { Ala- } \\
156\end{array}$ & $\begin{array}{c}\text { Leu- } \\
157 \\
\end{array}$ & $\begin{array}{l}\text { Gly- } \\
158\end{array}$ & $\begin{array}{c}\text { Leu- } \\
159 \\
\end{array}$ \\
\hline A & Ser & Glu & [Thr] & (Thr) & Gln & Ser & Gln \\
\hline $\mathrm{T}$ & Cys & $\mathrm{X}$ & Ser & (Ser) & $\mathrm{X}$ & [Cys] & X \\
\hline $\mathrm{G}$ & $\mathrm{X}$ & Gly & $\mathrm{X}$ & $X$ & Arg & $\mathrm{X}$ & (Arg) \\
\hline $\mathrm{C}$ & Arg & Ala & Pro & Pro & Pro & (Arg) & (Pro) \\
\hline
\end{tabular}


Figure 3.2 (a) Illustration of the IMPACT I Purification System, adapted from the manufacturer's manual (New England Biolabs). The purification procedure was done at $4^{\circ} \mathrm{C} . \mathrm{MCS}=$ multiple cloning site; $\mathrm{CBD}=$ chitin binding domain. Italics refer to the genetic elements encoding the intein and CBD. (b) Regeneration of the chitin column at room temperature. 
a.

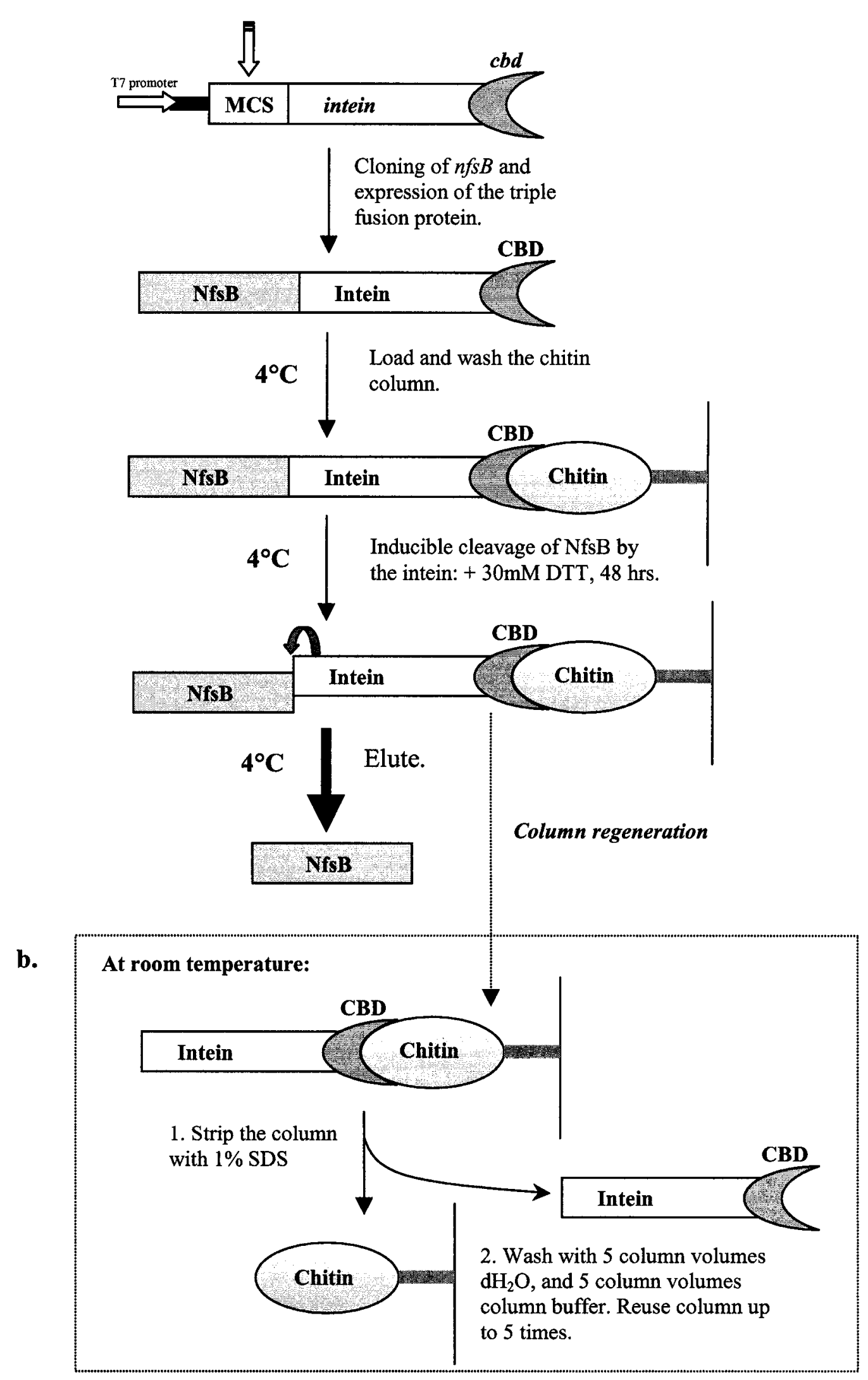


added to a final concentration of $0.3 \mathrm{M}$ and the cells were incubated at $15-20^{\circ} \mathrm{C}$ with gentle shaking for between 6 and 12 hours. The cells were harvested by centrifugation at $5000 \mathrm{x} \mathrm{g}$ and washed with $0.15 \mathrm{M} \mathrm{NaCl}$ (at this stage the pellet could be stored at $-20^{\circ} \mathrm{C}$, if necessary).

The pellet was resuspended in $10 \mathrm{ml}$ column buffer $(20 \mathrm{mM}$ Tris- $\mathrm{HCl} \mathrm{pH} 8.0,500$ $\mathrm{mM} \mathrm{NaCl}, 0.1 \mathrm{mM}$ EDTA) with one Complete ${ }^{\mathrm{TM}}$, Mini, EDTA-free Protease Inhibitor Cocktail Tablet (Roche). The cells were chilled on ice and sonicated with a Virtis VirSonic 60 , power 15 , for $1 \times 30 \mathrm{~s}$ and $2-4 \times 15 \mathrm{~s}$ bursts to yield the clarified extract which was immediately centrifuged at $12000 \mathrm{xg}$ for $30 \mathrm{~min}$ at $4^{\circ} \mathrm{C}$. The clarified extract was promptly loaded onto the chitin bead column.

The following purification procedure was done at $4^{\circ} \mathrm{C}$ (Figure $3.2 \mathrm{a}$ ). Approximately $10 \mathrm{ml}$ of chitin beads were loaded onto a column (Bio-Rad) and equilibrated with $100 \mathrm{ml}$ column buffer. The clarified extract was loaded onto the column and an elution rate of no greater than $0.5 \mathrm{ml} / \mathrm{min}$ was set. The loaded chitin beads were washed with $100 \mathrm{ml}$ column buffer, followed by $50 \mathrm{ml}$ of a high salt buffer (50 mM Tris $\mathrm{pH}$ 8.0, $1 \mathrm{M} \mathrm{NaCl}, 1 \mathrm{mM}$ EDTA), and finally equilibrated with $50 \mathrm{ml}$ cleavage buffer (20 mM Tris- $\mathrm{HCl}$ pH $8.0,50 \mathrm{mM} \mathrm{NaCl}, 0.1 \mathrm{mM}$ EDTA). Cleavage of the fusion protein by the intein was induced by flushing the column with $30 \mathrm{ml}$ cleavage buffer containing $30 \mathrm{mM}$ dithiothreitol (DTT) followed by incubation for 36-48 hours at $4^{\circ} \mathrm{C}$. $\mathrm{NfsB}$ was eluted with $5 \mathrm{ml} 50 \mathrm{mM}$ Tris- $\mathrm{HCl} \mathrm{pH} 7.0$, and stored at $-20^{\circ} \mathrm{C}$ in $40 \%$ glycerol. 
After elution of the protein, 3 column volumes ( $30 \mathrm{ml})$ of stripping buffer (20 $\mathrm{mM}$ Na-Hepes pH 8.0, $500 \mathrm{mM} \mathrm{NaCl}, 1 \% \mathrm{SDS}$ ) were applied (Figure 3.2b). The column was regenerated by washing with 5 column volumes $(50 \mathrm{ml}) \mathrm{ddH}_{2} \mathrm{O}$ followed by $50 \mathrm{ml}$ column buffer. Sufficient column buffer to cover the resin was left in the column, which was then stored at $4^{\circ} \mathrm{C}$. The chitin bead column could be used up to five times following stripping and regeneration of the resin.

\subsubsection{Protein analysis.}

Protein concentrations were determined as described by the manufacturer for the Bio-Rad protein assay with a bovine serum albumin standard curve. Protein products were run on $10 \%$ or $12.5 \%$ SDS-PAGE gels according to standard protocol. The gels were stained for $30 \mathrm{~min}$ in Coomassie Blue (2.5 g Coomassie Brilliant Blue, $450 \mathrm{ml}$

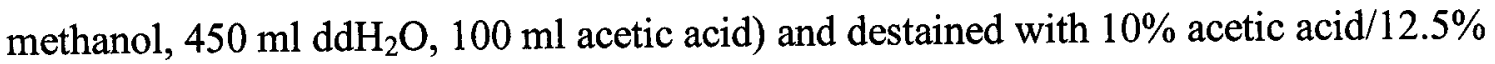
isopropanol.

\subsubsection{PCR mutagenesis.}

The mutational target in NfsB consists of 7 amino acids, G153-L159. The MNU and DMNL primers were degenerate at 7 bases, designed to introduce missense mutations in this region of $n f s B$ (refer to Figure $3.1 \mathrm{~b}$ for all possible missense mutations). Introduction of a mix of nucleotides at the target positions of the oligonucleotides during synthesis allowed a pool of primers with varying numbers of mutations to be created during primer synthesis. At each target position during synthesis the bases were drawn from two sources in equal proportions such that $50 \%$ were from the correct base, and $50 \%$ were from an equal mix of all four bases. Therefore, at each position there was a 
$5 / 8$ chance of having the wild-type base inserted. The fraction of oligonucleotides expected to contain a single mutation was calculated using the formula: $n ! P^{n-r}(1-P)^{r} /(n-$ r)! $r$ ! where $n$ is the number of bases in the target sequence to be mutated (in this case 7), $P$ is the probability of any target nucleotide being unchanged (here, $5 / 8$ or 0.625 ), and $r$ is the number of mutations per oligonucleotide (ideally $\mathrm{r}=1$ ) (Sambrook et al., 1989).

Figure 3.3 illustrates the PCR mutagenesis approach, which involved two sequential reactions. The first round of PCR consisted of one reaction for each of the two primer pairs: MNU + ILH, and IMPBU + DMNL. Each reaction $(100 \mu l)$ contained Taq reaction buffer (Gibco BRL), $2 \mathrm{mM} \mathrm{MgCl}, 300 \mathrm{nM}$ of the appropriate primers, $0.2 \mathrm{mM}$ of each deoxynucleoside triphosphate, $2 \mathrm{U}$ Taq DNA polymerase (Gibco BRL) and approximately $50 \mathrm{ng}$ pITB as template. Amplification (program \#1) was achieved through 30 repetitions of a cycle of denaturation for $20 \mathrm{~s}$ at $94^{\circ} \mathrm{C}$, annealing for $30 \mathrm{~s}$ at $50^{\circ} \mathrm{C}$, and elongation for $45 \mathrm{~s}$ at $72^{\circ} \mathrm{C}$. A preincubation at $94^{\circ} \mathrm{C}$ for $2 \mathrm{~min}$ and a postincubation at $72^{\circ} \mathrm{C}$ for 5 min were included. The second round of PCR used the amplification products from the first round as template. This reaction mixture $(100 \mu \mathrm{l})$ included $10 \mu \mathrm{l}$ of amplification product from each first round reaction, $200 \mathrm{nM}$ each of the primers ILH and IMPBU, Taq reaction buffer, $2 \mathrm{mM} \mathrm{MgCl} 2,0.2 \mathrm{mM}$ each $\mathrm{dNTP}$ and 2 U Taq DNA polymerase. The amplification cycle (program \#2) was the same as for the first round, except that the elongation time was extended to $1.5 \mathrm{~min}$, and it was repeated only 15 times. The PCR product $(12 \mu \mathrm{l})$ was run on a $0.7 \%$ agarose gel in the presence of ethidium bromide and the $\sim 1.2 \mathrm{~kb}$ fragment was excised and purified using a GeneClean kit. This product was freshly amplified immediately prior to directly cloning it into 


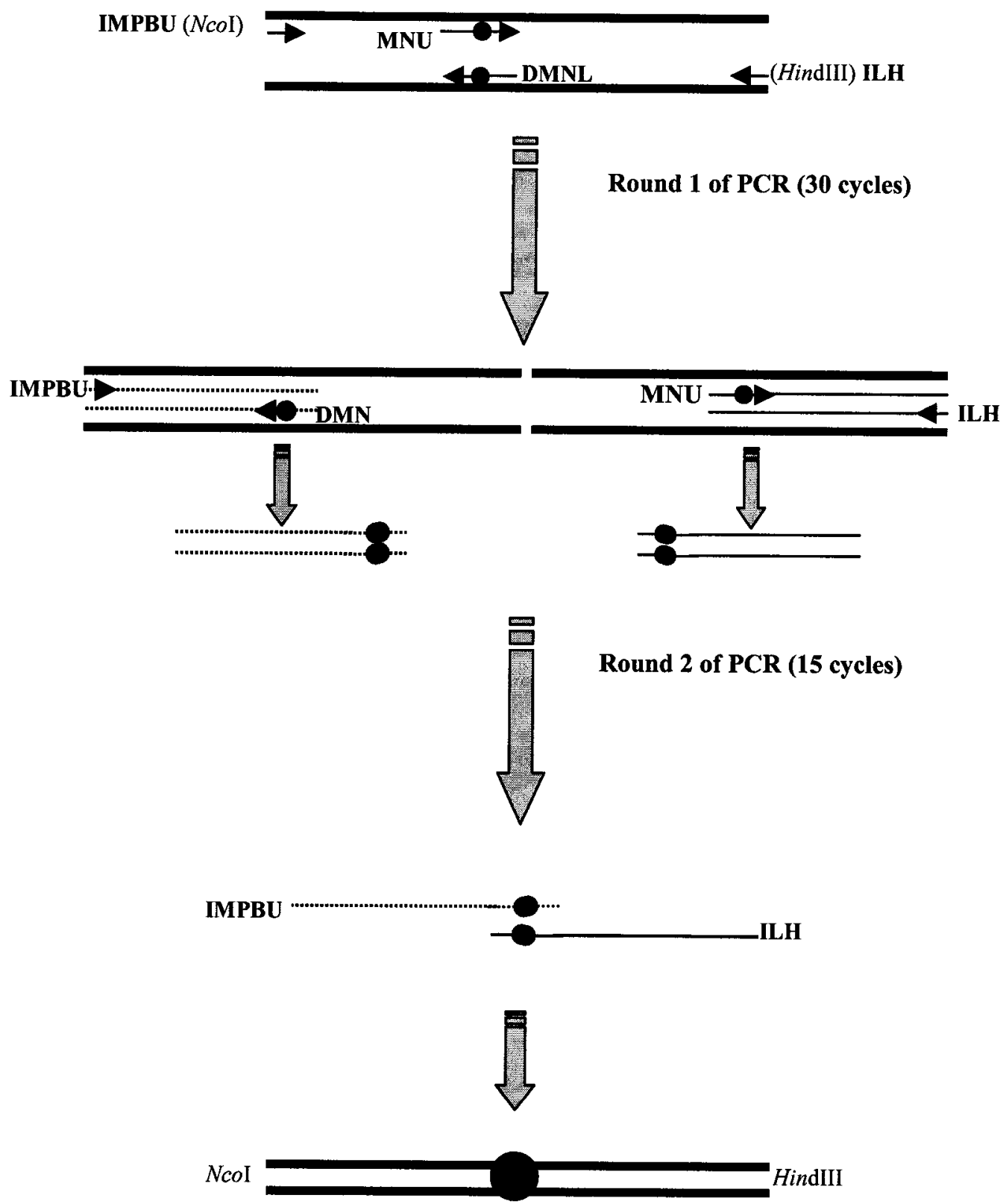

Figure 3.3 Targeted PCR mutagenesis using degenerate primers. Lowercase italics indicate restriction enzyme recognition sites; bold capitals refer to the primers (see Table 3.1) used for amplification at these steps. The ' $\bullet$ ' represents the mutation(s). 
$\mathrm{pCR}^{\circledR} 2.1$ (explained below). The reaction mixture $(100 \mu \mathrm{l})$ contained Taq reaction buffer, $2 \mathrm{mM} \mathrm{MgCl}_{2}, 0.2 \mathrm{mM}$ each dNTP, 2 U Taq DNA polymerase, $600 \mathrm{nM}$ each of the primers IMPBU and ILH, and 1/100 of the PCR product. Amplification was achieved by program \#2 described above.

Direct cloning of PCR amplification products was facilitated by the Original TA Cloning ${ }^{\circledR}$ Kit (Invitrogen). The kit supplied vector, $\mathrm{pCR}^{\circledR} 2.1$, is linearized and contains single 3' thymidine residues. Taq polymerase adds a single deoxyadenosine to the 3' ends of amplification products, so immediate ligation of PCR products into $\mathrm{pCR}^{\circledR} 2.1$ can occur without any restriction enzyme digestion $\operatorname{step}(\mathrm{s}) \cdot \mathrm{pCR}^{\circledR} 2.1$ is a high copy number vector and inserts can be detected using blue/white screening. Here, the freshly amplified PCR product $(1 \mu \mathrm{l})$ was ligated with $\mathrm{pCR}^{\circledR} 2.1(8 \mathrm{ng})$ according to the manufacturer's directions. Transformed DH5 $\alpha$ was plated on LBA+Ap+Xgal and plasmids extracted from white colonies were screened for the presence of the $\sim 1.2 \mathrm{~kb}$ insert by digestion with $E c o$ RI. The target region of each plasmid with an insert was sequenced with primer ET2. If the target contained a single mutation, the whole $n f_{s} B$ gene was sequenced to verify that no other mutation(s) had been introduced.

\subsubsection{Subcloning of selected mutations.}

Plasmids containing unique mutations within the target site (including some with two and three mutations) were subcloned into the Ncol/HindIII sites of pIT, and the constructs were used to transform the host strain GJ1158. One mutant, VAFS, has two mutations, T209 $\rightarrow \mathrm{C}$ and $\mathrm{T} 461 \rightarrow \mathrm{C}$, of which the former is located beyond the target 
region. After insertion into pIT, each mutation of VAFS was subcloned as described below (refer to Figure 3.1a for restriction site locations).

\subsubsection{V154A}

The T461 $\rightarrow$ C mutation of VAFS translates to a Val154 $\rightarrow$ Ala substitution in NfsB. To subclone this mutation, the $X b a \mathrm{I} /$ HindIII fragment of $\mathrm{pITB}$, which contains the complete $n f_{s} B$ gene, was inserted into pUC128 to create pUCIB. The $B g l \mathrm{II} / H$ indIII fragment of pUCIB was then replaced by that of pITVAFS to make the intermediate construct pUCV154A, which contained only the T461 $\rightarrow$ C mutation. The V154A expression construct, pITV154A, was obtained by inserting the $N$ coI/HindIII fragment of pUCV154A into pIT.

\subsubsection{F70S}

The T209 $\rightarrow$ C substitution of VAFS yields a Phe $70 \rightarrow$ Ser mutation in NfsB. Each attempt to make an expression construct of F70S according to the cloning scheme described for V154A (Section 3.2.8.1) failed, resulting in a truncated or mutated $n f s B$ gene. An expression construct of F70S was finally obtained by making an intermediate clone that contained the $n f s B$ gene in the opposite orientation. First, the $X b a \mathrm{I} / H$ ind III fragment of pITVAFS was inserted into pUC128 to make pUCVAFS. Ligating the $X b a \mathrm{I} / B g l \mathrm{II}$ fragment of pUCVAFS and the $B g / \mathrm{II} / H i n d \mathrm{III}$ fragment of pITB in a single reaction with the vector pNEB193 made the intermediate clone pNEBF70S. The pNEB193 vector contains the identical multiple cloning site as pUC128 but in the opposite orientation. Finally, the NcoI/HindIII fragment of pNEBF70S was subcloned into pIT to create the expression construct pITF70S. 


\subsubsection{Confirmation of protein expression and nitroreductase activity}

A small scale culture of each mutant was grown and the cells were run on a $10 \%$ SDS-PAGE gel to ensure that the triple fusion protein was expressed. LB-N $(10 \mathrm{ml})$ with ampicillin was inoculated with $100 \mu \mathrm{l}$ of an overnight culture and grown at $37^{\circ} \mathrm{C}$ for 2.5 hours. The culture was divided into two tubes and one was induced with $0.3 \mathrm{M} \mathrm{NaCl}$; both tubes were gently shaken at $20^{\circ} \mathrm{C}$ for 5 hours. A $0.5 \mathrm{ml}$ sample was removed from each tube, centrifuged, and the pellets were resuspended in $25 \mu$ SDS-PAGE gel loading buffer (50 mM Tris- $\mathrm{HCl} \mathrm{pH} 6.8,2 \% \mathrm{SDS}, 0.01 \%$ bromophenol blue, 10\% glycerol) of which 5-10 $\mu$ l was loaded on a 10\% SDS-PAGE gel. The remaining culture was centrifuged and the pellet was used to make cell extracts according to the procedure described in Section 2.2.4. Nitroreductase activity was assayed as nitrofurazone reductase activity (Section 2.2.6).

\subsubsection{Intein activity}

Cleavage of fusion protein was induced in cell extracts of the mutants to ensure that the intein was functional. DTT $(50 \mathrm{mM})$ was added to the $100 \mu \mathrm{l}$ cell extract, which was then incubated at $4^{\circ} \mathrm{C}$ for 36 hours; cell extract/DTT mixture $(10 \mu 1)$ was run on a $10 \%$ SDS-PAGE gel to confirm the presence of a band corresponding to NfsB (approximately $27 \mathrm{kD}$ ) in the induced fraction.

\subsubsection{Fluorescence spectroscopy}

Fluorescence spectra of wild-type NfsB and the mutants VAFS, F70S, V154A, G158S, G153S and A156P were acquired at room temperature on a Hitachi F-2000 fluorescence spectrophotometer. The purified protein samples were adjusted to $1 \mu \mathrm{M}$ in 
$50 \mathrm{mM}$ Tris- $\mathrm{HCl} \mathrm{pH} 7.0$ buffer, except for mutant A156P for which only a $0.5 \mu \mathrm{M}$ sample was used due to low yields of purified protein. Tryptophan residues were specifically excited at $290 \mathrm{~nm}$ and emission spectra were recorded from 300 to $450 \mathrm{~nm}$. FMN was excited at $464 \mathrm{~nm}$, an optimal wavelength for excitation as determined from an excitation scan (emission $525 \mathrm{~nm}$ ) of $300-800 \mathrm{~nm}$, and emission spectra were recorded from $500-600 \mathrm{~nm}$. The fluorescence emitted by the buffer was subtracted from all spectra.

\subsubsection{Ultraviolet spectroscopy}

Samples $(0.1 \mu \mathrm{M})$ from the same protein preparations analyzed for fluorescence were used to measure tryptophan and tyrosine absorption $(280 \mathrm{~nm})$ on a Pharmacia Biotech Ultrospec 3000 UV/Visible spectrophotometer.

\subsubsection{Enzyme kinetics}

Nitroreductase activity was measured as nitrofurazone reductase on a Dynatech Model MR5000 microplate reader. The reduction of nitrofurazone was measured as a decrease in absorption at $405 \mathrm{~nm}$ (molar extinction coefficient $11,100 \mathrm{M}^{-1} \mathrm{~cm}^{-1}$ ) at $23^{\circ} \mathrm{C}$. The reaction mixture $(195 \mu \mathrm{l})$ contained $50 \mathrm{mM}$ Tris $-\mathrm{HCl}(\mathrm{pH} 7.0), 0.2 \mu \mathrm{g}$ purified enzyme, and appropriate concentrations of nitrofurazone and NADPH or NADH. Nitrofurazone and $\mathrm{NAD}(\mathrm{P}) \mathrm{H}$ concentrations were determined spectrophotometrically (NAD(P)H molar extinction coefficient $6,220 \mathrm{M}^{-1} \mathrm{~cm}^{-1}$ at $340 \mathrm{~nm}$ ). For each set of reactions, the kinetic parameters were measured for 5 concentrations of nitrofurazone each at 6-8 concentrations of NAD(P)H. Each strain was analyzed at least four times for each cofactor, with at least two independent enzyme preparations, and on separate days. 
The reaction velocities were measured at $10 \mathrm{~s}$ intervals for 45 readings, and the raw data was stored as an ASCII file by the Biolinx 2.0 program (Dynatech Laboratories, Inc.). The reaction rate data were calculated by linear regression using the Microplate Analysis (MPA) Program (Brooks, 1994). This program uses an absorbance factor to determine the enzyme velocity of each reaction. The absorbance factor is calculated as follows:

$$
\text { absorbance factor }=1000 \times \mathrm{ABS}_{\mathrm{STD}} \times 60 /\left(\mathrm{A} \times \varepsilon \times l_{\mathrm{STD}}\right)=\mathrm{nmol} / \mathrm{min}(\mathrm{mU})
$$

The absorbance of the standard, $\mathrm{ABS}_{\mathrm{STD}}$, was determined from six independent absorbance measurements on an independently calibrated Pharmacia Biotech Ultrospec $3000 \mathrm{UV} /$ Visible spectrophotometer (not a microplate reader).

$$
\mathrm{ABS}_{\mathrm{STD}}=\underline{\mathbf{0 . 7 4 5}}=\text { absorbance of the standard }(67.1 \mu \mathrm{M} \text { nitrofurazone) (2) }
$$

The machine (microplate reader) slope, $\mathrm{A}$, was determined by plotting the machine absorbance versus microplate well volume $(\mu \mathrm{l})(50,100,150,200,250,300 \mu \mathrm{l}$ well volumes were used), and calculating the slope.

$$
\mathrm{A}=\underline{\mathbf{0 . 0 0 2 6}} \mathrm{ABS} / \mu \mathrm{l}=\text { machine slope }(3)
$$

The molar extinction coefficient of nitrofurazone at $405 \mathrm{~nm}$ was determined on the independently calibrated spectrophotometer.

$$
\varepsilon=\underline{11100} \mathrm{M}^{-1} \mathrm{~cm}^{-1}=\text { molar extinction coefficient of nitrofurazone }(405 \mathrm{~nm})(4)
$$

The pathlength, $l_{\mathrm{STD}}$, is $1.0 \mathrm{~cm}$, and the values of 60 and 1000 are present to convert the result into minutes and nmol, respectively. Therefore, substitution of the values 
calculated in equations 2-4, along with $l_{\mathrm{STD}}$ and the conversion factors into equation (1) yields an absorbance factor of $1548.86 \mathrm{nmol} / \mathrm{min}$.

absorbance factor $=1000 \times 0.745 \times 60 /(0.0026 \times 11100 \times 1.0)=\underline{\mathbf{1 5 4 8 . 8 6}}$

$\mathrm{nmol} / \min (5)$

The enzyme velocity data were saved as a Kinetics file, imported into the Kinetics Program (Brooks, 1992) and used to calculate the kinetic parameters $\mathrm{Km}^{\text {app }}$ and Vmax ${ }^{\text {app }}$ for $\mathrm{NAD}(\mathrm{P}) \mathrm{H}$. Calculation of the kinetic parameters for nitrofurazone was achieved by reentering the same data sets with nitrofurazone concentrations as the variable substrate. The enzyme activity curves and Lineweaver-Burk plots were viewed in the Kinetics Program, but in order to print the curves the data was reentered into Microsoft ${ }^{\circledR}$ Excel. Double reciprocal plots of substrate (nitrofurazone, NADH or NADPH) against enzyme velocity were plotted, but as the reactions were not conducted under saturating conditions, replots of the apparent kinetic parameters $\left(\mathrm{Km}^{\mathrm{app}}, \mathrm{Vmax}{ }^{\mathrm{app}}\right)$ derived from these primary double reciprocal plots were used to determine the actual $\mathrm{Km}$ and Vmax values. Diagrams of the primary double reciprocal plots (Figure 3.4a) and of the secondary replots (Figure $3.4 \mathrm{~b}, \mathrm{c}$ ) illustrate this method for the graphical determination of kinetic parameters as described in Copeland (2000).

The $\mathrm{Km}$ values for nitrofurazone and $\mathrm{NAD}(\mathrm{P}) \mathrm{H}$ were determined from their respective Vmax ${ }^{\text {app }}$ reciprocal plots. The actual Vmax value was taken as an average of the two plots (i.e. nitrofurazone and NAD(P)H Vmax values). The turnover number, $\mathrm{k}_{\text {cat }}$, was calculated as: $\operatorname{Vmax} / E(t)$ where $E(t)$ is the total amount of enzyme in the reaction (in moles). The errors were calculated based on regression analysis $\left(R^{2}\right.$ value) of the 
a)

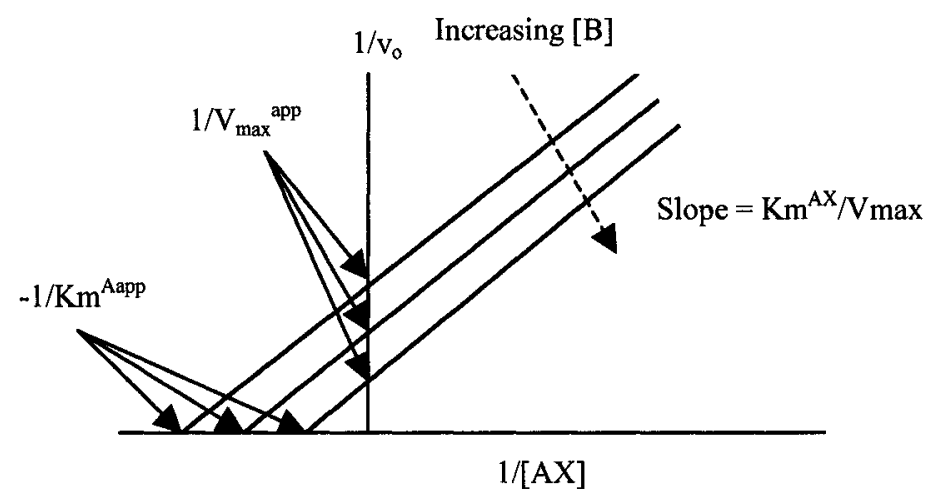

b)

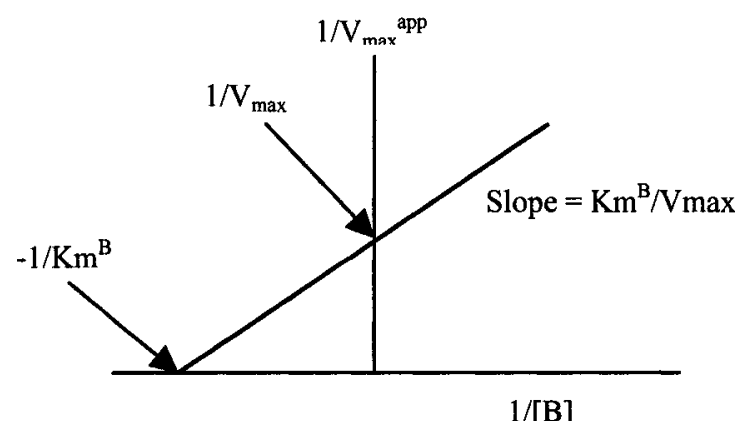

c)

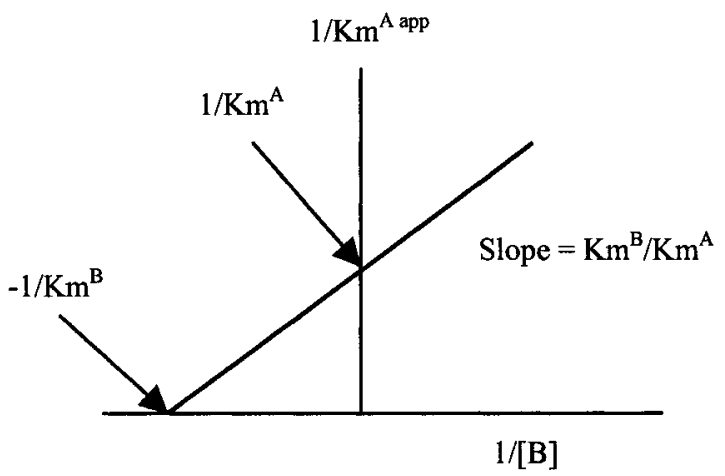

Figure 3.4 (a) Double reciprocal plot for a ping-pong Bi Bi reaction (adapted from Copeland, 2000). Replots as (b) $1 / \mathrm{Vmax}{ }^{\text {app }}$ versus $1 /[\mathrm{B}]$ and (c) $1 / \mathrm{Km}^{\mathrm{AX}}$ app versus $1 /[\mathrm{B}]$, illustrating the graphical determination of $\mathrm{Km}^{\mathrm{AX}}, \mathrm{Km}^{\mathrm{B}}$, and Vmax for a ping-pong $\mathrm{Bi} \mathrm{Bi}$ reaction (adapted from Copeland, 2000). 
secondary plots. Standard error analysis rules were used when combining these errors in further calculations (ex. $\mathrm{k}_{\mathrm{cat}} / \mathrm{Km}$ values).

\subsubsection{Structural analysis}

Three dimensional protein structures were downloaded as PDB files from the Protein Databank (www.rcsb.org) and viewed with Swiss-PdbViewer v.3.7b2, obtained from http://www.expasy.ch/spdbv (Guex and Peitsch, 1997). Distances between atoms, bond lengths and structural alterations after residue changes were obtained with this program. A more comprehensive analysis of these structures is the focus of Chapter 4. 


\section{$\underline{\text { 3.3 Results }}$}

\subsubsection{Expression and purification of $\mathrm{Nfs} B$.}

The key challenge in developing a purification scheme for NfsB is its tendency to aggregate and stick to surfaces. Expression at over $25^{\circ} \mathrm{C}$ leads to the formation of inclusion bodies, affinity purification is frustrated by on-column aggregation, and storage of purified NfsB in glass containers with at least $40 \%$ glycerol is necessary otherwise the protein disappears, presumably as it sticks to the container walls. Aggregation of $\mathrm{NfsB}$ was particularly evident during initial attempts to purify it with an N-terminus His-tag (data not shown). The importance of the $\mathrm{N}$-terminus residues for $\mathrm{NfsB}$ function, and the possibility that the His-tag was contributing to protein aggregation, motivated the search for a different purification strategy. The appeal of the IMPACT ${ }^{\mathrm{TMI}}$ kit was the one step purification of native enzyme. Purification of NfsB with the IMPACT ${ }^{\mathrm{TM} I}$ system is not work intensive, but it is time consuming. For instance, cleavage of the fusion protein by the intein is achieved with incubation at $4^{\circ} \mathrm{C}$ for two days. However, pure NfsB without any affinity tags is obtained directly.

The first attempts to purify NfsB with the IMPACT ${ }^{\mathrm{TM}}$ I kit were not successful as the presence of the NfsB fusion protein was not obvious after expression with the $\mathrm{p}_{\text {tac }}$ regulated $n f s B$ construct. To reduce the background of host proteins and increase the expression of NfsB a new construct, pITB (Figure 3.1a), was created such that the triple fusion protein was under the control of the strong gene 10 promoter of bacteriophage T7. Expression of the protein thus relies upon the presence of T7 RNA polymerase (RNAP). Proteins under the control of a T7 promoter are usually expressed in the $E$. coli strain 
BL21(DE3) which encodes T7 RNAP on a lambda prophage under the control of the lacUV5 promoter. Induction of T7 RNAP by IPTG results in the overexpression of the target protein. An alternative to induction by IPTG is possible with GJ1158, a modified strain of BL21(DE3) (Bhandri and Gowrishankar, 1997). In this strain, T7 RNAP is under the transcriptional control of the cis-regulatory elements of the osmoresponsive proU operon, and may be induced by $\mathrm{NaCl}$. The lower cost and toxicity of $\mathrm{NaCl}$ make it an appealing alternative to IPTG. Above $25^{\circ} \mathrm{C}$, most of the fusion protein is localized in the insoluble fraction (see Figure 3.5), presumably due to the formation of inclusion bodies. Therefore, cultures were incubated at $15-20^{\circ} \mathrm{C}$ with gentle shaking for six to twelve hours after the addition of $\mathrm{NaCl}$. Under these conditions, most of the fusion protein was present in the soluble fraction. Reduction of inclusion bodies has been noted previously when GJ1158 is used as an expression host, perhaps due to the intracellular accumulation of glycine betaine, thought to reduce inclusion bodies, that occurs upon osmotic upshock (Bhandri and Gowrishankar, 1997; Blackwell and Horgan, 1991). However, compared to the expression of fusion protein in BL21 induced with IPTG, any increase in solubility provided by GJ1158 appeared negligible for NfsB (data not shown). Initial purification attempts were not successful in obtaining native $\mathrm{NfsB}$ protein, as the protein would mostly elute from the column following denaturation with SDS (i.e. when the column was stripped) (Figure 3.6). This was likely due to on-column aggregation of the protein, which can occur when too much protein is loaded onto an inadequate amount of resin. Therefore, less cellular extract was loaded, and greater column bed volumes were used in subsequent purification attempts. Purification of NfsB 


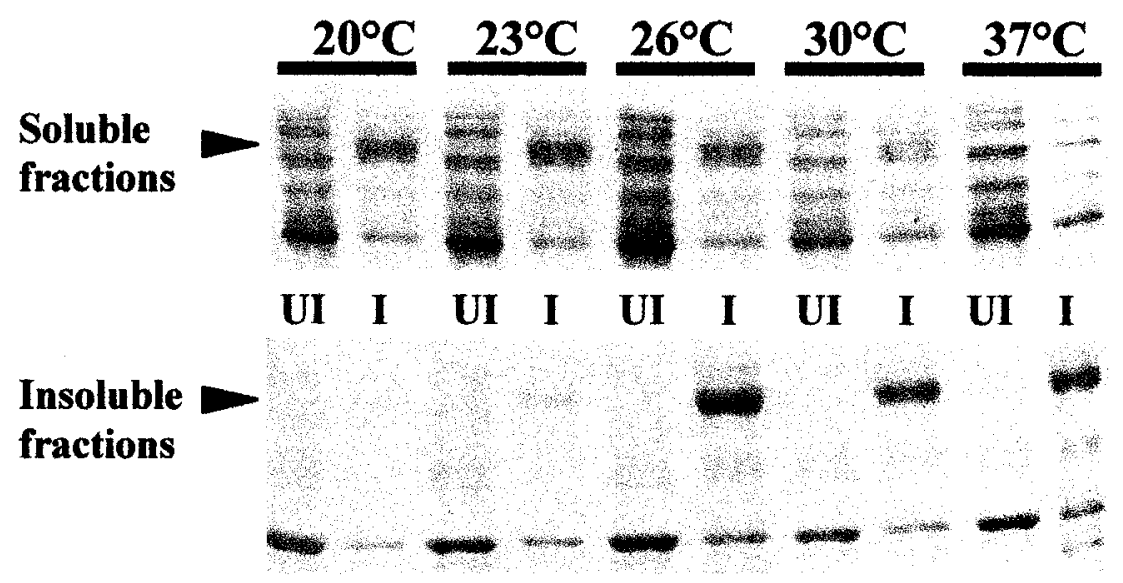

Figure 3.5 Expression of the $\mathrm{NfsB}$ triple fusion protein at a range of temperatures. At elevated temperatures the protein is increasingly localized within the insoluble fraction of the induced crude extracts. Arrows indicate the triple fusion protein with NfsB. UI = uninduced; $\mathrm{I}$ = induced. 


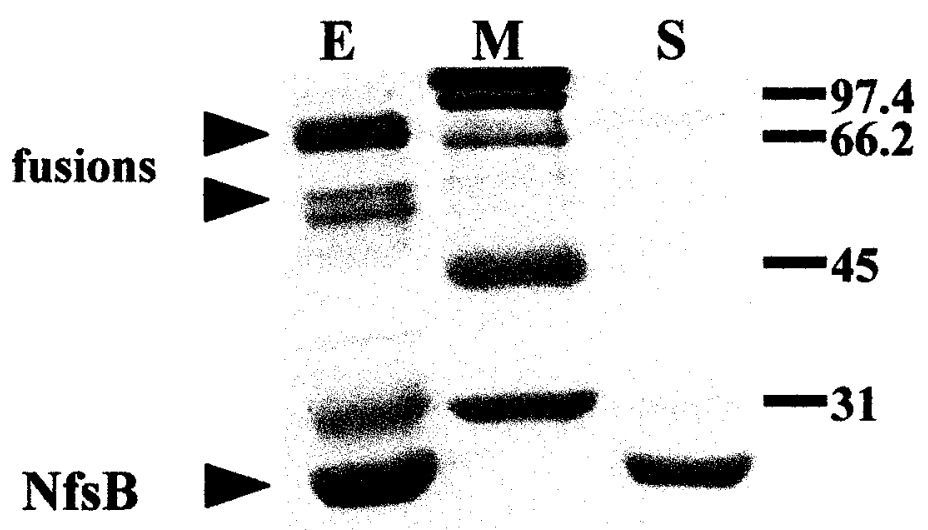

Figure 3.6 Initial $\mathrm{NfsB}$ purification attempt. Lanes: $\mathrm{E}=$ Eluted (purified) fraction; $\mathrm{M}=$ marker (BioRad Broad Range); $S=$ stripped fraction. Arrows point to (from the top) the triple fusion, double fusion and $\mathrm{NfsB}$ proteins. 
was further frustrated by the presence of contaminating bands in the elution fractions (Figure 3.6). Elimination of these contaminants was achieved with an additional wash with a high salt $(1 \mathrm{M} \mathrm{NaCl})$ buffer during the purification process, and by excluding the detergent component (Triton-X 100) of the column buffer. NfsB of high purity (about 25 $\mathrm{mg} / \mathrm{L}$ ) was subsequently obtained (Figure 3.7 ), and stored in glass tubes with $40 \%$ glycerol at $-20^{\circ} \mathrm{C}$. However, purified $\mathrm{NfsB}$ represented less than $1 \%$ of the protein in the soluble fraction of the cellular extract, which was mostly a consequence of the low levels of expression necessary to minimize protein aggregation.

\subsubsection{Characterization of wild-type NfsB}

\subsubsection{NR Activity}

In crude cellular extract, the host strain, GJ1158, exhibited about two-fold more $\mathrm{NADPH}$-associated NR activity $(42.3 \mathrm{nmol} / \mathrm{min} / \mathrm{mg})$ than NADH-associated NR activity $(17.9 \mathrm{nmol} / \mathrm{min} / \mathrm{mg}$ ) (Table 3.2). The greater NR activity associated with NADPH is attributable to the existence of NfsA, the major, and NADPH-linked, NR component of E. coli. The presence of vector encoded wild-type NfsB did not yield an increase in NR activity without induction of the NfsB protein. However, following induction the increase in NADPH and NADH associated NR activities were approximately 7- and 18fold, respectively.

\subsubsection{Structural integrity}

Fluorescence spectra are sensitive to changes in the solvent polarity near the fluorophore, which makes this technique a useful tool to evaluate the structural integrity of proteins. In $\mathrm{NfsB}$ there are two primary sources of fluorescence: the indole and phenyl 


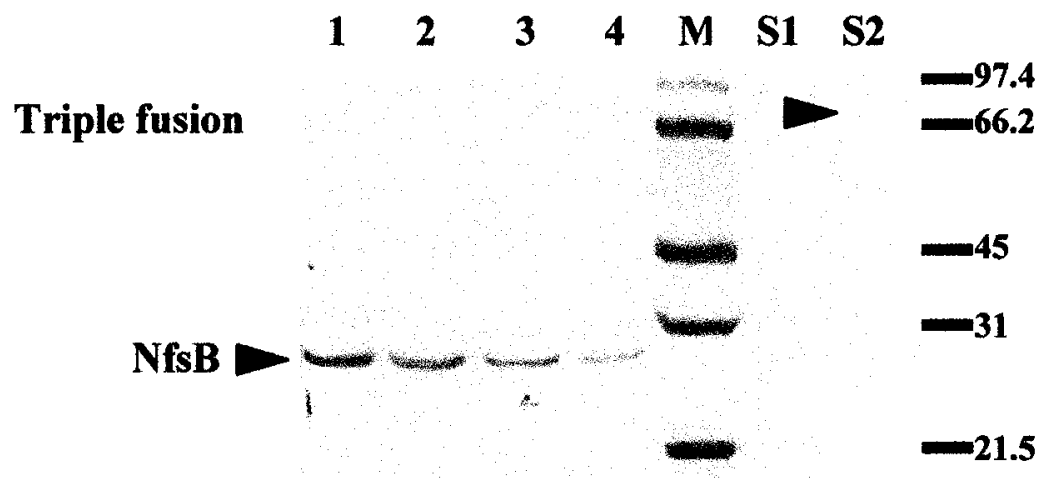

Figure 3.7 Purifed NfsB. Lanes 1-4: eluted fractions; Lane $M$ is the standard marker (BioRad Narrow range); S1, S2 are the stripped fractions (SDS eluent). 
Table 3.2 Nitrofurazone reductase specific activities $(\mathrm{nmol} / \mathrm{min} / \mathrm{mg})$ of cell extracts containing vector encoded wild-type and mutated NfsB.

\begin{tabular}{cccc}
\hline Strain & $\begin{array}{c}\text { Amino acid } \\
\text { mutation }\end{array}$ & NADH & NADPH \\
\hline Wild-type & None & $320.1(48)$ & $290.8(51)$ \\
GJ1158 & None & $17.9(3.3)$ & $42.3(5.1)$ \\
(no vector) & & & \\
G158S $^{\mathrm{a}}$ & Gly158 $\rightarrow$ Ser & $232.8(71)$ & $298.9(65)$ \\
VAFS $^{\mathrm{a}, \mathrm{d}}$ & $\begin{array}{l}\text { Val154 } \rightarrow \text { Ala } \\
\text { Phe70 } \rightarrow \text { Ser }\end{array}$ & $334.0(108)$ & $358.3(121)$ \\
G153S $^{\mathrm{b}}$ & Gly153 $\rightarrow$ Ser & $96.7(25)$ & $142.6(32)$ \\
A156P $^{\mathrm{c}}$ & Ala156 $\rightarrow$ Pro & 17.6 & 44.0 \\
& & & \\
\hline
\end{tabular}

Note: Values averaged from at least 3 trials with the exception of A156P. Average deviations are shown in parentheses.

${ }^{2}$ Mutants with activity that were further characterized.

${ }^{b}$ Mutant had activity but was eventually discarded as enzyme velocity was too low to assay accurately.

'No activity greater than the host strain. Similar values obtained for all mutants with the exceptions listed here.

${ }^{\mathrm{d}}$ The Phe $70 \rightarrow$ Ser mutation is responsible for this mutant's activity. 
fluorophores of the aromatic amino acids (tryptophan and tyrosine residues), and FMN. Excitation at $290 \mathrm{~nm}$ or greater minimizes the contribution of tyrosine residues such that fluorescence emissions from tryptophan can be isolated (Yelamos et al., 1999). Fluorescence spectra of $1 \mu \mathrm{M}$ wild-type NfsB were obtained for both tryptophan and FMN.

NfsB contains three tryptophan residues (W46, W94, W138) that are within approximately $10 \AA$ of one another. W138 is less than $5 \AA$ from bound nicotinic acid, and within $8 \AA$ of FMN and Phe-124, a residue of importance in substrate recognition (Zenno et al., 1996c). Excitation of $\mathrm{NfsB}$ at $290 \mathrm{~nm}$ yields an emission peak at $345 \mathrm{~nm}$ (Table 3.3), which is within the normal range of tryptophan emission peaks (Creighton, 1993). Tryptophan absorbance was measured by UV spectroscopy as well. At $280 \mathrm{~nm}$, $0.1 \mu \mathrm{M}$ of NfsB had an absorbance value of 0.213 (Table 3.3).

An excitation scan of NfsB revealed a peak at $464 \mathrm{~nm}$ (Figure 3.8a) that is within the characteristic range of FMN-containing proteins (Munro and Noble, 1999). Emission scans between 500-600 nm revealed two peaks ascribed to bound FMN in NfsB at 525 $\mathrm{nm}$ (peak I) and $550 \mathrm{~nm}$ (peak II), also within the expected range, while FMN alone had a single peak at $525 \mathrm{~nm}$ (Figure 3.8b). Peak I exhibits greater intensity than peak II (Figure $3.8 \mathrm{~b}, \mathrm{c})$, with a peak I:peak II intensity ratio of 1.3 (Table 3.3 ).

\subsubsection{Enzyme kinetics}

Kinetic analysis can reveal two important aspects of an enzyme's function: kinetic parameters, and evidence concerning the reaction mechanism. However, the determination of initial velocities in two substrate systems is problematic, particularly if 


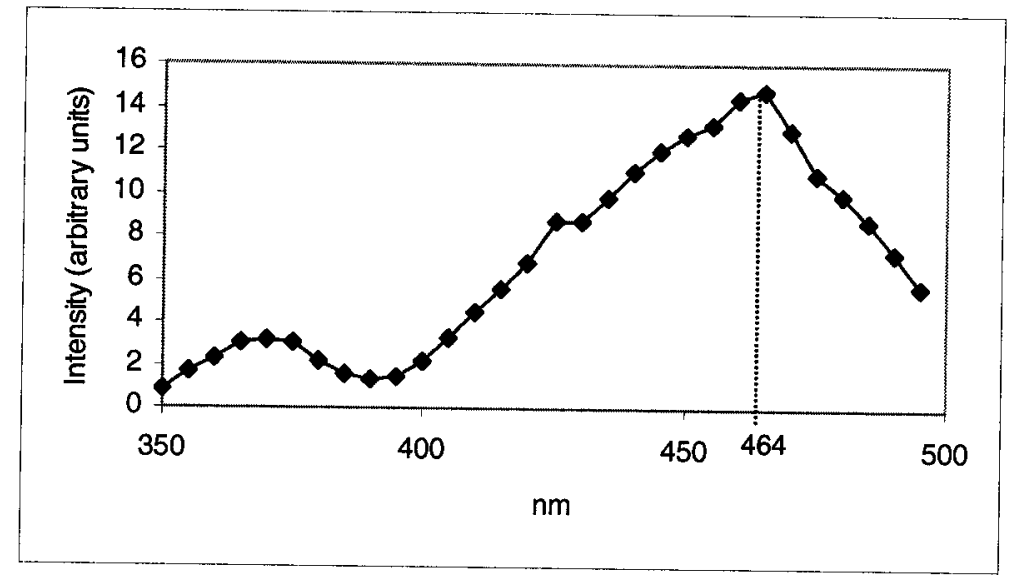

(a)

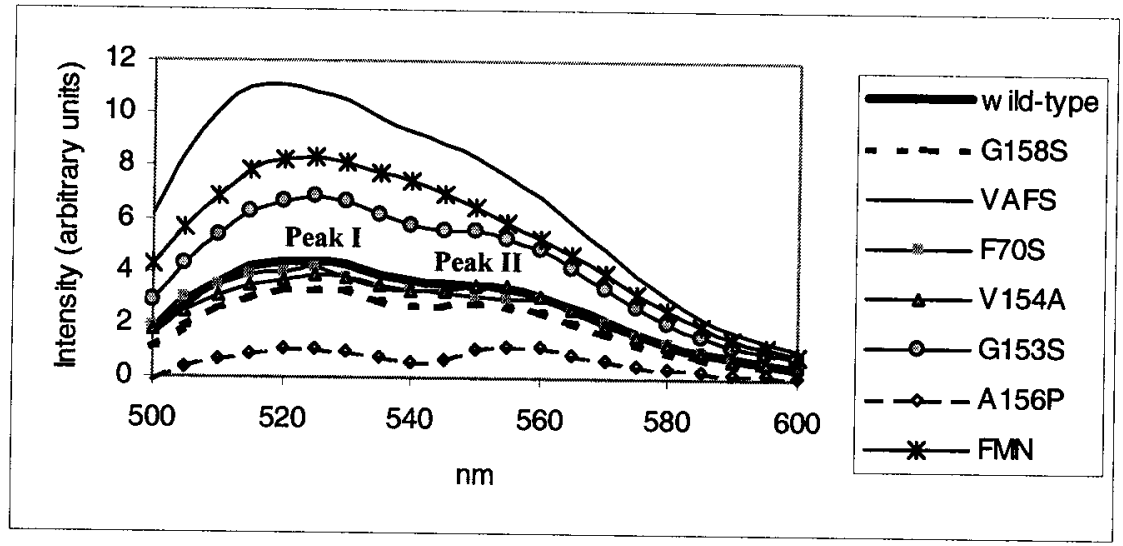

(b)

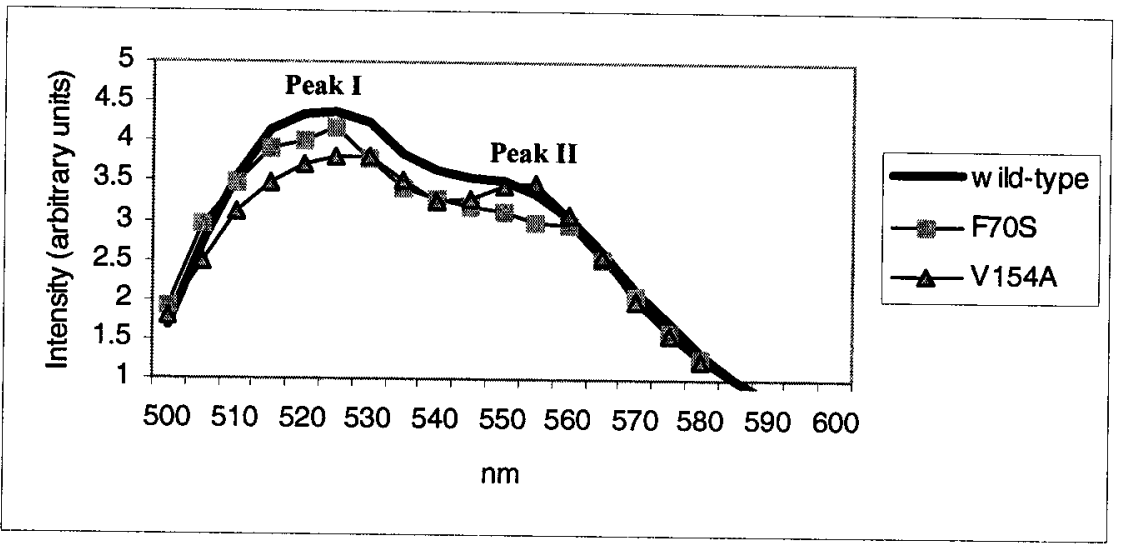

(c)

Figure 3.8 Fluorescence spectra. (a) Wild-type excitation scan, emission at $525 \mathrm{~nm}$. (b) Emission scans of wild-type NfsB and the mutant enzymes, excitation at $464 \mathrm{~nm}$. Peak I and peak II are identified (see text for details). (c) A closer view of the wild-type, F70S and V154A emission scans from (b). 
Table 3.3 Fluorescence emission peaks (excitation at $290 \mathrm{~nm}$ and $464 \mathrm{~nm}$ ) and UV absorbance values $(280 \mathrm{~nm})$ for wild-type and mutant $\mathrm{NfsB}$ proteins.

\begin{tabular}{|c|c|c|c|c|c|c|}
\hline \multirow[t]{2}{*}{ Strain } & \multirow{2}{*}{$\begin{array}{l}\mathrm{NR}^{\mathrm{a}} \\
\text { activity }\end{array}$} & \multirow{2}{*}{$\begin{array}{l}U^{b} \\
280 \mathrm{~nm}\end{array}$} & \multirow{2}{*}{$\begin{array}{l}\operatorname{Trp}^{c} \\
\text { Peak }(n m)\end{array}$} & \multicolumn{3}{|c|}{ FMN $^{\mathrm{d}}$ ex. $464 \mathrm{~nm}$} \\
\hline & & & & Peak (nm) & Intensity & ratio ${ }^{\mathrm{e}}$ \\
\hline FMN & N/A & N/A & N/A & 525 & 50.0 & N/A \\
\hline Wild-type & Full & 0.213 & 345 & $\begin{array}{l}525 \text { (I) } \\
550 \text { (II) }\end{array}$ & $\begin{array}{l}4.4 \\
3.5\end{array}$ & 1.3 \\
\hline F70S & Full & 0.220 & 345 & 520 & 4.0 & 1.3 \\
\hline VAFS & Full & 0.202 & 345 & 520 & 11.2 & N/A \\
\hline G158S & Partial & 0.386 & 375 & $\begin{array}{l}525 \\
550\end{array}$ & $\begin{array}{l}3.3 \\
2.8\end{array}$ & 1.2 \\
\hline G153S & Partial & 0.504 & 375 & $\begin{array}{l}525 \\
550\end{array}$ & $\begin{array}{l}6.9 \\
5.6\end{array}$ & 1.2 \\
\hline V154A & None & 0.604 & 375 & $\begin{array}{l}530 \\
555\end{array}$ & $\begin{array}{l}3.8 \\
3.5\end{array}$ & 1.1 \\
\hline $\mathrm{A} 156 \mathrm{P}$ & None & 0.508 & 375 & $\begin{array}{l}525 \\
555\end{array}$ & $\begin{array}{l}1.1 \\
1.2\end{array}$ & 0.9 \\
\hline
\end{tabular}

${ }^{\mathrm{a}} \mathrm{NR}=$ nitroreductase activity relative to wild-type. Refer to Tables $3.2 \& 3.4$ for actual NR activities.

${ }^{\mathrm{b}}$ Absorbance $(\lambda=280 \mathrm{~nm})$ of $0.1 \mu \mathrm{M}$ protein as determined by Bradford assay.

${ }^{\mathrm{c}}$ Tryptophan specifically excited at $290 \mathrm{~nm}$, emission recorded 300-450 nm. $1 \mu \mathrm{M}$ protein used for measurements except for A156P $(0.5 \mu \mathrm{M})$.

${ }^{d}$ FMN specifically excited at $464 \mathrm{~nm}$, emission recorded $500-600 \mathrm{~nm}$. Fluorescence due to buffer components is subtracted.

${ }^{\mathrm{e}}$ ratio of the intensities of Peak I $(\sim 525 \mathrm{~nm})$ :Peak II $(\sim 550 \mathrm{~nm})$ 
the $\mathrm{Km}$ of the measured substrate is so high that saturation cannot be achieved and Michaelis-Menten single substrate kinetics is not approximated. The NR assay used in this study measures the reduction of nitrofurazone at $405 \mathrm{~nm}$, which introduces a limitation to the maximum concentration of nitrofurazone $(132 \mu \mathrm{M})$ that can be used in a reaction due to the spectrophotometer's sensitivity limit (absorbance of approximately 1). As the $\mathrm{Km}$ of nitrofurazone is greater than $132 \mu \mathrm{M}$, the saturating conditions necessary to mimic single substrate Michaelis-Menten enzyme kinetics could not be met. The solution to this limitation is to use a matrix of substrate concentrations such that at least 25 velocity measurements are made in a single experimental trial (Allison and Purich, 1979). A double reciprocal plot of initial velocity versus the variable substrate concentration (here, NADH or NADPH) is made for at least 4 fixed concentrations of the other, fixed variable substrate (here, nitrofurazone). This primary double reciprocal plot reflects the putative reaction mechanism of the enzyme, as described below in Section 3.3.2.3.1. As well, the values of $-1 / \mathrm{Km}^{\text {app }}$ and $1 / \mathrm{Vmax}^{\text {app }}$ are derived from the $\mathrm{x}$ and $\mathrm{y}$ intercepts, respectively, of this plot (see Figure 3.4a). A secondary plot of the intercepts yields estimates for the actual $1 / \mathrm{Vmax}$ and $1 / \mathrm{Km}$ values of the substrates (Figure $3.4 \mathrm{~b}, \mathrm{c}$ ) (Copeland, 2000). Using this approach, the kinetic parameters of $\mathrm{NfsB}$ for nitrofurazone, NADH and NADPH were determined. Enzyme assays were conducted for 6-8 NADH and NADPH concentrations, each at 4-5 nitrofurazone concentrations and the results were analyzed by least squares analysis (Cleland, 1979). 


\subsection{Mechanism}

The double reciprocal plots described previously in section 3.3.2.3 can test the postulated reaction mechanism of an enzyme. Parallel lines of these plots for the fixed variable substrate often correspond to a ping-pong Bi-Bi kinetic mechanism, while converging lines indicate an ordered (also known as a sequential) reaction mechanism (Segel, 1975). Figures 3.9-3.12 illustrate representative primary double reciprocal plots obtained for wild-type NfsB.

The primary double reciprocal plots, $1 /$ Vi versus $1 /$ [substrate], exhibit parallel lines for both NADH (Figure 3.9a) and NADPH (Figure 3.10a) with nitrofurazone as the fixed variable. Likewise, parallel lines are apparent for nitrofurazone with each of NADH (Figure 3.11a) and NADPH (Figure 3.12a) as fixed variables. This suggests that a ping-pong $\mathrm{Bi}-\mathrm{Bi}$ reaction mechanism is consistent with $\mathrm{Nfs} B$ kinetics.

\subsection{Kinetic parameters}

The kinetic parameters, $\mathrm{Km}$ and $\mathrm{k}_{\mathrm{cat}}$, of $\mathrm{NfsB}$ for the cofactors and nitrofurazone are shown in Table 3.4. These were derived from the secondary double reciprocal plots shown in Figures 3.13-3.16 as described in Section 3.3.2.3. The reduction of nitrocompounds by nitroreductase follows a double-displacement, or ping-pong, reaction mechanism (Figure 3.17). As such there are two activities of the enzyme that must be considered during kinetic analysis. First, the transfer of reducing equivalents, in the form of hydrogen atoms, from the $\mathrm{NAD}(\mathrm{P}) \mathrm{H}$ cofactor to the bound $\mathrm{FMN}$; and second, the transfer of electrons from FMN to the nitro-substituted substrate. Thus, analysis of the reaction mechanism requires the determination of two $\mathrm{Km}$ values (Table 3.4). The first is 


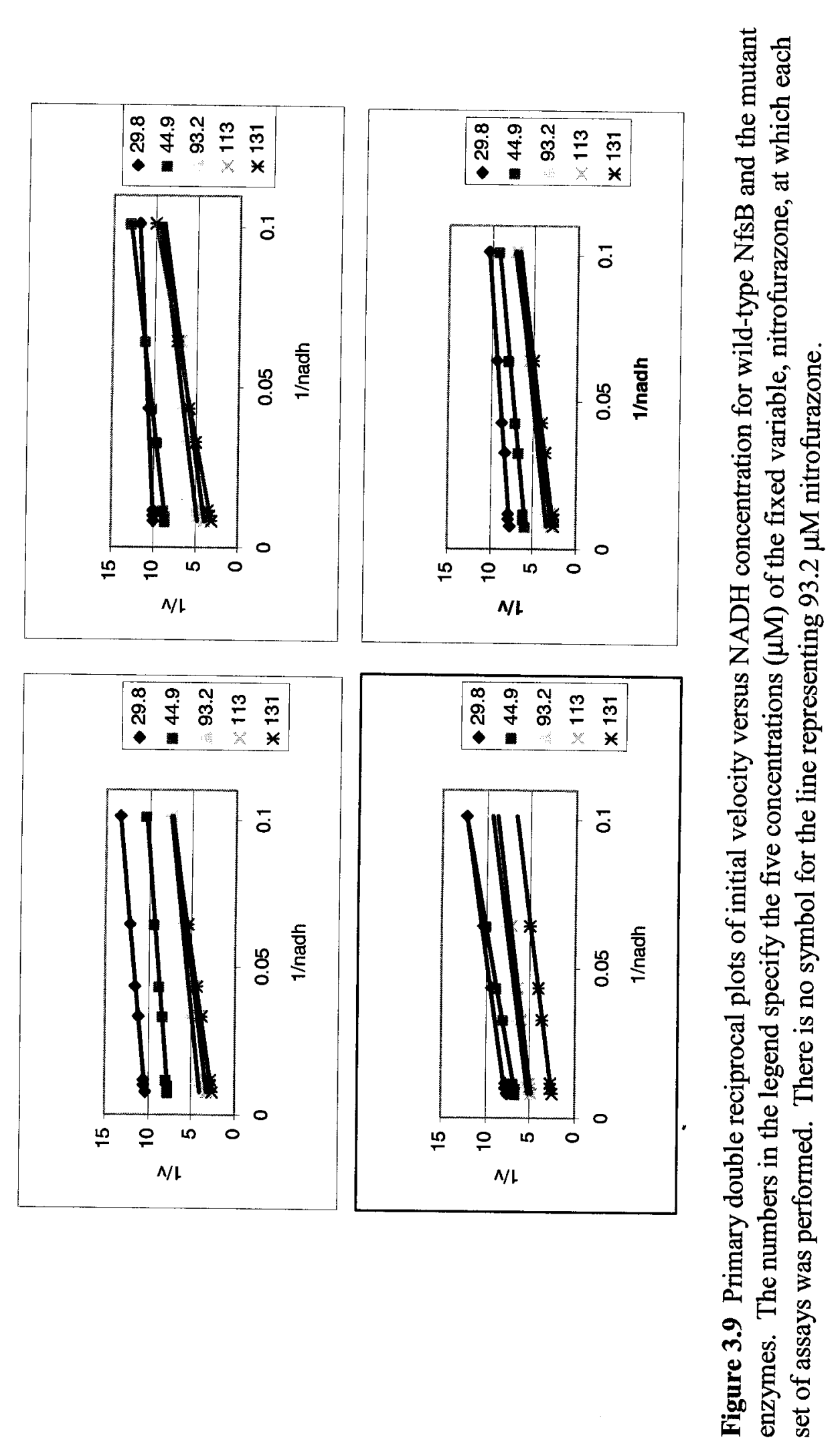


马्8
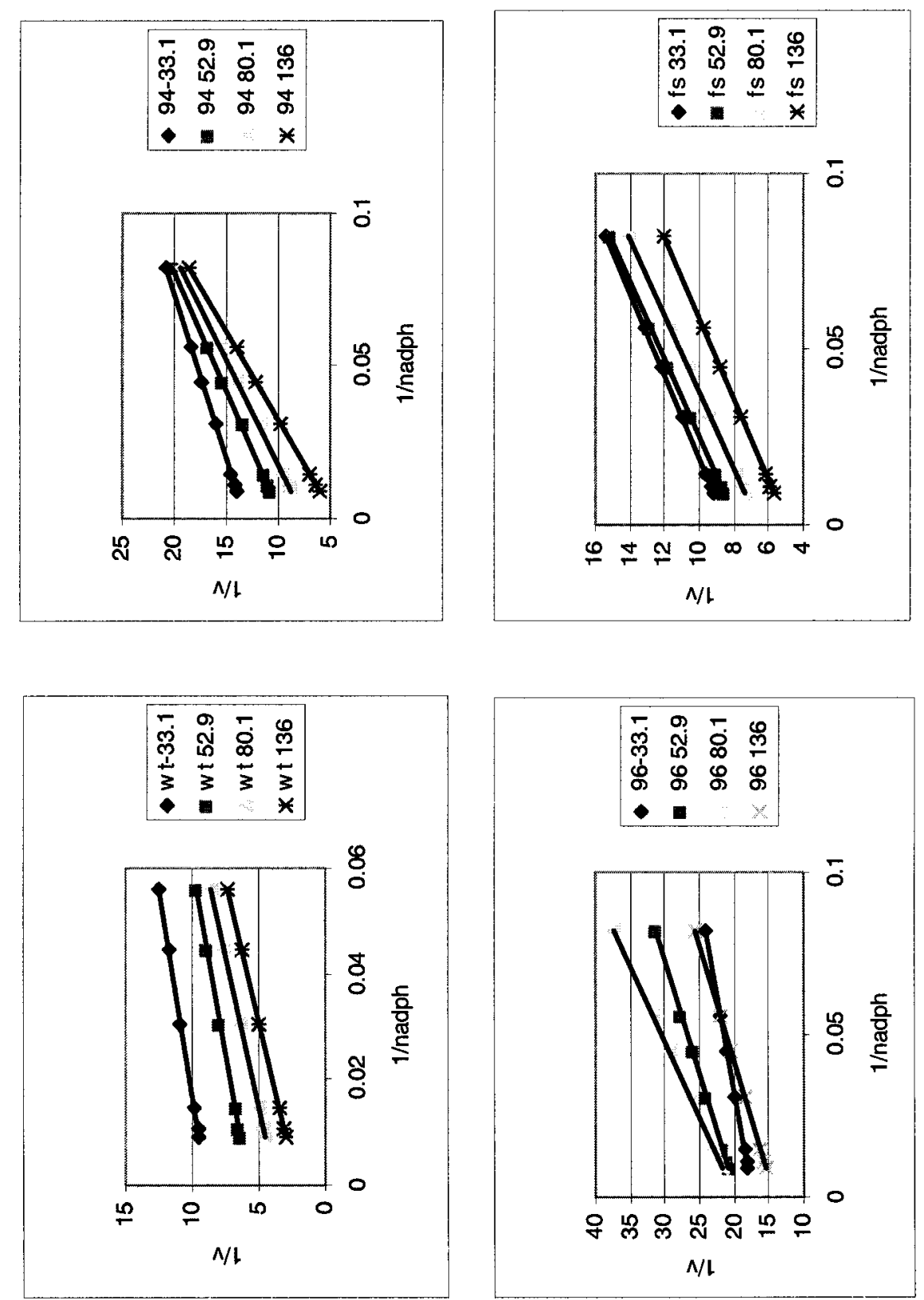

\&

吾

离

त्ञ

定导

3

递

总

要

ষ

용

步

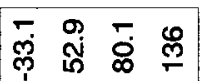

ஸ

롱

忿些

.

를 원

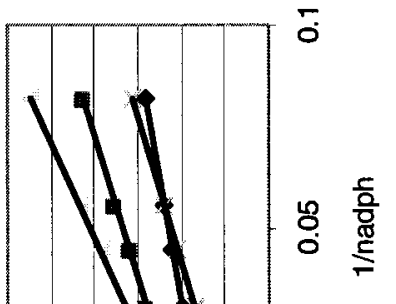

○

乐 $\frac{0}{0} \equiv$

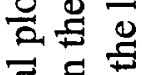

정

氨造

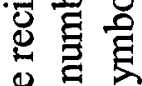

을옹

守焉

寻它

용혀

을처음

莣导

串㤩 

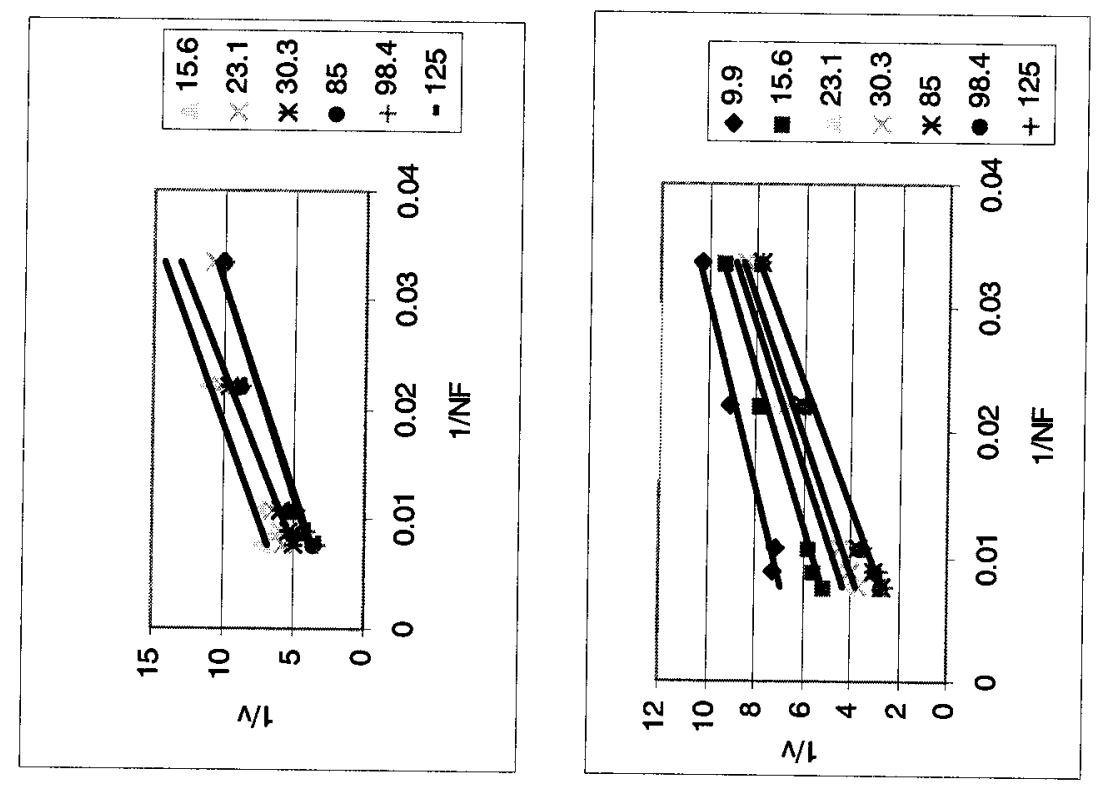

퓨

峦蛋

点菦

方

少

은

总总

营

号

至哭

营

氕

눙

늘

营

总主

릉
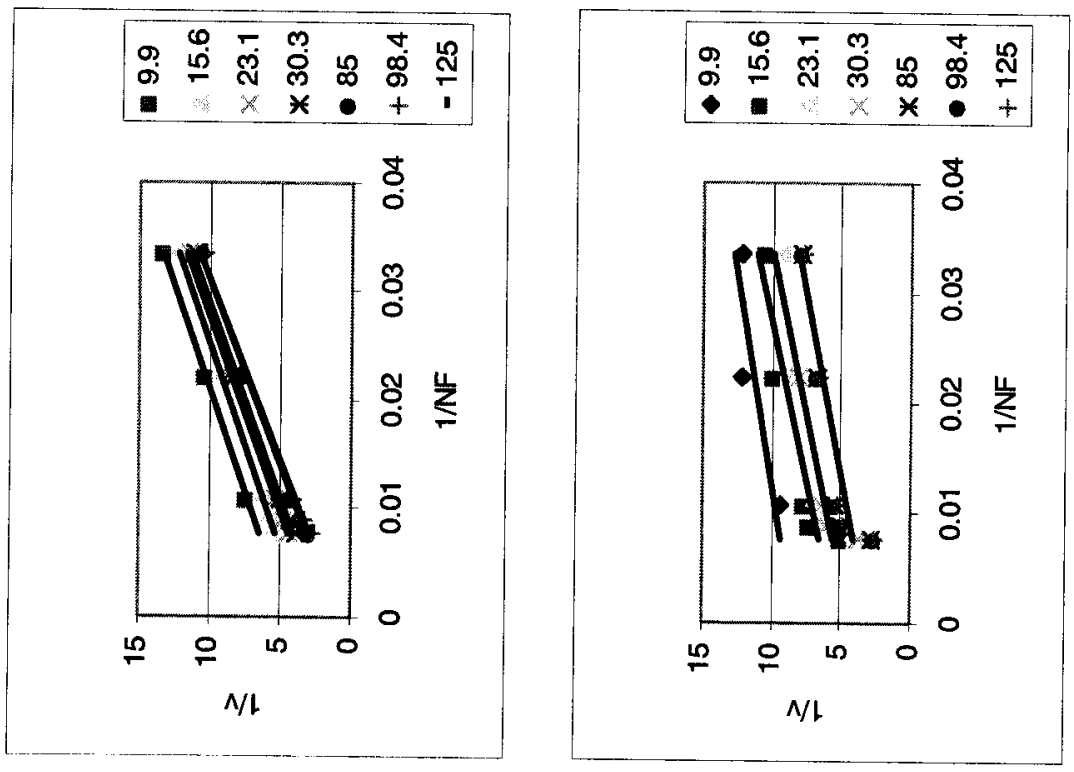

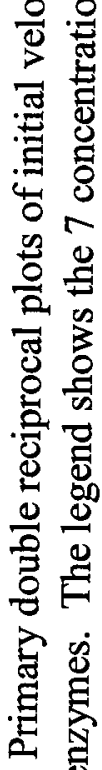

훙

范

造至 

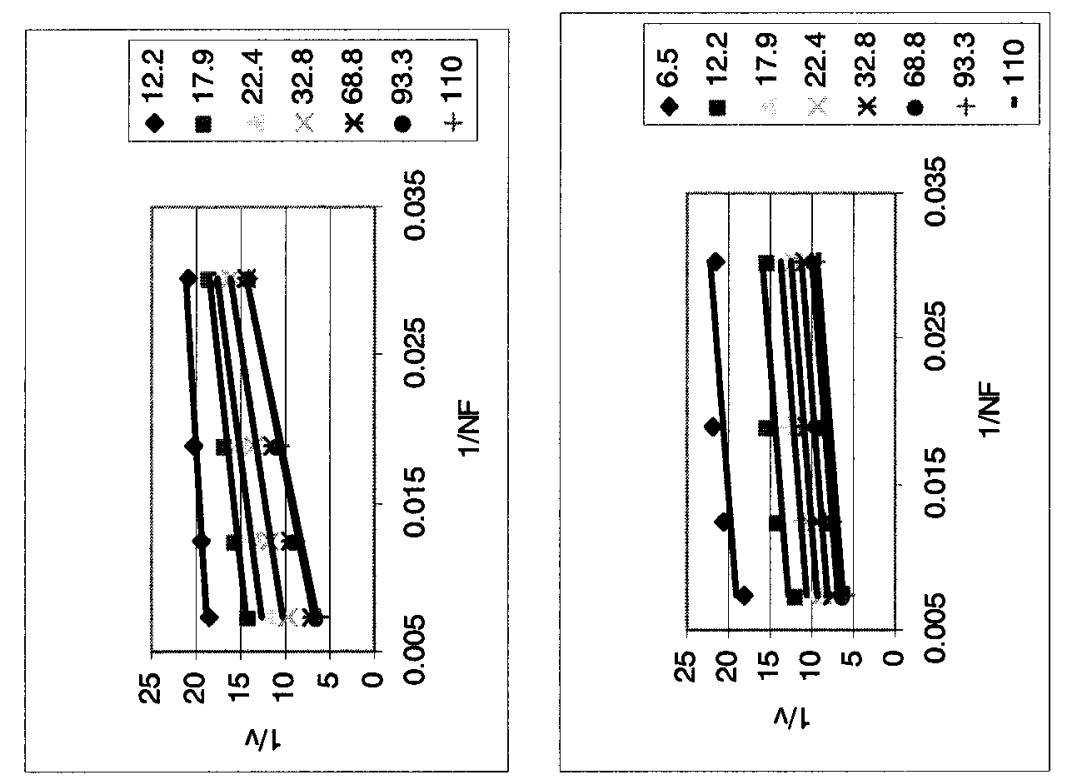

壱

番苍

䒠

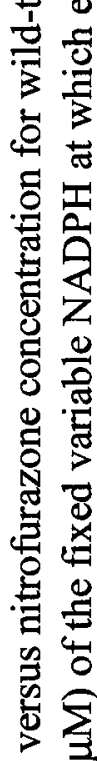

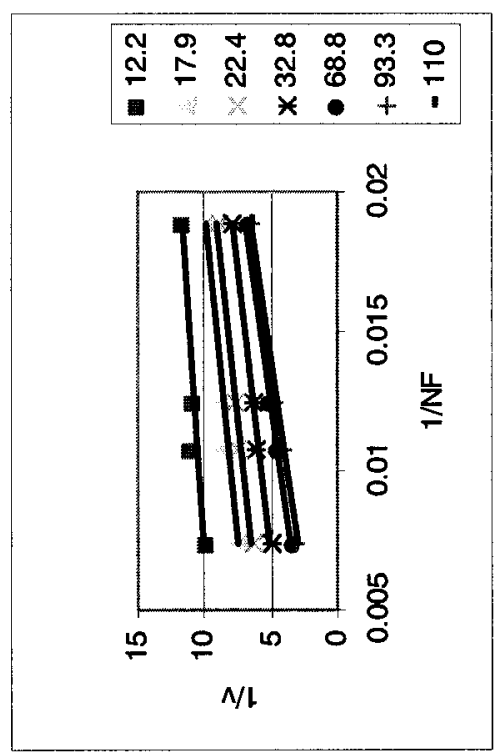

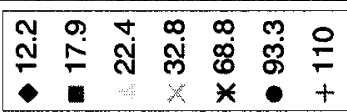

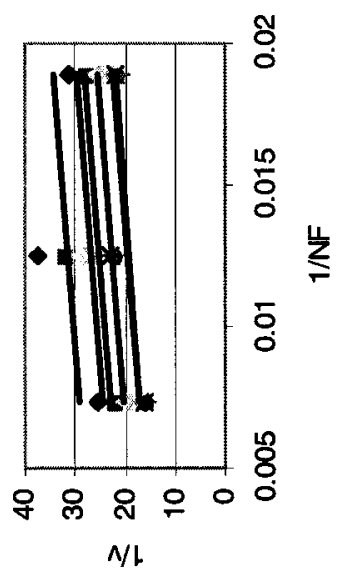

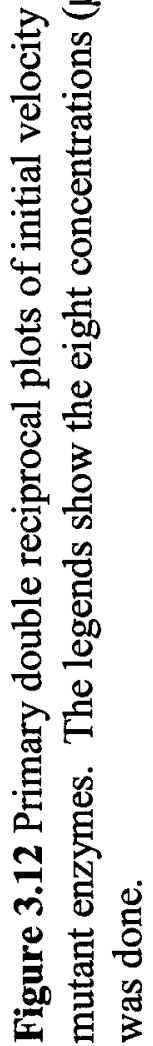




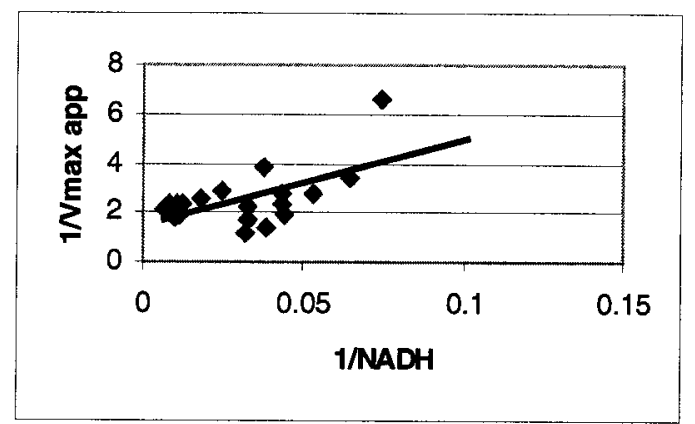

Wild-type

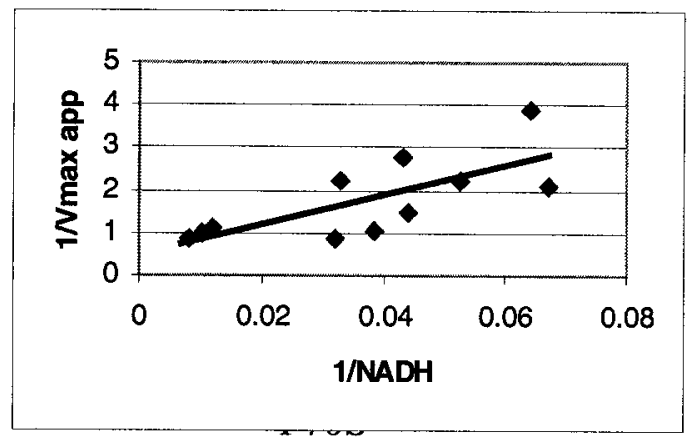

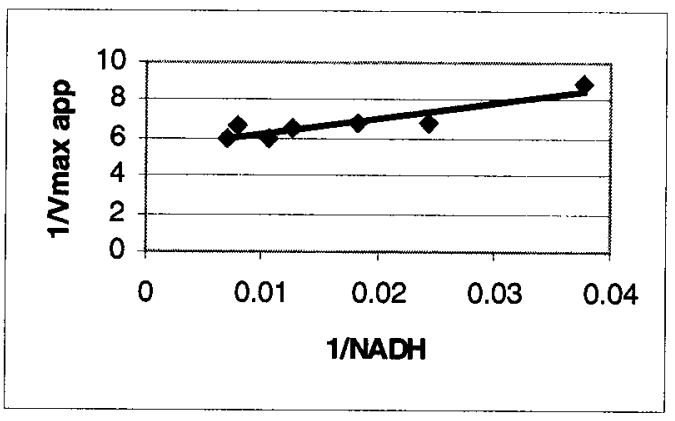

G158S

Figure 3.13 Secondary double reciprocal plots of $V m^{a x}{ }^{\text {app }}$ versus NADH concentration. The data for VAFS were associated with such large errors that a plot was meaningless, and thus was not included. 


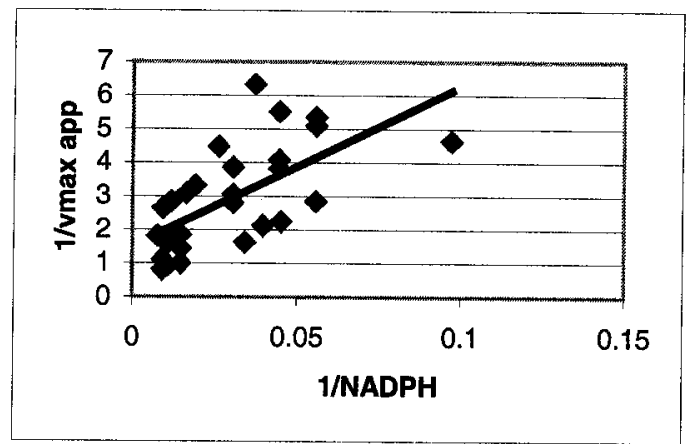

Wild-type

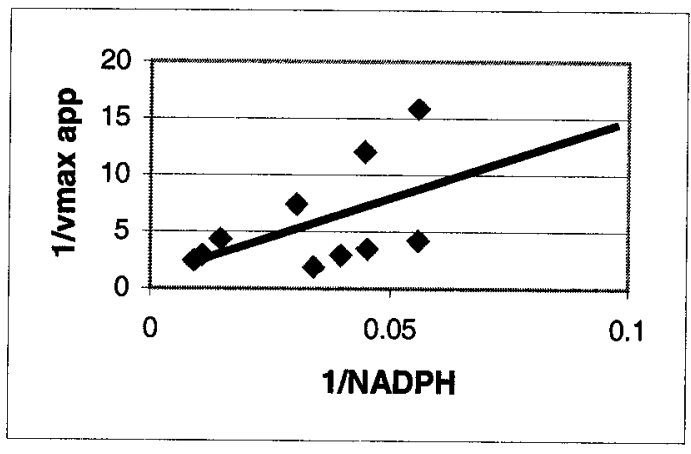

VAFS
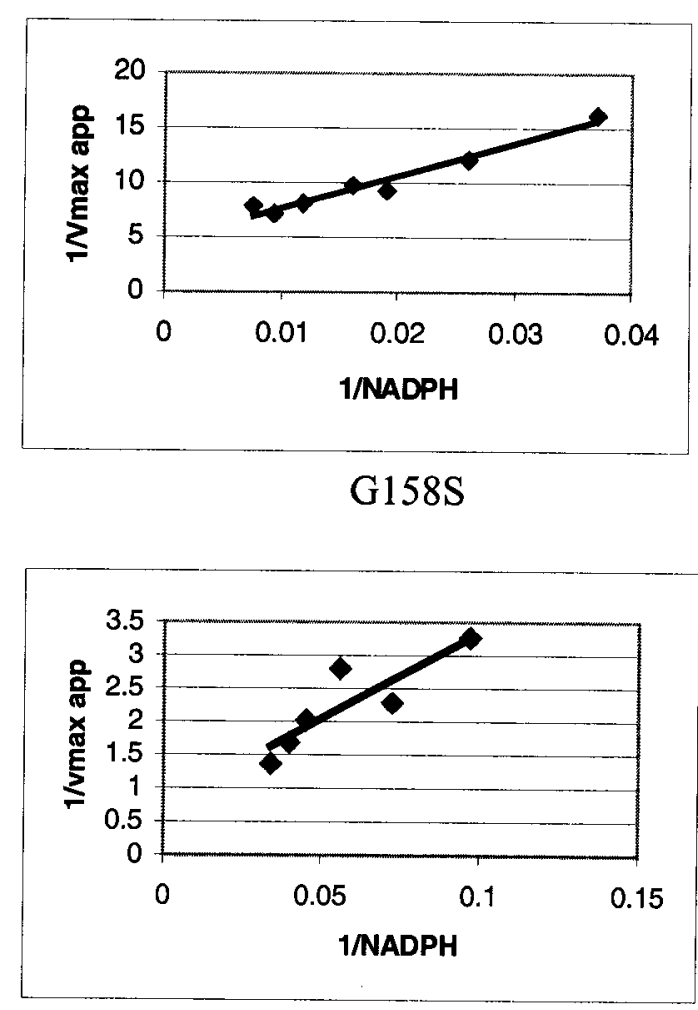

F70S

Figure 3.14 Secondary double reciprocal plots of $\mathrm{Vmax}^{\text {app }}$ against NADPH concentration. 


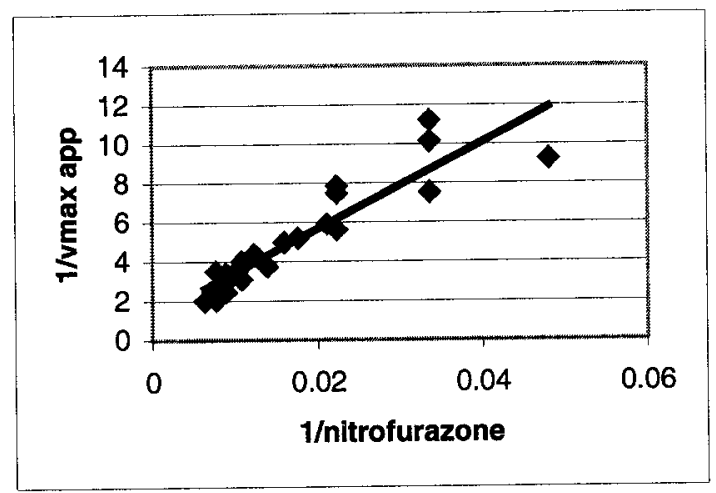

Wild-type

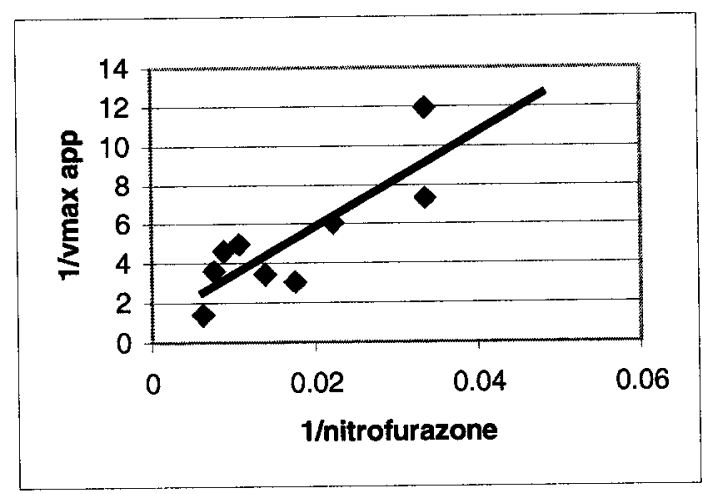

VAFS

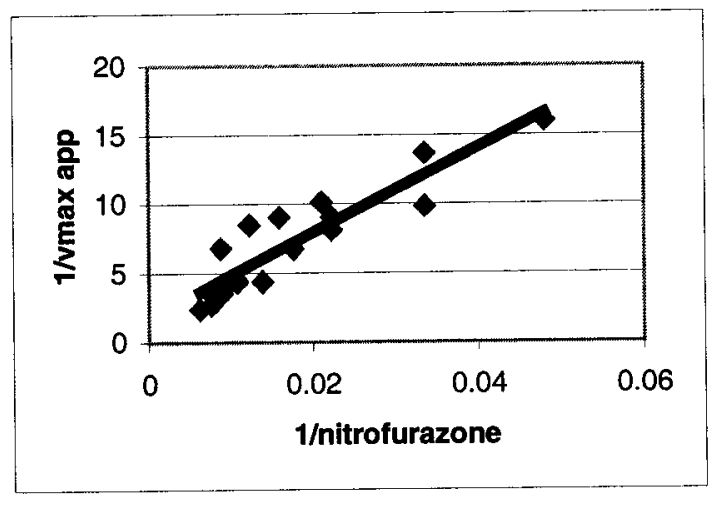

\section{G158S}

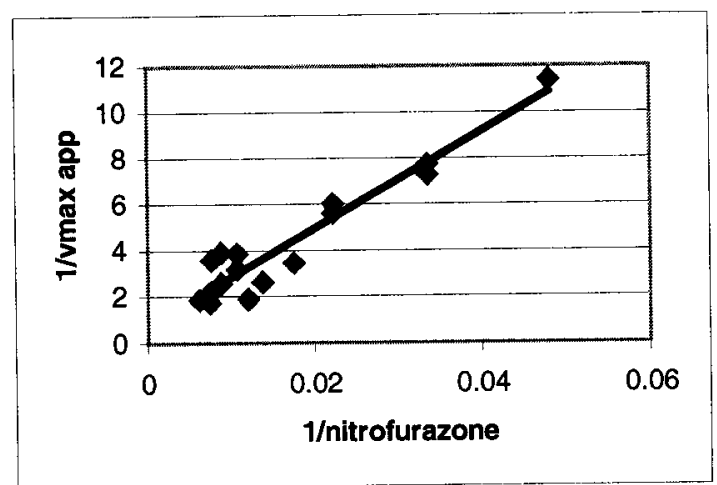

F70S

Figure 3.15 Secondary double reciprocal plots of $\operatorname{Vmax}^{\mathrm{app}}$ versus nitrofurazone concentration with $\mathrm{NADH}$ as the cofactor. 


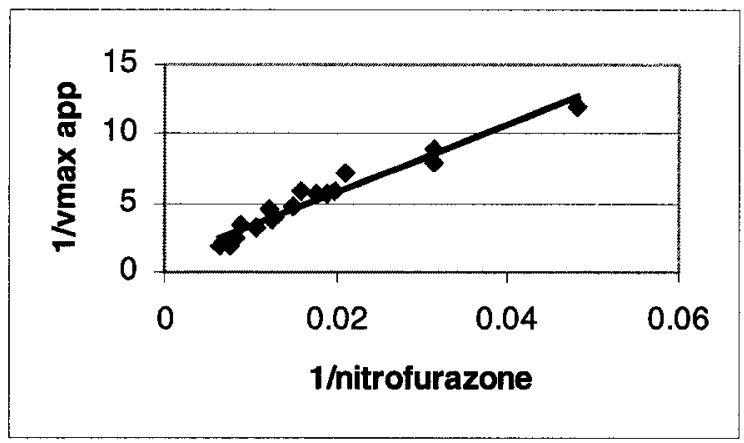

Wild-type

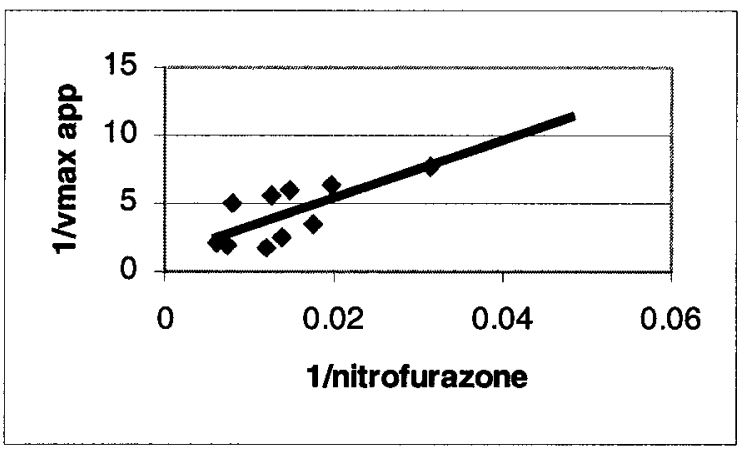

VAFS

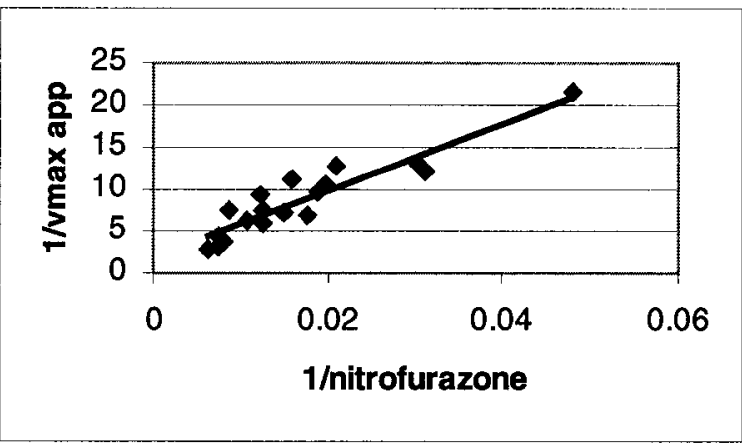

G158S

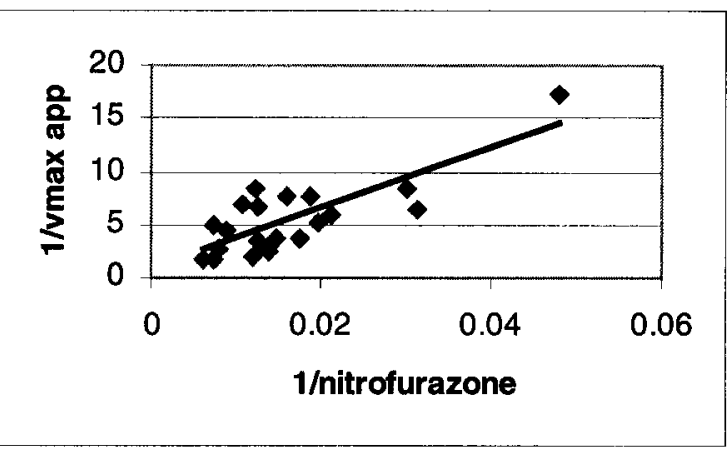

F70S

Figure 3.16 Secondary double reciprocal plots of $\operatorname{Vmax}^{\text {app }}$ versus nitrofurazone concentration with NADPH as the cofactor. 


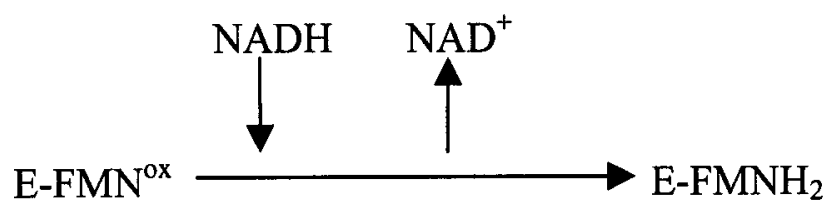

(1)

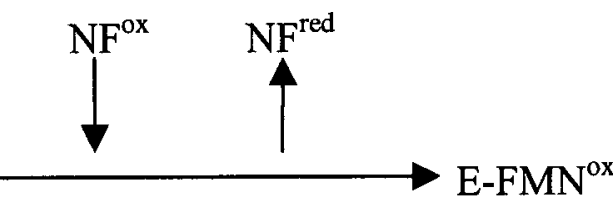

(2)

Figure 3.17 Schematic diagram of a ping-pong reaction mechanism (adapted from Zenno et al., 1996b). $\mathrm{E}=$ Native NfsB. E-FMN and E-FMNH ${ }_{2}$ represent the complexes between $\mathrm{NfsB}$ and $\mathrm{FMN}^{\mathrm{os}}$ and $\mathrm{FMN}^{\mathrm{red}}$, respectively. Between each form of enzyme, (quaternary) transitory complexes are formed which cannot participate in reactions.

(1) Either 2- or 4-electron transfer to bound FMN. Zenno et al. (1996b) suggest that it is a 2-electron transfer step.

(2) Transfer of the electrons to the substrate nitrofurazone. This step either yields the intermediate nitroso derivative, or the fully reduced amino end product. According to a hexa uni ping-pong terreactant reaction mechanism, the substrate (NF) would remain bound here until fully reduced by four electrons. 
for the cofactor itself, $\mathrm{Km}_{(\mathrm{NAD}(\mathrm{P}) \mathrm{H})}$, which approximates the affinity of the enzyme for the coenzyme. Here, the $\mathrm{Km}_{\mathrm{NADH}}$ of $\mathrm{NfsB}, 25.1 \mu \mathrm{M}$, is slightly lower than the $\mathrm{Km}_{\mathrm{NADPH}}$, which is $31.8 \mu \mathrm{M}$. The second $\mathrm{Km}$ value is for nitrofurazone in order to approximate the affinity of $\mathrm{NfsB}$ for the electron acceptor. In this study the $\mathrm{Km}$ value for nitrofurazone was calculated with each cofactor to yield two values, $\mathrm{Km}_{\mathrm{NF}(\mathrm{NADPH})}$ and $\mathrm{Km}_{\mathrm{NF}(\mathrm{NADH}) \text {. }}$ The $\mathrm{Km}$ values for nitrofurazone were larger than for the cofactors, and the $\mathrm{Km}$ of nitrofurazone with NADPH $(275 \mu \mathrm{M})$ is greater than that with NADH $(174 \mu \mathrm{M})$.

Two secondary double reciprocal plots were made, one for the cofactor (Figures $3.13 \& 3.14$ ) and one for nitrofurazone (Figures $3.15 \& 3.16$ ), and two Vmax values were obtained for each cofactor. The turnover number, or $\mathrm{k}_{\mathrm{cat}}$, was calculated from an average of these two Vmax values. The turnover number of nitrofurazone was slightly higher with NADPH as the coenzyme, though close to $100 \mathrm{sec}^{-1}$ with each cofactor (Table 3.4).

Catalytic specificity, calculated as $\mathrm{k}_{\mathrm{cat}} / \mathrm{Km}\left(\mu \mathrm{M}^{-1} \mathrm{~min}^{-1}\right)$, is the most important measure when assessing changes in an enzyme, particularly changes with regards to specific substrates (Copeland, 2000; Plesner, 1986). The catalytic specificity of NfsB for nitrofurazone with each cofactor, $\mathrm{k}_{\mathrm{cat}} / \mathrm{Km}_{\mathrm{NF}(\mathrm{NAD}(\mathrm{P}) \mathrm{H})}$ (Figure 3.18a), and with each cofactor, $\mathrm{k}_{\mathrm{cat}} / \mathrm{Km}_{\mathrm{NAD}(\mathrm{P}) \mathrm{H}}$ (Figure 3.18b), was also calculated independently. A comparison of catalytic specificities for the cofactors and nitrofurazone was accomplished by determining the absolute ratio of NADH:NADPH $\mathrm{k}_{\mathrm{cat}} / \mathrm{Km}$ values (Figure 3.18c). Thus, an absolute ratio of greater than one indicates a preference for NADH. Again, both sides of the reaction must be considered, and hence a comparison of cofactor specificity (cofactors on Figure 3.18) and nitrofurazone specificity calculated 


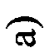

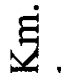
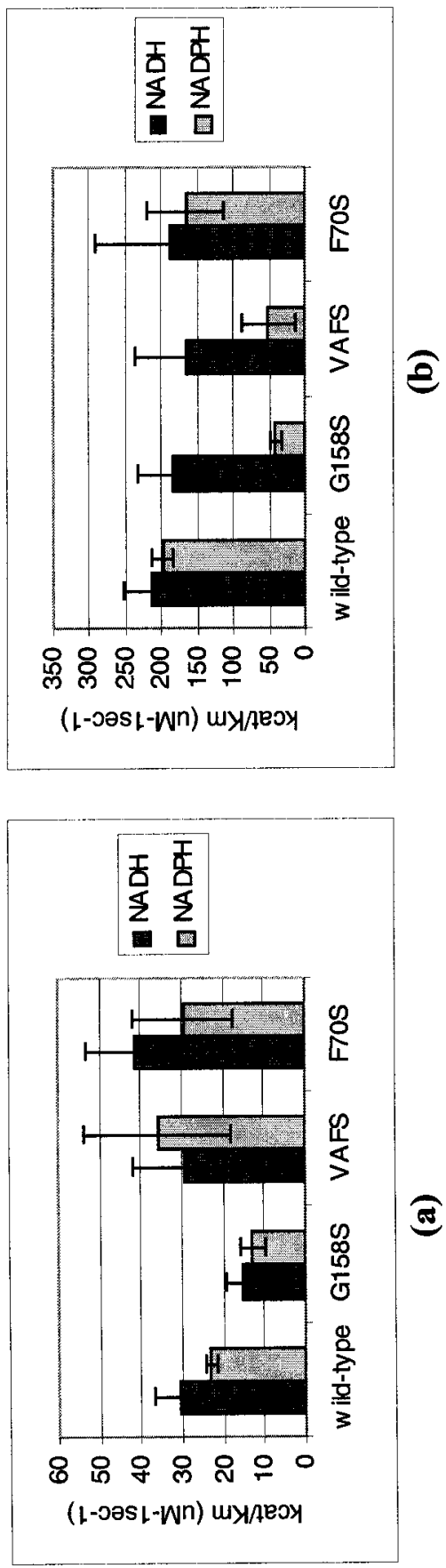

$\widehat{\varrho}$

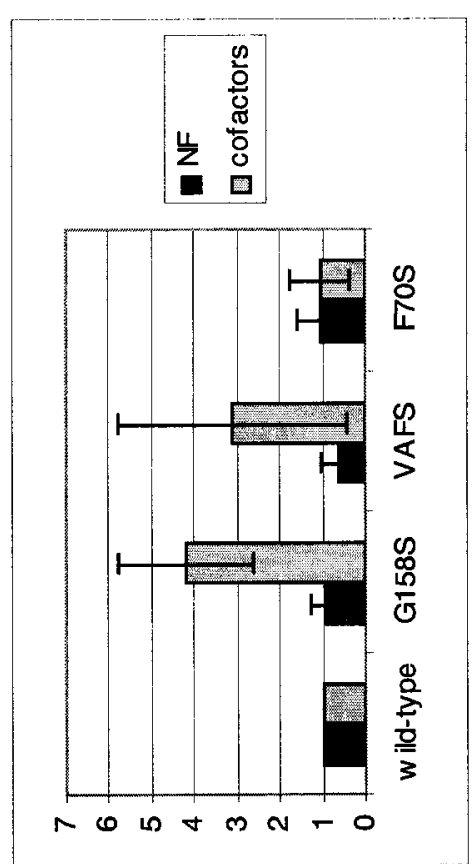

?

是曹

的政

乙解要

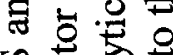

红

䨪

ज टै है

议

记要孚

论

주ㅇㅝㅓㅇ

鞄

कै 造

青宓出

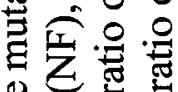

记

总

卷焉造

艺

实范

客零

亭这乐

结

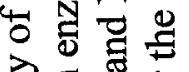

突 产

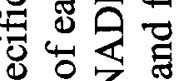

के

它部

要窎

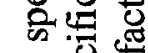

$\infty$.

ते 专

- 范

bo 至焉 
with each cofactor (NF on Figure 3.18) is necessary. The NF and cofactor absolute ratios for wild-type $\mathrm{NfsB}$ of 1.3 and 1.1, respectively, indicate a preference for NADH (Figure 3.18c).

\subsubsection{PCR mutagenesis}

The stretch of amino acids from G153 to L159 in NfsB was selected as a mutagenesis target (Figure 3.1b) to determine the relevance, if any, of these residues on $\mathrm{NAD}(\mathrm{P}) \mathrm{H}$ usage. Rather than introduce single mutations by means of numerous individual site-directed mutagenesis reactions, a two-step PCR mutagenesis approach, using primers degenerate at 7 positions, allowed numerous distinct missense mutations to be obtained simultaneously within the target region (Figure 3.3).

In initial attempts to create mutations within the $n f s B$ sequence encoding Gly-153 to Leu-159, direct insertion of the amplified mutated DNA fragment into the expression vector pIT was attempted by using the lower PCR primer, IMPBL, during the second round of PCR. This approach was arduous and ineffective as the low copy number of pIT was not amenable to manipulation and the SapI digestion was often incomplete, which usually led to aberrant clones (i.e. containing truncated or mutated DNA, or inserts of unknown origin). These problems were circumvented by: (1) use of a different primer, ILH, that is within the intein coding region and contains a 5' HindIII site, and (2) insertion of the amplified fragment into $\mathrm{pCR}^{\circledR} 2.1$ with subsequent subcloning into $\mathrm{pIT}$. The direct insertion of PCR amplified fragments into the high copy number vector $\mathrm{pCR}^{\circledR} 2.1$ (as explained in Section 3.2.7) facilitated sequencing and subcloning, especially since blue/white screening of inserts on medium containing Xgal was possible. Indeed, 


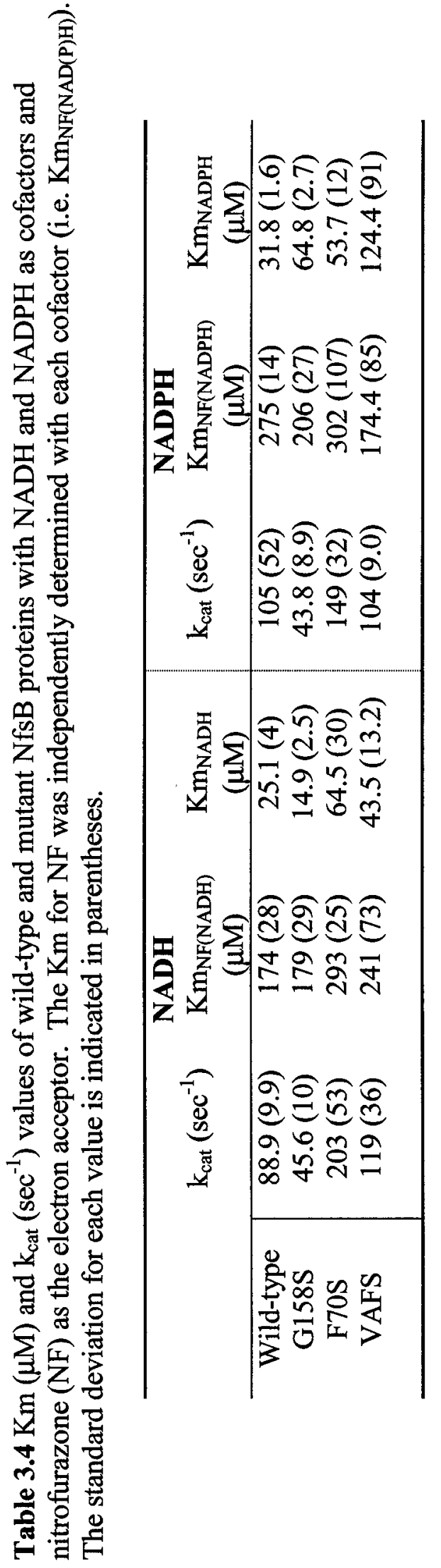


$104 / 167(62 \%)$ of the white $\mathrm{pCR}^{\circledR} 2.1$ transformants contained the correct insert compared to only $40 / 153(26 \%)$ of plasmids with inserts in the original pIT cloning approach. Moreover, where the pIT clones were usually aberrant, the $\mathrm{pCR}^{\circledR} 2.1$ clones usually contained the entire and correct insert.

The target region of the 104 transformants with the correct insert was sequenced. Table 3.5 lists the mutations of the screened transformants. In six mutants the sequence was illegible and analysis was not continued. Table 3.6 summarizes the frequency of the mutation number (single, double, etc.) obtained in the target region of $n f_{s} B$ in the 98 transformants for which unambiguous sequence was ascertained. A single mutation within the target region was desired, but with 7 degenerate positions multiple mutations were expected in the majority of cases. The ratio of correct to contaminating base at each target site was 0.625 , which was predicted to yield $15.6 \%$ of PCR generated oligonucleotides with a single mutation (see Section 3.2.7). In actual fact, 30, almost onethird, of the correctly cloned fragments had a single mutation, of which 16 (out of a possible 21) were unique. Mutation frequency at the target sites was not equal with half (15) of all single mutations obtained at two positions: 461 (7 mutants) and 470 (8 mutants).

Figure $3.1 \mathrm{~b}$ and Table 3.5 illustrate all of the potential missense mutations and indicate the 16 (of 21 possible) unique missense mutations that were obtained. Mutants that contained inserts with a single mutation were further characterized by sequencing the entire $n f_{S} B$ gene. With the one exception of mutant \#96, no additional mutations were found within the $n f s B$ coding region. The extra mutation in mutant $\# 96$ was $\mathrm{T}^{209} \rightarrow \mathrm{C}$, 
Table 3.5 Mutations introduced by targeted PCR mutagenesis. The target region includes residues Gly-153 to Leu-159 in NfsB. The targeted nucleotides are indicated above their encoded residues. Figure $3.1 \mathrm{~b}$ shows all of the possible mutations in the target region.

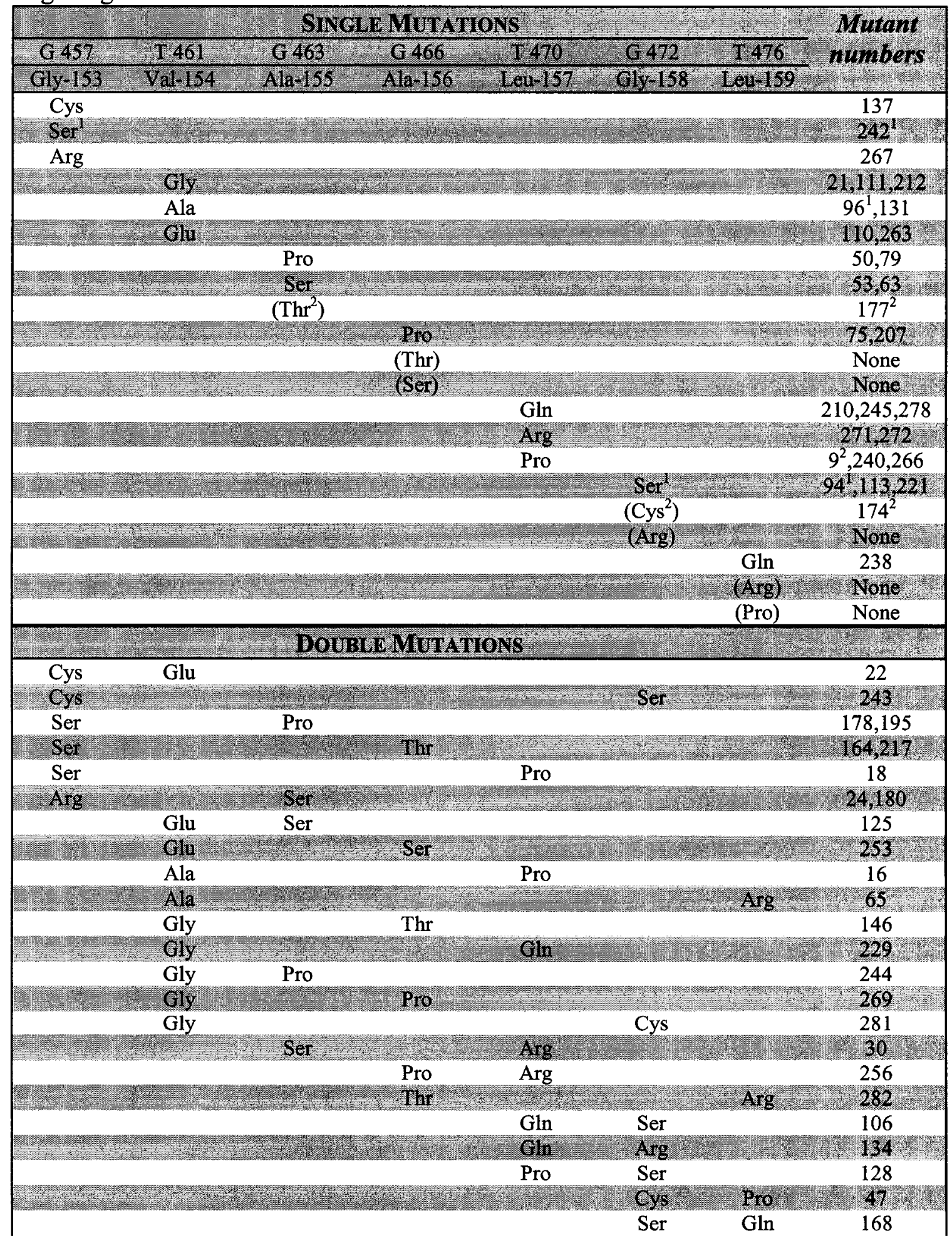


Table 3.5 cont'd.

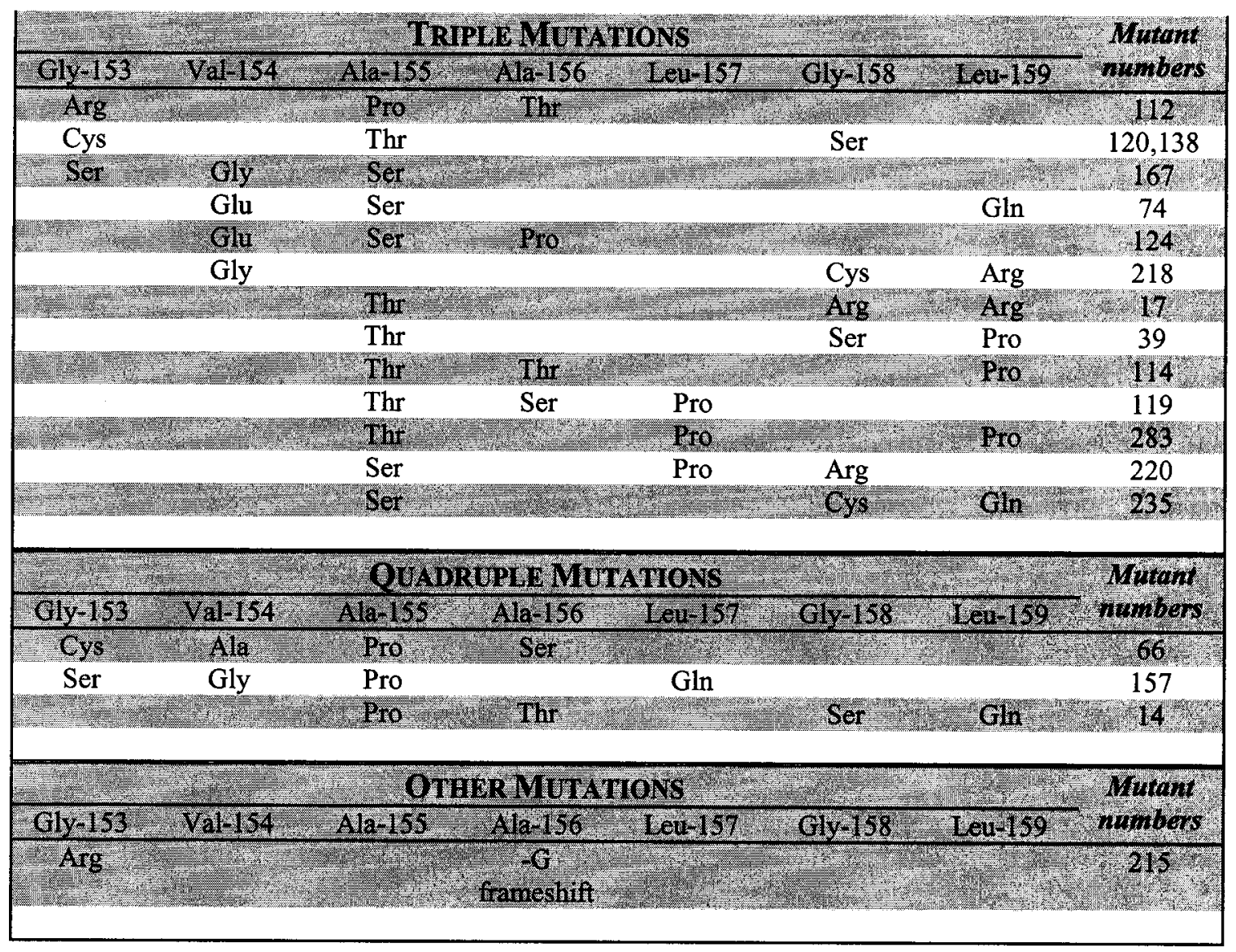

Note: Parentheses indicate that the mutation could not be tested, as either the mutation was not obtained, or the protein could not be expressed.

${ }^{1}$ Mutants had some NR activity and the proteins were analyzed further.

${ }^{2}$ The protein was not induced so analysis was not possible. In the cases of \#174 and \#177 where the mutation was not otherwise obtained these mutations could not be studied. 
Table 3.6 Predicted frequency of mutation number in the target region of $\mathrm{NfsB}$, and the actual frequency in 98 mutants.

\begin{tabular}{ccc}
\hline No. of mutations & Frequency (\%) & Predicted Frequency (\%) \\
\hline 0 (wild-type) & $24.5(24)$ & 3.7 \\
1 & $30.6(30)$ & 15.6 \\
2 & $26.5(26)$ & 28.2 \\
3 & $14.3(14)$ & 28.2 \\
4 & $3.1(3)$ & 16.9 \\
$?$ & $1(1)$ & -- \\
other & -- & 7.4 \\
\hline
\end{tabular}

Note: Numbers in parentheses are the actual number of mutants 
which translates to Phe-70 $\rightarrow$ Ser in NfsB. All products with unique single mutations, plus that of mutant \#96, were subcloned into pIT. As well, 4 double mutation and 3 triple mutation inserts were subcloned (refer to Table 3.5). The two mutations in isolate \#96 were subsequently separated. The $\mathrm{T}^{461} \rightarrow \mathrm{C}(\mathrm{Val}-154 \rightarrow \mathrm{Ala})$ mutation was easily subcloned, but the $\mathrm{T}^{209} \rightarrow \mathrm{C}$ (Phe-70 $\rightarrow$ Ser) mutation was more difficult. A construct was finally obtained by reversing the gene's orientation during the intermediate cloning step (insertion into a pUC vector) so that it would not be expressed during manipulations (see Section 3.2.8.2).

\subsubsection{Purification and characterization of mutants}

\subsubsection{NR activity}

Mutants were selected for kinetic analysis by screening for enzyme activity. Two of the mutants, \#174 and \#177, did not express the triple fusion protein and further analysis were not possible. The nitroreductase (NR) activity of the remaining 14 mutant proteins was measured both in a crude extract and after purification. In cell extracts, most of the mutants had nitroreductase activity similar to that of the host strain GJ1158 alone $(\sim 18 \mu \mathrm{mol} / \mathrm{min} / \mathrm{mg})$; the only mutants with NR activity significantly greater, that is with a difference of at least two standard deviations, than GJ1158 were \#94 (G158S), \#96 (V154A, F70S) and \#242 (G153S) (Table 3.2). Herein each mutant is referred to according to its amino acid mutation, shown in parentheses above, except for isolate \#96, which has two mutations and is designated VAFS. Each of these mutants also displayed activity when assayed with NADPH as a cofactor. In cell extracts, the mutants generally exhibited less NR activity than vector-encoded wild-type NfsB. The exception is VAFS, 
which often demonstrated greater overall NR activity than wild-type $\mathrm{NfsB}$, although there were large fluctuations in NR activity between different preparations of VAFS. This fluctuation in NR activity for VAFS was also apparent in purified preparations of the enzyme and is probably due to variations in the stability of the enzyme as a consequence of the two mutations.

NfsB from all of the mutants, even those without discernable cell extract activity, was purified and the NR specific activity was measured. Sufficient enzyme was obtained to determine the specific activity of the 14 mutant enzymes even though expression levels of the mutants were usually lower than for the wild-type protein, and aggregation was often a hindrance during purification (data not shown). Mutants with low specific activity in the cellular extract had correspondingly low activity after purification. The mutant with the lowest assayable activity, G153S, had such low overall activity that kinetic analysis was not possible. The Val-154 $\rightarrow$ Ala substitution subcloned from VAFS resulted in the elimination of NR activity, while F70S retained full NR activity.

\subsubsection{Structural integrity}

After the nucleotide sequences of the mutant genes were confirmed, and the molecular weight of the proteins was established by SDS-PAGE, the structural integrity of the enzymes was examined using fluorescence spectroscopy. Fluorescence spectra of $1 \mu \mathrm{M}$ G158S, VAFS, V154A, F70S, G153S and $0.5 \mu \mathrm{M}$ of the null mutant A156P were obtained for both tryptophan and FMN, and compared to the wild-type spectrum (Section 3.3.2.2). Spectra of the other mutants could not be obtained, as concentrations of the enzyme preparations were too low. 
Changes in the Trp fluorescence spectra of the NfsB mutant proteins contribute information on the overall structural integrity of $\mathrm{NfsB}$, but could also indicate perturbation near the putative substrate binding site. The three tryptophan residues (W46, W94, W138) in NfsB are within approximately $10 \AA$ of one another W138 is in the FMN binding pocket of NfsB, and W46 is a highly conserved residue among the NfsB homologues that may be involved in substrate binding (see Chapter 4, Section 4.4.2.3). NfsB proteins that exhibit considerable NR activity, such as the wild-type, VAFS and F70S, had an emission peak at $345 \mathrm{~nm}$. A peak of significantly less intensity was evident at $375 \mathrm{~nm}$ for the mutants that exhibit less NR activity (Table 3.3). This shift to a longer wavelength suggests that at least one of the Trp residues is more exposed to the solvent in the mutant enzyme as compared to the wild-type; completely buried tryptophan residues generally exhibit emission maxima near $320 \mathrm{~nm}$ (Creighton, 1993). The $375 \mathrm{~nm}$ peak is evident only in the target region mutants, but VAFS also has a mutation within this region (V154A) and does not have a peak at this wavelength. Similarly, the FMN spectrum of VAFS resembles that of F70S, rather than V154A and the other mutants with less NR activity (discussed below). Thus, spectral variations appear to reflect differences in NR activity, suggesting that the diminished NR activity of the mutant enzymes is due at least in part to structural changes.

The UV absorbance at $280 \mathrm{~nm}$ was obtained for $0.1 \mu \mathrm{M}$ of the same protein preparations used for the fluorescence spectra (Table 3.3). F70S and VAFS had absorbance values similar to the wild-type protein, about 0.2 , while the other mutants had absorbance values greater than 0.35 . Indeed, the least active mutants G153S, V154A and 
A156P had the highest absorbance values $(0.5-0.6)$. This correlation with the fluorescence spectra peaks emitted after Trp excitation may also indicate that the aromatic residues are more exposed to the solvent in the less active mutants.

Emission scans of the mutants after excitation of the FMN molecule at $464 \mathrm{~nm}$ exhibited some differences from the wild-type scan (Figure 3.8b). Although an equal concentration of each enzyme was used ( $1 \mu \mathrm{M}$ except for $0.5 \mu \mathrm{M}$ A156P), the intensities of some spectra, particularly those of VAFS and G153S, were different, which suggests that the environment of the FMN molecule has changed. Unlike absorbance, fluorescence usually does not follow Beer's law and greater concentrations of enzyme can actually yield less fluorescence due to the inner filter effect (Munro and Noble, 1999), while buried residues often produce peaks of greater intensity than exposed residues (Copeland, 2000).

The shapes of the spectra are also indicative of structural alterations around residues that influence FMN binding. As described in Section 3.3.2.2, peak I ( $525 \mathrm{~nm})$ is more defined and is of greater intensity than peak II $(550 \mathrm{~nm})$ in wild-type NfsB. Likewise, ratios of peak I:peak II in the mutant enzymes with NR activity are greater than one (Table 3.3). Peak II is practically absent for F70S and VAFS, such that their spectra resemble the single peak of free FMN (Figure $3.8 \mathrm{~b}$ ). In addition, there is a slight blue shift, to 520nm, of peak I in the F70S and VAFS mutants. In V154A peak I underwent a slight red shift, to $\sim 528 \mathrm{~nm}$. Peak II is most pronounced for V154A and A156P, the mutants with no detectable NR activity (peak I:peak II ratios of 1.1 and 0.9 respectively). Furthermore, peak II undergoes a slight red shift, to $555 \mathrm{~nm}$, in these mutants. The 
mutants with intermediate NR activity, G153S and G158S, exhibit an intermediate ratio (1.2) of peakI:peakII.

The spectral perturbations observed for the A156P, V154A and G153S mutants are probably due to disruption of the $\alpha \mathrm{G}$ helix (Figure 3.19). Simulation of the mutated residues in the three-dimensional structure of NfsB with the program SWISS-PDBviewer (Guex and Peitsch, 1997) demonstrated that many new bonds are probably formed in these mutant proteins, whereas new bonds are not predicted to form with the F70S mutation. It is unclear why the spectra of VAFS resemble F70S despite the V154A mutation.

\subsubsection{Enzyme kinetics}

The reaction mechanism and kinetic parameters of the mutant enzymes VAFS, F70S and G158S were analyzed and compared to wild-type NfsB. Kinetic analysis of G153S was not possible as this mutant enzyme has too little activity to obtain a sufficient range of initial velocities. The difference in initial velocities was negligible for the range of substrate concentrations necessarily used here due to equipment limitations (refer to Section 3.3.2.3). Kinetic analysis of the bisubstrate NfsB enzyme involves the determination of at least four reaction curves (refer to Section 3.3.2.3). If much higher concentrations of nitrofurazone could be used, then it would be possible to obtain a few enzyme reaction curves with significantly variable initial velocities such that the actual Vmax and $\mathrm{Km}$ values of G153S could be determined from the primary plots. 
Figure 3.19 Nucleotide sequence of the $n f s B$ gene with the translated sequence below it. Mutagenesis target region is shown in the box. Rectangles above the sequence indicate the alpha helices $(\alpha)$ and beta sheets $(\beta)$ of the secondary structure derived from the 3D structure of NfsB (Parkinson et al., 2000). Restriction endonuclease sites are underlined in the nucleotide sequence and identified above the sequence. The primers used for amplification and cloning of the mutated fragment are shown as arrows above the nucleotide sequence. 


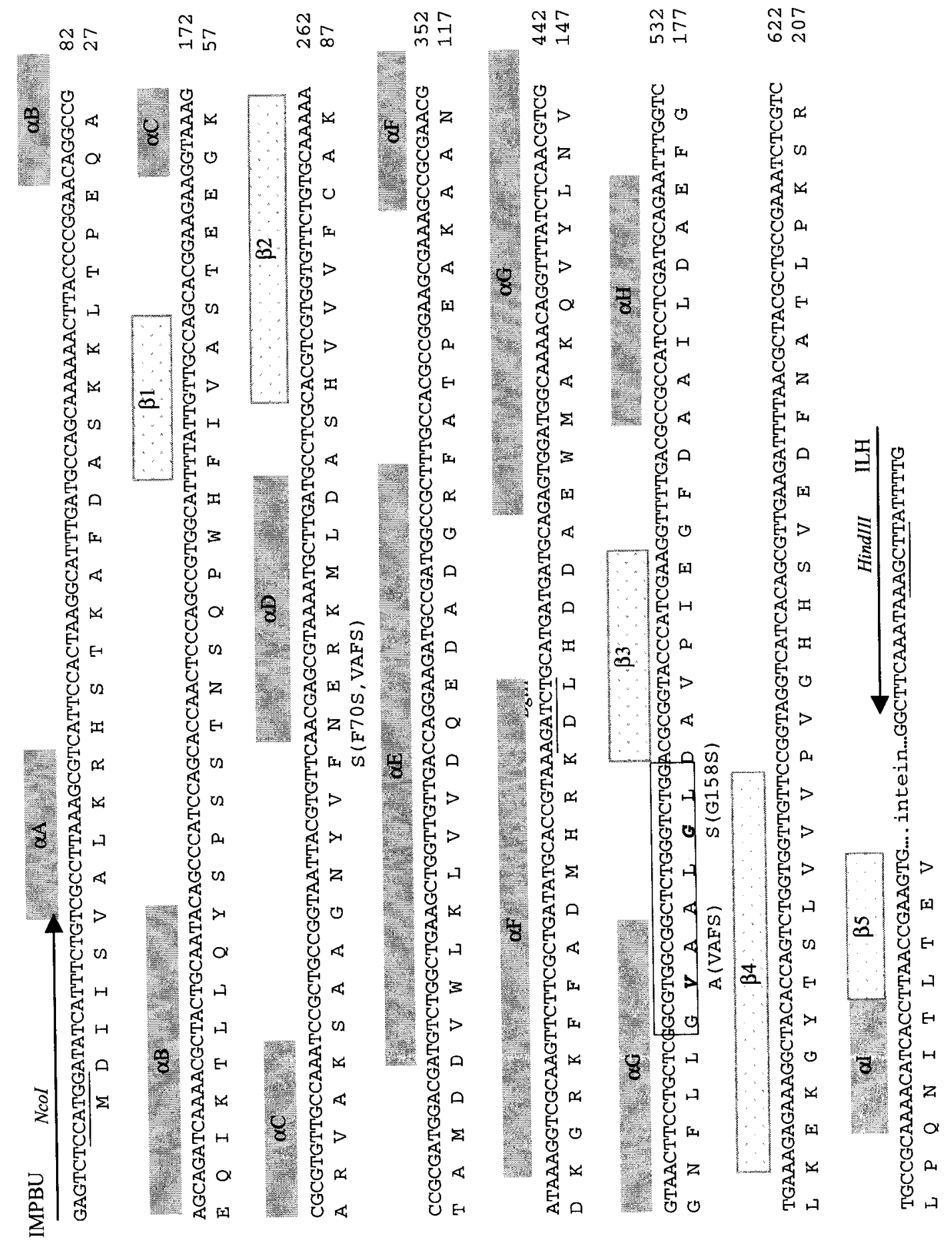




\subsection{Mechanism}

At least three primary double reciprocal plots from independently determined data were obtained for the mutant enzymes. Figures 3.9-3.12 illustrate the representative plots. In most cases a ping-pong $\mathrm{Bi}-\mathrm{Bi}$ mechanism is implicated for the mutant enzymes. Parallel lines were obtained for the primary double reciprocal plots with NADH (Figure 9b-d), and G158S and F70S exhibit parallel lines with NADPH (Figure 10b,d). One possible exception is revealed by the slight convergence of lines for VAFS with NADPH (Figure 10b). However, large errors associated with VAFS preclude any conclusions regarding its reaction mechanism.

Another possible deviation from a ping-pong $\mathrm{Bi}-\mathrm{Bi}$ mechanism is detected in the plots of G158S (Figures 3.9b \& 3.10b). At increased concentrations of nitrofurazone there is an increase in the slope values derived from the $1 / \mathrm{Vi}$ versus $1 /[\mathrm{NADPH}]$ plot. This trend does not conform to a recognized pattern of reaction mechanism (e.g. ordered or random) (Segel, 1975). The lines may essentially be parallel as the deviation is slight and within the limits of error, however, it could also be an indication of substrate inhibition. A slight increase in slope values is also apparent for the $1 / \mathrm{Vi}$ versus $1 /[\mathrm{NADH}]$ plot, but if substrate inhibition is present for this cofactor it is less pronounced than for NADPH.

\subsection{Substrate inhibition}

Competitive substrate inhibition is distinguished by a linear replot of slopes from the primary double reciprocal plot against the inhibitory substrate (Segel, 1975). Here, the slopes of the $1 / \mathrm{Vi}$ vs $1 /[\mathrm{NADPH}]$ plot for G158S (Figure 3.10b) versus nitrofurazone 
concentration yield a line with a slope of 0.68 (Figure 3.20). A similar replot for wild-type gives a lesser slope of 0.29 and F70S yields no positive slope at all. The large sampling fluctuation associated with VAFS prevents a replot for this mutant. This observed linearity may indicate that G158S experiences substrate inhibition by nitrofurazone. If so, then the inhibition constant $(\mathrm{Ki})$ of nitrofurazone can be extrapolated from Figure 3.19 to give approximately $123 \mu \mathrm{M}$ (Segel, 1975). Likewise, the $\mathrm{Ki}$ of nitrofurazone for the wild-type enzyme would be $194 \mu \mathrm{M}$. Table 3.7 lists the possible Ki values derived from the primary $\mathrm{NAD}(\mathrm{P}) \mathrm{H}$ double reciprocal plots. Of course, experimental error or an inadequate range of substrate concentrations may account for these linear slope plots. However, linear plots are not obtained from the $1 / \mathrm{Vi}$ vs. 1/nitrofurazone graphs, which indicates that NADH and NADPH are probably not inhibitory. It should be noted that if the $\mathrm{Ki}$ for a substrate were much greater than its $\mathrm{Km}$ value, then parallel lines would be apparent on the primary double reciprocal plot (Segel, 1975); therefore, it might be that the cofactors are inhibitory but that their Ki values are much greater than their $\mathrm{Km}$ values.

\subsection{Kinetic Parameters}

The kinetic parameters, $\mathrm{Km}$ and $\mathrm{k}_{\mathrm{cat}}$, of the mutant enzymes for the cofactors and nitrofurazone are shown in Table 3.4. As described in Section 3.3.2.3.2, the catalytic specificities for the cofactors and nitrofurazone are compared by an absolute ratio of $\mathrm{NADH}: \mathrm{NADPH} \mathrm{k}_{\mathrm{cat}} / \mathrm{Km}$ values (Figure $3.18 \mathrm{c}$ ). Like wild-type NfsB, all of the enzymes exhibit a preference for NADH: the absolute NADH:NADPH $\mathrm{k}_{\mathrm{cat}} / \mathrm{Km}$ ratios are close to or greater than one for nitrofurazone and the cofactors (Figure 3.18c). 
a.

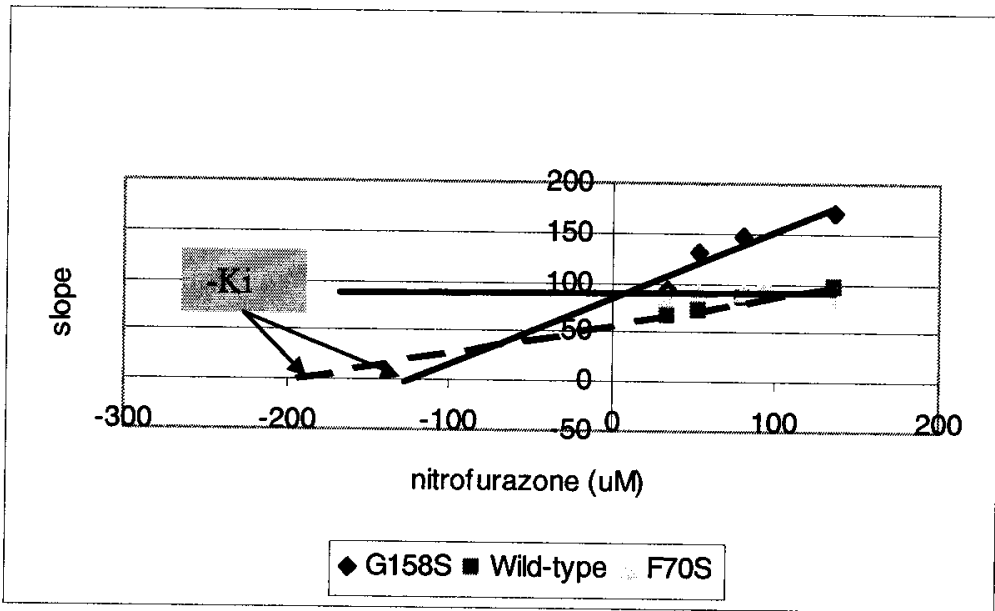

b.

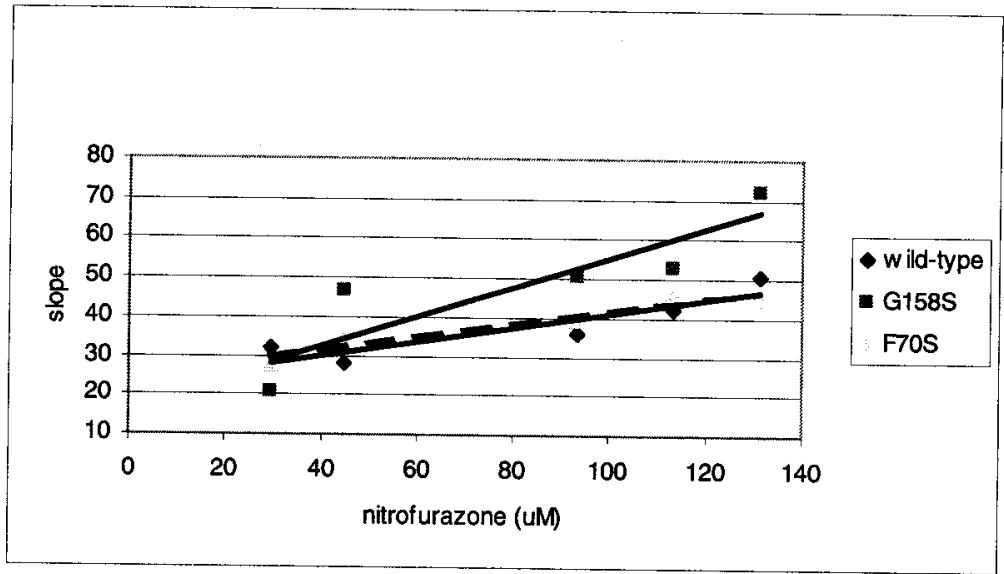

Figure 3.20 Linear replots of slopes at different nitrofurazone concentrations from the double reciprocal plots of $1 / \mathrm{v}$ vs (a) $1 / \mathrm{NADPH}$ and (b) $1 / \mathrm{NADH}$. X-intercepts correspond to $-\mathrm{Ki}$ value if substrate inhibition is present. 
Table 3.7 Ki values $(\mu \mathrm{M})$ for nitrofurazone with each cofactor in wild-type NfsB and two of the mutant enzymes. Refer to Figure 3.20 for the plots from which these values were derived, and Section 3.3.4.1.3.2 in the text for details.

\begin{tabular}{l|cc} 
Protein & \multicolumn{2}{|c}{ Nitrofurazone Ki value $(\mu M)$} \\
Wild-type NfsB & 194 & NADH \\
mutant G158S & 123 & 46.4 \\
mutant F70S & N/A & 146
\end{tabular}


The catalytic specificities of the mutant enzymes for each substrate are compared to those of the wild-type enzyme by a relative mutant:wild-type ratio (Figure 3.18d). A relative ratio greater than one indicates that the NADH specificity of the mutant enzyme exceeds that of wild-type NfsB. Little deviation from the wild-type ratios is seen for nitrofurazone in all of the enzymes, which indicates a similar preference for nitrofurazone with each cofactor in each enzyme. However, compared to the wild-type, F70S yielded similar ratios for the cofactors, while G158S and VAFS exhibited significantly greater specificity for NADH. G158S displayed approximately 4.5 times greater specificity for NADH than NADPH, which is about 4.2 times greater than that demonstrated by wild-type NfsB (Figure 3.18d). The catalytic specificity for NADH is essentially similar for all four enzymes (approximately $180 \mu \mathrm{M}^{-1} \mathrm{~s}^{-1}$ ). The change in cofactor specificity for G158S and VAFS is attributable to a lower specificity for NADPH rather than a heightened one for NADH (Figure 3.18a).

For G158S, the decrease in catalytic specificity for NADPH is a consequence of a higher $\mathrm{Km}$ value and lower turnover number than the wild-type enzyme (Table 3.4). A higher $\mathrm{Km}$ value for NADPH is also indicated for VAFS. The apparently unaffected turnover number of VAFS may be attributable to the increased turnover number contributed by the F70S mutation that could mask a decrease imposed by the V154A mutation (Table 3.4). However, it may be an oversimplification to treat each of these mutations as exerting mutually exclusive and independent effects on the double mutant. The unaffected catalytic specificity for NADH and decreased specificity for NADPH in 
VAFS and G158S, which possess mutations within the target region, suggests that this region is involved in the utilization of NADPH.

By comparison to wild-type NfsB, the decrease in the catalytic specificity of G158S for nitrofurazone is primarily due to a reduction in turnover number; there is little change in $\mathrm{Km}$ values. A turnover number can reflect the transition energy of a reaction (Plapp, 1995), and, especially when there is little change in the Km value (Rescigno and Perham, 1994), this smaller $\mathrm{k}_{\text {cat }}$ value may indicate that the transition energy of G158S is greater than that of the wild-type. 


\subsection{Discussion}

\subsubsection{Wild-type characterization}

\subsubsection{Expression and purification of $\mathrm{NfsB}$}

To date, the purification scheme for NfsB developed in this study is the only one that uses an affinity tag; other researchers have used the more traditional biochemical approach involving a succession of chromatography columns. As discussed in section 3.3.1, a number of problems were encountered during the development of an affinity purification scheme for $E$. coli NfsB. Most of the problems were a consequence of the apparent stickiness of NfsB: aggregation during overexpression, particularly at elevated temperatures; aggregation on the affinity column; and loss of purified protein unless stored in glass tubes with at least $40 \%$ glycerol. By manipulation of expression temperature and buffer constituents, these problems were largely overcome. The purified $\mathrm{NfsB}$ protein obtained by this method has the additional advantage of having no affinity tag.

\subsubsection{Structural integrity}

$\mathrm{NfsB}$, a homodimer, is a flavoprotein with a bound FMN molecule in each of its subunits. The tightly bound FMN molecule is at the core of the active site as it accepts electrons from NAD(P)H and uses them to reduce the nitro-substituted substrate. Thus, the sensitivity of fluorescence spectra to disturbances in the solvent polarity near the fluorophore is an invaluable tool to assess the structural integrity of $\mathrm{NfsB}$, especially at the active site. The fluorescence excitation spectrum of $\mathrm{NfsB}$ exhibits a characteristic flavoprotein peak at $464 \mathrm{~nm}$. Excitation of NfsB at this wavelength results in two 
emission peaks, $525 \mathrm{~nm}$ and $550 \mathrm{~nm}$, that are characteristic of flavoproteins (Munro and Noble, 1999).

Excitation of tryptophan residues at $290 \mathrm{~nm}$ yields a peak at $345 \mathrm{~nm}$, which is typical of buried Trp residues (Creighton, 1993). The Trp spectrum provides an indication of the protein's overall structural integrity. Furthermore, as one Trp residue, $\mathrm{W} 138$, is in the FMN binding pocket of $\mathrm{NfsB}$, it may reflect the integrity of the binding site. However, changes in the Trp spectrum can be attributable to any combination of the three $\operatorname{Trp}$ residues.

\subsubsection{Reaction mechanism}

A previous study concluded that $\mathrm{NfsB}$ reduces nitro-containing compounds according to a ping-pong Bi-Bi mechanism (Figure 3.17) (Zenno et al., 1996b). Evidence for a ping-pong $\mathrm{Bi}$-Bi mechanism is usually, as in the study by Zenno and coworkers, based upon parallel double reciprocal plots. Parallel double reciprocal plots are obtained for NfsB with both NADH and NADPH in this study as well; however, such plots are not definitive of a ping-pong mechanism. For example, an ordered $\mathrm{Bi}-\mathrm{Bi}$ mechanism with a 'sticky' first substrate will also yield parallel lines (Segel, 1975). Detailed studies including inhibitor kinetics and isotope exchange experiments on the NfsB homologue of E. cloacae, NEC, indicate that it does conform to a ping-pong mechanism (Koder and Miller, 1998b), but the researchers did not unambiguously identify a Bi-Bi mechanism either.

The kinetic mechanism of $\mathrm{NfsB}$ must also explain the four-electron reduction of nitro-substituted compounds. Figure 3.17 illustrates the release of a nitroso intermediate 
after the initial two-electron transfer. It is still not clear whether this intermediate is released and rebound, or whether the nitro compound is reduced by four electrons without an intermediate being released. Zenno and coworkers (1996b) state that there is evidence (unpublished) for a two electron transfer constituting the first half-reaction of NfsB's mechanism. Koder and Miller (1998b) addressed this question with NEC by attempting to identify nitroreduction products and intermediates of the simple nitroaromatic compound nitrobenzene. The only compound detected was the fourelectron reduced hydroxylaminobenzene; yet the possible two-electron reduced intermediate, nitrosobenzene, reduces so quickly under the same assay conditions that it was impossible to determine whether the intermediate was released and preferentially rebound (as in a ping-pong $\mathrm{Bi}-\mathrm{Bi}$ mechanism), or if it remained bound to the enzyme. There are instances in which a three-substrate (terreactant) system can yield parallel plots (Fromm, 1979; Segel, 1975). It is thus conceivable that NfsB may follow a terreactant mechanism in which two of the substrates (NADH or NADPH) are electron donors (Koder and Miller, 1998b). For example, a hexa uni Bi-Bi reaction mechanism yields parallel plots for all substrates (Fromm, 1979; Segel, 1975).

\subsubsection{Kinetic parameters}

Table 3.4 reveals the kinetic parameters, $\mathrm{k}_{\mathrm{cat}}$ and $\mathrm{Km}$, obtained for the wild-type and mutant proteins. The $\mathrm{Km}_{\mathrm{NADH}}(25.1 \mu \mathrm{M})$ and $\mathrm{Km}_{\mathrm{NF}(\mathrm{NADH})}(174 \mu \mathrm{M})$ determined for $\mathrm{NfsB}$ in this study are very close to the values, $22 \mu \mathrm{M}$ and $153 \mu \mathrm{M}$ respectively, obtained in a prior study by Zenno and coworkers (1996b). The Km $\mathrm{NADPH}_{\mathrm{N}}(31.8 \mu \mathrm{M})$ and $\mathrm{Km} \mathrm{m}_{\mathrm{NF}(\mathrm{NADPH})}(275 \mu \mathrm{M})$ values are greater than those for NADH. Zenno et al. (1996b), 
who conducted experiments only with $\mathrm{NADH}$, found that the $\mathrm{Km}_{\mathrm{NADH}}$ was lower (5.4 $\mu \mathrm{M})$ when FMN was used as an electron acceptor instead of nitrofurazone. Rejecting the possibility of a different reaction mechanism for flavin reduction, they proposed that NfsB underwent a conformational change after substrate binding. This is a possibility, and some conformational changes are apparent upon binding of the cofactor (see Section 3.4.4 and Chapter 4 for more details); however, the $\mathrm{Km}$ value is not a reliable indicator for the comparison of interactions between substrates and an enzyme (Plesner, 1986). It is the $\mathrm{k}_{\mathrm{cat}} / \mathrm{Km}$ ratio, or catalytic specificity, that is the critical parameter for the comparison of substrate specificity (Copeland, 2000), and thus it is a variable slope on the primary double reciprocal plot that can signify a difference in substrate-enzyme interactions and reaction mechanisms. In fact, different $\mathrm{Km}$ values are observed for various cofactors and substrates of NEC while their $\mathrm{k}_{\mathrm{cat}} / \mathrm{Km}$ values are similar to one another, which suggests that this value is independent of the other substrate (Koder and Miller, 1998b). Conformational changes after cofactor binding in NfsB may account for the difference in $\mathrm{Km}_{\mathrm{NF}(\mathrm{NADPH})}$ and $\mathrm{Km}_{\mathrm{NF}(\mathrm{NADH})}$, as changes to the $\mathrm{FMN}$ binding pocket upon the binding of the nicotinamide analogue NIO are observed (Loverling et al., 2001; Chapter 4 this thesis). Another explanation could be that the reaction mechanism of $\mathrm{NfsB}$ is actually terreactant, with two of the substrates being identical (i.e. NADH or NADPH) (refer to Section 3.4.1.3, Figure 3.17).

The present study appears to be the only one that has compared the cofactor catalytic specificities of $\mathrm{NfsB}$, but in accordance with a previous study that compared specific activities (Bryant et al., 1981), the catalytic specificities established here 
demonstrate that wild-type $\mathrm{NfsB}$ has a slightly greater specificity for NADH than for NADPH. Figure $3.18(a-c)$ reveals a slight preference for NADH, which is primarily due to a lower $\mathrm{Km}$ value for nitrofurazone when NADH is used as a cofactor (refer to Table 3.4).

\subsubsection{Mutagenesis}

Targeted PCR mutagenesis was used in this study to evaluate the association of seven amino acids in NfsB, Gly-153 to Leu-159, with NAD(P)H. Another mutation at residue Phe-70 was fortuitously introduced by PCR: base substitutions occur at a frequency of approximately $1.1 \times 10^{-4}$ base substitutions per base pair with $\mathrm{Taq}$ polymerase (Tindall and Kunkel, 1988). This mutagenesis approach has the advantage of simultaneously obtaining numerous unique mutations within the target region. Thus it avoids the more costly and perhaps more time-consuming alternative approach of introducing each mutation independently. For instance, 14 unique mutations were analyzed in this study, which in a traditional site-directed mutagenesis strategy would have meant the use of 13 more primers to achieve the same number of mutations. The introduction of multiple mutations can be viewed as both an advantage and a disadvantage. A possible advantage is that it allows analysis of combinations of mutations; however this often represents a disadvantage since the majority of multiple mutations lead to enzyme inactivity and thus selection of single mutations becomes more time-consuming. Furthermore, two key problems were encountered in the development of this mutagenesis strategy. First, when only a single degenerate primer was used to incorporate mutations, mostly wild-type genes were obtained. Second, inefficient 
restriction endonuclease cleavage and ligation led to inaccurate subclones of the mutant genes. The latter problem was solved by the introduction of an intermediate cloning step into a pUC derived vector, which eliminated the need to use the $\operatorname{SapI}$ restriction endonuclease.

When this study was in progress a structure for E. coli $\mathrm{NfsB}$ was not available, so selection of the target region was primarily based on some similarity with the most commonly known consensus sequence for NAD-binding, the GXGXXG motif (Wierenga et al., 1985). The first glycine of this motif is highly conserved, but in some cases the second glycine has been replaced by valine, serine and alanine (Carugo and Argos, 1997). In $E$. coli $\mathrm{NfsB}$ an alanine is substituted for the second glycine. The substitution of an alanine at this position has been associated with effects on the binding of the adenine ribose (Rescigno and Perham, 1994). In E. cloacae NEC the complete GXGXXG sequence is present, which has been noted by other researchers (Bryant et al., 1991), although studies have not confirmed any involvement with $\mathrm{NAD}(\mathrm{P}) \mathrm{H}$ binding. These residues also align with the unverified $\mathrm{NAD}(\mathrm{P}) \mathrm{H}$ binding region in FRaseI, even though the two protein sequences are dissimilar at this location (Figure 3.19; an alignment of the proteins can be seen in Chapter 4, Figure 4.1) (Koike et al., 1998). Interestingly, shortly after this study was completed, the SWISS-PROT database (ExPASy) listed the 153-158 residue region of $\mathrm{NfsB}$ as the $\mathrm{NAD}(\mathrm{P}) \mathrm{H}$ binding site 'by similarity'. It is not clear what it has been compared to; as motif searches by PSITE (Solovyev and Kolchanov, 1994) and tools within the PredictProtein server (Rost, 1996), including ProDom (Corpet et al., 2000) and ProSite (Bairoch et al., 1997) do not identify this region (or any other in NfsB) 
as a potential binding site. Akin to the reasoning that motivated the mutagenesis approach used in this study, the identification was probably founded on similarity to the most commonly cited GXGXXG motif.

\subsubsection{Residues in target region may influence NADPH utilization}

The mutagenesis target region spanning residues Gly-153 to Leu-159 does conform to the imprecise description of an $\mathrm{NAD}(\mathrm{P}) \mathrm{H}$ binding site, but the 3D structure of NfsB (Parkinson et al., 2000) demonstrates that these residues are unlikely to come into contact with any substrate of the enzyme, and that they are not in direct contact with bound nicotinic acid (NIO) (Loverling et al, 2001). However, the present study demonstrates that mutations introduced in this region do affect $\mathrm{NfsB}$ catalysis and cofactor preference. Two key explanations may account for these apparent incongruities. First, NADPH differs from NADH solely by the presence of the 2' phosphate on the AMP moiety, which is not present in nicotinic acid, and thus the NIO-bound structure of $\mathrm{NfsB}$ is not a complete representation of the cofactor contacts. Second, many residues in an enzyme can modulate catalysis without any actual direct contact, for example, by long-range electrostatic effects and repositioning of residues at the reaction centre (Mittl et al., 1994; Plapp, 1995), so it is possible that this region is directly involved in catalysis. Indeed, the contributions of distant residues to the active site of $E$. coli glutathione reductase were demonstrated by the switch in cofactor dependence from $\mathrm{NADP}^{+}$to $\mathrm{NAD}^{+}$through the introduction of seven point mutations (Scrutton et al., 1990), which were as distant as $15 \AA$ from the actual cofactor (Mittl et al.,1993,1994). Of course, any missense mutation will result in some level of local structural change, so analysis of 
mutations must make a distinction between gross/indirect structural changes and direct effects on enzyme catalysis.

Most missense mutations examined within the target region resulted in the elimination of NR activity. The tempting implication is that this region is crucial for enzyme activity, but while this may be true, it may also be an oversimplification. It is possible that the amino acid substitutions made here were too drastic and that other substitutions could result in an active enzyme. Even though any amino acid change can potentially affect enzyme structure and/or catalysis, some interchanges are relatively conservative. A mutation data matrix compiled by Dayhoff et al. (1983), derived from comparisons of homologous proteins and consistent with chemical intuition, illustrates how some amino acid substitutions are relatively more frequent, or evolutionarily accepted, than others. According to this matrix, most of the mutations acquired in this study are usually not evolutionarily accepted (see Table 3.8 ). The exceptions are the Gly to Ser (positions 153 and 158) and Ala to Pro (positions 155 and 156) mutations. Indeed, the two Gly to Ser mutations obtained in this study, G153S and G158S, yielded the only mutant proteins that retained measurable NR activity. It is not surprising that the Ala to Pro substitutions disrupted NfsB function despite their general evolutionary acceptance. A proline substitution considerably decreases the carbon backbone's flexibility, which in an $\alpha$-helix may result in major structural change. This structural modification is possibly 
Table 3.8 Evolutionarily accepted amino acid substitutions based upon the mutation data matrix compiled by Dayhoff et al. (1983). Those residues in bold represent the two types of missense mutations that were obtained in this study. Only the Ser substitution resulted in an active enzyme, as discussed in the text.

\begin{tabular}{cc}
\hline Target residue & Frequently accepted mutation \\
\hline Gly & Ala, Ser \\
Val & Ile, Leu, Met \\
Ala & Ser, Gly, Thr, Pro \\
Leu & Met, Ile, Val, Phe \\
Phe & Tyr, Leu, Ile \\
\hline
\end{tabular}


the reason for the distinct shape of the A156P FMN fluorescence spectrum (Figure $3.8 \mathrm{~b}$, Table 3.3 ), the only enzyme for which peak I is smaller than peak II. The diminished NR activity of G153S and G158S suggests that these residues are directly involved in enzyme catalysis. Unfortunately, the NR activity of G153S is too low to allow kinetic analysis (see Section 3.3.4.3), but analysis of the other mutants with mutations in the target region, G158S and VAFS, demonstrated that this region apparently has an effect on NADPH usage.

The significantly decreased NADPH preference of G158S and VAFS is reflected in the low $\mathrm{k}_{\mathrm{cat}} / \mathrm{Km}$ ratios for this cofactor, about $50 \mu \mathrm{M}^{-1} \mathrm{sec}^{-1}$, compared to over $150 \mu \mathrm{M}^{-1} \mathrm{sec}^{-1}$ for NADH (Figure 3.18b). This effect is primarily due to the increased $\mathrm{Km}$ values for NADPH (Table 3.4), an indicator of decreased affinity, which is interesting as NADP coenzymes are usually more flexible than NAD in their complexes with enzymes. In fact, few enzyme/cofactor interactions are uniquely associated with NADPH (Carugo and Argos, 1997a). Despite numerous studies, a general explanation for NAD/NADP discrimination does not exist. There are some cases in which a loop between an $\alpha$-helix and $\beta$-strand has been shown to interact with the diphosphate moiety of NADP, with the dipole of the $\alpha$-helix thought to help stabilize the interaction (Carugo and Argos, 1997b). Gly-158 may participate in this type of interaction as it is located in the loop region between $\alpha \mathrm{G}$ and $\beta 3$ (refer to Figure 3.19), disruption of linkages involving Gly-158 could lead to decreased affinity for NADPH. Structural alterations here may disturb contact between other residues and the cofactor; the disruption of structural contacts is more thoroughly explored in Chapter 4. 
The Trp fluorescence spectrum of G158S shows evidence of some structural changes in the mutant enzyme: the low intensity $375 \mathrm{~nm}$ peak emitted by this mutant is shifted markedly from the $345 \mathrm{~nm}$ peak apparent for wild-type NfsB (Table 3.3). However, the FMN spectrum is similar to that of the wild-type indicating an undisturbed environment near the FMN molecule. Thus, the Trp spectrum probably reflects changes at the 2 residues (W46 and W94) that are furthest from the putative substrate binding site, rather than at the W138 residue that is near the FMN binding pocket. As the FMN spectrum of G158S reflects no major conformational changes, it seems likely that Gly158 , and/or residues influenced by a mutation at Gly-158, is involved in NADPH utilization. A more detailed examination of the structural effect of G158S on NfsB is included in Chapter 4. The structure of NfsB is shown in Figure 3.21 with the residues discussed in this chapter indicated in orange. Modeling of the mutant structures using SwissPDBviewer suggests that the Val to Ala substitution of VAFS does result in some disruption of the wild-type structure. Unlike G158S, the Trp fluorescence spectrum of VAFS is similar to the wild-type and does not have a peak at $375 \mathrm{~nm}$ (Table 3.3), but the FMN fluorescence spectrum exhibits significantly greater intensity than the other enzymes, especially for peak I (Figure 3.8b, Table 3.3). This increased fluorescence intensity is probably indicative of a structural disturbance in the mutant enzyme, which is not surprising considering that the V154A mutation alone significantly disrupts NR activity. Indeed, the spectrum of V154A indicates a structural disturbance near FMN, which is best reflected by the increase in the intensity of peak II compared to peak I (see Table 3.3 and Figure 3.8c). The fluorescence data obtained for the other mutant with no 
a.

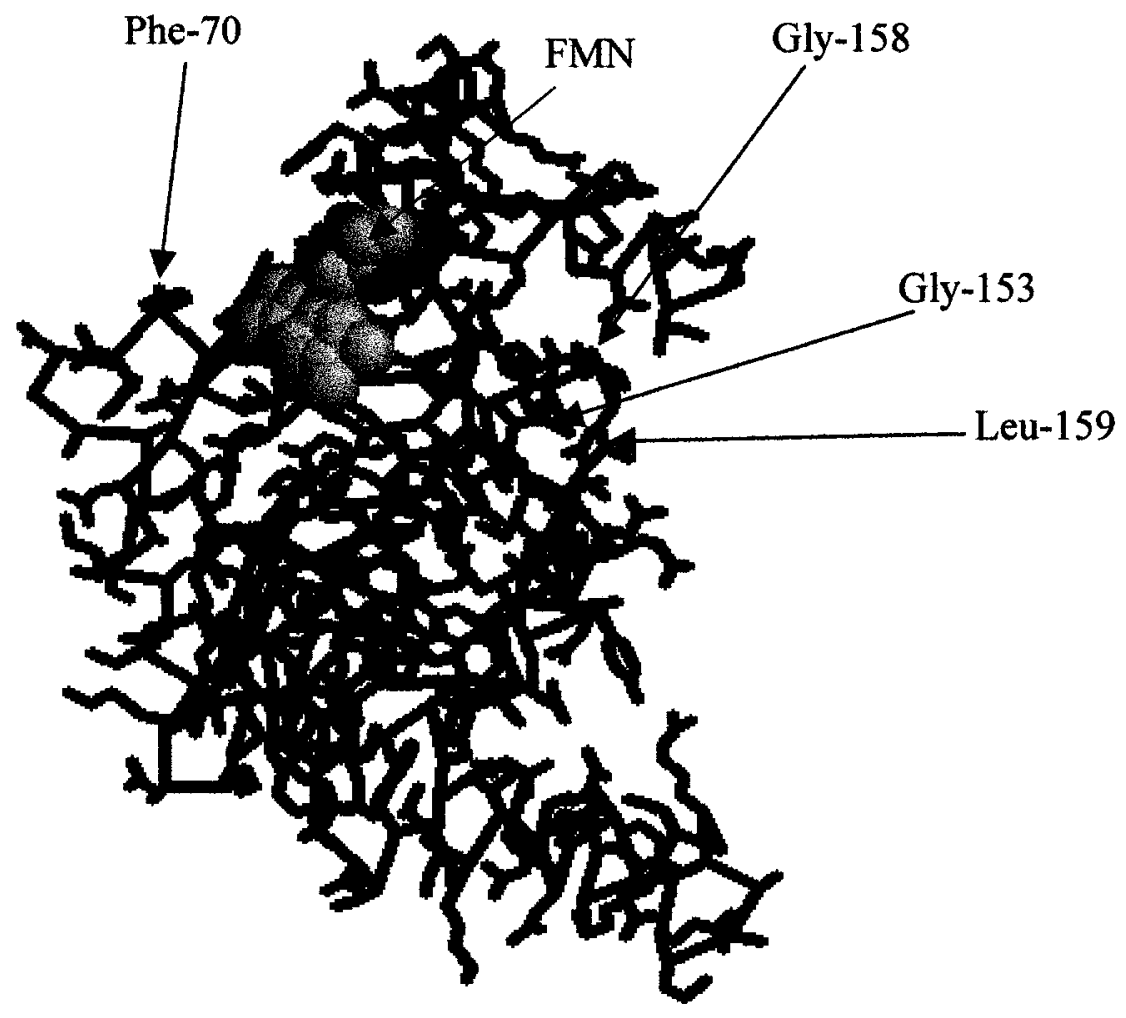

b.

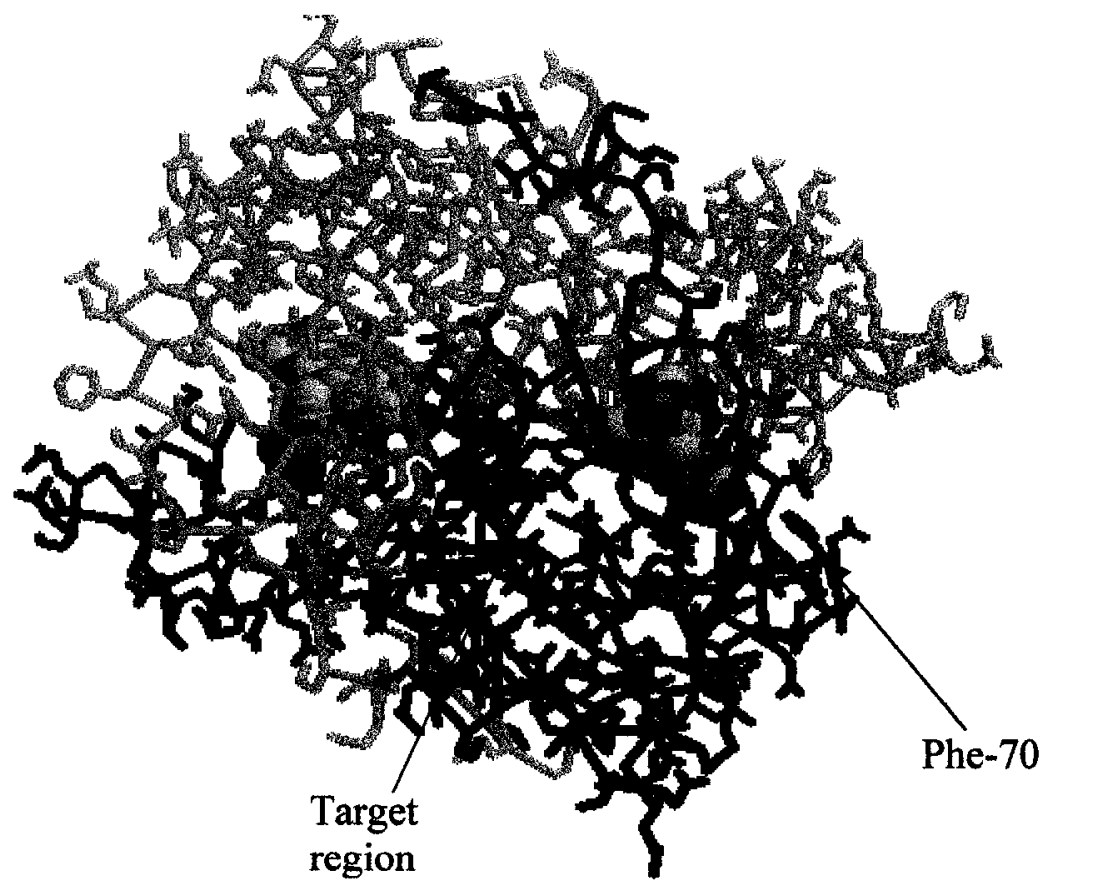

Figure 3.21 NfsB structure. Phe-70 and the mutagenesis target region from Gly-153 to Leu-159 in one chain of the homodimer structure are shown in orange. (a) Monomer of NfsB. (b) The NfsB homodimer. 
observed NR activity, A156P, indicates that FMN is bound in this enzyme, although in a different environment, as peak II exhibits a slight red shift to $555 \mathrm{~nm}$. Spectra were only obtained for those enzymes included in Table 3.3 and Figure $3.8 \mathrm{~b}$ as protein concentrations of other mutants were too low for analysis.

The turnover numbers of G158S are significantly less (about half) than for the wild-type. This could indicate an increase in the transition state energy for catalysis (Plapp, 1995; Rescigno and Perham, 1994), perhaps due to incorrect orientation of electron transfer resulting from distorted substrate binding. Small decreases in $\mathrm{Km}_{\mathrm{NADH}}$ and $\mathrm{Km}_{\mathrm{NF}(\mathrm{NADPH})}$ for $\mathrm{G} 158 \mathrm{~S}$ are evident. These may not be signs of increased affinity, but rather may reflect that those reactions of the ping-pong mechanism have become less rate limiting due to the decreased catalytic velocity (Vmax) with the other substrate(s) (Rescigno and Perham, 1994). This may also explain the electron acceptor dependent discrepancy in the $\mathrm{Km}_{\mathrm{NADH}}$ values for NfsB observed by Zenno and coworkers (1996b). In that study, the $\mathrm{Km}$ value for NADH was much lower with FMN $(5.4 \mu \mathrm{M})$ than with nitrofurazone $(22 \mu \mathrm{M})$ as the electron acceptor.

It is plausible that nitrofurazone exerts substrate inhibition in G158S, and even wild-type NfsB (Figure 3.19). Competitive inhibition by a substrate is not uncommon in ping-pong reactions as the slightly altered forms of the enzyme can often accommodate a substrate out of reaction order (Segel, 1975). Furthermore, the physiological substrate of $\mathrm{NfsB}$ is not known; therefore, while nitrofurazone may cause inhibition, it is unlikely that the natural substrate would, especially at physiological concentrations. However, 
experiments with higher concentrations of nitrofurazone would be necessary to confirm any such inhibition.

\subsubsection{F70S has no effect on cofactor specificity}

A Phe to Ser substitution is evolutionarily an infrequently accepted mutation (Dayhoff, 1983), and yet the F70S mutant exhibits full NR activity, although with altered kinetic characteristics. Unlike the residues in the target region, Phe-70 is in proximity to the putative substrate binding site and does have contact with four atoms of the bound FMN molecule (Lovering et al., 2001; Parkinson et al., 2000), but not with nicotinic acid (Lovering et al., 2001). According to the 3D structure of NfsB, Phe-70 is adjacent to the entrance of the putative substrate binding pocket. Introduction of the F70S mutation into the 3D structure of NfsB with SwissPDBviewer indicates that by removing the bulky phenyl group of Phe and replacing it with the smaller Ser side chain, this area is sterically less restrictive (Figure 4.7), which may help accommodate substrates.

There is no apparent shift in cofactor specificity with the F70S mutant. Indeed, as expected for a ping-pong mechanism, the $\mathrm{k}_{\mathrm{cat}} / \mathrm{Km}$ values for all of the substrates are similar to the wild-type values. However, there is some variability in the $\mathrm{k}_{\text {cat }}$ and $\mathrm{Km}$ values. An apparent decrease in affinity for the cofactors is implied by greater $\mathrm{Km}$ values; and increased turnover numbers may reflect a decreased transition state energy. Interestingly, Phe-70 apparently shifts conformation upon binding of nicotinic acid (NIO) (refer to Chapter 4, Section 4.4.2). It appears that a shift of Phe-70, and of Phe-124, opens an access channel (opening B in Figure 4.5) to the main active site pocket. In the NIO-bound structure Phe-70 only maintains contact with one of the four original FMN 
contacts. Figure 4.7 illustrates that the substitution of Ser at this location mimics the shift of the aromatic sidechain of Phe-70, which possibly facilitates access to the main pocket from this direction. This could be the reason for the increase in turnover number of the F70S mutant enzyme, as well as for the decrease in affinity for the cofactors since more conformations (e.g. both oxidized and reduced) could bind. 


\section{CHAPTER FOUR: COMPUTER ANALYSIS OF THE NFSB ACTIVE SITE \\ Implications for structure-based drug development.}

\subsection{Introduction}

Enzymes bind and orient substrates in an active site to facilitate a chemical transformation. Composed of amino acid residues that modulate activity of the enzyme, the active site includes a reaction centre as well as residues that are not in direct contact with the substrate(s) (Mittl et al., 1994; Plapp, 1995). The catalytic specificity of an enzyme is dependent upon multiple interactions that define the size and shape of the active site. An enzyme catalyzes a reaction by affecting the environment at this site; for example, by facilitating proton transfers or altering the electrostatic environment.

A goal of biotechnology is the ability to create a made-to-order enzyme, particularly for medicinal and pharmaceutical purposes. To date, large scale drug discovery efforts have focused less on redesign of enzymes with known structures, by site directed mutagenesis for example, than on de novo and rational design of biomolecules. A de novo approach attempts to form an biomolecule that folds and catalyzes a substrate within a specific environment (Blackburn, 2000), while rational design refers to a combinational chemistry approach that entails the selection of a particular enzyme from a pool of randomly generated ones using high-throughput screening techniques (Gane and Dean, 2000). Until recently, rational design was thought to be the most promising strategy for drug development (Amzel, 1998); however, with the technological advancements in biology and bioinformatics, structure-based drug development is now emphasizing the integration of clinical, cellular, biochemical, structural and biophysical knowledge of the target protein (Pattabiraman, 2002). The increasing ease and speed of 
protein structure determination has led to over 18,000 protein entries, and therefore potential drug targets, in the Protein Data Bank (there were about 7,000 in the year 2000) (www.rcsb.org), and the computational techniques to analyze these structures are also rapidly growing in number, accessibility, and utility (Joseph-McCarthy, 2002). The advent of the information age in biology thereby allows the rational redesign of an existing enzyme to be a viable option for large scale structure-based drug development (Blackburn, 2000). Indeed, the redesign of enzyme substrate and cofactor specificity based on 3D structure information is becoming more common and accurate (refer to Cedrone et al., 2000 for some impressive examples), and the future of drug development probably lies in the cooperation of knowledge-based enzyme redesign with combinational high-throughput screening methods (Cedrone et al., 2000).

The first step in structure-based drug development is the identification of functionally relevant binding sites on the target enzyme (Sotriffer and Klebe, 2002). This is facilitated by computational analysis, which can aid in the recognition of protein pockets by geometric analyses (Liang et al., 1998), docking scans for ligand complementarity (Gane and Dean, 2000; Sotriffer and Klebe, 2002), structure comparison, and similarity searches of protein cavities (Sotriffer and Klebe, 2002). With the increasing number of freely available programs, which are often powerful web-based applications, the ability to meaningfully analyze proteins for the purposes of drug development is becoming more accessible to most researchers; although docking programs and compound libraries are still largely restricted to industrial institutions (Gane and Dean, 2000). 
In this study, the active site of $\mathrm{NfsB}$ was analyzed with computational tools freely available from the Internet. The purpose of this study was two-fold: first, to elucidate key elements of NfsB's active site by means of computer analysis; second, to use this information to predict regions of $\mathrm{NfsB}$ that can be recruited for drug development. To achieve these goals, four primary research objectives were undertaken. First, the six available 3D structures from the nitroreductase family were obtained, and a structural alignment using $\mathrm{NfsB}$ as a template was generated. Second, protein pockets of these structures were identified and the structural contacts were determined. Third, a thorough structural comparison of NfsB and NfsA was accomplished. And fourth, the mutant structures of NfsB from the previous studies (Chapters $2 \& 3$ ) were modeled and compared with the wild-type structure. This information, combined with insights acquired in previous chapters of this thesis and from the literature, provides a more complete representation of the $\mathrm{NfsB}$ active site, forms the basis for enzyme redesign experiments, and reveals some promising targets for drug development. 


\subsection{Methods and Materials}

\subsubsection{Identification and visualization of $N f_{s} B$ homologues}

NfsB and five homologues (Table 4.1) were identified in the Structural Classification of Proteins (SCOP) database (http://scop.mrc-lmb.cam.ac.uk/scop) (Murzin et al., 1995) and the structural files (.pdb) were downloaded from the Protein Data Bank (PDB) (www.rcsb.org) (Berman et al., 2000). The 3D structures of the enzymes were visualized in Protein Explorer (Martz, 2000), obtained from http://proteinexplorer.org, unless otherwise noted. Protein Explorer requires the Chime plug-in that is available at http://www.mdlchime.com/chime/ (MDL Information Systems).

Several approaches were used to identify additional homologues of $\mathrm{NfsB}$ including a text search of the PDB with 'nitroreductase', a PSI-BLAST search (Altschul et al., 1997) with default parameters on the ConSurf server (http://bioinfo.tau.ac.il/ConSurf/) (Glaser et al., manuscript in preparation), and a search using the Combinatorial Extension method for protein structure comparison (http://cl.sdsc.edu/ce.html) (Shindyalov and Bourne, 1998). A 3D structure of NfsB complexed with NIO, and of Vibrio harveyi Flavin reductase $\mathrm{P}$ (Frp) bound to NAD ${ }^{+}$ were also downloaded for further analysis. Three resolutions $(1.7 \AA, 1.8 \AA$ and $2.4 \AA$ ) of the NfsB-NIO structure were available, so IICR $(1.7 \AA)$, the structure with the lowest Rfactor and best resolution, was used. 


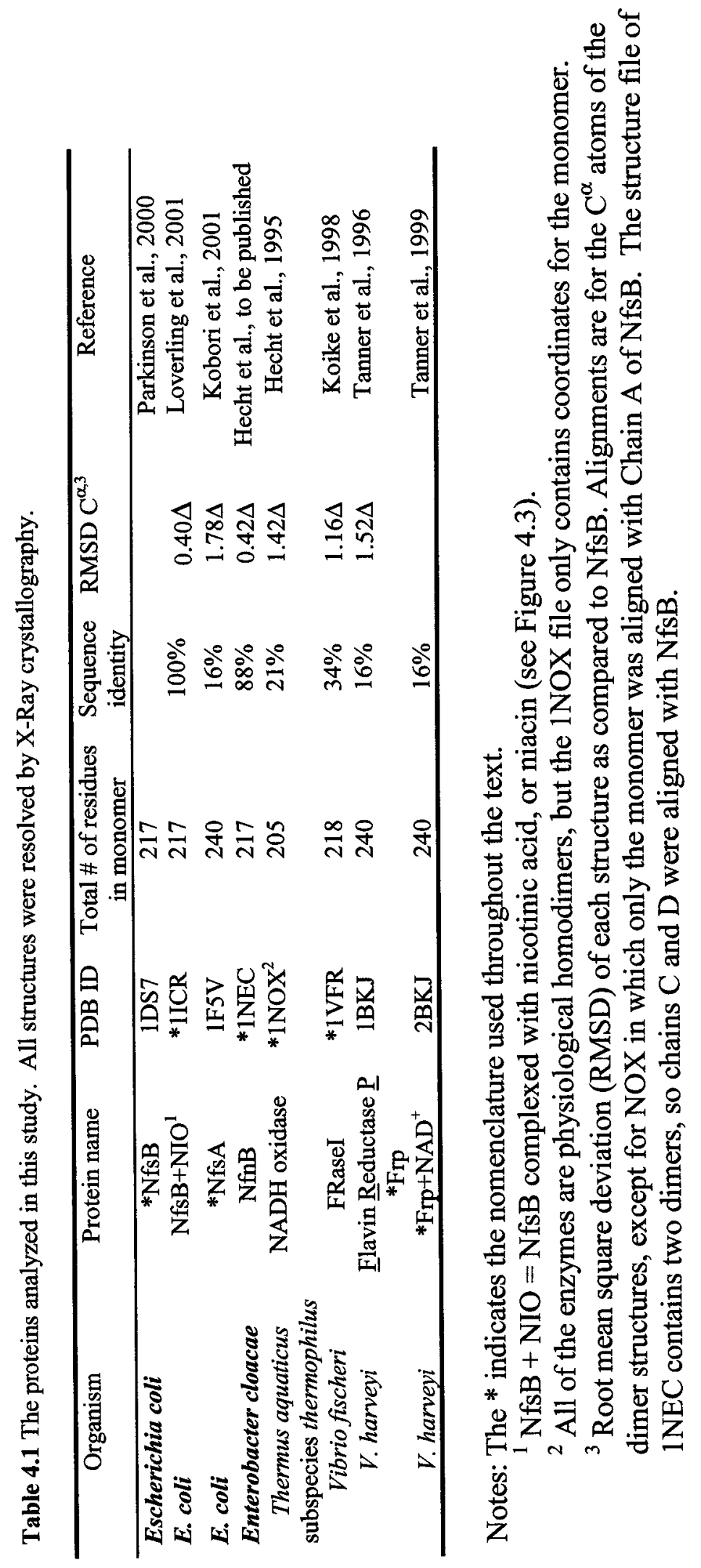




\subsubsection{Structural alignment}

A 3D alignment of NfsB with the other five structures was generated in SwissPdbViewer v.3.7b2, which was obtained from the ExPASy molecular biology server (http://www.expasy.ch/spdbv/) (Guex and Peitsch, 1997). NfsB was used as the template upon which each structure was independently aligned. A detailed description of this procedure is available in the tutorial material, indexed as 'superimposition', provided on the ExPASy site. Briefly, the PDB structure files were opened in the Swiss-PdbViewer v.3.7b2 program and the 'magic fit' tool was used to roughly align each structure with NfsB. The 'iterative magic fit' tool subsequently optimized each alignment, and a structural alignment was created with the 'generate structural alignment' tool. The alignments were viewed and manually adjusted as necessary. The root mean square deviation (RMSD) of each alignment was determined using the 'calculate RMS' option in the tools menu. The RMSD is calculated according to the following equation: $\left(\Sigma(\mathrm{dist})^{2} / \mathrm{nbAtoms}\right)^{1 / 2}$, where dist is the distance (in angstroms) between a pair of related atoms. The Swiss-PdbViewer program was also used to analyze the protein structures, including the determination of B-factors (tools menu).

\subsubsection{Determination of interatomic contacts}

The structures of the six NR family proteins were resolved with bound FMN. Identification of the FMN-protein contacts was accomplished with the Ligand-Protein Contact (LPC) program available on http://bioinfo.weizmann.ac.i1:8500/oca-bin/lpccsu (Sobolev et al., 1999). The contacts of nicotinic acid (NIO) in 1ICR, and of $\mathrm{NAD}^{+}$in $2 \mathrm{BKJ}$ were also determined in this way. The associated Contacts of Structural Units 
(CSU) software (Sobolev et al., 1999) identified contacts made by other residues in the proteins. Some contacts were also identified with the Swiss-PdbViewer and Protein Explorer programs.

\subsubsection{Identification of protein pockets and cavities}

Binding sites are often associated with structural pockets in a protein. The CASTp server (http://cast.engr.uic.edu/cast/) calculates exterior pockets and interior cavities of a protein, measures the area and volume of the pockets and the number and size of the pocket mouth openings based on geometric analyses (Liang et al., 1998). This server was used to identify the structural pockets of NfsB and the other enzymes.

\subsubsection{Modeling of mutations}

The mutants recovered in chapters 2 and 3 have mutations in NfsB that alter its NR activity. The missense mutations listed in Table 4.2 represent mutants described in previous studies; the substitutions were introduced into the 3D structure of $\mathrm{NfsB}$ using the mutation tool of Swiss-PdbViewer v.3.7b2, which selects the least stressful rotamer of the introduced residue. Analysis of the mutant enzymes was accomplished with the same programs described above for the wild-type enzymes. 
Table 4.2 NfsB mutations, and one NfsA mutation, that were modeled in this study. Mutations were based on the mutants obtained in previous studies. Alterations in the structural and ligand contacts of the mutant structures were assessed by comparison with the wild-type structure.

\begin{tabular}{|c|c|}
\hline Mutation $^{\mathrm{a}}$ & Source $^{\mathrm{b}}$ \\
\hline L33P & Ch. 2 \\
\hline L34R & Ch. 2 \\
\hline S37R & Ch. 2 \\
\hline $\mathrm{S} 40 \mathrm{C}$ & Ch. 2 \\
\hline $\mathrm{I} 42 \mathrm{Q}$ & Ch. 2 \\
\hline P45E & Ch. 2 \\
\hline *F70S & Ch. 3 \\
\hline V83D & Ch. 2 \\
\hline D105Y & Ch. 2 \\
\hline *F124S & Zenno et al., 1996c \\
\hline G148S & Ch. 2 \\
\hline G153S & Ch. 3 \\
\hline G153R & Ch. 3 \\
\hline $\mathrm{G} 153 \mathrm{C}$ & Ch. 3 \\
\hline *V154A & Ch. 3 \\
\hline V154E & Ch. 3 \\
\hline V154G & Ch. 3 \\
\hline A155S & Ch. 3 \\
\hline A155P & Ch. 2 , Ch. 3 \\
\hline A156P & Ch. 3 \\
\hline L157Q & Ch. 3 \\
\hline L157R & Ch. 3 \\
\hline L157P & Ch. 3 \\
\hline${ }^{*} \mathrm{G} 158 \mathrm{~S}$ & Ch. 3 \\
\hline L159Q & Ch. 3 \\
\hline Y183D & Ch. 2 \\
\hline *V154A \& F70S & Ch. 3 \\
\hline$* \mathrm{E} 99 \mathrm{G}(\mathrm{NfsA})^{\mathrm{c}}$ & Zenno et al., 1998a \\
\hline
\end{tabular}

${ }^{a}$ The number indicates the residue number in the protein (refer to Figure 4.1). The first letter is the wild-type amino acid and the second letter is the one that is substituted in the mutant enzyme. See Appendix I for amino acid codes.

${ }^{b}$ Indicates where this mutant was originally obtained and studied. Ch. 2 and Ch. 3 refer to Chapters 2 and 3, respectively, of this thesis.

${ }^{c}$ This mutation was obtained in $E$. coli NfsA.

* These mutant structures were analyzed in more detail, particularly with the CASTp program to determine protein pockets and cavities. 


\subsection{Results}

\subsubsection{Structural alignment of $N f s B$ and its homologues}

Alignments of primary protein sequences do not always reflect structurally conserved regions in proteins. This is particularly true in the case of NAD(P)H binding sites, which often share a conserved structural domain despite little sequence similarity (Carugo and Argos, 1997a,b). A principal division in the nitroreductase family is between the NfsA-like enzymes that exclusively use NADPH as a cofactor (NfsA, Frp), and the NfsB-like enzymes that can use both NADH and NADPH as cofactors (NfsB, VFR, NEC and NOX). A structural comparison of the six protein structures that have been resolved in the nitroreductase family can thus lead to a more complete conceptualization of the NfsB active site; and a comparison of the NfsA-like and NfsBlike enzymes can provide an indication of which elements might be important for $\mathrm{NAD}(\mathrm{P}) \mathrm{H}$ discrimination.

The structural alignment of one monomer from $\mathrm{NfsB}$ and its five homologues is shown in Figure 4.1. All of the enzymes are physiological homodimers, although the resolved T. thermophilus NOX structure is for a monomer. As expected for homologous enzymes, all of the alignments yielded RMSD values of less than $2 \AA$ (Gille et al., 2000; Rost et al., 1997). Not surprisingly, the largest deviations, $1.78 \AA$ and $1.52 \AA$, are for the NADPH dependent enzymes, NfsA and Frp, respectively.

\subsubsection{NfsB: Protein pockets and structural contacts}

The CASTp program calculated 61 pockets and cavities within the NfsB dimer structure, 30 per monomer plus one pocket (IV) (Table 4.3, Figure 4.2) that is 
Figure 4.1 Structural alignment of NfsB and homologues. See Table 4.1 for protein identifiers, and refer to the text for details. Elements of secondary structure for $\mathrm{NfsB}$ are identified above the protein sequence as boxes (grey for $\alpha$-helices, black for $\beta$-strands). These are nearly identical for the NfsB homologues, including NfsA and Frp. The differences for NfsA are shown below the protein sequences as white boxes with dotted outlines (see also Figure 4.9). The $\beta$-strands are the same size and in the same location in all of the proteins. Bold $=$ in major pocket; $\underline{\text { single underline }}=\mathrm{FMN}$ contact; $\underline{\underline{\text { double }}}$ $\underline{\underline{\text { underline }}}=\mathrm{NIO}$ or $\mathrm{NAD}^{+}$contact; bold italics $=$in major pocket, but has no observed contacts with FMN or nicotinamide cofactor. 


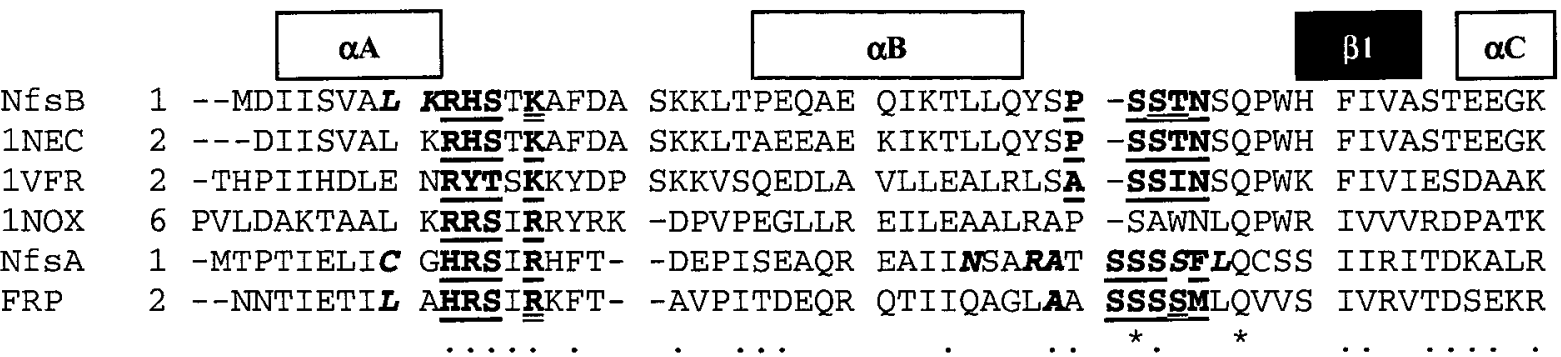

$\begin{array}{lll}\alpha \mathrm{C} & \beta 2 & \alpha \mathrm{D} \\ \alpha \mathrm{F}\end{array}$

NfSB 58 ARVAKSAAGN YVFNERKMLD ASHVVVFCAK TAMDDVWLKL VVDQEDADGR FATPEAKAAN

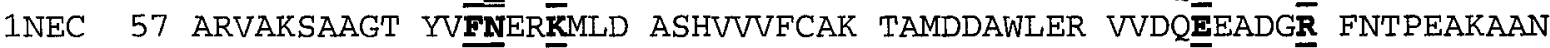

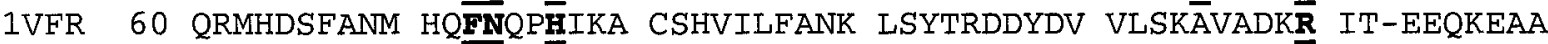

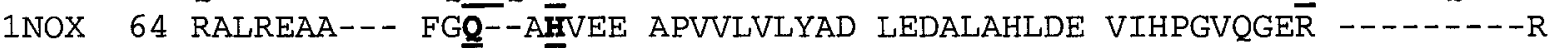

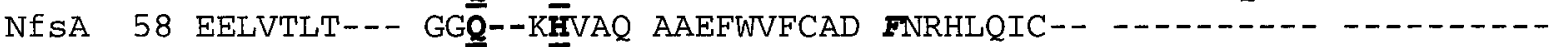

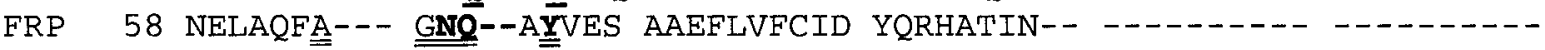
. . $\quad$. $\alpha \mathbf{E}$

\begin{tabular}{|c|c|c|}
\hline$\alpha \mathbf{F}$ & $\alpha \mathrm{G}$ & $\alpha 3$ \\
$\alpha \mathbf{H}$ \\
\hline
\end{tabular}

NfSB 118 DKGRKFFADM HRK-DLHDDA EWMAKQVYLN VGNFLLGVAA LGLDAVPIEG FD--AAILDA 1NEC 117 HKGRTYFADM HRV-DLKDDD QWMAKQVYLN VGNFLLGVGA MGLDAVPIEG FD--AAILDE

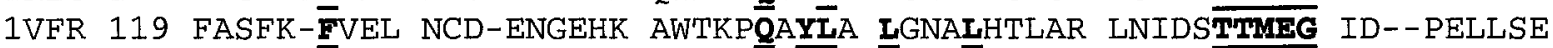
1NOX 110 EAQKQAIQRA FAAMGQEARK AWASGQSYYIL LGYLILLLEA YGLGSVPMLG FD--PERVRA NfSA 91 --.--P DAQLGLAEQL LLGVVDTAMM ÄQNA $\bar{L} I A A E S$ LGLGGVYIGG LRNNIEAVTK

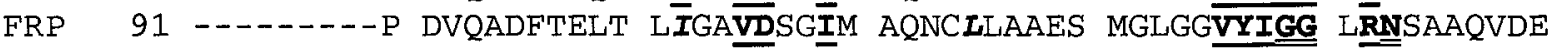

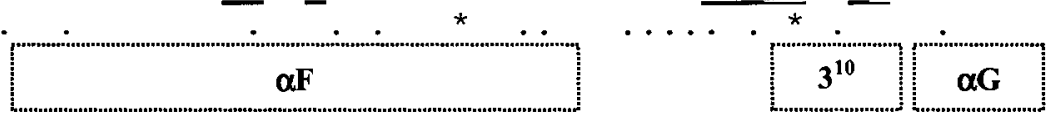

\section{$\beta 4$}

\section{$\alpha \mathbf{I} \beta 4$}

NESB 175 EFGLKEKGYT SLVVVPVGHH SVE-DFNATL FKSRLPQNIT LTEV

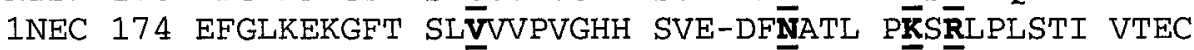

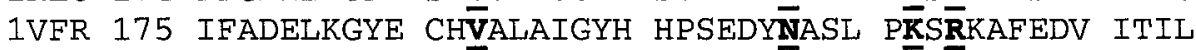

INOX 168 ILGLPSR-AA IPĀLVALGY

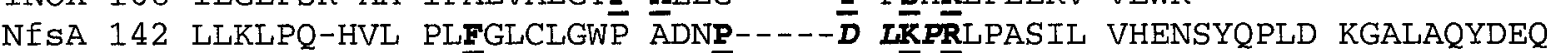

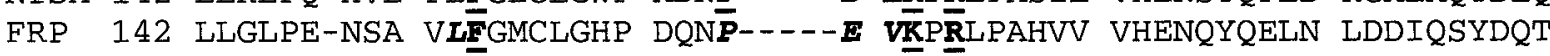
.*. .

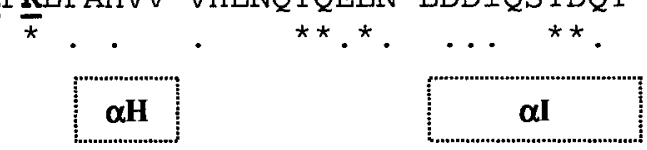

NfSA 196 LAEYYLTRGS NNRRDTWSDH IRRTIIKESR PFILDYLHKQ GWATR FRP 196 MQAYYASRTS NQRCSTWSQE VTGKLAGESR PHILPYLNSK GLAKR
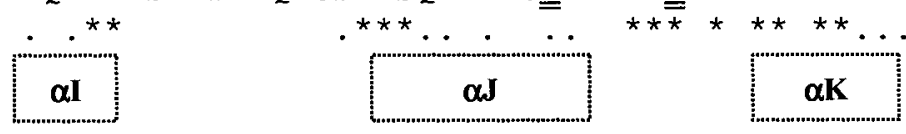


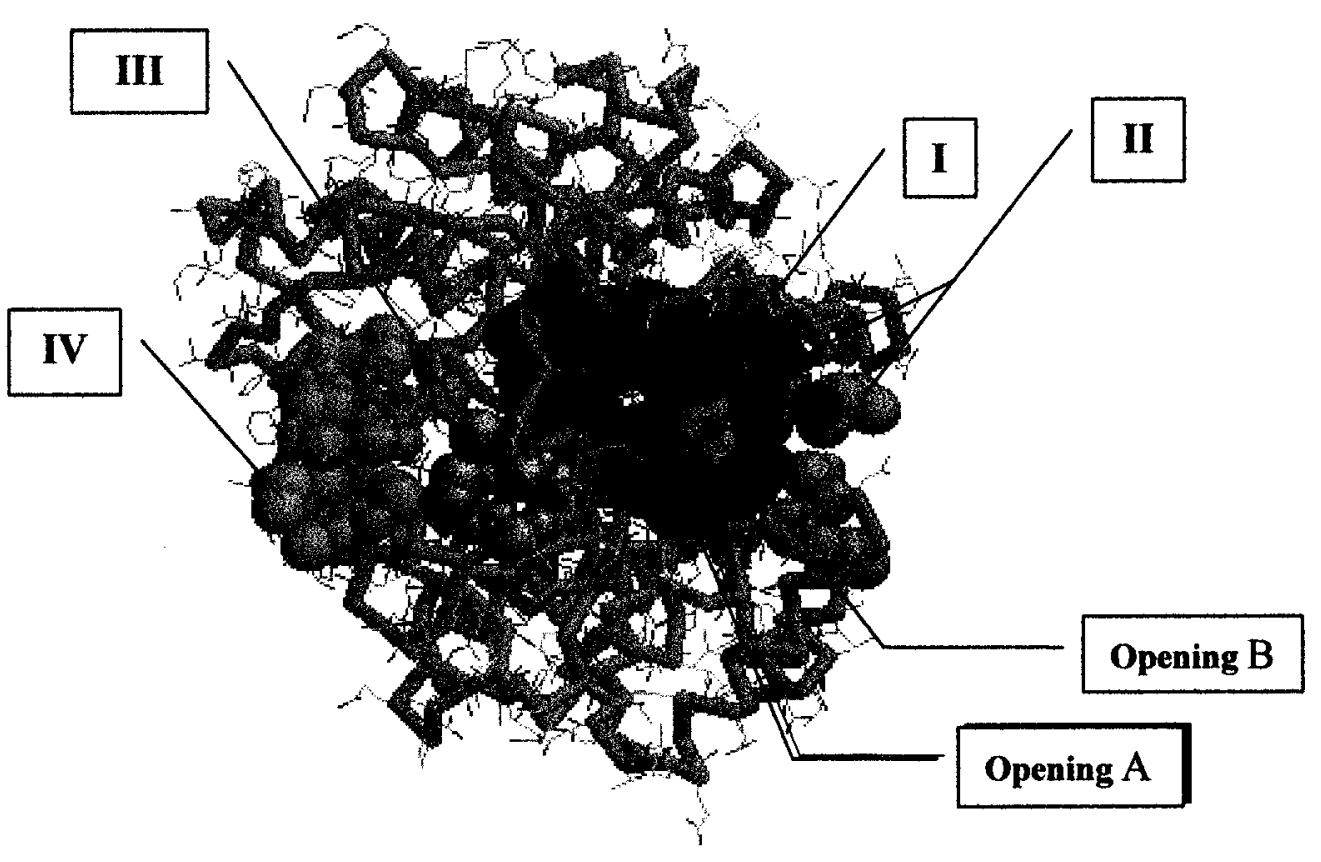

Figure 4.2 Solvent accessible pockets of NfsB from the perspective of 'looking' into mouth opening A of the major pocket (i.e. opening B is viewed within the major pocket through mouth opening A). Refer to Tables 4.3 and 4.4 for volumes and residue composition of the pockets. 


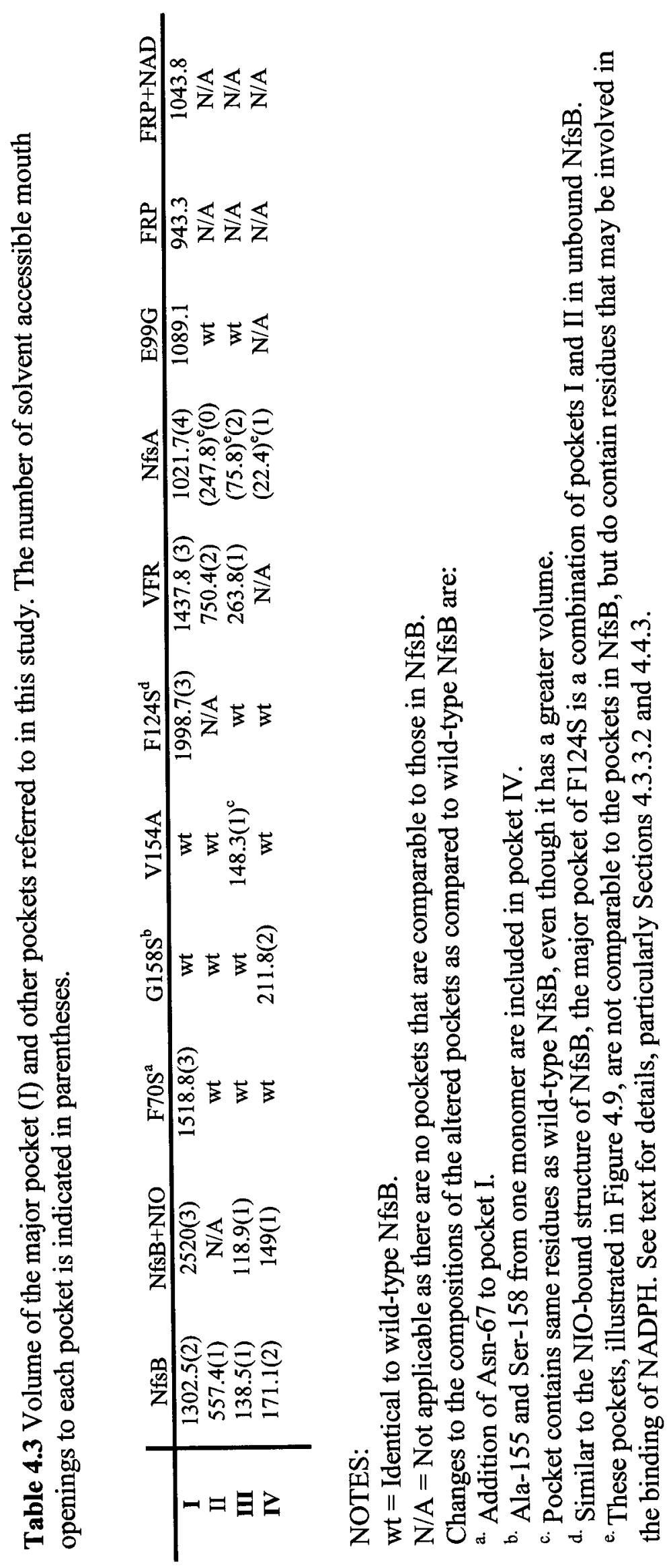


symmetrically composed of equivalent residues from each chain. Many of these pockets are small, with molecular volumes of less than $20 \AA^{3}$, but seven have volumes exceeding $100 \AA^{3}$. The largest pocket of NfsB and its homologues contains FMN and is thus designated the major pocket, while the other pockets are referred to as ancillary pockets. The CASTp data are considered within the context of other information including a comparison with the NIO-bound structure of NfsB, singular features of the structural alignment (Figure 4.1), especially with regards to NfsA (Section 4.3.3), and the experimental results obtained from previous studies, particularly the mutants analyzed in chapters 2 and 3 (Section 4.3.4).

\subsubsection{The major pocket: FMN, NAD(P)H and substrate binding}

All of the structures in this study were resolved with bound FMN (see Figure 4.3b for the structure of FMN). All of these enzymes are physiological homodimers, with the two bound FMN molecules positioned within crevices formed at the dimer interface (refer to Figure 4.4). In NfsB these two major pockets are V-shaped, hold the bound FMN at the narrow part of the cleft, and are similar to each other in volume and composition; therefore, unless otherwise noted, only one monomer will be referred to throughout this chapter. Access to the major pocket in NfsB is possible by means of one large (A) and one small (B) opening (Figure 4.5). Opening B is located between the major pocket (I) and pocket II (seen on Figures $4.2 \& 4.5$ ). The volume of the major pocket is approximately $1300 \AA^{3}$, which is noticeably smaller than in the F70S mutant $\left(1519 \AA^{3}\right)$, and much less than in NfsB complexed with NIO $\left(2520 \AA^{3}\right)$ (see Section 4.3.2.2.2) 


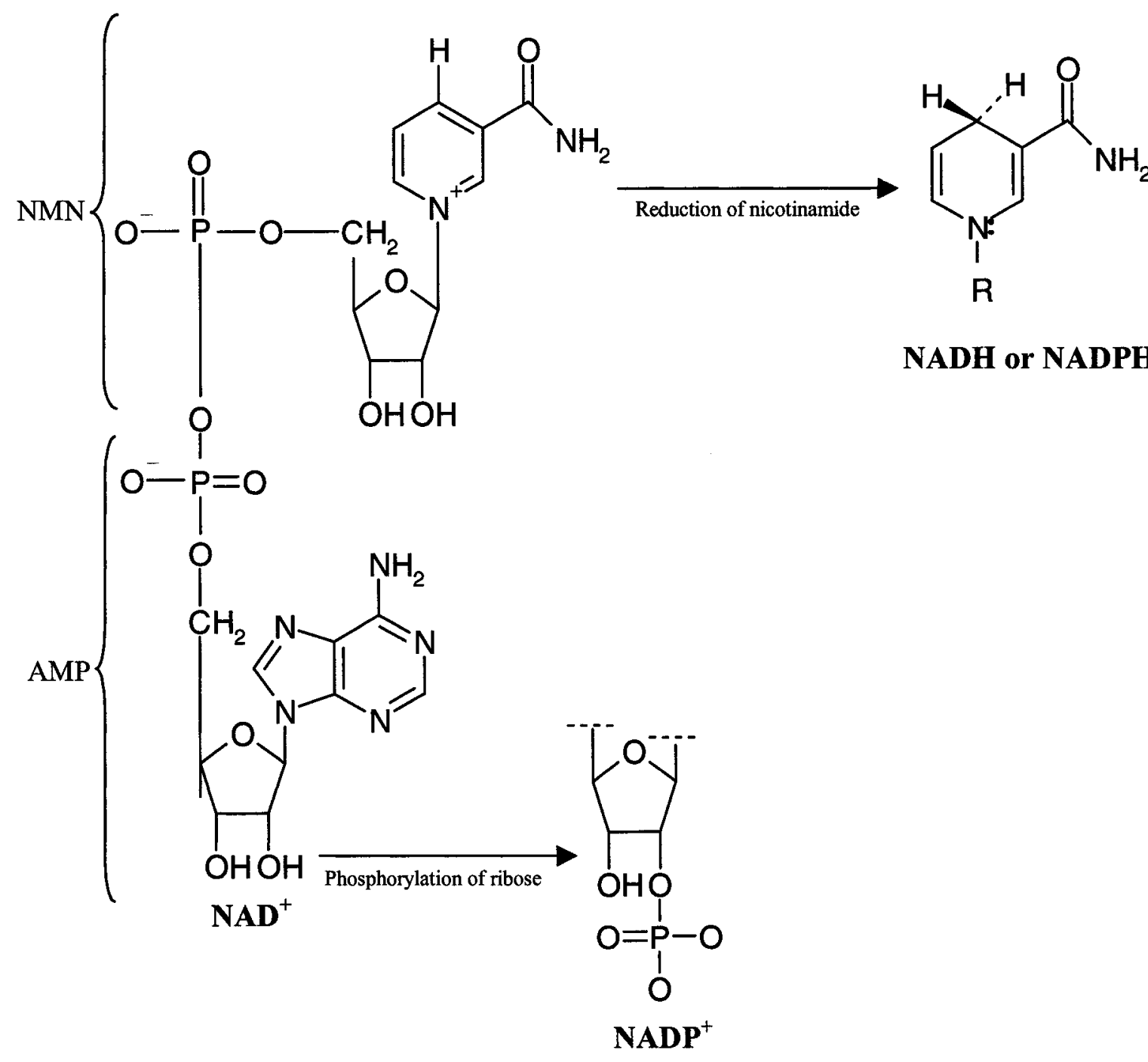

(a) NAD(P)H

Figure 4.3 Diagrams of the cofactor structures mentioned in this study. (a) Oxidized and reduced nicotinamide adenine dinucleotide $\left(\mathrm{NAD}^{+}\right)$and NAD phosphate $\left(\mathrm{NADP}^{+}\right)$; (b) FMN; (c-e) cofactor analogues. Continued on next page. 


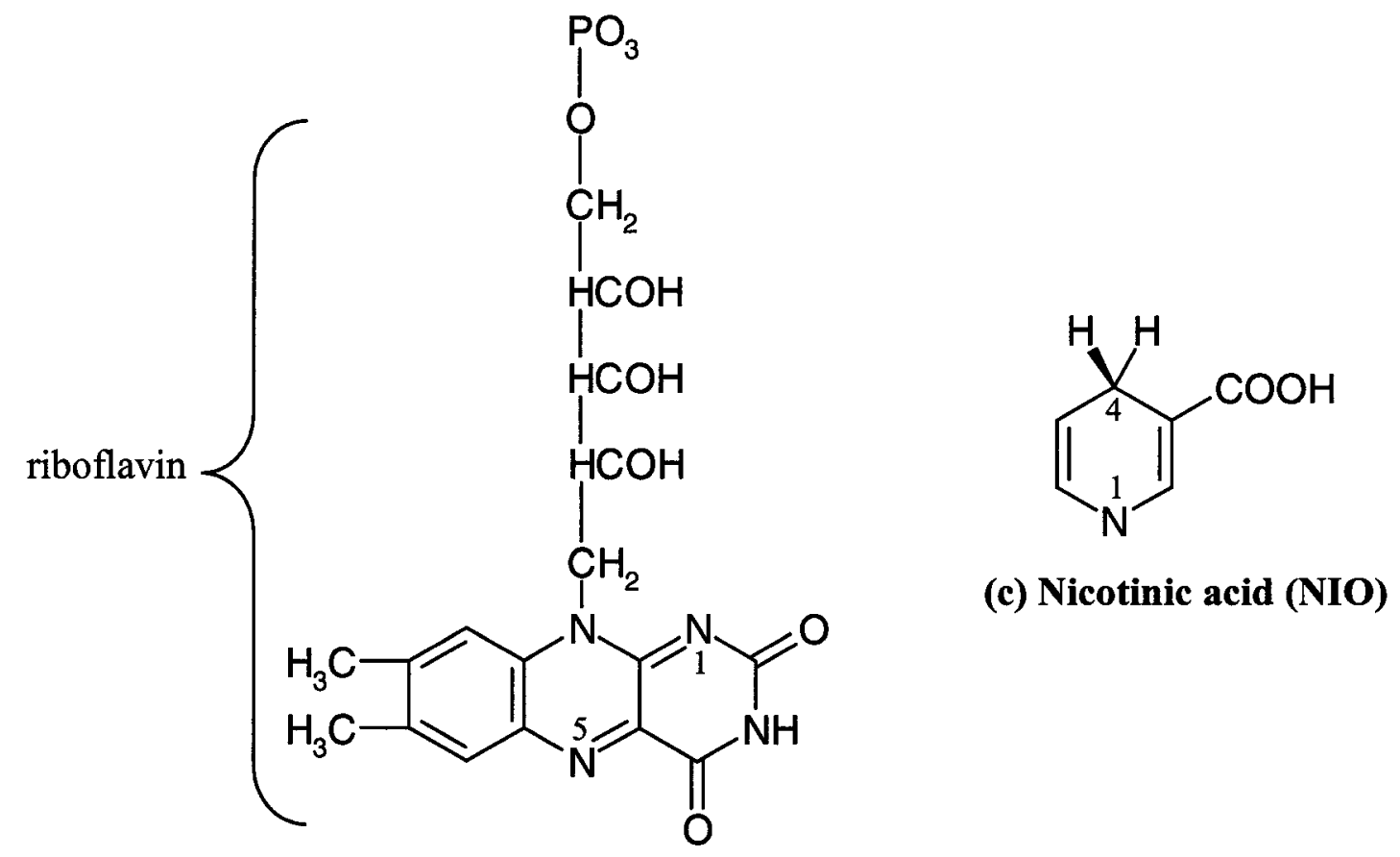

(b) Flavin mononucleotide

(FMN)<smiles>CN1C=CCC(C(N)=O)=C1</smiles>

(d) 1-methylnicotinamide

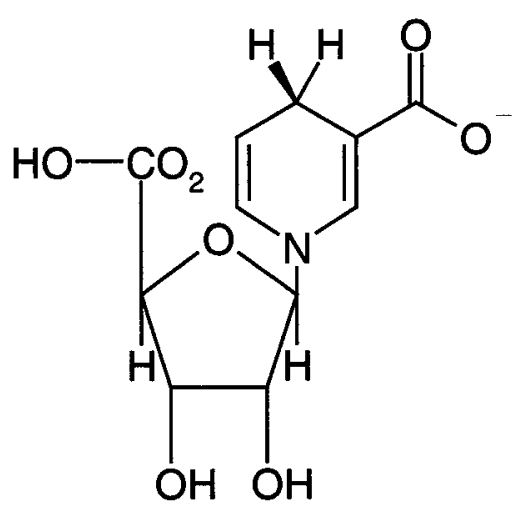

(e) Reduced nicotinic acid riboside (NARH)

Figure 4.3 (cont'd) 
Figure 4.4 Side and front views of the FMN-containing major pocket in (A) NfsB (top) and (B) NIO-bound NfsB (bottom). The two monomer chains are in different colours. For the ligands grey $=$ carbon atoms, red $=$ oxygen, blue $=$ nitrogen. 

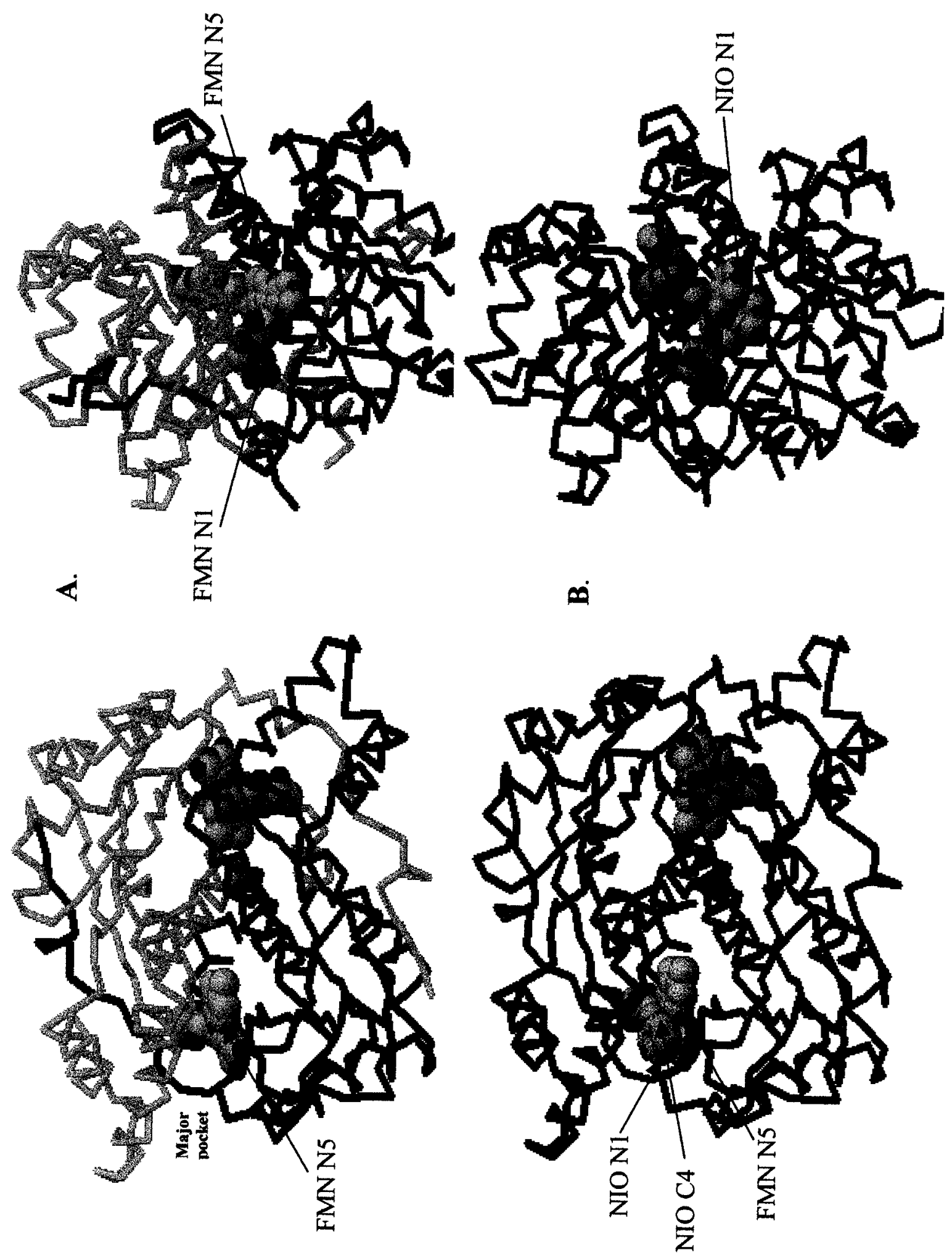


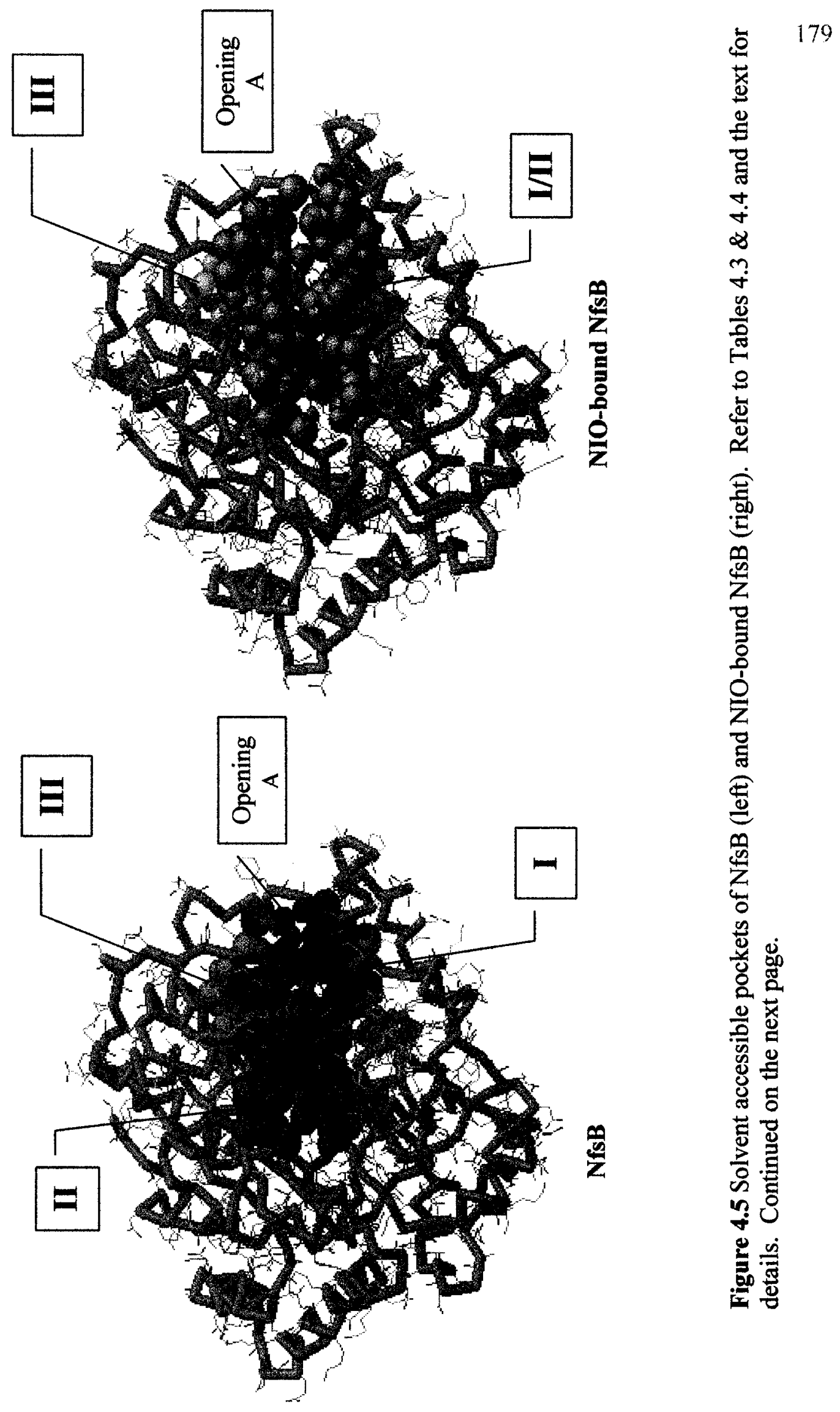




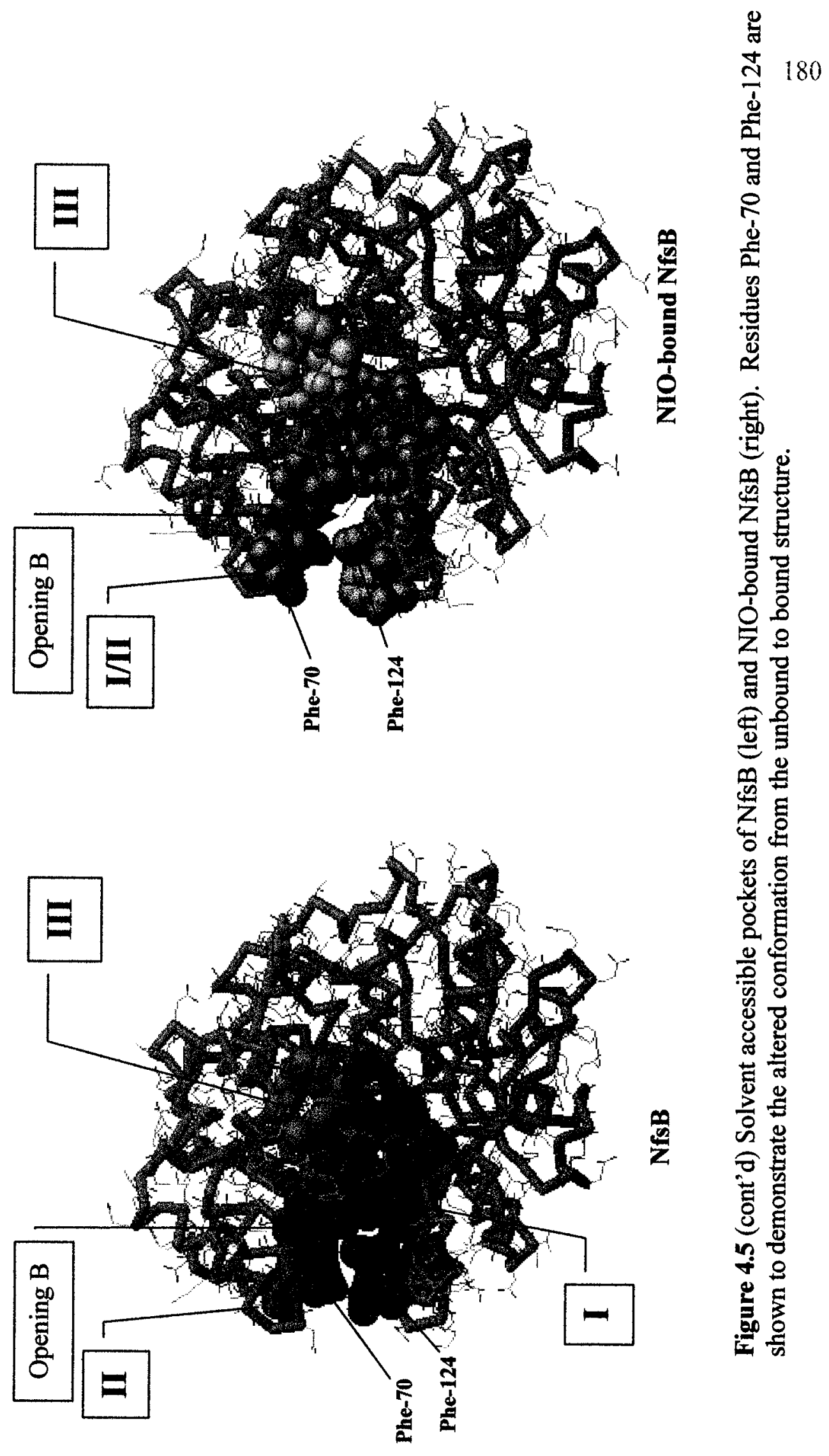


In $\mathrm{NfsB}$, all of the FMN contact residues reside within the major pocket, and the bound position and conformation of FMN is conserved in the homologous enzymes. FMN is in a butterfly conformation with the si face buried in the protein and the re face solvent accessible (see Figure 4.4). Parkinson et al. (2000) include a detailed description of the FMN binding pocket in NfsB and note that access to FMN is limited to the third ring and re face of the cofactor. The protein-ligand contacts derived with the LPC program (Sobolev et al., 1999) are underlined and in bold in Figure 4.1, while Figure 4.6a illustrates these contacts for NfsB. In Figure 4.1 all residues that are in contact with FMN are noted regardless of the contributing monomer, as only one monomer of the alignment is shown. Some of the apparently missing contacts in NOX on this figure are attributable to NOX being resolved as a monomer. Most noticeable is the absence of contact with Pro-38 to Asn-42 in NOX since these residues are contributed from the other monomer.

One structure of $\mathrm{NfsB}$ was resolved with $\mathrm{NIO}$, an analogue of the $\mathrm{NAD}$ cofactor (see structure in Figure 4.3c). The protein contacts of NIO are double underlined on Figure 4.1, and are illustrated in a LIGPLOT diagram in Figure 4.6b. Many of the nicotinamide contact residues in $\mathrm{NfsB}$ are also in contact with $\mathrm{FMN}$, as both cofactors are located within the major pocket of the enzyme. The exceptions are H128 and W128, both of which are located in pocket II (Table 4.4, Figure 4.2). In the NIO-bound structure of $\mathrm{NfsB}$, this pocket is incorporated into the major pocket (see Section 4.3.2.2.2 and Figure 4.5). However, while the NIO-bound structure contributes valuable information about cofactor binding in $\mathrm{NfsB}$, the entire cofactor is not represented (see Figure 4.3 for 


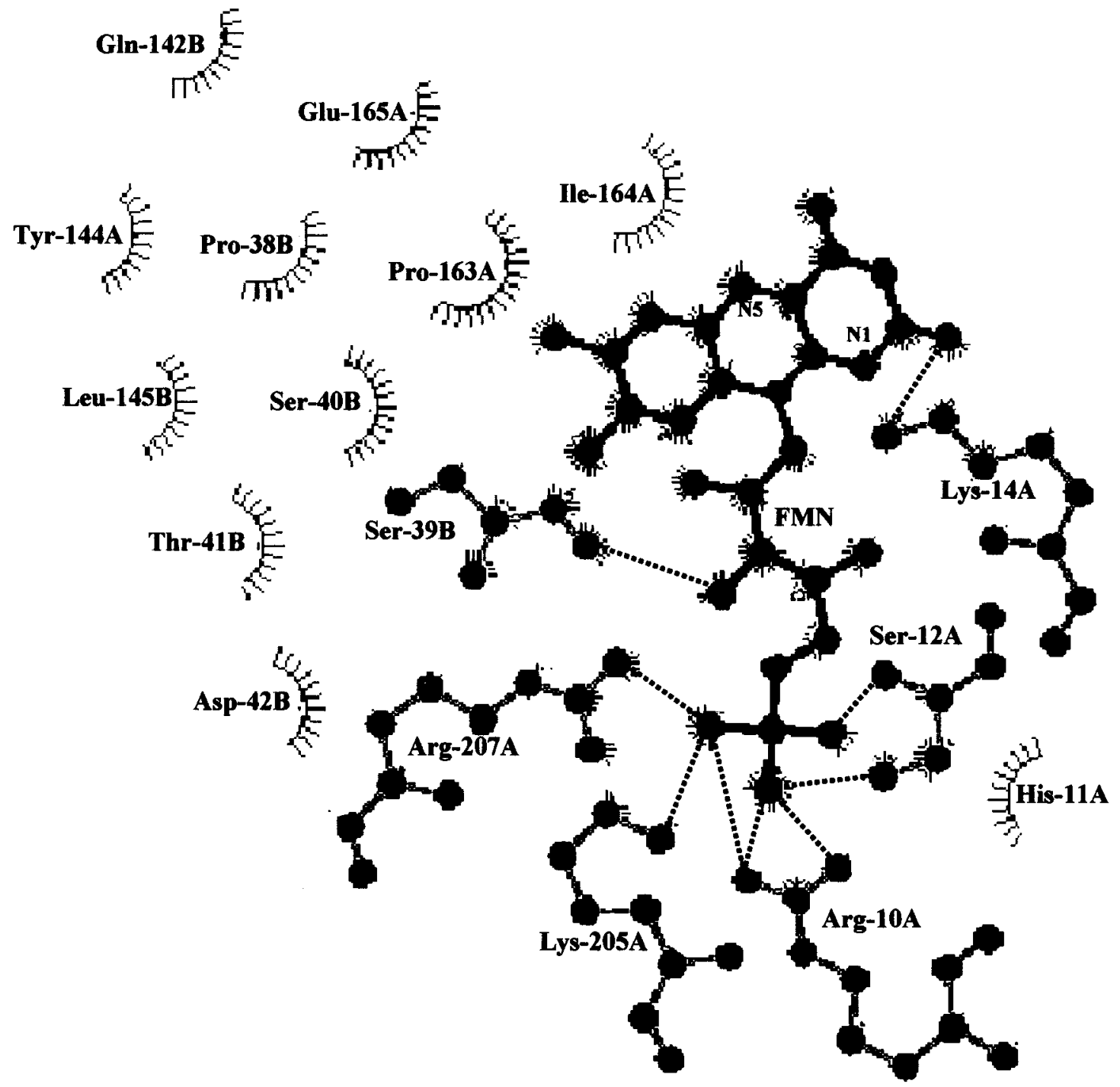

(a) FMN contacts in NfsB

Figure 4.6 LIGPLOT diagrams showing most of the structural contacts in NfsB with (a) FMN, and (b) NIO. Dotted lines $=$ hydrogen bonds. Arcs with spokes $=$ hydrophobic contacts. A and B refer to the two chains in the NfsB dimer. Refer to Figure 4.1 to see all of the structural contacts. 


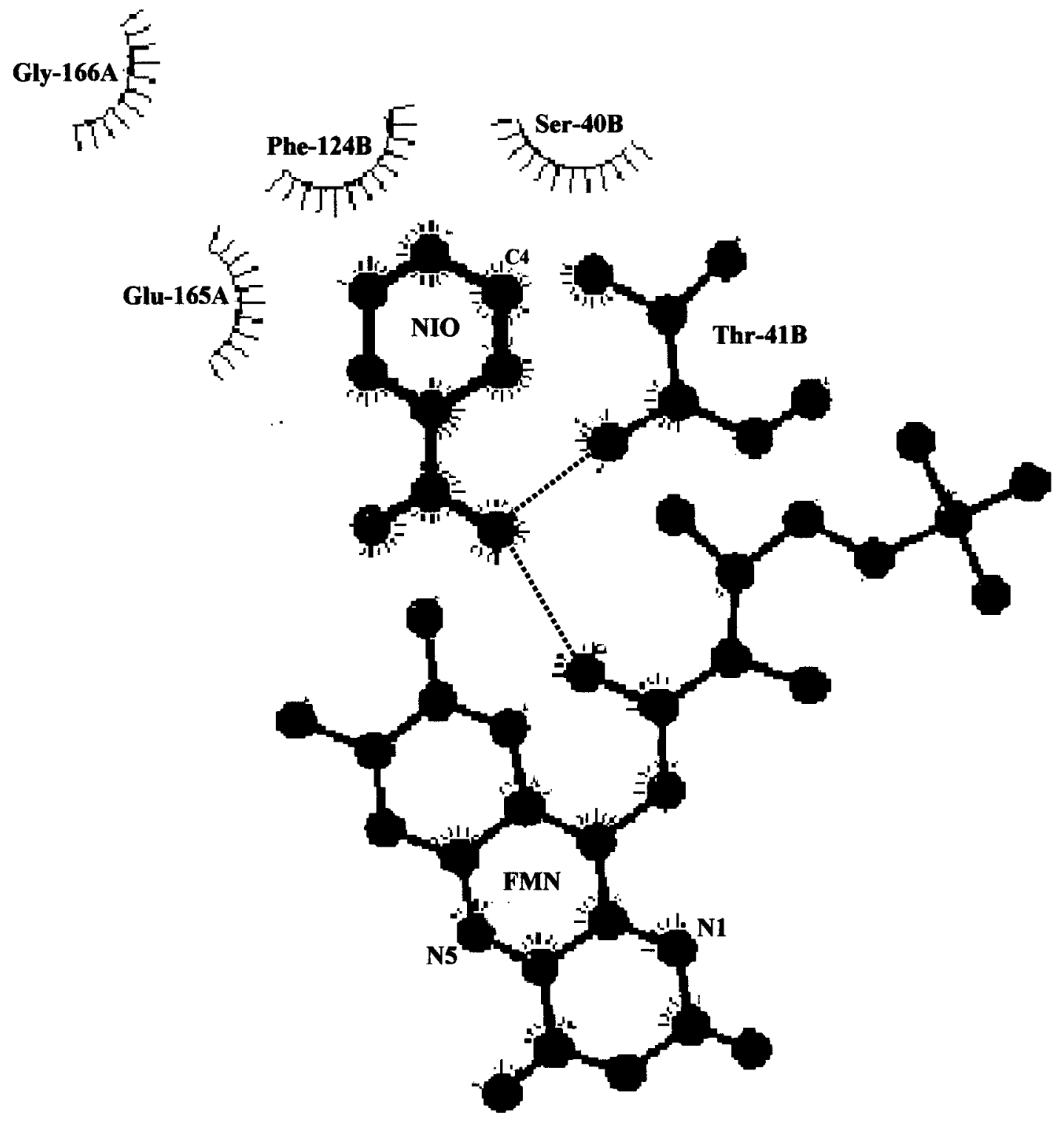

(b) NIO contacts in NfsB

Figure 4.6 (cont'd) 


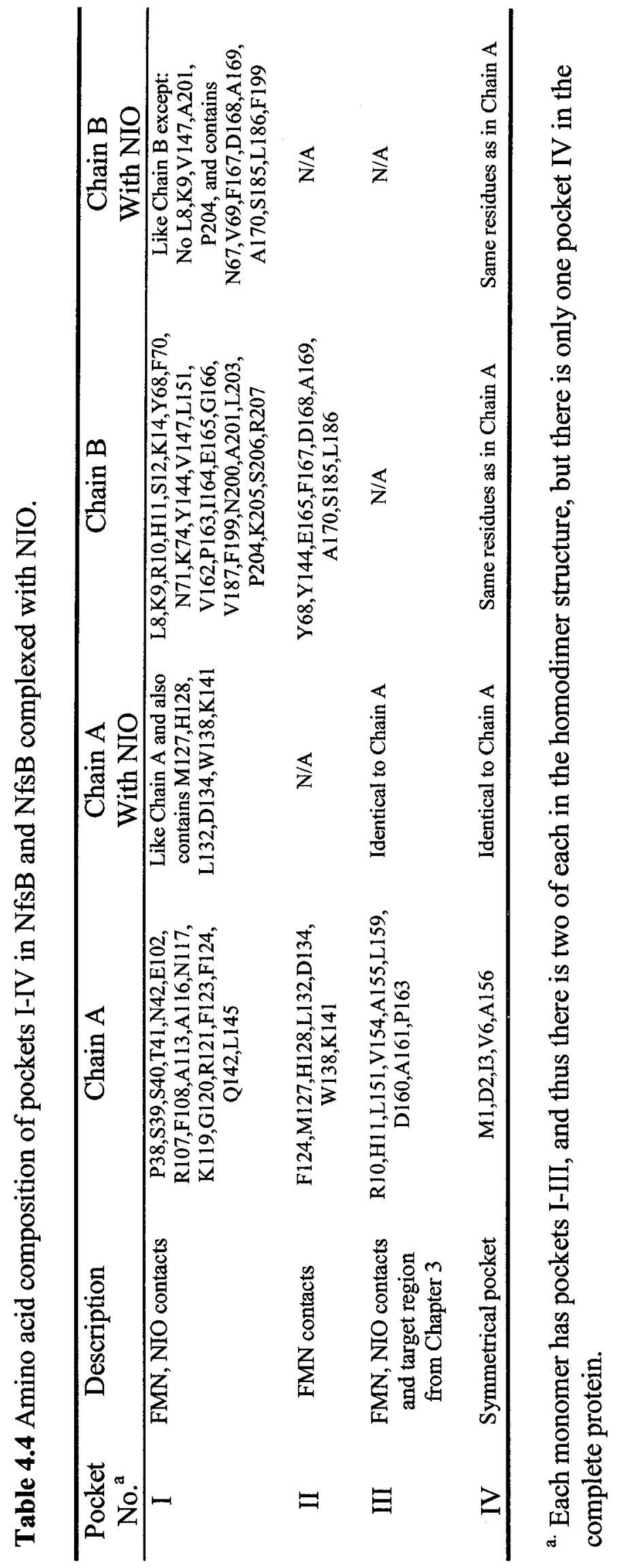


cofactor structures) and therefore a complete picture of $\mathrm{NAD}(\mathrm{P}) \mathrm{H}$ binding still does not exist.

A number of residues, notably the K117-F123 tract are located in the major pocket of NfsB but do not have contact with FMN or NIO. These residues are probably relevant for substrate specificity and/or the accommodation of cofactors as they are part of a less conserved helix that has residues associated with low B factors, indicating that this region is highly mobile; furthermore, these residues align with the large gap introduced in NfsA (see Section 4.3.3; Figure 4.1).

\subsubsection{Ancillary pockets}

Ligand binding pockets usually have a molecular volume within the range of $10^{2}$ $10^{3} \AA^{3}$, and one to two mouth openings (Liang et al., 1998). Pockets of this size adjacent to the primary active site pocket represent potential additional binding surface for ligands (Liang et al., 1998). Although smaller than the FMN binding pocket in NfsB, there are two pockets (II and III) in each monomer that are greater than $100 \AA^{3}$, plus there is one symmetrical pocket that is formed from equivalent residues in each monomer (IV) (Table 4.4, Figure 4.2). Pocket II is composed of residues from each monomer, and has two mouth openings, one of which is the shared small opening B of the main pocket (Figures 4.2\&4.5). Pocket III is completely formed within each monomer, has one mouth opening, is adjacent to the main pocket, on the opposite side from pocket II, and contains many of the residues that comprise the mutagenesis target region of Chapter 3. Pocket IV is sandwiched between pockets III of each monomer, and has two mouth openings. The 
size and location of these pockets suggests that they provide ligand contacts for $\mathrm{NfsB}$ and thus may be considered a part of the native active site.

In addition to size and proximity, conserved residues and cofactor induced conformational changes can provide signs that protein pockets may be considered part of the NfsB active site. Other regions of importance are implicated on the basis of comparison with the NADPH dependent enzymes (Section 4.3.3), and the structural alterations of the mutant proteins (Section 4.3.4).

\subsection{Conserved residues}

Most of the conserved residues revealed in Figure 4.1 have contact with FMN, or are located within the major pocket. However, there are two residues, Gln-44 and Gly192 in NfsB, that are conserved in both the NfsB- and NfsA-like enzymes, but do not have contact with FMN, and are not located in the major pocket. Gln-44 is within a tract of residues that are probably an important domain of NfsB-like proteins, as discussed below, and substitution of this residue results in the elimination of NR activity (Chapter 2). Gly-192 is located within a small cavity of NfsB that is not surface accessible, and the 8 residues with which it has contact (Phe-16, Lys-20, Leu-22, Ser-79, Leu-159, Asp160, Val-191 and His-193) are not located within the major pocket, nor do they have any contact with FMN or NIO. A low B-factor is associated with Gly-192 suggesting that its function is not to contribute flexibility, but rather to maintain structural integrity.

The stretch of residues from Gln-44 to Val-50 is highly conserved in NfsB homologues, as well as in the many putative homologues that have been identified from the burgeoning number of genome projects (for examples see Chapter 2, Table 2.5 and 
Figure 2.5). With the exception of Pro-45, all of these amino acids have contact with C-terminus excursion domain residues of the other monomer in the dimer structure. The residues in the excursion domain have relatively low B-factors, suggesting low mobility of this region. The low mobility associated with most of the conserved residues suggests a role in maintaining structural integrity in the enzymes rather than flexibility for the accommodation of substrates and cofactors.

\subsection{Conformational changes in NIO-bound NfsB}

An alignment of NfsB (1DS7) and NfsB bound with NIO (1ICR) reveals little change (RMSD of $0.4 \AA$ ) between the $\mathrm{C}^{\alpha}$ atoms of the two forms (Table 4.1). However, the RMSD of only the sidechains is $1.34 \AA$, and of all atoms is $0.98 \AA$. Upon examination of ICR it is apparent that the major pocket of ICR is actually a rough combination of pockets I and II in unbound NfsB (Table 4.4, Figure 4.5), which largely explains the much greater volume of the major pocket in ICR $\left(2520 \AA^{3}\right)$ compared to unbound NfsB (1302 $\AA^{3}$ ) (Table 4.3). An increase in the size of opening B (Figure 4.5) is also noticeable in ICR, which accounts for the CASTp program including both pockets as a single entity in this structure. In addition, there are numerous slight conformational changes in NIO-bound NfsB, some of which are mentioned in other sections, plus the altered side chain conformations of Phe-70 and Phe-124 seen in Figure 4.7 that appear to be particularly relevant.

\subsubsection{NfsA and Frp}

In the NfsA dimer structure, 60 pockets were identified, 30 per monomer, and 10 with volumes greater than $100 \AA^{3}$. Similarly, 10 pockets exceeding $100 \AA^{3}$ were 
a)
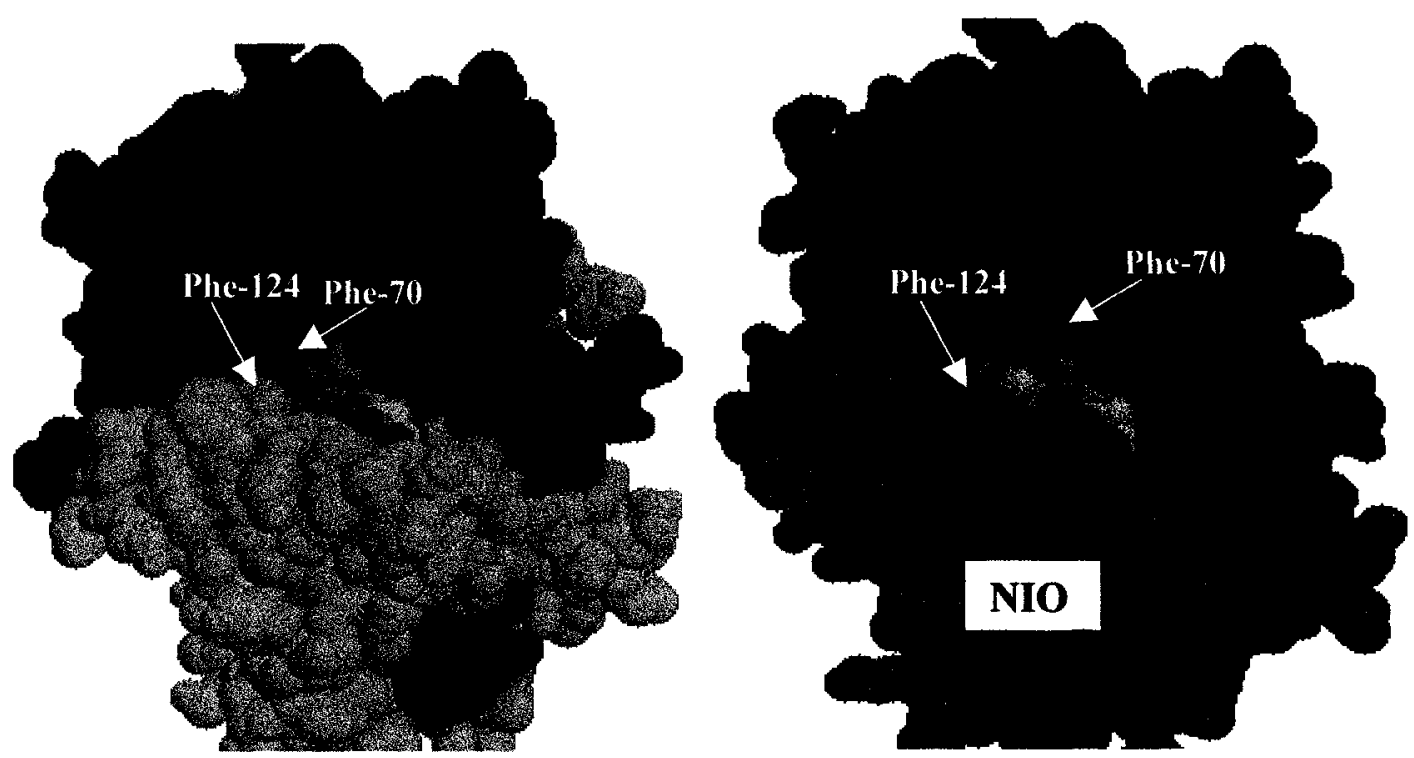

b)

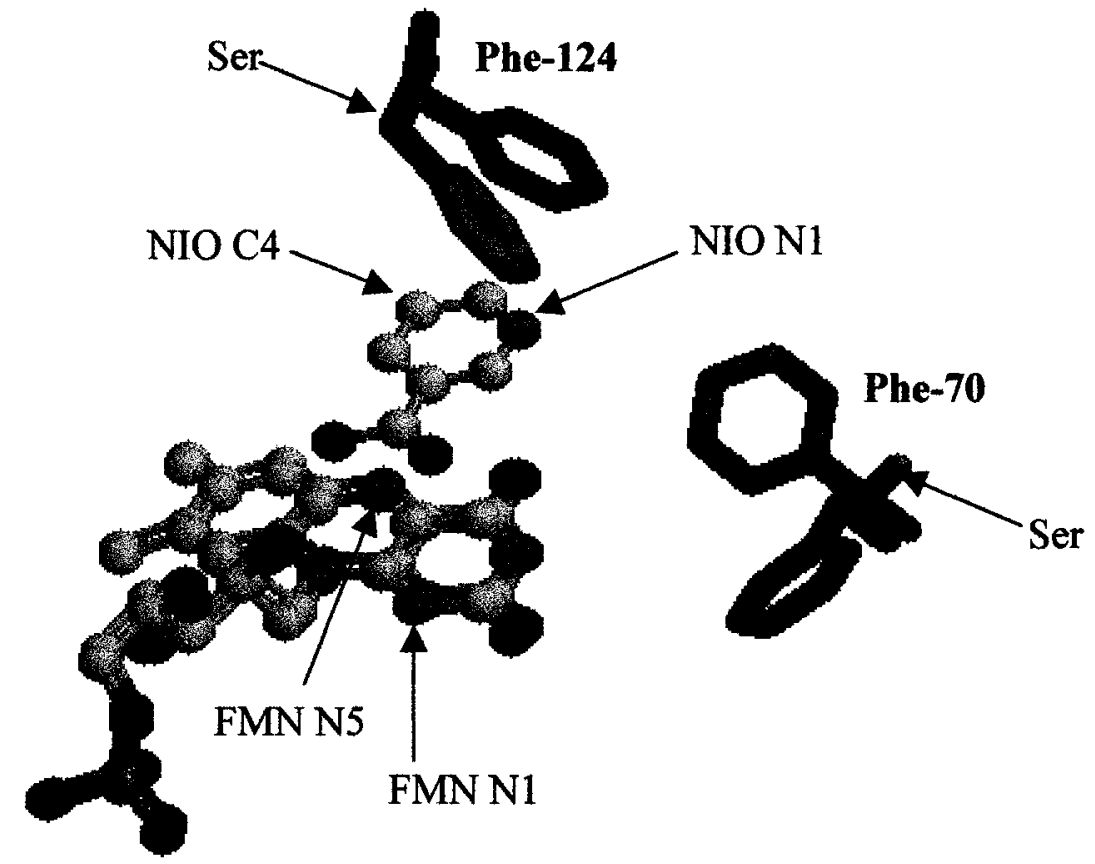

Figure 4.7 (a) Major pocket of NfsB and NIO-bound NfsB. Phe-70 and Phe-124 are indicated to illustrate the shift in sidechain position when $\mathrm{NfsB}$ is complexed with NIO. (b) Aligned FMN molecules, Phe-70 and Phe-124 in NfsB and NIO-bound NfsB. Green $=$ conformation in NfsB; Blue = conformation in NIO-bound NfsB. Yellow and red illustrate the F70S and F124S mutations, respectively. 
identified in Frp, which has 48 pockets in total, or 24 per monomer. Thus, each monomer of NfsA and Frp contained five pockets with volumes exceeding $100 \AA^{3}$.

\subsubsection{Major pocket}

The major pocket of NfsA is depicted in Figure 4.8 and the residues within this pocket are denoted for NfsA and Frp in bold italics on Figure 4.1. Notably, the volume of the major pocket is almost one-third less in the NfsA-like proteins (NfsA and Frp), approximately $1000 \AA^{3}$, as compared to the NfsB-like proteins (NfsB, NEC, VFR), about $1400 \AA^{3}$ (Table 4.3) despite the composition of the pocket being similar. The most notable difference in composition is the lack of any structure that aligns with $\alpha \mathrm{E}$ and $\alpha \mathrm{F}$ in NfsB (see Figures $4.1 \& 4.9$ ), including 11 residues that are located within the major pocket of NfsB.

All of the FMN contact residues are located within the major pocket of NfsA and Frp, and the majority of these align with the FMN contact residues in the NfsB-like proteins. The position of FMN is similar to the NfsB-like proteins, and is in the conserved butterfly conformation with the re face solvent accessible.

The major pocket of NfsA includes most of the $\mathrm{NAD}^{+}$contacts of Frp, many of which align with the NIO contacts of NfsB. The amino acids in the extended C-terminus domain, the excursion domain, of NfsA and Frp (see Figures 4.1 \& 4.9) can reasonably be expected to contribute to their exclusive use of NADPH. Two residues in this region of Frp, K219 and R225, do have contact with NAD ${ }^{+}$, and these align with a stretch of residues in NfsA that are located within its major pocket. 


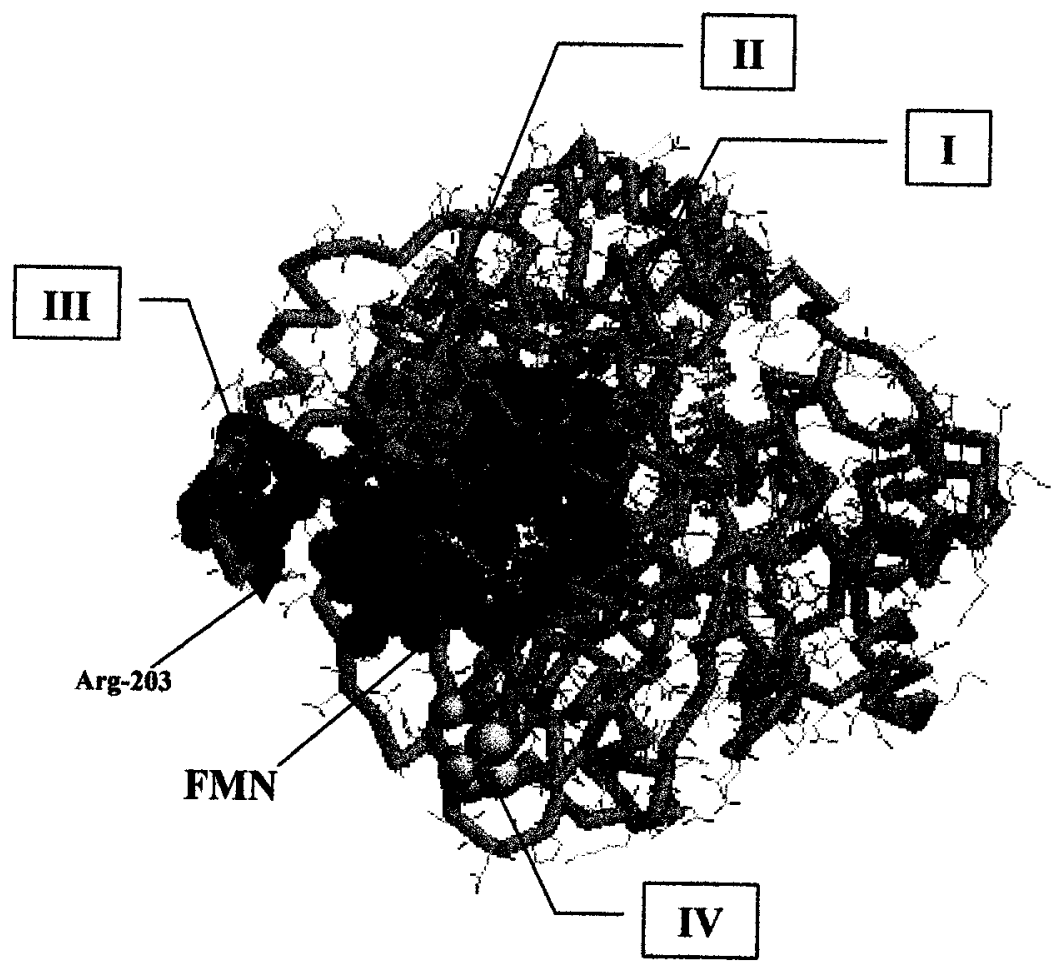

Figure 4.8 Structural pockets of NfsA. FMN is bound in the major pocket (red). The second largest pocket (II) is shown in grey. Refer to Table 4.3 for pocket volumes and mouth openings. 

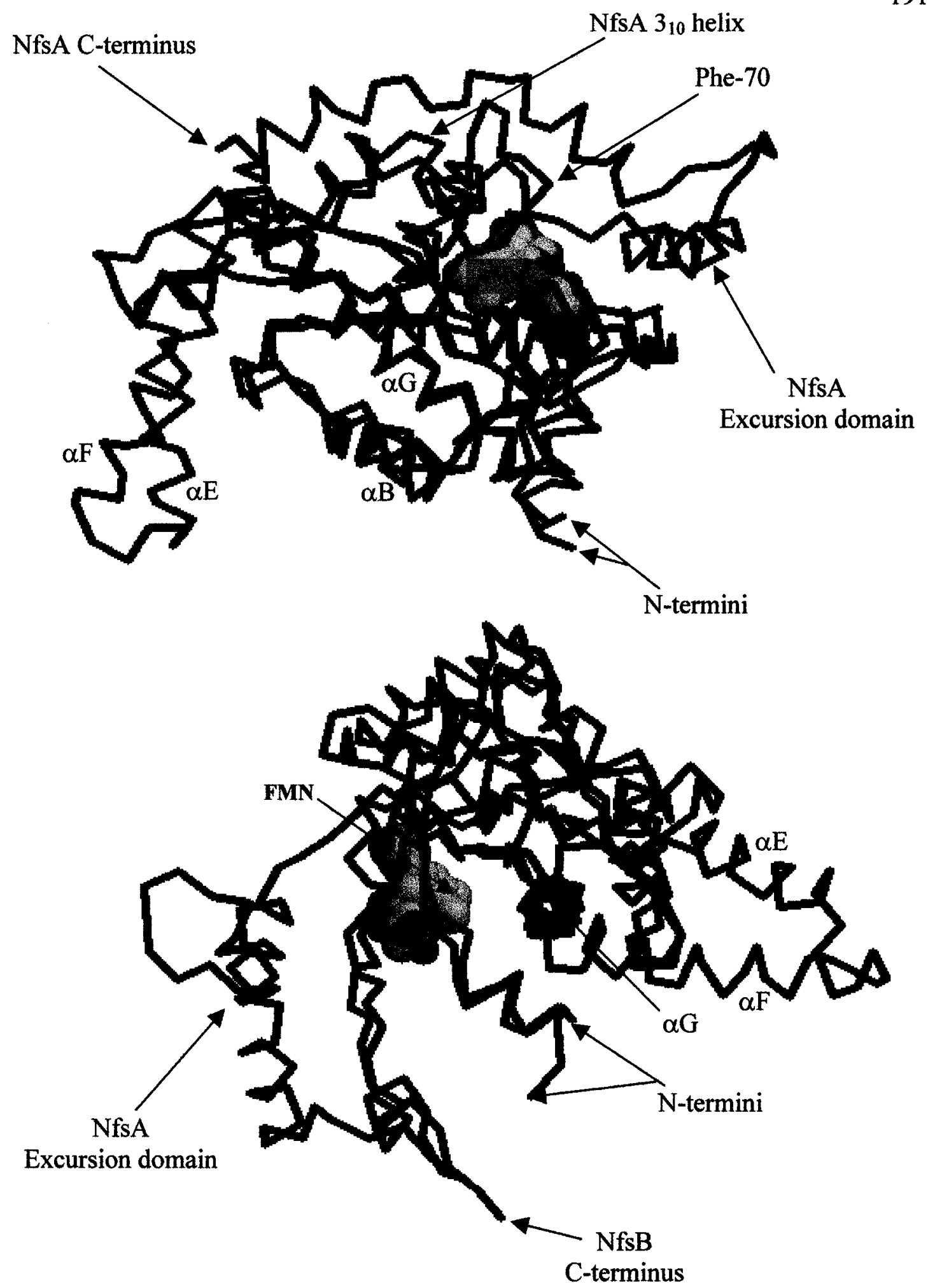

Figure 4.9 Two views of the aligned NfsA (red) and NfsB (blue) structures. Only one monomer is shown. Unless noted, the labels refer to NfsB. Refer to Figure 4.1 for sequence details. 


\subsubsection{Ancillary pockets}

In Frp, a few residues have contact with $\mathrm{NAD}^{+}$but are not within the major pocket. The associated residues in NfsA are mostly located within two pockets, labeled III and IV (Figure 4.8), that are near the mouth of the major pocket and are less than 100 $\AA^{3}$ in volume. Pocket III is within the excursion domain specific to NfsA and Frp. Pocket II is the second largest pocket in NfsA and is adjacent to the major pocket, but in this structure there are no solvent accessible mouth openings.

\subsubsection{Analysis of the mutant structures}

\subsubsection{Residues that influence $\mathrm{NAD}(\mathrm{P}) \mathrm{H}$ use}

In Chapter 3, the stretch of amino acids from Gly-153 to Leu-159 were analyzed for their contribution to the utilization of $\mathrm{NAD}(\mathrm{P}) \mathrm{H}$ in NfsB. The substitutions introduced at Val-154, Ala-155 and Leu-159, which are located within pocket II (Table 4.4), resulted in the elimination of NR activity. Pocket II also includes 3 amino acids, Arg-10, His-11 and Pro-163, that have contact with FMN.

Two mutants described in Chapter 3, G153S and G158S, retained some NR activity, with G153S exhibiting less NR activity than G158S. Although neither of these amino acids resides within a discernible pocket of wild-type $\mathrm{NfsB}$, it should be noted that in the mutant structure of G158S, the introduced Ser residue of one monomer is located within pocket IV along with another extra residue, Ala-155, from the same monomer. Gly-153 has contact with Leu-33, Phe-149, Leu-152, Val-154, Ala-156 and Leu-157 in NfsB. It may be that the lower NR activity of G153S relative to G158S is attributable to the contact of Gly-153 with Leu-33, a residue that has been implicated with cofactor 
utilization in the NfsB-like homologue Salmonella typhimurium CNR (Watanabe et al., 1998), and that results in the elimination of NR activity upon mutation in NfsB (Chapter 2).

The remaining amino acid of this mutagenesis target region, Leu-157, is not associated with a pocket in the native FMN oxidized structure of NfsB (Parkinson et al., 2000), but is in the structure resolved with bound NIO (ICR) (refer to Section 4.3.3.2.4). Furthermore, this residue also has contact with Leu-33.

\subsubsection{Residues that affect NR activity}

In Chapter 2, a number of missense mutations in $\mathrm{NfsB}$ resulted in a loss of NR activity (Table 2.2). Some of these mutations have been revealed in the preceding sections to affect residues that are highly conserved, have a putative role in cofactor utilization, and/or have contact with FMN. However, those reasons cannot be used to explain the influence of seven residues that were mutated: Leu-33, Leu-34, Ser-37, Val83, Asp-105, Gly-148 and Tyr-183. The effect on NR activity possibly is attributable to the following structural contacts. Leu-33, Ser-37, Val-83 and Gly-148 share pockets with FMN contact residues. As mentioned in Section 4.3.4.1, Leu-33 also has contact with Gly-153 and Gly-157, both of which are implicated in NAD(P)H utilization (Chapter 3), and is probably involved in cofactor utilization by CNR, the NfsB homologue of $S$. typhimurium (Watanabe et al., 1998). Asp-105 is situated between the flexible $\alpha \mathrm{E}$ and $\alpha \mathrm{F}$ helices that are specific to the NfsB-like proteins (Figure 4.9), and although this residue is not located within a pocket in native $\mathrm{NfsB}$, it does share a pocket with the conserved FMN contact residues Lys-205 and Arg-207 in NIO-bound NfsB. Likewise, 
Leu-34 and Tyr-183 are in different environments in NIO-bound NfsB, as compared to the unbound structure, and they share pockets with residues of the excursion domain, including the conserved region between Gln-44 and Val-50. 


\subsection{Discussion}

\subsubsection{Characterization of the oxidized NfsB active site}

NfsB contains two bound FMN molecules, one per monomer, and each is the foundation of an independently functioning active site (Parkinson et al., 2000). The FMN molecule is necessarily the reaction centre as it receives a hydride from the donating cofactor, NADH or NADPH, which it then uses to reduce the nitro-containing substrate in a ping-pong $\mathrm{Bi}-\mathrm{Bi}$ reaction mechanism (see Chapter 3, Figure 3.17). A ping-pong mechanism usually occurs in an enzyme with one primary ligand-binding site such that two ligands cannot bind at the same time, obliging the donor to leave before the acceptor can bind. In most enzymes the primary ligand-binding site is the largest surface accessible pocket of the protein (DesJarlais et al., 1988; Laskowski et al., 1996; Liang et al., 1998). In $\mathrm{NfsB}$, the largest pocket (approximately $1300 \AA^{3}$ ) is at the interface of the two monomers, and FMN is bound at the narrow end of the triangular cleft that they form (Figure 4.4).

The oxidized, bound FMN molecule is in a "butterfly" conformation with the re face exposed (Loverling et al., 2001; Parkinson et al., 2000). A butterfly conformation describes the bending of the two end rings of the isoalloxazine in the same direction, compared to the planar form of the free isoalloxazine ring system (Edmondson and Ghisla, 1999; Haynes et al., 2002). The FMN molecule is firmly bound in NfsB and its homologues with numerous protein contacts (Haynes et al., 2002; Koike et al., 1998; Loverling et al., 2001; Parkinson et al., 2000), and the protein interactions with FMN are barely disturbed upon binding of other ligands in NfsB (Loverling et al., 2001) or NEC 
(Haynes et al., 2002). The overall bend of oxidized FMN in NfsB is approximately $15^{\circ}$ (Loverling et al., 2001), and $16^{\circ}$ in E. cloacae NEC (Haynes et al., 2002), both in the direction of the protein occluded si face. This is a large angle, approximately only $10 \%$ of reported structures have similar or more extreme bends (Lennon et al., 1999). Haynes and coworkers suggest that the energetically unfavourable bend of FMN in the NR enzymes may be a tradeoff for an increase in the two-electron reduction potential of the oxidized flavin. The oxygen-insensitive NR enzymes are unusual in that there is no known one-electron reduced, or semiquinone, state (Mason and Holtzman, 1975). Semiquinone FMN is planar, similar to free oxidized flavin, and thus the obligatory twoelectron reduction of the Type I enzymes may be imposed by the severe bend in the bound flavin as it would be unable to stabilize the semiquinone form. Consequently, nitro compounds would be fully reduced rather than form oxygen radical species that cause cellular oxidative stress (Haynes et al., 2002). Evidence for NfsA (Liochev et al., 1999; Paterson et al., 2002) and NfsB (Liochev et al., 1999; Chapter 2, this thesis) having roles in the SoxRS response to oxidative stress indicates that this may be a key physiological role of the oxygen-insensitive NR enzymes in E. coli.

Based on the structure of native NfsB (Parkinson et al., 2000) in which access to the oxidized FMN molecule is restricted to the third ring of the isoalloxazine structure, the site of hydride transfer was originally predicted to be at FMN N1. However, as described in Section 4.4.2.1, the actual site of transfer is probably FMN N5, which suggests that upon binding of the cofactor there is a conformational change in NfsB. 


\subsubsection{Catalysis and the reduced form of $\mathrm{Nfs} B$}

\subsubsection{NAD(P)H}

In ICR, the structure of NIO-bound NfsB (Loverling et al., 2001), the planar nicotinamide ring stacks above the central FMN ring to allow hydride transfer (Figure 4.4). This stacking is also apparent for the planar inhibitors benzoate and acetate in $E$. cloacae NEC (Haynes et al., 2002). In ICR the C4 atom of the nicotinamide ring is $3.4 \AA$ from the FMN N5, which is close to the average distance of $3.5 \AA$ for hydride transfer at this atom (Fraaije and Mettevi, 2000), and places the pro- $R$ hydrogen facing FMN. Transfer to the FMN N5 atom is in accordance with a NMR study with the $V$. fischeri VFR homologue (Inouye and Nakamura, 1994), but it is at odds with the prediction derived from the oxidized form of $\mathrm{NfsB}$ that FMN N1 is the probable site of hydride transfer (Parkinson et al., 2000). This contrast between the bound and unbound structures of NfsB strongly suggests that there is a conformational change in NfsB after binding of the nicotinamide cofactor, such that hydride transfer occurs at the FMN N5 atom, as observed in VFR.

The structural alignment of oxidized NfsB with NIO-bound NfsB demonstrates that the conformational change in $\mathrm{NfsB}$ after binding is mainly due to alterations in the amino acid sidechains (RMSD 1.34 $\AA$ ) rather than in the C $\alpha$ backbone (RMSD $0.4 \AA$ ). However, a key structural change in NIO-bound NfsB, illustrated in Figure 4.5, is the incorporation of the two largest pockets (I and II) seen in unbound NfsB into one large pocket as a consequence of the widening of mouth opening B. Therefore, the ancillary pocket II, containing NIO contacts, can reasonably be expected to contain additional 
cofactor and substrate contacts that are made available to a substrate after cofactor induced conformational change. Furthermore, this larger pocket of NIO-bound NfsB has three mouth openings, compared to the two in unbound NfsB.

If the complete $\mathrm{NAD}(\mathrm{P}) \mathrm{H}$ cofactor is bound in $\mathrm{NfsB}$ in a similar orientation as $\mathrm{NIO}$, then the remainder of the molecule, including the adenine and ribose moieties, would be mostly solvent exposed. Consequently, there would be little interaction of the protein with the cofactor, which may explain the ability of $\mathrm{NfsB}$ to use the cofactors with comparable efficiency. In agreement with this is the indication that interaction of NEC with $\mathrm{NAD}(\mathrm{P}) \mathrm{H}$ is largely mediated by the amide moiety of the nicotinamide ring and that there is little protein-adenine interaction (Koder and Miller, 1998a). However, there are two indications of more extensive NfsB-cofactor interactions. First, the decreased NADPH specificity of the G158S mutant (discussed below) suggests that protein factors do play a role in NADPH utilization. And second, reduced ribotide cofactors tend to be active in NfsB, while reduced ribosides are not, which suggests that the 5 ' phosphate is required for cofactor recognition (Knox et al., 1995). The interesting exceptions to this cofactor specificity are 1-methylnicotinamide (Figure 4.3d), which does not have a ribose moiety, and reduced nicotinic acid riboside (Figure 4.3e), which does not have a 5'phosphate. However, both of these analogues exhibit decreased specificity for NfsB, and have $\mathrm{Km}$ values exceeding 5-10 times those obtained for NADH or NADPH (Knox et al., 1995). It may be that the 5' phosphate moieties aid in the binding and orientation of the complete cofactor, but due to less steric hindrance the smaller analogues do not 
require large conformational shifts to accommodate binding. This binding may approximate that of competitive inhibitors such as dicoumarol, benzoate and acetate.

The most common protein element that is involved in the binding of NADH is a Rossman fold, or mononucleotide-binding domain, which is composed of two parallel $\beta$ strands linked by an $\alpha$-helix such that the loops at the carboxyl termini of the $\beta$-strands interact with the phosphate group of the nucleotide (Carugo and Argos, 1997a; Wierenga et al., 1985). However, this motif is not present in NfsB or its homologues. Another common interaction specific to $\mathrm{NADH}$ is the presence of an aspartate or glutamate residue that hydrogen bonds with the diol group of the ribose near the adenine, and is followed by an apolar residue that interacts with the adenine (Carugo and Argos, 1997a). In the NfsB-like enzymes, Asp-168 is conserved and is often followed by an apolar residue. These residues follow the highly conserved FMN contact region from Val-162 to Gly-166 in NfsB, which includes two residues, Glu-165 and Gly-166, that bind NIO; therefore, it may be that Asp-168 and Ala-169 are involved in binding of the complete nicotinamide cofactor in $\mathrm{NfsB}$, depending on the conformation that the $\mathrm{NAD}(\mathrm{P}) \mathrm{H}$ cofactor adopts (discussed below). The N1 atom of NIO interacts with Gly-166 of NfsB, which indicates that the cofactor is oriented towards the small opening B in NfsB and is largely solvent exposed, although it might still have numerous other protein contacts within pockets I and II of NfsB.

$\mathrm{NAD}(\mathrm{P}) \mathrm{H}$ can adopt a variety of conformations in a protein (Figure 4.10) (Bell et al., 1997; Bellamacina, 1996; Carugo and Argos, 1997a; Tanner et al., 1998), so the actual conformation cannot be predicted from the binding of NIO alone. 

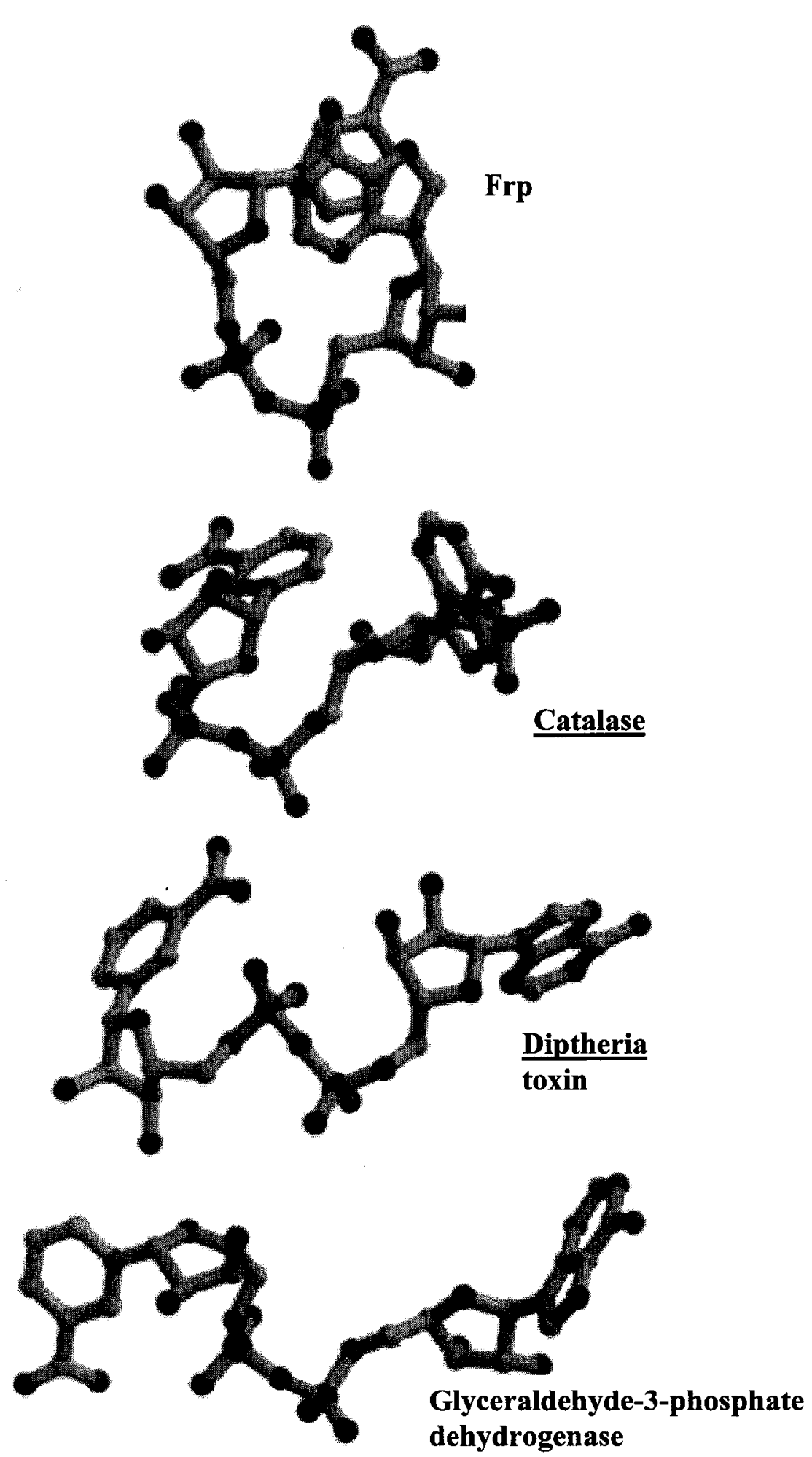

Figure 4.10 Some conformations of NAD. Adapted from Tanner et al., 1999. 
Protein interaction with the adenine component of NAD(P)H is minimal in NEC (Koder and Miller, 1998a), and thus this may be the case for NfsB too; nevertheless, as mentioned previously, there is some evidence for protein interaction with the 5, phosphate of the cofactor. The effect of the G158S mutation on NADPH utilization implicates protein-NADPH contact, even though Gly-158 probably does not interact with the cofactor directly as it is about $18 \AA$ from the FMN molecule. In the G158S mutant structure, Ser-158 and Ala-155 from one monomer are included in, and increase the volume of, pocket IV while having no apparent effect on the neighbouring pockets. This altered pocket or the disrupted contacts of Gly-158 could be responsible for the mutant enzyme's decreased specificity for NADPH. The most consistent feature of NADPH binding sites is the presence of an arginine that is able to face the adenine plane of NADPH; however, disruption of an arginine residue is not apparent in G158S. Gly-158 is associated with a low B-factor in the 3D structure of $\mathrm{NfsB}$, which suggests that this residue contributes stability rather than flexibility to the protein. The fact that Gly-158 is a long distance from the putative binding site of the nicotinamide cofactor does not negate its putative influence on cofactor specificity. In fact, residues as distant as $15 \AA$ from the bound $\mathrm{NAD}(\mathrm{P})^{+}$cofactor in $E$. coli glutathione reductase were shown to change the enzyme's cofactor specificity from $\mathrm{NADP}^{+}$to $\mathrm{NAD}^{+}$(Mittl et al., 1993,1994; Scrutton et al., 1990). Furthermore, one of these distant mutations resulted in the introduction of a residue that was hitherto not included in the known active site and yet helped alter the specificity for the 'wrong' cofactor (Scrutton et al., 1993). This is 
perhaps analogous to the introduction of Ala-155 and Ser-158 into pocket IV as seen for the G158S mutant of NfsB.

It is clear from the ICR structure and from the inhibitor bound structures of NEC (Haynes et al., 2002) that there is little room for any other molecule to bind when the cofactor is bound in the main pocket (Figure 4.7). This makes sense in the context of a ping-pong reaction mechanism, as it means that cofactor must leave before the substrate can bind.

\subsubsection{FMN}

A structure of the reduced form of the NfsB homologue NEC was recently resolved (Haynes et al., 2002). The coordinates of this structure are not yet released, but Haynes and coworkers report an increase in the isoalloxazine bend to $25^{\circ}$ from the $16^{\circ}$ of the oxidized form. At this severe angle, the structure is one of the largest bend angles reported for a protein bound FMN (Lennon et al., 1999), and is close to the approximated bend in free reduced flavin $\left(27-29^{\circ}\right)$ (Cavelier and Amzel, 2001). However, the protein contacts of reduced FMN in this structure are relatively undisturbed, as the bend is mainly about the N5-N10 axis (Haynes et al., 2002). Given the high degree of homology with NEC ( $88 \%$ protein sequence identity), it is reasonable to assume that reduced FMN in NfsB adopts a similar conformation. Indeed, despite obvious shifts in some of the amino acid sidechains of the major pocket in the NIO-bound structure of NfsB, FMN itself does not appear to change conformation (Figure 4.7b). 


\subsubsection{Substrate binding}

NfsB exhibits a broad substrate specificity for compounds of various sizes (McCalla et al., 1978; Zenno et al., 1996b), which suggests that the active site of this enzyme is quite flexible. In the structure of oxidized $\mathrm{NfsB}$, helix $\alpha \mathrm{F}$ is relatively unordered and contains residues with high B-factors, which implicates this region as being highly mobile. The helix becomes more ordered upon the binding of inhibitor (Parkinson et al., 2000) or NIO (Loverling et al., 2001). There is also a high degree of sequence variability between the NfsB-like enzymes within this region, especially within the stretch from Asn-117 to Phe-124 that corresponds to the large alignment gap in NfsA and Frp (Figures $4.1 \& 4.8$ ). The variability, mobility and presence of certain residues (discussed below) within the major pocket suggest that this region is involved in substrate specificity.

The stretch of residues from Pro-45 to Val-50 is highly conserved in the NfsBlike enzymes. In Figure 4.1, it can be seen that the QPW sequence is completely conserved, followed by a positively charged residue $(H / K / R)$ and three apolar residues. Although these residues are not within the major pocket, FMN and NIO contact residues within the major pocket directly precede this region in the primary sequence. However, the residues between Pro-45 and Val-50 are part of the domain that includes C-terminus residues from the other monomer of the dimer structure, which help form the mouth opening of the major pocket. The associated B-factors indicate that the Pro-45 to Val-50 region is not very mobile, a rigidity that is probably largely attributable to the conserved Pro-45 residue, and therefore it is unlikely that these residues shift to accommodate 
substrates. However, as Pro-45 to Val-50 are directly adjacent to the major pocket and $\mathrm{NIO}$ contact residues, it may be that the conserved QPWHFIV region of NfsB is responsible for the conformation of the NADH binding region and perhaps has contact with the cofactor itself.

Conformational changes that are obvious in NIO-bound NfsB include shifts in the aromatic sidechains of Phe-70 and Phe-124, which implicate their importance in substrate and/or cofactor binding. Notably, Phe-124 is within the flexible helix $\alpha F$ mentioned above. Examination of the 3D structures suggest that the shifts of Phe- 70 and Phe-124 in NIO-bound NfsB are crucial conformational changes (Figure 4.7) that allow access to either pocket I or II through opening B (Figure 4.5). There is also evidence in other studies for the participation of these residues in substrate binding. In the structure of VFR complexed with dicoumarol, Phe-124 shifts to accommodate the inhibitor (Koike et al., 1998), and Zenno et al. (1996b) demonstrated that the substitution of Phe-124 with a Ser residue alters the substrate specificity of NfsB such that it becomes an even more effective flavin reductase than its flavin reductase homologue VFR. Indeed, the major pocket of the F124S mutant is similar to NIO-bound NfsB in composition and volume (Table 4.3).

However, despite the structural similarity with NfsB, pocket analysis of VFR reveals a number of deviations that are probably attributable to the different substrate specificities of these enzymes. The equivalent pockets in VFR are larger than in NfsB, and the two largest pockets contain an extra mouth opening (Table 4.3). Flavins are large molecules and the ability to accept FMN as a substrate suggests that a large pocket 
volume is necessary. It can thus be reasoned that the F124S mutant of NfsB should have a greater volume in the binding site pocket(s) if the ability to catalyze flavin reduction is primarily a function of pocket volume rather than specific protein contacts in this enzyme. Indeed, the major pocket of $\mathrm{F} 124 \mathrm{~S}$ has about $50 \%$ greater volume than wild-type NfsB, and, like VFR, has three mouth openings instead of the two observed in wild-type NfsB. F124S is a temperature-sensitive mutant with respect to nitrofurazone reduction, which indicates that the mutation destabilizes the nitrofurazone-binding site. This destabilization appears to be due to altered protein contacts with nitrofurazone rather than a function of pocket volume, as a positively charged residue $(\mathrm{R} / \mathrm{H})$ does not decrease substrate reduction, while a negatively charged residue (D) drastically reduces the enzyme's ability to use nitrofurazone as a substrate (Zenno et al., 1996c).

Phe-70 is a solvent accessible residue in the stretch of residues that separates the mouth openings A and B of the major pocket in NfsB (Figures $4.5 \& 4.7$ ). Figure 4.1 reveals that this stretch of residues is also not well conserved, which may point toward a role in substrate specificity. Haynes et al. (2002) propose that this residue in NEC, along with Tyr-68 and Tyr-123, participates in substrate specificity by adjusting the side-chain conformation according to the substrate. In Chapter 3 of this thesis it was shown that $\mathrm{NfsB}$ exhibited an increased turnover number upon the substitution of Phe-70 with Ser. Cofactor specificity was not affected, so it could be that this residue is altered primarily to accommodate the subsequent substrate. If this change in conformation serves to reduce physical constraints on the incoming substrate, then the F70S mutant should reflect this as a greater pocket volume. Indeed, the major pocket of F70S does have a 
pocket volume that is approximately $200 \AA^{3}$ greater than wild-type NfsB, as well as having 3 mouth openings to the pocket akin to the NIO-bound structure of NfsB.

\subsubsection{Nfs $A$ and $N f s B: A$ revealing comparison}

The key biochemical distinction of NfsA compared to NfsB is its NADPH dependence. Otherwise, NfsA and NfsB share similar substrate specificity, although they reveal different efficiencies at reducing many substrates. NfsA has been designated as the major oxygen-insensitive nitroreductase component of $E$. coli due to its more rapid reduction of nitrofurazone: NfsA reduces nitrofurazone at a rate over five times that of NfsB (McCalla et al., 1978; Zenno et al., 1996a), even though there are some substrates, such as menadione, that are more efficiently reduced by NfsB (Doi et al., 1983; Zenno et al., 1996a). Other differences in substrate specificity between NfsA and NfsB include the lack of significant inhibition of NfsA by dicoumarol, and the inhibition of NfsA tartrazine azoreductase activity by exogenous FMN (Zenno et al., 1996a).

NfsA and NfsB share little sequence identity, about 16\%, but do have similar 3D structures (Figure 4.9). However, there are five fundamental structural differences between these enzymes that logically extend to their functional disparities, especially their different abilities to discriminate between NADPH and NADH. First, the major pocket of NfsA is about three-quarters of the volume of NfsB's major pocket (Table 4.3), which may contribute to the NADPH dependence of NfsA. The structure of $\mathrm{NAD}^{+}$bound Frp shows the $\mathrm{NAD}^{+}$molecule bound in a unique folded conformation, with the nicotinamide and adenine rings stacked in parallel (Figure 4.10), that is usually associated with NAD in solution (Tanner et al., 1999). In order to enter the narrow pocket of 
$\mathrm{NfsA} / F r p$, the cofactor probably enters the pocket 'backwards' with the 2' phosphate moiety stacked next to the flavin (Tanner et al., 1999). In NOX, VFR and NfsB a water molecule is bound at the same location as the phosphate in Frp. This folded conformation places the nicotinamide $\mathrm{C} 4$ atom $10 \AA$ from FMN N1, and as the required distance for hydride transfer is less than $4 \AA$ (Fraaije and Mettevi, 2000; Mittl et al., 1994; Tanner et al., 1999), the cofactor probably unfolds for catalysis. Recognition of the 2' phosphate may promote unfolding of the cofactor into the extended active form, and thus discriminate NADPH from NADH (Tanner et al., 1999).

The second key difference between NfsA and NfsB is the extended length of NfsA. The structural alignment shown in Figures 4.1 and 4.9 displays the 61 residue Cterminus extension of NfsA that comprises the excursion domain, which includes some residues (Thr-219 to Arg-225) that are part of the FMN binding pocket as well as the ancillary pocket III (Table 4.3, Figure 4.9). In the $\mathrm{NAD}^{+}$bound structure of Frp, three of the $13 \mathrm{NAD}^{+}$contacts are within this domain. In pocket III of NfsA, Arg-203 may contribute to the discrimination of NADPH, perhaps by recognition of the 2'-phosphate, as substitution with an Ala residue at this location results in a 33-fold increase in the $\mathrm{Km}$ value for NADPH (Kobori et al., 2001). However, $\mathrm{NADP}^{+}$is normally more flexible in binding to enzymes than $\mathrm{NAD}^{+}$(Carugo and Argos, 1997a), and it could be that the NADPH dependency of NfsA/Frp is due to a lack of specific recognition factors for $\mathrm{NADH}$, rather than a specific recognition factor for NADPH itself.

Third, both Arg-133 and Asn-134 are unique to NfsA/Frp as they are located within a $3_{10}$ helix that is not present in the NfsB-like enzymes (refer to Figures $4.1 \&$ 
4.9). In all of the NfsB-like structures a negatively charged Asp residue occupies the aligned Arg-133 position, followed by a small alignment gap. Asn-134 of Frp forms a hydrogen bond with the ribose near the adenine of $\mathrm{NAD}^{+}$, which, according to the LPC software, is a destabilizing contact. This contact could stabilize NADPH, which may help explain the specificity for this cofactor. In the NADP ${ }^{+}$-dependent 6phosphogluconate dehydrogenase, an Asn residue adjacent to an Arg has been shown to interact with the diol group of the ribose near the adenine of $\mathrm{NADP}^{+}$, an interaction that has not been observed with $\mathrm{NAD}^{+}$, and thus this region may represent a $\mathrm{NADP}(\mathrm{H})$ discriminating factor (Carugo and Argos, 1997a).

The fourth major difference between these enzymes is the alignment gap introduced in NfsA that overlaps helices $\alpha \mathrm{E}$ and $\alpha \mathrm{F}$ in NfsB (refer to Figures 4.1 \& 4.9). In particular, the flexible helix $\alpha \mathrm{F}$ of the NfsB-like enzymes has been implicated in the accommodation of substrates (see Section 4.4.2.3), while residues within and between these helices are involved in FMN and NIO binding (Figure 4.1). The alignment gap in $\mathrm{NfsA}$ and Frp also suggests that this region could play a role in the ability to accommodate both cofactors in NfsB. Indeed, four of the 12 NIO contacts in NfsB (Phe108, Ala-113, A116 and Phe-124) are within this region, and the absence of these contacts in NfsA may be key to the inability of this enzyme to utilize NADH. NOX has a ten-fold preference for NADH over NADPH as a cofactor (Hecht et al., 1995), and it may be that the smaller gap introduced within this region of NOX, spanning residues Phe-108 to Ala-116 of NfsB (see Figure 4.1), is a further indication that this region has a role in the accommodation of both cofactors. 
The fifth distinguishing feature of NfsB compared to NfsA is the NfsBspecific PW(HFIV) region that includes residues 45 to 50 in NfsB. This stretch of conserved residues is obvious in the NfsB homologues, but is lacking in the NfsA-like proteins. The positively charged $\mathrm{H} / \mathrm{K} / \mathrm{R}$ residue in the $\mathrm{NfsB}$-like enzymes is aligned with the polar Ser residue in NfsA, and it could be that this positive charge in NfsB acts to stabilize the negative diphosphate moiety of NADH. Such an interaction is often required for $\mathrm{NADH}$ binding, but has so far not been shown to be a requirement for NADPH binding.

It is interesting to note that, like the F124S mutation in NfsB, the substitution of Glu-99 with Gly alters the substrate specificity of NfsA such that it becomes an effective flavin reductase similar to its homologue Frp (Zenno et al., 1996a). The researchers that observed this mutant suggest that it is an increased pocket volume that leads to the altered substrate specificity; however, the major pocket only reveals a slight increase in volume, about $70 \AA^{3}$, and none of the other pockets significantly increase in volume (Table 4.3). It may be that this slight volume increase is sufficient for the altered specificity, or that the pocket volume in the FMN reduced conformation of NfsA is substantially different, but it could also be that other factors are responsible for the altered substrate specificity. The more drastic increase in pocket volume observed for the NfsB F124S mutant suggests that the mode of altered substrate specificity is different for NfsA.

The small volume of the major pocket in NfsA and Frp suggests that either a dramatic change in conformation takes place after cofactor binding, or the adjacent pockets of the enzymes have the space and contacts to accommodate bulky substrates. 
Pocket analysis of NfsA reveals an above average number of mouth openings (four) in the major pocket (Liang et al., 1998), which could be an indication that adjacent pockets contribute protein contacts for ligands. Unlike NfsB, a large increase in pocket volume is not observed upon binding of a cofactor analogue in $\operatorname{Frp}\left(\mathrm{NAD}^{+}\right)$, or in the NfsA flavin reductase mutant, E99G, that must accommodate large flavin molecules in its active site. It is possible that, like pocket IV in NfsB, other pockets affect catalysis despite not being directly adjacent to the major pocket, but it is difficult to assess these effects without mutant analysis or binding experiments. The second largest pocket of NfsA is adjacent to the major pocket but has no mouth openings. Perhaps, similar to pocket II of $\mathrm{NfsB}$, cofactor binding provokes a conformational change that results in this pocket being included in the active site. Two smaller pockets that are likely involved in the active site of NfsA are near the major pocket. Pocket IV contains some of the equivalent $\mathrm{NAD}^{+}$contacts in Frp, while pocket III is situated near the mouth opening and contains Arg-203, a residue that has been implicated in the use and/or binding of NADPH (Kobori et al., 2001). It is possible that most bound substrates are necessarily solvent exposed due to the small volume of the major pocket, which could help explain the broad substrate specificity of these enzymes, especially the ability to bind such large substrates.

\subsubsection{The implications for structure-based drug development}

\subsubsection{NfsB}

Pockets adjacent to the primary active site pocket present additional binding surface for ligands. Ligand contacts in ancillary pockets and cavities have been successfully predicted for some proteins using the CAST software, including elastase, 
FK506 and HIV-1 protease (Liang et al., 1998). Furthermore, the recruitment of nearby pockets that do not have contact with substrate can provide additional protein contacts for designed ligands (Mattos et al., 1994; Mattos and Ringe, 1996; Shuker et al., 1996). The prospect of ligand design in NfsB is important for the utilization of modified cofactors (Knox et al., 1995) and novel prodrugs (Denny, 2002) in targeted cancer therapy approaches (see Section 1.3.1.2), as well as for antimicrobial drug development. Prediction of additional binding sites in NfsB could aid in the development of highly specific substrates that would be less vulnerable to the development of resistance or activation by host enzyme activity. The incorporation of pocket II with the main active site pocket in NIO-bound NfsB suggests the importance of this region for enzyme function, while pocket III is a good candidate for recruitment into a rational redesign approach for drug development.

\subsubsection{NfsA}

Whereas NfsB is being actively explored as a promising candidate in A/GDEPT cancer therapy approaches, NfsA is not, which may simply be a consequence of the earlier characterization of $\mathrm{NfsB}$. However, there is some investigation into the use of NfsA in A/GDEPT strategies (Bennett and Lambert, manuscript in preparation) and genotoxicity assays (Carroll et al., 2002), and given its more efficient NR activity with some compounds of commercial and environmental importance, it is a promising candidate for such applications. Here, then, as in $\mathrm{NfsB}$, the ability to predict potential binding sites, and design specific ligands is a valuable asset for drug development. With four mouth openings in the major pocket of the oxidized state of NfsA, and a number of 
adjacent pockets of reasonable size, it appears that there are numerous candidate sites that could be exploited for drug development. For example, pocket II (Table 4.2), the second largest pocket in NfsA, is probably part of the active site, and is a promising candidate for potential ligand contacts. Smaller pockets that are adjacent to the main pocket (e.g. pockets III and IV) may also be involved in catalysis, and/or may be recruited for ligand design in NfsA. 


\section{CHAPTER FIVE: CONCLUSIONS AND SUGGESTIONS FOR FURTHER RESEARCH}

Oxygen-insensitive nitroreductases are becoming recognized as a widespread family of enzymes. Over 140 putative enzymes have been identified that span the three main domains of life: Bacteria, Eukaryota and Archaea. The NfsB protein of $E$. coli is involved in the bioactivation of nitro substituted compounds, and its activity is being exploited for a number of applications including genotoxicity assays and cancer therapy. However, the physiological role of $\mathrm{NfsB}$ and other nitroreductases remains a mystery. The work reported in this thesis contributes to the rapidly accumulating knowledge of $\mathrm{NfsB}$, and thus of the nitroreductase family, in three main areas: its role in the development of 5-nitrofuran resistance; its structure-function relationships; and its possible physiological role.

The investigations reported in this thesis give rise to a number of additional questions and emphasize the importance of several issues that preexisted this research. Here, the unifying and pertinent conclusions that arose from these studies are described along with directly related questions that would represent the most interesting and logical continuation of this work.

\subsection{Development of 5-nitrofuran resistance in $E$. coli}

The nitrofurazone reductase activity of a large number of nitrofuran resistant mutants was determined and the $n f s B$ gene was genetically characterized in order to test the hypothesis that the second step of $E$. coli resistance to furazolidone and nitrofurazone is due to the mutational inactivation of the $n f s B$ gene. Koziarz (2000) determined that the first step of resistance correlates with the mutational inactivation of the $n f s A$ gene in these mutants. 


\subsubsection{Conclusions}

1. 5-Nitrofuran resistance is acquired through a sequential mechanism that involves the obligatory mutational inactivation of $n f s A$ (first step mutant), followed by the inactivation of $n f s B$ (second step mutant).

2. Mutant cross resistance is observed with furazolidone and nitrofurazone. Mutants that display an inconsistent third step of resistance to furazolidone exhibit second step levels of resistance to nitrofurazone. This phenomenon, previously demonstrated with nitrofurantoin (Obaseiki-Ebor, 1984), does not appear to be a factor of decreased permeability for furazolidone.

3. The $n f s B$ gene contains integration hotspots for the IS elements IS 1 , IS 5 and perhaps IS2.

\subsubsection{Suggestions for further research}

1. The third step of resistance that is associated with furazolidone and nitrofurantoin but not with nitrofurazone (Section 2.3.1) warrants further investigation. One possibility is that other nitroreductase activities are involved in the metabolism of these compounds. There is some biochemical evidence for a third oxygeninsensitive nitroreductase component in $E$. coli (Bryant et al., 1981), and double $n f_{s} A / n f s B$ mutants do retain a low level of nitroreductase activity (Chapter 2, Table 2.2). It could be that one or both of the two $E$. coli sequences identified by the Pfam database as putative nitroreductase genes represent this minor nitroreductase component (see Chapter 1, Section 1.2). The sequences of these genes in the third step mutants, as well as in the second step mutants for which no 
mutation in $n f_{s} B$ was found (Section 2.3.2; Table 2.2), could be compared with the parental strains. Any observed variation between the mutant and parental strains would make the cloning of these new genes, and characterization of their products, even more compelling.

2. The unanticipated number of IS element integrations in $n f s B$ opens an appealing avenue of research. Interestingly, a large number of IS elements were also observed in the $n f s A$ gene of first step mutants (Koziarz, 2000). The putative IS element hotspots in $n f_{S} B$ (and $n f_{S A}$ ) may implicate a horizontal gene transfer mechanism by which the nitroreductase family could have evolved to what now appears to be a widespread family of enzymes. The notion that nitroreductase genes were horizontally transferred by means of transposable elements (TEs) is plausible, especially given the putative role of nitroreductases in the bacterial response to environmental stress (Section 5.3). In addition, TEs are often activated under conditions of environmental stress (Chao et al., 1983; Kidwell and Lisch, 1997; McClintock, 1984), including exposure to DNA damaging agents (Eichenbaum and Livneh, 1998). Thus, two related questions arise from this phenomenon that would be interesting to investigate:

i. Do 5-nitrofurans, which are DNA damaging agents, stimulate TE activity in E. coli?

ii. Does the $n f s B$ gene contain hotspots for IS element integration, or are the large number of IS element integrations observed in this study simply a consequence of furazolidone stimulated TE activity? 
To test the hypothesis that 5 -nitrofurans stimulate TE activity it would be necessary to compare IS element activity of untreated and nitrofuran-treated cells, preferably within the context of the whole genome. A comparison of whole genome IS element activity may perhaps be achieved by the technique of bacterial comparative genomic hybridization (BCGH) (Malloff et al., 2001). Essentially, this technique compares the genome of two related bacterial strains by digesting and combining their genomic DNA, resolving the DNA on a 2D gel, transferring it to a membrane, and probing with a total genomic DNA probe from each strain. For the purposes of this study, the genomic variance associated with particular IS elements could be identified by probing with specific IS element probes. Thus, genomic variance as a consequence of nitrofuran treatment can be compared between strains, particularly that which is attributable to the activity of specific IS elements.

The hypothesis that $n f s B$ contains hotspots for IS elements can be tested by introducing $n f s B$ on a plasmid, as a TE trap, into a $\triangle n f s A \Delta n f s B$ host. Selection of nitrofuran resistant mutants, followed by Southern blot analysis of the extracted plasmid DNA would facilitate determination of mutant frequencies and analysis of the number and type of IS elements.

\subsection{Structure and function of $\mathrm{NfsB}$}

In this study, the structure and function of NfsB was assessed in two ways: first, by mutagenesis and kinetic analysis; and second, by computer analysis of the $3 \mathrm{D}$ crystal structure of NfsB and its homologues. Ideally, these studies would have been approached 
in the reverse order, but the mutagenesis study was completed prior to the availability of the $3 \mathrm{D}$ structure. In addition to these two studies, information on the structure and function of $\mathrm{NfsB}$ was obtained through the investigation of 5-nitrofuran resistance development which identified residues whose mutation eliminated nitroreductase activity.

\subsubsection{Conclusions}

1. A number of features of NfsB that had been determined, or suggested, in other studies were confirmed here:

i. The $n f s B$ gene has a promoter within $148 \mathrm{bp}$ of the initiation codon (Chapter 2).

ii. The NfsB protein is about $24 \mathrm{kD}$ and appears as approximately $27 \mathrm{kD}$ on a denaturing SDS-PAGE gel (Chapter 3); it can use both NADH and NADPH as a source of reducing equivalents, with a slight preference for NADH (Chapter 3); and reduces nitrofurazone by means of a mechanism that is consistent with a ping-pong $\mathrm{Bi} \mathrm{Bi}$ reaction type (Chapter 3).

2. Unique to this study is the identification of numerous residues that are essential for nitroreductase activity. These are revealed by the single missense mutations that confer a nitrofuran resistant phenotype (Chapter 2), and by those introduced through mutagenesis that resulted in null or altered nitroreductase activity (Chapter 3). Many of these residues are involved in FMN binding, but several others appear to be associated with $\mathrm{NAD}(\mathrm{P}) \mathrm{H}$ and/or substrate binding.

i. The region from Pro-45 to Val-50 is probably involved in substrate specificity and is also a key characteristic of the NfsB-like proteins that 
distinguishes them from the NfsA-like proteins (Chapter 4). The $H$.

pylori $\mathrm{RdxA}$ protein, which is more similar to $\mathrm{NfsB}$ than $\mathrm{Nfs} A$ in terms of sequence and activity, contains this conserved region, but uses NADPH exclusively, so it is unlikely that this region specifies NADH utilization.

ii. Gly-158 appears to be significant for the utilization of NADPH, but not NADH (Chapter 3).

iii. Phe-70 does not affect $\mathrm{NAD}(\mathrm{P}) \mathrm{H}$ utilization, but does have an effect on the catalytic efficiency of $\mathrm{NfsB}$, most likely by influencing the transition energy of catalysis (Chapter 3). Positioned between the two main pockets of NfsB, Phe-70 may be key for shifting conformation to accommodate the binding of bulky substrates (Chapter 4).

iv. The region from approximately Phe-108 to Asp-135 is largely comprised of a long, flexible $\alpha$-helix $(\alpha \mathrm{F})$ and is not well conserved. This region is probably a major contributor to the ability of $\mathrm{NfsB}$ and its homologues to accommodate the wide range of bulky substrates characteristic of these proteins (Chapter 4).

3. NfsB has seven key surface accessible pockets that can reasonably be expected to be involved in catalysis for reasons of size, proximity to FMN, and/or inclusion of critical residues (i.e. conserved or indicated by mutation analysis) (Chapter 4). There are three similar pockets in each monomer, for a total of six pockets per dimer, and one pocket that is symmetrically comprised of residues from each 
monomer. These seven pockets represent important structural elements to be considered in the design of drugs and ligands for NfsB.

4. There are five main features that distinguish the NfsB-like proteins from the NfsA-like members of the nitroreductase family (Chapter 4, Section 4.4.4):

i. The main active site pocket of NfsB is about one third larger than NfsA.

ii. NfsA is larger than NfsB by 23 amino acids and contains an extended Cterminus domain of about 60 residues.

iii. NfsA contains a $3_{10}$ helix that is not present in NfsB.

iv. NfsA does not have the flexible $\alpha$ helix $(\alpha \mathrm{F})$ mentioned in 5.2.1, 2(iv) above that is implicated in the substrate binding of $\mathrm{NfsB}$.

v. As mentioned in 5.2.1, 2(i) above, the NfsA-like proteins lack the highly conserved Pro-45 to Val-50 region of the NfsB-like proteins.

\subsubsection{Suggestions for further research}

1. The kinetic analysis of this investigation confirms that $\mathrm{NfsB}$ reduces nitrofurazone according to a ping-pong reaction mechanism. However, it is not clear that this is a $\mathrm{Bi} \mathrm{Bi}$ mechanism, as the data is also consistent with a Hexa Uni reaction mechanism. To determine the specific reaction type of $\mathrm{NfsB}$, isotope exchange experiments and kinetic analysis of the reverse reactions of $\mathrm{NfsB}$ could be performed. Identification of the actual reaction mechanism of NfsB would be facilitated by the determination of the reaction intermediates of the reaction, particularly whether the first released intermediate is reduced by 2 or 4 electrons. 
2. The mutations analyzed in this study suggest that the mutated residues influence nitroreductase activity. However, it is possible that at least some of the mutations were simply too drastic and that substitution with similar residues may yield information regarding the influence of the residue(s) on catalysis. For example, some substitutions of the amino acids that were mutated in Chapters 2 and 3 could be made with more evolutionarily accepted residues (for example with those listed in Table 3.8).

3. Some combinations of mutations could be informative. For example, Gly$158 \rightarrow$ Ser combined with Phe- $70 \rightarrow$ Ser produces an enzyme with high activity along with increased specificity for $\mathrm{NADH}$.

\subsection{The Physiological Role of $n f_{s} B$}

Mutants lacking a functional NfsB enzyme are easily cultured under normal conditions, which indicates that this enzyme is not an essential constituent of $E$. coli, at least under laboratory conditions. The only known activity of $\mathrm{NfsB}$ is the reductive metabolism of nitro containing compounds. These features are both consistent with the view that NfsB has a role in the defense of the cell against nitro substituted xenobiotic compounds.

The high frequency of IS element insertions observed in nitrofuran resistant mutants also concurs with this notion, as TEs are often associated with (groups of) genes that are involved in environmental stress responses. For example, the rapid transmission of a variety of genes associated with the catabolism of aromatic environmental pollutants is often facilitated by TEs (for reviews see Tan, 1999; Wyndham et al., 1994). A few 
more examples of environmental response genes that are likely transmitted by transposons include mercury resistance genes (Osborn et al., 1997), catalases (Klotz et al., 1997) and the histidine kinases of Archaea (Kim and Forst, 2001).

Recent studies have indicated that NfsA is part of the SoxRS response to oxidative stress (Liochev et al., 1999; Paterson et al., 2002), and that both NfsA and NfsB are members of the associated MarRAB regulon (Barbosa and Levy, 2000). To test for the participation of $\mathrm{NfsB}$ in these regulons, induction with paraquat (SoxR specific) and salicyclate (MarA specific) was demonstrated (Chapter 2), suggesting that NfsB is part of the SoxRS/MarRAB regulated global response to environmental stress.

\subsubsection{Suggestions for further research}

1. The regulatory regions of $n f s B$ should be characterized. While no marbox consensus sequence has been identified to date, DNA footprinting and gel shift assays using the regulatory elements of the operons (i.e. SoxR and MarA) would reveal binding site(s) for these proteins.

2. The concept of NfsB as a member of a global response to environmental stress can be expanded and tests for other environmental factors such as a pollutants, UV light and starvation could help to reveal the role of NfsB in the defense mechanisms of $E$. coli.

3. Identification of the cell cycle expression profile of $\mathrm{NfsB}$ may also yield clues as to the physiological role of the enzyme. For instance, in Caulobacter crescentus enzymes involved in the oxidative response are mainly expressed in G1, which is suggested to be a way that the organism protects against DNA damage during 
replication (Grünenfelder et al., 2001). This would be most easily facilitated by microarray analysis for gene expression such that a global representation of the cell's entire gene expression profile would be observed. 
APPENDIX I:

The one and three letter codes of the 20 amino acids found in proteins.

\begin{tabular}{lcc}
\hline Amino acid & Three letter code & One letter code \\
\hline Alanine & Ala & A \\
Arginine & Arg & R \\
Asparagine & Asn & N \\
Aspartic acid & Asp & D \\
Cysteine & Cys & C \\
Glutamic acid & Glu & E \\
Glutamine & Gln & Q \\
Glycine & Gly & G \\
Histidine & His & H \\
Isoleucine & Ile & I \\
Leucine & Leu & L \\
Lysine & Lys & K \\
Methionine & Met & M \\
Phenylalanine & Phe & F \\
Proline & Pro & P \\
Serine & Ser & S \\
Threonine & Thr & T \\
Tryptophan & Trp & W \\
Tyrosine & Tyr & Y \\
Valine & Val & V \\
Unknown/unspecified & Xaa & X \\
\hline & &
\end{tabular}




\section{LITERATURE CITED}

Aghi, M., F. Hochberg, and X. Breakefield. 2000. Prodrug activation enzymes in cancer gene therapy. J Gene Med 2:148-164.

Allison, R.D., and D.L. Purich. 1979. Practical considerations in the design of initial velocity enzyme rate assays. Methods Enzymol. 63:3-22.

Altschul, S., W. Gish, W. Miller, E. Meyers, and D.J. Lipman. 1990. Basic local alignment search tool. J Mol Biol. 219:555-585.

Altschul, S.F., T. Madden, A. Schäffer, J. Zhang, Z. Zhang, W. Miller, and D. Lipman. 1997. Gapped BLAST and PSI-BLAST: a new generation of protein database search programs. Nucleic Acids Res. 25:3389-3402.

Ames, B.N. 1979. Identifying environmental chemicals causing mutations and cancer. Science 204:587-593.

Amzel, L.M. 1998. Structure-based drug design. Curr Opin Biotech. 9:366-369.

Anderson, P., L. Cole, D. McKay, and B. Entsch. 2002. A flavoprotein encoded in Selenomonas muminantium is characterized after expression in Escherichia coli. Protein Expr Purif. 24:429-438.

Anlezark, G.M, R.G. Melton, R.F. Sherwood, B. Coles, F. Friedlos, and R.J. Knox. 1992. The bioactivation of 5-(aziridin-1-yl)-2,4-dinitrobenzamide (CB1954). 1. Purification and properties of nitroreductase enzyme from $E$. coli, a potential enzyme for antibodydirected enzyme prodrug therapy (ADEPT). Biochem Pharm. 44:2289.

Anlezark, G.M, R.G. Melton, R.F. Sherwood, W.R. Wilson, W.A. Denny, B.D. Palmer, R.J. Knox, F. Friedlos, and A. Williams. 1995. Bioactivation of dinitrobenzamide mustards by an $E$. coli B nitroreductase. Biochem Pharm. 50:609-618.

Anlezark, G., T. Vaughan, E. Fashola-Stone, N. Michael, H. Murdoch, M. Sims, S. Stubbs, S. Wigley, and N. Minton. 2002. Bacillus amyloliquefaciens orthologue of Bacillus subtilis ywrO encodes a nitroreductase enzyme which activates the prodrug $\mathrm{CB}$ 1954. Microbiology 148:297-306.

Aoki, T., S. Egusa and T. Arai. 1975. Reduced nitrofuran sensitivity. Jpn J Microbiol. 19:327-329.

Arai, T., T. Aoki, and S. Egusa. 1975. Mechanisms of decrease of nitrofuran sensitivity conferred by R factors, pp.505-513. In: Mitsuhashi, S., and H. Hashimoto, editors. Microbial Drug Resistance. Baltimore: University Park Press. pp. 505-513. 
Armon, A., D. Graur, and N. Ben-Tal. 2001. ConSurf: An algorithmic tool for the identification of functional regions in proteins by surface-mapping of phylogenetic information. J Mol Biol. 307:447-463.

Asnis, R.E. 1957. The reduction of furacin by cell-free extracts of furacin-resistant and parent-susceptible strains of Escherichia coli. Arch Biochem Biophys. 66:208-216.

Atwell, G.J., M. Boyd, B.D. Palmer, R.F. Anderson, S.M. Pullen, W.R. Wilson, and W.A. Denny. 1996. Synthesis and evaluation of 4-substituted analogues of 5-[N,N-bis(2chloroethyl)amino]-2-nitrobenzamide as bioreductively activated prodrugs using an Escherichia coli nitroreductase. Anti-Cancer Drug Des. 11:553-567.

Bachmann, B.J. 1972. Pedigrees of some mutant strains of Escherichia coli K-12. Bacteriol Rev. 40:116-167.

Bailey, S.M., and I.R. Hart. 1997. Editorial: Nitroreductase activation of CB1954 -- an alternative 'suicide' gene system. Gene Ther. 4:80-81.

Bailey, S.M., R.J. Knox, S.M. Hobbs, T.C. Jenkins, A.B. Mauger, R.G. Melton, P.J. Burke, R.A. Connors, and I.R. Hart. 1996. Investigation of alternative prodrugs for use with Escherichia coli nitroreductase in suicide gene approaches to cancer therapy. Gene Ther. 3:1143-1150.

Bailey, S., A. Lewis, R. Knox, L. Patterson, G. Fisher, and P. Workman. 1998. Reduction of the indoloquinone anticancer drug EO9 by purified DT-diaphorase: a detailed kinetic study and analysis of metabolites. Biochem Pharm. 56:613-621.

Bairoch, A., P. Bucher and K. Hofmann. 1997. The Prosite database, its status in 1997. Nucleic Acids Res. 25:217-221.

Baker, P., K. Britton, D. Rice, A. Rob, and T. Stillman. 1992. Structural consequences of sequence patterns in the fingerprint region of the nucleotide binding fold. Implications for nucleotide specificity. J Mol Biol. 228:662-71.

Barbosa, T.M., and S.B. Levy. 2000. Differential expression of over 60 chromosomal genes in Escherichia coli by constitutive expression of MarA. J Bacteriol. 182:34673474 .

Barker, W., F. Pfeiffer, and D.G. George. 1995. Superfamily and domain. In: Methods in Protein Structure Analysis, M.Z. Atassi and E. Appelaa, editors. New York: Plenum Publishing. pp. 473-481.

Barker, W., F. Pfeiffer, and D.G. George. 1996. Superfamily classification in the PIRInternational Protein Sequence Database. Methods Enzymol. 266:49-71. 
Batemen, A., E. Birney, L. Cerruti, R. Durbin, L. Etwiller, S. Eddy, S. Griffiths-Jones, K. Howe, M. Marshall., and E. Sonnhammer. 2002. The Pfam protein families database. Nucleic Acids Res. 30:276-280.

Bauchinger, M, E. Schmid, F.J. Wiebel, and E. Roscher. 1988. 1,6-Dinitropyrene causes spindle disturbances and chromosomal damage in V79 Chinese hamster cells. Mutat Res. 208:213-218.

Belisario, M.A., R. Pecce, A. Garofalo, N. Sannolo, and A. Malorni. 1996. Erythrocyte enzymes catalyze 1-nitropyrene and 3-nitrofluoranthene nitroreduction. Toxicology 108:101-108.

Bell, C., T. Yeates, and D. Eisenberg. 1997. Unusual conformation of nicotinamide adenine dinucleotide (NAD) bound to diphtheria toxin: A comparison with NAD bound to the oxidoreductase enzymes. Protein Sci. 6:2084-2096.

Bellamacina, C.R. 1996. The nicotinamide dinucleotide binding motif: a comparison of nucleotide binding proteins. FASEB J. 10:1257-1269.

Berman,H.M., J. Westbrook, Z. Feng, G. Gilliland, T.N. Bhat, H. Weissig, I.N.

Shindyalov, P.E. Bourne. 2000. The Protein Data Bank. Nucleic Acids Res. 28:235-242.

Bertenyi, K.K.A., and I.B. Lambert. 1996. The mutational specificity of furazolidone in the lacI gene of Escherichia coli. Mutat Res. 357:199-208.

Bhandari, P., and J. Gowrishankar. 1997. An Escherichia coli host strain useful for efficient overproduction of cloned gene products with $\mathrm{NaCl}$ as the inducer. J Bacteriol. 179:4403-4406.

Biaglow, J.E. 1981. Cellular electron transfer and radical mechanisms for drug metabolism. Radiat Res. 86:212-242.

Birkenbihl, R.P., and W. Vielmetter. 1989. Complete maps of IS1, IS2, IS3, IS4, IS5, IS30 and IS150 locations in Escherichia coli K12. Mol Gen Genet. 220:147-153.

Birkenbihl, R.P., and W. Vielmetter. 1991. Completion of the IS map in E. coli: IS186 positions on the E. coli K-12 chromosome. Mol Gen Genet. 226:318-20.

Blackburn, J. 2000. Engineering and design: Rational versus combinational approaches. Curr Opin Struct Biol. 10:399-400.

Blackwell, J.R., and R. Horgan. 1991. A novel strategy for production of a highly expressed recombinant protein in an active form. FEBS Lett. 295:10-12. 
Blackwood, L., P.J. O'Shaughnessy, S.W. Reid, and D.J. Argyle. 2001. E. coli nitroreductase/CB1954: in vitro studies into a potential system for feline cancer gene therapy. Vet J. 161:269-279.

Blattner F., G. Plunkett, C. Bloch, N. Perna, V. Burland, M. Riley, J. Collado-Vides, J. Glasner, C. Rode, G. Mayhew, J. Gregor, N. Davis, H. Kirkpatrick, M. Goeden, D. Rose, B. Mau, and Y. Shao. The complete genome sequence of Escherichia coli K-12.

Science 277:1453-74.

Blehert, D., B. Fox, and G. Chambliss. 1999. Cloning and sequence analaysis of two Pseudomonas flavoprotein xenobiotic reductases. J Bacteriol. 181:6254-6263.

Boles, E., and T. Miosga. 1995. A rapid and highly efficient method for PCR-based sitedirected mutagenesis using only one new primer. Curr Genet. 28:197-198.

Bolognese F., C. Di Lecce, E. Galli, and P. Barbieri. 1999. Activation and inactivation of Pseudomonas stutzeri methylbenzene catabolism pathways mediated by a transposable element. Appl Environ Microbiol. 65:1876-82.

Bond, J.A., J.L. Mauderly, R.F. Henderson and R.O. McClellan. 1985. Metabolism of 1$\left[{ }^{14} \mathrm{C}\right]$ nitropyrene in respiratory tract tissue of rats exposed to diesel exhaust. Toxicol Appl Pharm. 79:461-470.

Boyd, E.F., and D.L. Hartl. 1997. Nonrandom location of IS1 elements in the genomes of natural isolates of Escherichia coli. Mol Biol Evol. 14:725-732.

Breeze, A.S. and E.E. Obaseiki-Ebor. 1983. Mutations to Nitrofurantoin and Nitrofurazone Resistance in Escherichia coli K12. J Gen Microbiol. 129:99-103.

Bridgewater, J.A., R.J. Knox, J.D. Pitts, M.K. Collins, and C.J. Springer. 1997. The Bystander Effect of the Nitroreductase/CB1954 Enzyme/Prodrug System Is Due to a CellPermeable Metabolite. Hum Gene Ther 8: 709-717.

Bridgewater, J., C. Springer, R. Knox, N. Minton, N. Michael, and M. Collins. 1995. Expression of the bacterial nitroreductase enzyme in mammalian cells renders them selectively sensitive to killing by the prodrug CB1954. Eur J Cancer 31A:2362-70.

Brooks, S.P.J.. 1994. A program for analyzing enzyme rate data obtained from a microplate reader. Biotechniques 17:1154-1161.

Brooks, S.P.J.. 1992. A simple computer program with statistical tests for the analysis of enzyme kinetics. Biotechniques. 13:906-11.

Brosius, J. 1984. Plasmid vectors for the selection of promoters. Gene 27:151-160. 
Bryan, G.T., editor. 1978. Nitrofurans. Raven Press: New York; 248 p.

Bryant, C., and M. DeLuca. 1991. Purification and characterization of an oxygeninsensitive NAD $(\mathrm{P}) \mathrm{H}$ nitroreductase from Enterobacter cloacae. J Biol Chem. 266:4119-4125.

Bryant, C., L. Hubbard, and W.D. McElroy. 1991. Cloning, Nucleotide Sequence, and Expression of the Nitroreductase Gene from Enterobacter cloacae. J Biol Chem. 266: 4126-4130.

Bryant, D.W. and D.R. McCalla. 1980. Nitrofuran induced mutagenesis and error prone repair in Escherichia coli. Chem Biol Interact. 31:151-166.

Bryant, D.W., D.R. McCalla, M. Leeksma, and P. Laneuville. 1981. Type I nitroreductases of Escherichia coli. Can J Microbiol. 27:81-86.

Bryant, D.W., D.R. McCalla, P. Lultschik, M.A. Quilliam, and B.E. McCarry. 1984. Metabolism of 1,8-dinitropyrene by Salmonella typhimurium. Chem Biol Interact. 49:351-368.

Canters, G.W., and C. Dennison. 1995. Biological electron transfer: Structural and mechanistic studies. Biochimie 77:506-515.

Capy P., G. Gasperi, C. Biemont, and C. Bazin. 2000. Stress and transposable elements: co-evolution or useful parasites? Heredity 2000:101-6.

Carroll C.C., D. Warnakulasuriyarachchi, M.R.Nokhbeh, I.B. Lambert. 2002. Salmonella typhimurium mutagenicity tester strains that overexpress oxygen-insensitive nitroreductases $n f s A$ and $n f_{s} B$. Mutat Res. 501:79-98.

Carugo, O., and P. Argos. 1997a. NADP-dependent enzymes. I: Conserved stereochemistry of cofactor binding. Proteins 28:10-28.

Carugo, O., and P. Argos. 1997b. NADP-dependent enzymes. II: Evolution of the monoand dinucleotide binding domains. Proteins 28:29-40.

Cavelier, G., and L. Amzel. 2001. Mechanism of NAD(P)H:quinone reductase: Ab initio studies of reduced flavin. Proteins 2001 43:420-32.

Cedrone, F., A. Ménez, and E. Quéméneur. 2000. Tailoring new enzyme functions by rational redesign. Curr Opin Struct Biol. 10:405-410.

Cerniglia, C.E., J.P. Freeman, G.L. White, R.H. Heflich and D.W. Miller. 1985. Fungal metabolism and detoxification of the nitropolycyclic aromatic hydrocarbon 1-nitropyrene. Appl Environ Microbiol. 50:649-655. 
Chao, L., C. Vargas, B.B. Spear, and E.C. Cox. 1983. Transposable elements as mutator genes in evolution. Nature 303:633-635.

Chatterjee P.K., and N.L. Sternberg. 1995. A general genetic approach in Escherichia coli for determining the mechanism(s) of action of tumoricidal agents: application to DMP 840, a tumoricidal agent. Proc Natl Acad Sci U S A 92:8950-4.

Chen H., A. Lum, A. Seifried, L. Wilkens, and L. Le Marchand. Association of the NAD(P)H:quinone oxidoreductase 609C-->T polymorphism with a decreased lung cancer risk. Cancer Res. 59:3045-8

Chen, R., and S.-S. Jeong. 2000. Functional prediction: Identification of protein orthologs and paralogs. Protein Sci. 9:2344-2353.

Chung-Faye G., D. Palmer, D. Anderson, J. Clark, M. Downes, J. Baddeley, S. Hussain, P. Murray, P. Searle, L. Seymour, P. Harris, D. Ferry, and D. Kerr. 2001. Virus-directed, enzyme prodrug therapy with nitroimidazole reductase: a phase I and pharmacokinetic study of its prodrug, CB1954. Clin Cancer Res. 7:2662-8.

Cui W., B. Gusterson, and A. Clark. 1999. Nitroreductase-mediated cell ablation is very rapid and mediated by a p53-independent apoptotic pathway. Gene Ther. 6:764-70.

Clark, A.J., M. Iwobi, W. Cui, M. Crompton, G. Harold, S. Hobbs, T. Kamalati, R. Knox, C. Neil, F. Yull, and B. Gusterson. 1997. Selective cell ablation in transgenic mice expressing E. coli nitroreductase. Gene Ther. 4:101-110.

Cleland, W.W. 1979. Statistical analysis of enzyme kinetic data. Methods Enzymol. 63:103-38.

Cohen, S.M.. 1978. Toxicity and carcinogenicity of nitrofurans. In: Bryan, G.T., Editor. Nitrofurans. New York: Raven Press. pp. 171-214.

Copeland, R.A. 2000. Enzymes: A practical introduction to structure, mechanism, and data analysis. Wiley-VCH: New York; $397 \mathrm{p}$.

Corbett, M.D., C. Wei and B.R. Corbett. 1985. Nitroreductase-dependent mutagenicity of p-nitrophenylhydroxylamine and its $\mathrm{N}$-acetyl and $\mathrm{N}$-formyl hydroxamic acids. Carcinogenesis 6:727-732.

Corpet, F., F. Servant, J. Gouzy, and D. Kahn. 2000. ProDom and ProDom-CG: tools for protein domain analysis and whole genome comparisons. Nucleic Acids Res 28:267-269. 
Coves, J., M. Zeghouf, D. Macherel, B. Guigliarelli, M. Asso, and M. Fontecave. 1997. Flavin mononucleotide-binding domain of the flavoprotein component of the sulfite reductase from Escherichia coli. Biochemistry 36:5921-5928.

Craig, N. L. 1997. Target site selection in transposition. Annu Rev Biochem. 66:437-474

Creighton, T.E. 1993. Proteins: Structures and Molecular Properties. Second Edition. W.H. Freeman and Company: New York; 507 p.

Cui, W., B. Gusterson, and A. Clark. 2002. Inhibition of myc-dependent breast tumor formation in transgenic mice. Breast Cancer Res Tr. 71:9-20.

Cupples, C.G. and J.H. Miller, 1989. A set of lacZ mutations in Escherichia coli that allow rapid detection of each of the six base substitutions. Proc Natl Acad Sci USA. 86:5345-5349.

Das, A., D. Court, M. Gottesman, and S. Adhya. 1977. Polarity of insertion mutations is caused by Rho-mediated termination of transcription. In: A. I. Bukhari, J. A. Shapiro, and S. L. Adhya, editors, DNA insertion elements, plasmids, and episomes. New York: Cold Spring Harbor Laboratory, Cold Spring Harbor. pp.93-98.

Davies, L.C. 1995. Simple synthesis of the 5-0-benzoylriboside of 1,4-dihydronicotinic acid; A cofactor for DT diaphorase and nitroreductase enzymes. Nucleos Nucleot. 14:311-312.

Dayhoff, M.O., W.C. Barker, and L.T. Hunt. 1983. Establishing homologies in protein sequences. Methods Enzymol. 91:524-45.

Debets-Ossenkopp, Y., R.G.J. Pot, D.J. van Westerloo, A. Goodwin, C. M.J.E. Vandenbrouke-Grauls, D.E. Berg, P.S. Hoffman, and J.G. Kusters. 1999. Insertion of mini-IS605 and deletion of adjacent sequences in the nitroreductase ( $r d x A)$ gene cause metronidazole resistance in Helicobacter pylori NCTC11637. Antimicrob Agents Ch. 43:2657-2662.

Debnath, A., C. Hansch, K. Kim, and Y. Martin. 1993. Mechanistic interpretations of the genotoxicity of nitrofurans (antibacterial agents) using quantitative structure-activity relationships and comparative molecular field analysis. J Med Chem. 36:1007-1016.

DeSerres, F.J. 1974. AF2-food preservative or genetic hazard? Mutat Res. 26:1-2.

DeSerres, F.J., and T. Matsushima. 1986. Mutagenesis and carcinogenesis by nitropyrenes and cancer chemotherapeutics. Mutat Res. 164:3-8. 
DesJarlais, R., R. Sheridan, G. Seibel, J. Dixon, and I. Kuntz. 1988. Using shape complementarity as an initial screen in designing ligands for a receptor binding site of known three-dimensional structure. J Med Chem. 31:722-729.

Djeha, A.H., A. Hulme, M. Dexter, A. Mountain, L. Young, P. Searle, D. Kerr, and C. Wrighton. 2000. Expression of Escherichia coli B nitroreductase in established human tumor zenografts in mice results in potent antitumoral and bystander effects upon systemic administration of the prodrug CB1954. Cancer Gene Ther. 7:721-31.

Djeha, A.H., T. Thomson, H. Leung, P. Searle, L. Young, D. Kerr, P. Harris, A. Mountain, and C. Wrighton. 2001. Combined adnovirus-mediated nitroreductase gene delivery and CB1954 treatment: a well-tolerated therapy for established solid tumours. Mol Ther. 3:233-40.

Djuric, Z., E.K. Fifer, Y. Yamazoe, and F.A. Beland. 1988. DNA binding by 1nitropyrene and 1,6-dinitropyrene in vitro and in vivo: effects of nitroreductase induction. Carcinogenesis 9:357-364.

Djuric, Z., D.W. Potter, R.H. Heflich, and F.A. Beland. 1986. Aerobic and anaerobic reduction of nitrated pyrenes in vitro. Chem Biol Interact. 59:309-324.

Doi, T., H. Yoshimura and K. Tatsumi. 1983. Properties of nitrofuran reductases from Escherichia coli B/r. Chem Pharm Bull. (Tokyo) 31:1105-1107.

Drabek, D., J. Guy, R. Craig and F. Grosveld. 1997. The expression of bacterial nitroreductase in transgenic mice results in specific cell killing by the prodrug CB1954. Gene Ther. 4: 93-100.

Drzyzga, O., A. Schmidt, and K.-H. Blotevogel. 1995. Reduction of nitrated diphenylamine derivatives under anaerobic conditions. Appl Environ Microb. 61:32823287.

Durbin, R., S. Eddy, A. Krogh,, and G. Mitchison. 1998. Biological Sequence Analysis: Probabilistic Models of Proteins and Nucleic Acids: A tutorial introduction to hidden Markov models and other probabilistic modelling approaches in computational sequence analysis. Cambridge University Press, 356 p.

Eddy, E.P., E.C. McCoy, H.S. Rosenkranz and R. Mermelstein. 1986. Dichotomy in the mutagenicity and genotoxicity of nitropyrenes: Apparent effect of the number of electrons involved in nitroreduction. Mutat Res. 161:109-111.

Edmondson, D., and S. Ghisla. 1999. Flavoenzyme structure and function. From:

Methods in Molecular Biology, vol. 131: Flavoprotein Protocols. S.K. Chapman and G.A. Reid, editors. New Jersey: Humana Press Inc.. pp. 157-179. 
Eichenbaum, Z., and Z. Livneh. 1998. UV light induces IS 10 transposition in Escherichia coli. Genetics 149:1173-1181.

Einisto, P., M. Watanabe, M. Ishidate Jr. and T. Nohmi. 1991. Mutagenicity of 30 chemicals in Salmonella typhimurium strains possessing different nitroreductase or $O$ acetyltransferase activities. Mutat Res. 259:95-102.

Elanskaya, I.V., E.A. Chesnavichene, C. Vernotte and C. Astier. 1998. Resistance to nitrophenolic herbicides and metronidazole in the cyanobacterium Synechocystis sp. PCC 6803 as a result of the inactivation of a nitroreductase-like protein encoded by $\operatorname{drg} A$ gene. FEBS Lett. 428:188-192.

Engler, J.A., and M.P. van Bree. 1981. The nucleotide sequence and protein-coding capability of the transposable element IS5. Gene 14:155-163.

Epstein, S.S., S. Joshi, J. Andrea, N. Mantel, E. Sawicki, T. Stanley and E.C. Tabor. 1966. Carcinogenicity of organic particulate pollutants in urban air after administration of trace quantities to neonatal mice. Nature 5068:1305-1307.

Faig, M., M. Bianchet, P. Talalay, S. Chen, S. Winski, D. Ross, and L.M. Amzel. 2000. Structures of recombinant human and mouse $\mathrm{NAD}(\mathrm{P}) \mathrm{H}$ :quinone oxidoreductases: species comparison and structural changes with substrate binding and release. Proc Natl Acad Sci USA 97:3177-3182.

Fifer, E.K., R.H. Heflich, Z. Djuric, P.C. Howard and F.A. Beland. 1986. Synthesis and mutagenicity of 1-nitro-6-nitrosopyrene and 1-nitro-8-nitrosopyrene, potential intermediates in the metabolic activation of 1,6- and 1,8-dinitropyrene. Carcinogenesis 7:65-70.

Fraaije, M., and A. Mattevi. 2000. Flavoenzymes: diverse catalysts with recurrent features. Trends Biochem Sci. 25:126-132.

Franz, R., and H.-G. Neumann. 1986. The reduction of aromatic nitro compounds with $\mathrm{Zn} / \mathrm{Cu}$. A new synthesis of $\mathrm{N}$-acetoxy-N-acetyl-arylamines. Carcinogenesis 7:183-184.

Friedlos, F., S. Court, M. Ford, W. Denny, and C. Springer. 1998. Gene-directed enzyme prodrug therapy: quantitative bystander cytotoxicity and DNA damage induced by CB1954 in cells expressing bacterial nitroreductase. Gene Ther. 5:105-112.

Fromm, H.J. 1975. Initial Rate Enzyme Kinetics. New York: Springer-Verlag. 321 p.

Galas, D. J., and M. Chandler. 1989. Bacterial insertion sequences. In: D. E. Berg, and M. M. Howe, editors. Mobile DNA. American Society for Microbiology, Washington, D.C. p.p.109-162. 
Gamas, P., M.G. Chandler, P. Prentki, and D.J. Galas. 1987. Escherichia coli integration host factor binds specifically to the ends of the insertion sequence IS 1 and to its major insertion hot-spot in pBR322. J Mol Biol. 195:261-272.

Gane, P., and P. Dean. 2000. Recent advances in structure-based rational drug design. Curr Opin Struc Biol. 10:401-404.

Gavin, J.J., F.F. Ebetino, R. Freedman, and W.E. Waterbury. 1966. The aerobic degradation of 1-(5-nitrofurfurylideneamino)-2-imidazolidinone (NF-246) by Escherichia coli. Arch Biochem Biophys. 113:399-404.

Gilcrease, P.C., and V.G. Murphy. 1995. Bioconversion of 2,4-diamino-6-nitrotoluene to a novel metabolite under anoxic and aerobic conditions. Appl Environ Microb. 61:42094214.

Gille C, A. Goede, R. Preissner, K. Rother, C. Frommel. 2000. Conservation of substructures in proteins: interfaces of secondary structural elements in proteasomal subunits. J Mol Biol. 299:1147-54.

Glaser, F., T. Pupko, I.Paz, E. Martz, and N. Ben-Tal. The Consurf Server, an automatic tool for the identification and graphic representation of functional regions in proteins. [manuscript in preparation]

Goodrich, J. A., M. L. Schwartz, and W. R. McClure. 1990. Searching for and predicting the activity of sites for DNA binding proteins: compilation and analysis of the binding sites for Escherichia coli integration host factor (IHF). Nucleic Acids Res. 18:4993-5000

Goodwin, A., D. Kersulyte, G. Sisson, S.J.O. Veldhuyzen van Zanten, D. E. Berg, and P.S. Hoffman. 1998. Metronidazole resistance in Helicobacter pylori is due to null mutations in a gene $(\mathrm{rdxA})$ that encodes an oxygen-insensitive NADPH nitroreductase. Mol Microbiol. 28:383-393.

Gottschall, D.W., and R. Wang. 1995. Depletion and bioavailability of $\left[{ }^{14} \mathrm{C}\right]$ Furazolidone residues in swine tissues. J Agr Food Chem. 43:2520-2525.

Green, N.K., D. Youngs, J. Neoptolemos, F. Friedlos, R. Knox, C. Springer, G. Anlezark, N. Michael, R. Melton, M. Ford, L. Young, D. Kerr, and P. Searle. 1997. Sensitization of colorectal and pancreatic cancer cell lines to the prodrug 5-(aziridin-1-yl)-2,4-

dinitrobenzamide (CB1954) by retroviral transduction and expression of the $E$. coli nitroreductase gene. Cancer Gene Ther. 4:228.

Grove J., P. Searle, S. Weedon, N. Green, I. McNeish, and D. Kerr. 1999. Virus-directed enzyme prodrug therapy using CB1954. Anticancer Drug Des. 14:461-72. 
Grünenfelder, B., G. Rummel, J. vohradsky, D. Röder, H. Langen, and U. Jenal. 2001. Proteomic analysis of the bacterial cell cycle. Proc Natl Acad Sci. USA 98:4681-4686.

Guex, N. and M.C. Peitsch. 1997. SWISS-MODEL and the Swiss-PdbViewer: An environment for comparative protein modeling. Electrophoresis 18:2714-2723.

Hagerman, P. J. 1990. Sequence-directed curvature of DNA. Annu Rev Biochem. 59:755781 .

Hagiwara, Y., M. Watanabe, Y. Oda, T. Sofuni and T. Nohmi. 1993. Specificity and sensitivity of Salmonella typhimurium YG1041 and YG1042 strains possessing elevated levels of both nitroreductase and acetyltransferase activity. Mutat Res. 291:171-180.

Hales, L. M., R. I. Gumport, and J. F. Gardner. 1994. Determining the DNA sequence elements required for binding integration host factor to two different target sites. J. Bacteriol. 176:2999-3006.

Hamilton-Miller, J.M.T. and W. Brumfitt. 1978. Comparative in vitro activity of five nitrofurans. Chemother. 24:161-165.

Hannink, N., S. Rosser, C. French, A. Basran, J. Murray, S. Nicklin, and N. Bruce. 2001. Phytodetoxification of TNT by transgenic plants expressing a bacterial nitroreductase. Nat Biotechnol. 19:1168-1172.

Harada, N., and T. Omura. 1980. Participation of cyctochrome P-450 in the reduction of nitro compounds by rat liver microsomes. J Biochem. (Tokyo) 87:1539-1554.

Hass, B., J. Chen, M. Chou, P. Fu, and R. Heflich. 1987. Binary mixtures containing isomers of nitrobenzo[a]pyrene induce greater-than-additive mutational responses in Salmonella typhimurium. Mutat Res. 190:247-252.

Hasspieler, B., G.D. Haffner, and K. Adeli. 1997. Roles of DT diaphorase in the genotoxicity of nitroaromatic compounds in human and fish cell lines. J Toxicol Env Health 52:137-148.

Hatcher, J.F., K. Yamamoto, M. Ichikawa, G.T. Bryan, and S. Swaminathan. 1995. Metabolic reduction of novel 3,4-dichloro-5-nitrofurans in Salmonella typhimurium. Environ Mol. Mutagen. 25:58-66.

Haynes, C., R. Koder, A.-F. Miller, and D.W. Rodgers. 2002. Structures of nitroreductase in three states: effects of inhibitor binding and reduction. J Biol Chem. 277:11513-20.

Hecht, H. J., C. Bryant, H. Erdmann, H. Pelletier, and R. Sawaya. Crystal structure of nitroreductase from Enterobacter cloacae. To be published. 
Hecht, H.J., H. Erdmann, H.J. Park, M. Sprinzl, and R.D. Schmid. 1995. Crystal structure of NADH oxidase from Thermus thermophilus. Nat Struct Biol. 2:1109- 1114.

Heflich, R.H., Z. Djuric, E.K. Fifer, C.E. Cerniglia and F.A. Beland. 1986. Metabolism of dinitropyrenes to DNA-binding derivatives in vitro and in vivo. In: Carcinogenic and Mutagenic Effects of Diesel Engine Exhaust. Ishinishi, N., Koizumi A., McClellan, R.O., and Stober, W., editors. Elsevier Science Publishers, pp. 185-197.

Heflich, R.H., P.C. Howard and F.A. Beland. 1985. 1-Nitrosopyrene: An intermediate in the metabolic activation of 1-nitropyrene to a mutagen in Salmonella typhimurium TA1538. Mutat Res. 149:25-32.

Hein, D.W. 1988. Acetylator genotype and arylamine-induced carcinogenesis. Biochim Biophys Acta 948: 37-66.

Herreno-Saenz, D., F.E. Evans, F.A. Beland, and P.P. Fu. 1995. Identification of two $\mathrm{N}^{2}$ deoxyguanosinyl DNA adducts upon nitroreduction of the environmental mutagen 1nitropyrene. Chem Res Toxicol. 8:269-277.

Herreno-Saenz, D., F.E. Evans, and P.P. Fui. 1994. Nitroreduction of 1- and 3-nitro7,8,9,10-tetrahydrobenzo[a]pyrene and 1-nitrobenzo[a]pyrene resulting in formation of $N^{2}$-deoxyguanosinyl adducts through long-range migration. Chem Res Toxicol. 7:806814.

Herrlich, P. and M. Schweiger. 1976. Nitrofurans, a group of synthetic antibiotics, with a new mode of action: Discrimination of specific messenger RNA classes. Proc Natl Acad Sci USA 73:3386-3390.

Hetherington, L.H., D.R. Livingstone, and C.H. Walker. 1996. Two-electron and oneelectron dependent in vitro reductive metabolism of nitroaromatics by Mytilus edulis, Carcinus maenas and Asterias rubens. Com Biochem Phys. 113: 231-239.

Higgins, D., and P. M. Sharp. 1989. Fast and sensitive multiple sequence alignments on a microcomputer. CABIOS 5:151-153

Hisamatsu, Y., T. Nishimura, K. Tanabe and H. Matsushita. 1986. Mutagenicity of the photochemical reaction products of pyrene with nitrogen dioxide. Mutat Res. 172:19-27.

Hof, H., T. Chakraborty, R. Royer and J.-P. Buisson. 1987. Mode of action of nitroheterocyclic compounds on Escherichia coli. Drugs Expt Clin Res. XIII:635-639.

Holmes D., and M. Quigley. 1981. A rapid boiling method for the preparation of bacterial plasmids. Anal Biochem. 114:193-7. 
Howard, P.C., and F. Beland. 1982. Xanthine oxidase catalyzed binding of 1-nitropyrene to DNA. Biochem Biophys Res Co. 104:727-732.

Howard, P., T. Flammang, and F. Beland. 1985. Comparison of the in vitro and in vivo hepatic metabolism of the carcinogen 1-nitropyrene. Carcinogenesis 6:243-249.

Howard, P.C., E.C. McCoy and H.S. Rosenkranz. 1987. Sequential and differing nitroreductive pathways for mutagenic nitropyrenes in Salmonella typhimurium. Mutagenesis 2:431-432.

Ingelman, M., S. Ramaswarmy, V. Niviere, M. Fontecave, and H. Eklund. 1999. Crystal structure of NAD(P)H:flavin oxidoreductase from Escherichia coli. Biochemistry 38:7040-7049.

Inouye, S. 1994. NAD(P)H-flavin oxidoreductase from the bioluminescent bacterium, Vibrio fischeri ATCC 7744, is a flavoprotein. FEBS Lett. 347:163-168.

Inouye, S. and H. Nakamura. 1994. Stereospecificity of hydride transfer and substrate specificity for FMN-containing NAD(P)H-flavin oxidoreductase from the luminescent bacterium, Vibrio fischeri ATCC 7744. Biochem Biophys Res Co. 205:275-281.

Jain, R. and N. Forbes. 2001. Can engineered bacteria help control cancer? Proc Natl Acad Sci USA 98:14748-14750.

James A., J. Perry, C. Jay, D. Monget, J. Rasburn, and F. Gould. 2001. Fluorogenic substrates for the detection of microbial nitroreductases. Lett Appl Microbiol 33:403-8.

Jaurin B., and S. Normark. 1983. Insertion of IS 2 creates a novel $a m p C$ promoter in Escherichia coli. Cell 32:809-16.

Jenks, P.J., R. L. Ferrero and A. Labigne. 1999. The role of the $r d x A$ gene in the evolution of metronidazole resistance in Helicobacter pylori. J Antimicrob Chemoth. 43:753-758.

Jenkins, S.T., and P.M. Bennett. 1976. Effect of mutations in deoxyribonucleic acid repair pathways on the sensitivity of Escherichia coli k-12 strains to nitrofurantoin. J Bacteriol. 125:1214-1216.

Jessee, J. 1986. New subcloning efficiency competent cells: $>10^{6}$ transformants $/ \mu \mathrm{g}$. Bethesda Research Laboratory Focus 8:4.

Johnson, J.R., P. Delavari and M. Azar. 1999. Activities of a nitrofurazone-containing urinary catheter and a silver hydrogel catheter against multidrug-resistant bacteria characteristic of catheter-associated urinary tract infection. Antimicrob Agents $\mathrm{Ch}$. 43:2990-2995. 
Jorgensen, M., M. Trend, S. Hazell, and G. Mendz. 2001. Potential involvement of several nitroreductases in metronidazole resistance in Helicobacter pylori. Arch Biochem Biophys. 392:180-191.

Joseph, P., and A. Jaiswal. 1994. NAD(P)H:quinone oxidoreductase 1 (DT-diaphorase) specifically prevents the formation of benzo[a]pyrene quinone-DNA adducts generated by cytochrome P450 and P450 reductase. Proc Natl Acad Sci USA 91:8413-8417.

Josephy, D.P., P. Gruz, and T. Nohmi. 1997. Recent advances in the construction of bacterial genotoxicity assays. Mutat Res. 386:1-23.

Jurado, J., and C. Pueyo. 1995. Role of classical nitroreductase and o-acetyltransferase on the mutagenicity of nifurtimox and eight derivatives in Salmonella typhimurium. Environ Mol Mutagen. 26:86-93.

Kaufman, B.T. 1993. Why NADP? Trends Biochem Sci. 18:278.

Keen, N.T., S. Tamaki, D. Kobayashi, and D. Trollinger. 1988. Improved broad-hostrange plasmids for DNA cloning in Gram-negative bacteria. Gene 70:191-7.

Kidwell, M.G. and D.R. Lisch. 1997. Transposable elements as sources of variation in animals and plants. Proc Natl Acad Sci USA 94:7704-7711.

Kim, K.H., and Y.C. Martin. 1993. Mechanistic interpretation of the genotoxicity of nitrofurans (antibacterial agents) using quantitative structure-activity relationships and comparative molecular field analysis. J Med Chem. 36:1007-1016.

King, C.M. 1988. Metabolism and biological effects of nitropyrene and related compounds. Health Effects Institute. Research Report Number 16.

Kinoshita, K., K. Sadanami, A. Kidera, and N. Go. 1999. Structural motif of phosphatebinding site common to various protein superfamilies: all-against-all structural comparison of protein-mononucleotide complexes. Protein Eng. 12:11-14.

Kinouchi, T., and Y. Ohnishi. 1983. Purification and characterization of 1-nitropyrene nitroreductases from Bacteroides fragilis. Appl Environ Microb. 46:596-604.

Kitts, C., C. Green, R. Otley, M. Alvarez, and P. Unkefer. 2000. Type I nitroreductases in soil enterobacteria reduce TNT (2,4,6-trinitrotoluene) and RDX (hexahydro-1,3,5-trinitro1,3,5-triazine). Can J Microbiol. 46:278-282.

Knox, R.J., F. Friedlos, and M.P. Boland. 1993. The bioactivation of CB 1954 and its use as a prodrug in antibody-directed enzyme prodrug therapy (ADEPT). Cancer Metast Rev. 12:195-212. 
Knox, R.J., F. Friedlos, M. Jarman, L.C. Davies, P. Goddard, G.M. Anlezark, R.G. Melton and R.F. Sherwood. 1995. Virtual cofactors for an Escherichia coli nitroreductase enzyme: Relevance to reductively activated prodrugs in antibody directed enzyme prodrug therapy (ADEPT). Biochem Pharmacol. 49:1641-1647.

Kobierska-Szeliga, M., and H. Czeczot. 1994. Characterization of the genotoxic properties of nitrofurans: nitrofurazone and furazolidone. Acta Biochim Polon. 41:1-5.

Kobori, T., H. Sasaki, W.C. Lee, S. Zenno, K. Saigo, M.E.P. Murphy, and M. Tanokura. 2001. Structure and site-directed mutagenesis of a flavoprotein from Escherichia coli that reduces nitrocompounds. J Biol Chem. 276:2816-2823.

Koder, R.L., and A.-F. Miller. 1998a. Overexpression, isotopic labelling, and spectral characterization of Enterobacter cloacae nitroreductase. Protein Expr Purif. 13:53-60.

Koder, R.L., and A.-F. Miller. 1998b. Steady-state kinetic mechanism, stereospecificity, substrate and inhibitor specificity of Enterobacter cloacae nitroreductase. Biochim Biophys Acta 1387:395-405.

Koder, R., O. Oyedele, and A. Miller. 2001. Retro-nitroreductase, a putative evolutionary precursor to Enterobacter cloacae strain 96-3 nitroreductase. Antioxid Redox Sign. 3:747-55.

Koike, H., H. Sasaki, T. Kobori, S. Zenno, K. Saigo, M.E.P. Murphy, E.T. Adman, and M. Tanokura. 1998. 1.8§ Crystal structure of the major NADH(P)H:FMN oxidoreductase of a bioluminescent bacterium Vibrio fischeri: overall structure, cofactor and substrateanalog binding, and comparison with related flavoproteins. J Mol Biol. 280:259-273.

Koo, H.-S., H.-M. Wu, and D. M. Crothers. 1986. DNA bending at adenine-thymine tracts. Nature 320:501-506

Koziarz, P. 2000. Characterization of NfsA, the major nitroreductase from Escherichia coli [M.Sc. thesis]. Ottawa (ON): Carleton University. 117 p. Available from: Digital Dissertations, UMI Proquest; AAT MZ57773.

Kunst F., N. Ogasawara, I. Moszer, A. Albertini, G. Alloni, V. Azevedo, M. Bertero, P. Bessieres, A. Bolotin, S. Borchert, R. Borriss, L. Boursier, A. Brans, M. Braun, S. Brignell, S. Bron, S. Brouillet, C. Bruschi, B. Caldwell, V. Capuano, N. Carter, S. Choi, J. Codani, I. Connerton, and A. Danchin. 1997. The complete genome sequence of the gram-positive bacterium Bacillus subtilis. Nature 390:249-56.

Kwon, D., F. El-Zaatari, M. Kato, M. Osato, R. Reddy, Y. Yamaoka, and D. Graham. 2000. Analysis of $r d x A$ and involvement of additional genes encoding NAD(P)H flavin 
oxidoreductase (FrxA) and ferredoxin-like protein $(\mathrm{FdxB})$ in metronidazole resistance of Helicobacter pylori. Antimicrob Agents Ch. 44:2133-2142.

Kwon, D., K. Hulten, M. Kato, J. Kim, M. Lee, F. El-Zaatari, M. Osato, and D. Graham. 2001a. DNA sequence analysis of $r d x A$ and frx $A$ from 12 pairs of metronidazole sensitive and -resistant clinical Helicobacter pylori isolates. Antimicrob Agents Ch. 45:26092615.

Kwon, D., M. Lee, J. Kim, F. El-Zaatari, M. Osato, and D. Graham. 2001b. Furazolidoneand nitrofurantoin-resistant Helicobacter pylori: prevalence and role of genes involved in metronidazole resistance. Antimicrob Agents Ch. 45:306-308.

Lakbotia, S.C., A. Sharma, M. Mutsuddi and M.G. Tapadia. 1993. Gelatin as a blocking agent in Southern blot and chromosomal in situ hybridizations. Trends Genet. 9:261.

Lambert, I.B., A.J.E. Gordon, B.W. Glickman and D.R. McCalla. 1992. The influence of local DNA sequence and DNA repair background on the mutational specificity of 1nitroso-8-nitropyrene in Escherichia coli: inferences for mutagenic mechanisms.

Genetics 132:911-927.

Laskowski, R. N. Luscombe, M. Swindells, and J. Thornton. 1996. Protein clefts in molecular recognition and function. Protein Sci. 5:2438-2452.

Latham, J., P. Searle, V. Mautner, and N. James. 2000. Prostate-specific antigen promoter/enhancer driven gene therapy for prostate cancer: Construction and testing of a tissue-specific adenovirus vector. Cancer Res. 60:334-341.

Lee, H., S-H. Cherng, and T-Y. Liu. 1994. Bacterial mutagenicity, metabolism, and dna adduct formation by binary mixtures of benzo[a]pyrene and 1-nitropyrene. Environ Mol Mutagen. 24:229-234.

Lei, B., M. Liu, S. Huang and S.-C. Tu.1994. Vibrio harveyi NADPH-Flavin Oxidoreductase: cloning, sequencing and overexpression of the gene and purification and characterization of the cloned enzyme. J Bacteriol. 176:3552-3558.

Leive, L. 1965. Release of lipopolysaccharide by EDTA treatment of E. coli. Biochem Biophys Res Commun. 21:290-6.

Lemaitre N., W. Sougakoff, C. Truffot-Pernot, and V. Jarlier. 1999. Characterization of new mutations in pyrazinamide-resistant strains of Mycobacterium tuberculosis and identification of conserved regions important for the catalytic activity of the pyrazinamidase PncA. Antimicrob Agents Ch. 43:1761-3. 
Lemmon, M.J., P. van Zij1, M.E. Fox, M.L. Mauchline, A.J. Giaccia, N.P. Minton, and J.M Brown. 1997. Anaerobic bacteria as a gene delivery system that is controlled by the tumor microenvironment. Gene Ther 8:791-6.

Li, A., and J. Dutcher. 1983. Mutagenicity of mono-, di-, and tri- nitropyrenes in Chinese hamster ovary cells. Mutat Res Lett. 119:387-392.

Liang, J., H. Edelsbrunner, and C. Woodward. 1998. Anatomy of protein pockets and cavities: measurement of binding site geometry and implications for ligand design. Protein Sci. 7:1884-1897.

Liang, Q., L. Chen, and A.J. Fulco. 1995. An efficient and optimized PCR method with high fidelity for site-directed mutagenesis. PCR Meth Appl. 4:269-274.

Li, R., M. Bianchet, P. Talalay, and L.M. Amzel. 1995. The three-dimensional structure of $\mathrm{NAD}(\mathrm{P}) \mathrm{H}$ :quinone reductase, a flavoprotein involved in cancer chemoprotection and chemotherapy: Mechanism of the two-electron reduction. Proc Natl Acad Sci USA 92:8846-8850.

Liochev, S.I., A. Hausladen, and I. Fridovich. 1999. Nitroreductase A is regulated as a member of the soxRS regulon of Escherichia coli. Proc Natl Acad Sci USA 96:35373539.

Ling, M., and B.H. Robinson. 1995. A one-step polymerase chain reaction site-directed mutagenesis method for large gene-cassettes with high efficiency, yield and fidelity. Anal Biochem. 230:167-172.

Lipinski, K., A. Djeha, T. Ismail, A. Mountain, L. Young, and C. Wrighton. 2001a. Highlevel, $\beta$-catenin/TCF-dependent transgene expression in secondary colorectal cancer tissue. Mol Ther. 4:365-371.

Lipinski, K., A. Djeha, E. Krausz, D. Lane, P. Searle, A. Mountain, and C. Wrighton. 2001b. Tumour-specific therapeutic adenovirus vectors: repression of transgene expression in healthy cells by endogenous p53. Gene Ther. 8:274-81.

Lovering, A., E. Hyde, P. Searle, and S. White. 2001. The structure of Escherichia coli nitroreductase complexed with nicotinic acid: three crystal forms at $1.7 \AA, 1.8 \AA$ and $2.4 \AA$ resolution. J Mol Biol. In press.

Lu, C., and D.R. McCalla. 1978. Action of some nitrofuran derivatives on glucose metabolism, ATP levels, and macromolecule synthesis in Escherichia coli. Can J Microbiol. 24:650-657.

Lu, C., D.R. McCalla and D.W. Bryant. 1979. Action of nitrofurans on E. coli. Mutation and induction and repair of daughter-strand gaps in DNA. Mutat Res. 67:133-144. 
Makrides, S.C.. 1996. Strategies for achieving high-level expression of genes in Escherichia coli. Microbiol Rev. 60:512-538.

Malia, S.A., and A.K. Basu. 1994. Reductive metabolism of 1-nitropyrene accompanies deamination of cytosine. Chem Res Toxicol. 7:823-828.

Malia, S.A., and A.K. Basu. 1995. Mutagenic specificity of reductively activated 1nitropyrene in Escherichia coli. Biochemistry 34:96-104.

Martin, R.G., W.K. Gillette, S. Rhee, and J.L. Rosner. 1999. Structural requirements for marbox function in transcriptional activation of mar/sox/rob regulon promoters in Escherichia coli: sequence, orientation and spatial relationship to the core promoter. Mol Microbiol. 34:431-441.

Martz, E. 2000. Protein Explorer: Freeware for 3D visualization of macromolecular structure. FASEB J. 14:22.

Mason, R.P. and J.L. Holtzman. 1975. The role of catalytic superoxide formation in the oxygen inhibition of nitroreductase. Biochem Biophys Res Commun. 67:1267-1274.

Matsutani, S. 1997. Genetic analyses of the interactions of the IS1-encoded proteins with the left end of IS1 and its insertion hotspot. J Mol Biol. 267:548-560.

Mattos, C., B. Rasmussen, X.C. Ding, G. Petscko, and D. Ringe. 1994. Analogous inhibitors of elastase do not always bind analogously. Nat Struct Biol. 1:55-58.

Mattos, C., and D. Ringe. 1996. Locating and characterizing binding sites on proteins. Nat Biotechnol. 14:595-599.

Mauger, A.B., P.J. Burke, H.H. Somani, F. Friedlos, and R.J. Knox. 1994. Selfimmolative prodrugs: candidates for antibody-directed enzyme prodrug therapy in conjunction with a nitroreductase enzyme. J Med Chem. 37:3452-3458.

McCalla, D.R. 1979. Nitrofurans. In: Hahn, F., editor. Mechanism of Action of Antibacterial Agents. New York: Springer-Verlag; pp. 176-213.

McCalla, D.R. 1980. Genetic toxicity of some antiprotozoal agents. In: Levandowsky, M., and S. H. Hutner, editors. Biochemistry and Physiology of Protozoa, Second Edition, Vol.3. New York: Academic Press, Inc.. pp.365-384.

McCalla, D.R. 1983. Mutagenicity of nitrofuran derivatives: Review. Environ Mutagen. 5:745-765. 
McCalla, D.R. 1990. Proposed plan for a unified approach for evaluating nitrofuran residues. Drug Metab Rev. 22:863-869.

McCalla, D.R., C. Kaiser, and M.H.L. Green. 1978. Genetics of nitrofurazone resistance in Escherichia coli. J Bacteriol. 133:10-16.

McCalla, D.R., P. Olive and Y. Tu. 1976. Damage to DNA by activated nitrofurans. In: Magee, P., editor. Fundamentals in Cancer Prevention. Baltimore: University Park Press. pp.229-249.

McCalla, D.R., P. Olive, Y. Tu, and M.L. Fan. 1975a. Nitrofurazone-reducing enzymes in E. coli and their role in drug activation in vivo. Can J Microbiol. 21:1485-1491.

McCalla, D.R., A. Reuvers, and C. Kaiser. 1970. Mode of action of nitrofurazone. J Bacteriol. 104:1126-1134.

McCalla, D.R., A. Reuvers, and C. Kaiser. 1971. Breakage of bacterial DNA by nitrofuran derivatives. Cancer Res. 31:2184-2188.

McCalla, D.R., and D. Voutsinos. 1974. On the mutagenicity of nitrofurans. Mutat Res. 26:3-16.

McCalla, D.R., D. Voutsinos and P.L. Olive. 1975b. Mutagen screening with bacteria: Niridazole and nitrofurans. Mutat Res. 31:31-37.

McClintock, B.. 1984. The significance of responses of the genome to challenge. Science 226:792-801.

McCoy, E.C., M. Anders, M. McCartney, P.C. Howard, F.A. Beland and H.S. Rosenkranz. 1984. The recombinogenic inactivity of 1-nitropyrene for yeast is due to a deficiency in a functional nitroreductase. Mutat Res. 139:115-118.

McCoy, E.C., M. Anders and H.S. Rosenkranz. 1983. The basis of the insensitivity of Salmonella typhimurium strain TA98/1,8-DNP 6 to the mutagenic action of nitroarenes. Mutat Res. 121:17-23.

McCoy, E.C., M. Anders, H.S. Rosenkranz and R. Mermelstein. 1985. Mutagenicity of nitropyrenes for Escherichia coli: Requirement for increased cellular permeability. Mutat Res. 142:163-167.

McNeish, I., N. Green, M. Gilligan, M. Ford, V. Mautner, L. Young, D. Kerr, and P. Searle. 1998. Virus directed enzyme prodrug therapy for ovarian and pancreatic cancer using retrovirally delivered $E$. coli nitroreductase and CB1954. Gene Ther. 5:1061-1069. 
McNeish, I.A., P.F. Searle, L.S. Young, and D.J. Kerr. 1997. Gene directed enzyme prodrug therapy for cancer. Adv Drug Deliver Rev. 26:173-184.

McOsker, C.C., and P.M. Fitzpatrick. 1994. Nitrofurantoin: mechanism of action and implications for resistance development in common uropathogens. J Antimicrob Chemoth. 33(suppl. A):23-30.

Mermelstein, R., D.K. Kiriazides, M. Butler, E.C. McCoy and H.S. Rosenkranz. 1981. The extraordinary mutagenicity of nitropyrenes in bacteria. Mutat Res. 89:187-196.

Meyer, J., S. Iida, and W. Arber. 1980. Does the insertion element IS1 transpose preferentially into A+T-rich DNA segments? Molec Gen Genet. 178:471-473.

Michael, N.P., J.K. Brehm, G.M. Anlezark and N.P. Minton. 1994. Physical characterization of the Escherichia coli B gene encoding nitroreductase and its overexpression in Escherichia coli K12. FEMS Microbiol Lett. 124:195-202.

Mittl, P., A. Berry, N. Scrutton, R. Perham, and G. Schulz. 1993. Structural differences between wild-type NADP-dependent glutathione reductase from Escherichia coli and a redesigned NAD-dependent mutant. J Mol Biol. 231:191-5.

Mittl, P., A. Berry, N. Scrutton, R. Perham, and G. Schulz. 1994. Anatomy of an engineered NAD-binding site. Protein Sci. 3:1504-1514.

Möller, L., I. Lax, and L.C. Eriksson. 1993. Nitrated polycyclic aromatic hydrocarbons: a risk assessment for the urban citizen. Environ Health Persp Suppl 101:309-315.

Moreno, S.N.J., R.P. Mason, and R. Docampo. 1984. Reduction of nifurtimox and nitrofurantoin to free radical metabolites by rat liver mitochondria. J Biol Chem. 259:6298-6305.

Morozov, G. I., L. Nosova, S. Biketov, A. Valiaev, and I. Domaradskii. 1994. Biochemical basis for the effect of combining kanamycin and nitrofuran resistance genes in Escherichia coli cells. Mol Gen Mikrobiol. 61:11-14.

Mulrooney, S.B. 1997. Application of a single-plasmid vector for mutagenesis and highlevel expression of thioredoxin reductase and its use to examine flavin cofactor incorporation. Protein Expr Purif. 9:372-378.

Munro, A.W., and M.A. Noble. 1999. Fluorescence analysis of flavoproteins. In: Chapman, S.K., and G.A. Reid, editors. Methods in Molecular Biology, vol. 131: Flavoprotein Protocols. New Jersey: Humana Press Inc. pp. 25-48. 
Murzin A. G., S. Brenner, T. Hubbard, and C. Chothia. 1995. SCOP: a structural classification of proteins database for the investigation of sequences and structures. $\mathrm{J} \mathrm{Mol}$ Biol. 247:536-540.

Naal, Z., J.-H. Park, S. Bernhard, J. Shapleigh, C. Batt, and H. Abruna. 2002. Amperometric TNT biosensor based on the oriented immobilization of a nitroreductase maltose binding protein fusion. Anal Chem. 74:140-148.

Naas, T., M. Blot, W.M. Fitch, and W. Arber. 1994. Insertion sequence-related genetic variation in resting Escherichia coli K-12. Genetics 136:721-730.

Niculescu-Duvaz, I., and C.J. Springer. 1997. Antibody-directed enzyme prodrug therapy (ADEPT): a review. Adv Drug Deliver Rev. 26:151-172.

Niculescu-Duvaz, I., F. Friedlos, D. Niculescu-Duvaz, L. Davies and C. Springer. 1999. Prodrugs for antibody- and gene-directed enzyme prodrug therapies (ADEPT and GDEPT). Anticancer Drug Des. 14:517-538.

Nishida, H., and K. Miki. 1996. Electrostatic properties deduced from refined structures of NADH-cytochrome $b_{5}$ reductase and the other flavin-dependent reductases: pyridine nucleotide-binding and interaction with an electron-transfer partner. Proteins 26:32-41.

Nishihara E., Y. Nagayama, M. Narimatsu, H. Namba, M. Watanabe, M. Niwa, S. Yamashita. 1998. Treatment of thyroid carcinoma cells with four different suicide gene/prodrug combinations in vitro. Anticancer Res. 18:1521-5.

Niviere, V., F. Fieschi, J.-L. Decout, and M. Fontecave. 1996. Is the NAD(P)H:flavin oxidoreductase from Escherichia coli a member of the ferredoxin-NADP ${ }^{+}$reductase family? J Biol Chem. 271:16656-16661.

Novak, M., M.J. Kahley, J. Lin, S.A. Kennedy, and T.G. James. 1995. Involvement of free nitrenium ions, ion pairs, and preassociation trapping in the reactions of ester derivatives of $\mathrm{N}$-arylhydroxylamines and $\mathrm{N}$-arylhydroxamic acids in aqueous solution. $\mathrm{J}$ Org Chem. 60:8294-8304.

Obaseiki-Ebor, E.E. 1984. Enhanced Escherichia coli susceptibility to nitrofurantoin by EDTA and multiple aminoglycoside antibiotics resistance mutation. Chemotherapy. 30:88-91.

Oh B., G. Sarath, P. Shea, R. Drijber, and S. Comfort. 2000. Rapid spectrophotometric determination of 2,4,6-trinitrotoluene in a Pseudomonas enzyme assay. J Microbiol Meth. 42:149-58. 
Orna, M., and R. Mason. 1989. Correlation of kinetic parameters of nitroreductase enzymes with redox properties of nitroaromatic compounds. J Biol Chem. 264:1237912384.

Orr, J.C., D.W. Bryant, D.R. McCalla and M.A. Quilliam. 1985. Dinitropyrene-resistant Salmonella typimurium are deficient in an acetyl-CoA acetyltransferase. Chem Biol Interact. 54:281-288.

Paterson E.S., S.E. Boucher, and I.B. Lambert. 2002. Regulation of the $n f s A$ gene in Escherichia coli by SoxS. J Bacteriol. 184:51-8.

Pattabiraman, N. 2002. Analysis of ligand-macromolecule contacts: computational methods. Curr Med Chem 9:609-21.

Paul, H.E., and M.F. Paul. 1964. The Nitrofurans - Chemotherapeutic Properties. In: Schnitzer, R.J., and F. Hawking, editors. Experimental Chemotherapy, Volume II. New York: Academic Press. pp. 307-369.

Parkinson, G.N., J.V. Skelly, and S. Neidle. 2000. Crystal structure of FMN-dependent nitroreductase from Escherichia coli B: A prodrug-activating enzyme. J Med Chem. 43:3624-3631.

Peterson, F.J., R.P. Mason, J. Hovsepian, and J.L. Holtzman. 1979. Oxygen-sensitive and -insensitive nitroreduction by Escherichia coli and rat hepatic microsomes. J Mol Biol. 254: 4009-4014.

Plapp, B.V. 1995. Site-directed mutagenesis: A tool for studying enzyme catalysis. Methods Enzymol. 249:467-495.

Plesner, I.W. 1986. The apparent $\mathrm{Km}$ is a misleading kinetic indicator. Biochem J. 239:175-178.

Plumb J., A. Bilsland, R. Kakani, J. Zhao, R. Glasspool, R. Knox, T. Evans, and W. Keith. 2001. Telomerase-specific suicide gene therapy vectors expressing bacterial nitroreductase sensitize human cancer cells to the pro-drug CB1954. Oncogene 20:7797803.

Podglajen I., J. Breuil, and E. Collatz. 1994. Insertion of a novel DNA sequence, 1S1186, upstream of the silent carbapenemase gene $c f i A$, promotes expression of carbapenem resistance in clinical isolates of Bacteroides fragilis. Mol Microbiol. 12:105-14.

Podglajen I., J. Breuil, A. Rohaut, C. Monsempes, and E. Collatz. 2001. Multiple mobile promoter regions for the rare carbapenem resistance gene of Bacteroides fragilis. $\mathrm{J}$ Bacteriol. 183:3531-5. 
Pollich, M., and G. Klug. 1995. Identification and sequence analysis of genes involved in late steps in cobalamin (vitamin B12) synthesis in Rhodobacter capsulatus. J Bacteriol. 177: 4481-4487.

Pomposiello, P., and B. Demple. 2000. Identification of SoxS-regulated genes in Salmonella enterica serovar typhimurium. J Bacteriol. 182:23-29.

Prentki, P., M. Chandler, and D.J. Galas. 1987. Escherichia coli integration host factor bends the DNA at the ends of IS1 and in an insertion hotspot with multiple IHF binding sites. EMBO J. 6(8):2479-2487.

Rafii, F., W. Franklin, R. Heflich, and C. Cerniglia. 1991. Reduction of nitroaromatic compounds by anaerobic bacteria isolated from the human gastrointestinal tract. Appl Environ Microb. 57:962-968.

Rafii, F., and E. Hansen. 1998. Isolation of nitrofurantoin-resistant mutants of nitroreductase-producing Clostridium sp. Strains from the human intestinal tract. Antimicrob Agents Ch. 42:1121-1126.

Rafii, F., J.G.H. Ruseler-Van Embden, and Y.F. Asad. 1997. Azoreductase and nitroreductase activity of bacteria in feces from patients with an ileal resevoir. Digest Dis Sci. 42:133-136.

Rafii, F., A.L. Selby, R.K. Newton, and C.E. Cerniglia. 1994. Reduction and mutagenic activation of nitroaromatic compounds by a Mycobacterium sp. Appl Environ Microb. $60: 4263-4267$.

Rafii, R., and C.E. Cerniglia. 1993. Comparison of the azoreductase and nitroreductase from Clostridium perfringens. Appl Environ Microb. 59:1731-1734.

Rasmussen, B.A., K. Bush, and F.P. Tally. 1997. Antimicrobial resistance in anaerobes. Clin Infect Dis. 24:S110-20.

Rescigno, M., and R.N. Perham. 1994. Structure of the NADPH-binding motif of glutathione reductase: efficiency determined by evolution. Biochemistry 33:5721-27.

Rieble, S., D.K. Joshi and M.H. Gold. 1994. Aromatic nitroreductase from the basidiomycete Phanerochaete chrysosporium. Biochem Biophys Res Co. 205: 298-304.

Riley, M., and M. Serres. 2000. Interim report on genomics of Escherichia coli. Annu Rev Microbiol. 54:341-411.

Rodriguez, R.L., and R.C. Tait. 1983. Recombinant DNA Techniques: An Introduction. Menlo Park, CA: Benjamin/Cummings Publishing Company, Inc. 236p. 
Rosenkranz, H.S., E.C. McCoy, R. Mermelstein, and W.T. Speck. 1981. A cautionary note on the use of nitroreductase-deficient strains of Salmonella typhimurium for the detection of nitroarenes as mutagens in complex mixtures including diesel exhausts. Mutat Res. 91:103-105.

Rosenkranz, E.J., E.C. McCoy, R. Mermelstein and H.S. Rosenkranz. 1982. Evidence for the existence of distinct nitroreductases in Salmonella typhimurium: roles in mutagenesis. Carcinogenesis 3:121-123.

Rosenkranz, H.S., E.C. McCoy, D.R. Sanders, M. Butler, D.K. Kiriazides and R. Mermelstein. 1980. Nitropyrenes: Isolation, identification, and reduction of mutagenic impurities in carbon black and toners. Science 209:1039-1042.

Rosenkranz, H.S., and R. Mermelstein. 1983. Mutagenicity and genotoxicity of nitroarenes: All nitro-containing chemicals were not created equal. Mutat Res. 114:217267.

Rosenkranz, H.S., and R. Mermelstein. 1985. The genotoxicity, metabolism and carcinogenicity of nitrated polycyclic hydrocarbons. J Environ Sci Heal. 2:221-272.

Rost, B., R. Schneider, and C. Sander. 1997. Protein fold recognition by prediction-based threading. J Mol Biol 270:1-10.

Rozenski, J., C.J. De Ranter and H. Verplanken.1995. Quantitative structure-activity relationships for antimicrobial nitroheterocyclic drugs. Quant Struct-Act Rel. 14:134141.

Saito, K., A. Shinohara, T. Kamataki, and R. Kato. 1985. Metabolic activation of mutagenic N-hydroxylarylamines by O-acetyltransferase in Salmonella typhimurium TA98. Arch Biochem Biophys. 239:286-295.

Saito, K., A. Shinohara, T. Kamataki, and R. Kato. 1986. A new assay for Nhydroxyarylamine O-acetyltransferase: reduction of n-hydroxyarylamines through $\mathrm{N}$ acetoxyarylamines. Anal Biochem. 152:226-231.

Salmeen, I., A.M. Durisin, T.J. Prater, T. Riley and D. Schuetzle. 1982. Contribution of 1 -nitropyrene to direct-acting Ames assay mutagenicities of diesel particulate extracts. Mutat Res. 104:17-23.

Sambrook, J., E. Fritsch, and T. Maniatis. 1989. Molecular Cloning: A Laboratory Manual, Second Edition, v.1-3. New York: Cold Spring Harbor Laboratory Press.

Sastry, S.S., and R. Jayaraman. 1984. Nitrofurantoin-resistant mutants of Escherichia coli: Isolation and mapping. Mol Gen Genet. 196:379-380. 
Scheepers, P.T.J., M.M.E. Straetemans, J.P. Koopman, and R.P. Bos. 1994. Nitroreduction and formation of hemoglobin adducts in rats with a human intestinal microflora. Environ Health Persp 102(suppl 6):39-41.

Scheibner, K., M. Hofrichter, A. Herre, J. Michels, and W. Fritsche. 1997. Screening for fungi intensively mineralizing 2,4,6-trinitrotoluene. Appl Microbiol Biotechnol. 47:452457.

Schein, C.H. 1989. Production of soluble recombinant proteins in bacteria. Bio/technology 7:1141-1148.

Schenzle, A., H. Lenke, J. Spain, and H.-J. Knackmuss. 1999. Chemoselective nitro group reduction and reductive dechlorination initiate degradation of 2-chloro-5nitrophenol by Ralstonia eutropha JMP134. Appl Environ Microb. 65:2317-2323.

Scrutton, N., A. Berry, and R. Perham. 1990. Redesign of the coenzyme specificity of a dehydrogenase by protein engineering. Nature 343:38-43.

Shindyalov I.N., P.E. Bourne. 1998. Protein structure alignment by incremental combinatorial extension (CE) of the optimal path. Protein Eng 11:739-747.

Searle, P., S. Weedon, I. McNeish, M. Gilligan, M. Ford, F. Friedlos, C. Springer, L. Young, and D. Kerr. 1998. Sensitization of human ovarian cells to killing by the prodrug CB1954 following retroviral or adenoviral transfer of the E. coli nitroreductase gene. Adv Exp Med Biol. 451:107-113.

Segel, I. 1975. Enzyme Kinetics. New York: John Wiley and Sons; 957p.

Sherwood, R.F. 1996. Advanced drug delivery reviews: enzyme prodrug therapy. Adv Drug Deliver Rev. 22:269-288.

Shibata, T, A. Giaccia, and J. Brown. 2002. Hypoxia-inducible regulation of a prodrugactivating enzyme for tumor-specific gene therapy. Neoplasia 4:40-8.

Shuker, S.B., P. Hajduk, R. Meadows, and S. Fesik. 1996. Discovering high-affinity ligands for proteins: SAR by NMR. Science 274:1531.1534.

Siim, B.B., G.J. Atwell, R.F. Anderson, P. Wardman, S.M. Pullen, W.R. Wilson, and W.A. Denny. 1997. Hypoxia-selective antitumor agents. 15. Modification of rate of nitroreduction and extent of lysosomal uptake by polysubstitution of 4-(alkylamino)-5nitroquinoline bioreductive drugs. J Med Chem. 40:1381-1390.

Siim, B.G., and W.R. Wilson. 1995. Efficient redox cycling of nitroquinoline bioreductive drugs due to aerobic nitroreduction in Chinese hamster cells. Biochem Pharm. 50:75-82. 
Sisson, G., J. Jeong, A. Goodwin, L. Bryden, N. Rossler, S. Lim-Morrison, A. Raudonikience, D. Berg, and P. Hoffman. 2000. Metronidazole activation is mutagenic and causes DNA fragmentation in Helicobacter pylori and in Escherichia coli containing a cloned $H$. pylori $r d x A^{+}$. J Bacteriol. 182:5091-5096.

Skelly, J.V., P.J. Collins, R.J. Knox, G.M. Anlezark and R.G. Melton. 1994. Crystallization and preliminary crystallographic data for an FMN-dependent nitroreductase from Escherichia coli B. J Mol Biol. 238:852-853.

Sobolev, V., A. Sorokine, J. Prilusky, E. Abola, and M. Edelman. 1999. Automated analysis of interatomic contacts in proteins. Bioinformatics 15:327-332.

Solovyev, V.V., and N.A. Kolchanov. 1994. Search for functional sites using consensus sequences. In: Kolchanov, N., and H. Lim, editors. Computer analysis of Genetic macromolecules. New Jersey: World Scientific. pp.16-21.

Somerville, C.C., S.F. Nishinio, and J.C. Spain. 1995. Purification and characterization of nitrobenzene nitroreductase from Pseudomonas pseudoalcaligenes JS45. J Bacteriol. 177:3837-3842.

Sotriffer, C., and G. Klebe. 2002. Identification and mapping of small-molecule binding sites in proteins: computational tools for structure-based drug design. Farmaco 57:243-51.

Spain, J.C. 1995. Biodegradation of nitroaromatic compounds. Annu Rev Microbiol. 49:523-555.

Spooner, R.A., K.A. Maycroft, H. Paterson, F. Friedlos, C.J. Springer, and R. Marais. 2001. Appropriate subcellular localisation of prodrug-activating enzymes has important consequences for suicide gene therapy. Int J Cancer 93:123-30.

Stuber, D., H. Matile, and G. Garotta. 1990. System for high-level production in Escherichia coli and rapid purification of recombinant proteins: application to epitope mapping, preparation of antibodies, and structure-function analysis. Immun Meth. 4:121152.

Studier, F.W., and B.A. Moffatt. 1986. Use of bacteriophage T7 RNA polymerase to direct selective high-level expression of cloned genes. J Mol Biol.189:113-130.

Szybalski, W., and V. Bryson. 1952. Genetic studies on microbial cross resistance to toxic agents. J. Bacteriol. 64:489-499.

Tankovic, J., D. Lamarque, J.-C. Delchier, C.-J. Soussy, A. Labigne, and P.J. Jenks. 2000. Frequent association between alteration of the $r d x A$ gene and metronidazole 
resistance in French and North African isolates of Helicobacter pylori. Antimicrob Agents Ch. 44:608-613.

Tanner, J.J., B. Lei, S.-C. Tu, and K.L. Krause. 1996. Flavin Reductase P: Structure of a dimeric enzyme that reduces flavin. Biochemistry 35:13531-13539.

Tanner, J. J., S.-C. Tu, L. Barbour, C. Barnes, and K. Krause. 1999. Unusual folded conformation of nicotinamide adenine dinucleotide bound to flavin reductase P. Protein Sci. 8:1725.

Tatsumi, K., S. Kitamura and N. Narai. 1986. Reductive metabolism of aromatic nitro compounds including carcinogens by rabbit liver preparations. Cancer Res. 46:10891093.

Tazima, Y., T. Kada, and A. Murakami. 1975. Mutagenicity of nitrofuran derivatives, including furylfuramide, a food preservative. Mutat Res. 32:55-80.

Tindall, K.R., and T.A. Kunkel. 1988. Fidelity of DNA synthesis by the Thermus aquaticus DNA polymerase. Biochemistry 27:6008-6013.

Tocher, J.H. 1997. Reductive activation of nitroheterocyclic compounds. Gen Pharmacol. 28:485-487.

Tokiwa, H., R. Nakagawa, and Y. Ohnishi. 1981. Mutagenic assay of aromatic nitro compounds with Salmonella typhimurium. Mutat Res. 91:321-325.

Tokiwa, H., and Y. Ohnishi. 1986. Mutagenicity and carcinogenicity of nitroarenes and their sources in the environment. CRC Crit Rev Toxicol. 17:23-60.

Townson, S.M., P.F.L. Boreham, P. Upcroft, and J.A. Upcroft. 1994. Resistance to nitroheterocyclic drugs. Acta Trop. 56:173-194.

Tu, S.-C., and H.I.X. Mager. 1995. Biochemistry of bacterial luminescence. Photochem Photobiol. 62:615-624.

Tu, Y., and D.R. McCalla. 1975. Effect of activated nitrofurans on DNA. Biochim Biophys Acta 402:142-149.

Ueda, H., M. Nakanishi, E. Suzuki, and T. Nagamune. 1995. Enhancement of mutation frequency with nucleotide triphosphate analogs in pcr random mutagenesis. J Ferment Bioeng. 79:303-305.

Ulanovsky, L.E., and E.N. Trifonov. 1987. Estimation of wedge components in curved DNA. Nature 326:720-722. 
Upcroft, P., and J. Upcroft. 2001. Drug targets and mechanisms of resistance in the anaerobic protozoa. Clin Microbiol Rev. 14:150-164.

Vasudevan, S., D. Shaw, and W. Armarego. 1988. Dihydropteridine reductase from Escherichia coli. Biochem J. 255:581-588.

Vieira, J., and J. Messing. 1982. The pUC plasmids, an M13mp7-derived system for insertion mutagenesis and sequencing with synthetic universal primers. Gene 34:87-93.

Vroomen, L.H.M., M.C.J. Berghmans, J.P. Groten, J.H. Koeman and P.J. van Bladeren. 1988. Reversible interaction of a reactive intermediate derived from furazolidone with glutathione and protein. Toxicol Appl Pharm. 95:53-60.

Wagner, E., M. Schweiger, H. Ponta, and P. Herrlich. 1977. Messenger-selective inhibitor for the initiation of translation in Escherichia coli: Nitrofurantoin. FEBS Lett. 83:337340 .

Wang, C., B. Behrens, M. Ichikawa, and G. Bryan. 1974. Nitroreduction of 5-nitrofuran derivatives by rat liver xanthine oxidase and reduced nicotinamide adenine dinucleotide phosphate-cytochrome c reductase. Biochem Pharmacol. 23:3395-3404.

Wang, H., B. Lei, and S.-C. Tu. 2000. Vibrio harveyi NADPH-FMN oxidoreductase: Arg203 as a critical residue for NADPH recognition and binding. Biochemistry 39:78137819.

Watanabe, M. 2001. Can bioremediation bounce back? Nat Biotech. 19:1111-1115.

Watanabe, M., M. Ishidate Jr. and T. Nohmi. 1990a. Nucleotide sequence of Salmonella typhimurium nitroreductase gene. Nucleic Acids Res. 18:1059.

Watanabe, M., M. Ishidate Jr. and T. Nohmi. 1990b. Sensitive method for the detection of mutagenic nitroarenes and aromatic amines: new derivatives of Salmonella typhimurium tester strains possessing elevated O-acetyltransferase levels. Mutat Res. 234:337-348.

Watanabe, M., M. Ishidate Jr. and T. Nohmi. 1989. A sensitive method for the detection of mutagenic nitroarenes: construction of nitroreductase-overproducing derivatives of Salmonella typhimurium strains TA98 and TA100. Mutat Res. 216:211-220.

Watanabe, M., T. Nishino, K. Takio, T. Sofuni, and T. Nohmi. 1998. Purification and characterization of wild-type and mutant "classical" nitroreductases of Salmonella typhimurium. J Biol Chem. 273:23922-23928. 
Watanabe, M., T. Nohmi, and M. Ishidate, Jr. 1987. New tester strains of Salmonella typhimurium highly sensitive to mutagenic nitroarenes. Biochem Biophys Res Co. 147:974-979.

Watanabe, M., T. Sofuni and T. Nohmi. 1992. Involvement of $\mathrm{Cys}^{69}$ residue in the catalytic mechanism of $N$-hydroxyarylamine $O$-acetyltransferase of Salmonella typhimurium. J Biol Chem. 267:8429-8436.

Weedon, S., N. Green, I. McNeish, M. Gilligan, V. Mautner, C. Wrighton, A. Mountain, L. Young, D. Kerr, and P. Searle. 2000. Sensitisation of human carcinoma cells to the prodrug CB1954 by adenovirus vector-mediated expression of $E$. coli nitroreductase. Int $\mathrm{J}$ Cancer 86:848-854.

Wentzell, B., and D.R. McCalla. 1980. Formation and excision of nitrofuran-DNA adducts in Escherichia coli. Chem Biol Interact. 31:133-150.

Westphal, E., J. Ge, J. Catchpole, M. Ford, and S. Kenney. 2000. The nitroreductase/CB1954 combination in Epstein-Barr virus-positive B-cell lines: induction of bystander killing in vitro and in vivo. Cancer Gene Th. 7:97-106.

Wheeler D.L., C. Chappey, A.E. Lash, D.D. Leipe, T.L. Madden, G.D. Schuler, T.A. Tatusova, and B.A. Rapp. 2000. Database resources of the National Center for Biotechnology Information. Nucleic Acids Res 28:10-4.

Whiteway, J., P. Koziarz, J. Veall, N. Sandhu, P. Kumar, B. Hoecher, and I.B. Lambert. 1998. Oxygen-insensitive nitroreductases: analysis of the roles of $n f s A$ and $n f s B$ in development of resistance to 5-nitrofuran derivatives in Escherichia coli. J Bacteriol. 180:5529-5539.

Wierenga, R.K., M.C.H. De Maeyer, and W.G.J. Hol. 1985. Interaction of pyrophosphate moieties with $\alpha$-helixes in dinucleotide binding proteins. Biochemistry 24:1346-1357.

Wilson, W., S. Pullen, A. Hogg, N. Helsby, K. Hicks, and W. Denny. 2002. Quantitation of bystander effects in nitroreductase suicide gene therapy using three-dimensional cell cultures. Cancer Res. 62:1425-1432.

Wolpert, M., J. Althaus, and D. Johns. 1973. Nitroreductase activity of mammalian liver aldehyde oxidase. J Pharmacol Exp Ther. 185:202-213.

Yabannavar, A.V., and G.J. Zylstra. 1995. Cloning and characterization of the genes for p-nitrobenzoate degradation from Pseudomonas picketti YH105. Appl Environ Microb 61:4284-4290. 
Yamada, M., J.J. Espinosa-Aguirre, M. Watanabe, K. Matsui, T. Sofuni and T. Nohmi. 1997. Targeted disruption of the gene encoding the classical nitroreductase enzyme in Salmonella typhimurium Ames test strains TA1535 and TA1538. Mutat Res. 375:9-17.

Yelamos, B., E. Nunez, J. Gomez-Gutierrez, M. Datta, B. Pacheco, D.L. Peterson, and F. Gavilanes. 1999. Circular dichroism and fluorescence spectroscopic properties of the major core protein of feline immunodeficiency virus and its tryptophan mutants. Eur $\mathrm{J}$ Biochem. 266:1081-1089.

Zablotowicz, R.M., M. A. Locke, and R.E. Hoagland. 1997. Aromatic nitroreduction of acifluorgen in soils, rhizospheres, and pure cultures of rhizobacteria. In: Kruger, E., T. Anderson, and J. Coats, editors. Phytoremediation of Soil and Water Contaminants. Washington, DC: American Chemical Society. pp38-53.

Zenno, S., R. Kobori, M. Tanokura, and K. Saigo. 1998a. Conversion of NfsA, the major Escherichia coli nitroreductase, to a flavin reductase with an activity similar to that of Frp, a flavin reductase in Vibrio harveyi, by a single amino acid substitution. J Bacteriol. 180:422-425.

Zenno, S., R. Kobori, M. Tanokura, and K. Saigo. 1998b. Purification and characterization of NfrA1, a Bacillus subtilis nitro/flavin reductase capable of interacting with the bacterial luciferase. Biosci Biotech Bioch. 62:1978-1987.

Zenno, S., H. Koike, A.N. Kumar, R. Jayaraman, M. Tanokura and K. Saigo. 1996a. Biochemical characterization of NfsA, the Escherichia coli major nitroreductase exhibiting a high amino acid sequence homology to FRP, a Vibrio harveyi flavin oxidoreductase. J Bacteriol. 178:4508-4514.

Zenno, S., H. Koike, M. Tanokura, and K. Saigo. 1996b. Gene cloning, purification, and characterization of NfsB, a minor oxygen-insensitive nitroreductase from Escherichia coli, similar in biochemical properties to FRase I, the major flavin reductase in Vibrio fischeri. J Biochem. 120:736-744.

Zenno, S., H. Koike, M. Tanokura, and K. Saigo. 1996c. Conversion of NfsB, a minor Escherichia coli nitroreductase, to a flavin reductase similar in biochemical properties to FRase I, the major flavin reductase in Vibrio fischeri, by a single amino acid substitution. J Bacteriol. 178:4731-4733.

Zenno, S., and K. Saigo. 1994. Identification of the genes encoding NAD(P)H-flavin oxidoreductases that are similar in sequence to Escherichia coli Fre in four species of luminous bacteria: Photorhabdus luminescens, Vibrio fischeri, Vibrio harveyi, and Vibrio orientalis. J Bacteriol. 17:3544-3551. 
Zenno, S., K. Saigo, H. Kanoh, and S. Inouye. 1994. Identification of the gene encoding the major NAD(P)H-flavin oxidoreductase of the bioluminescent bacterium Vibrio fischeri ATCC 7744. J Bacteriol. 176:3536-3543.

Zerbib, D., P. Gamas, M. Chandler, P. Prentki, S. Bass, and D. Galas. 1985. Specificity of insertion of IS1. J Mol Biol. 185:517-524. 